\title{
The Effect of Heat Transfer on Turbine Performance
}

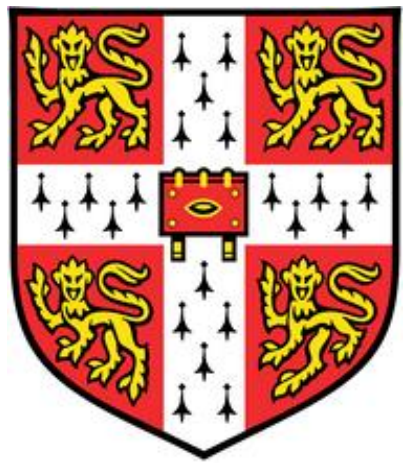

\section{Lachlan James Jardine}

Department of Engineering

University of Cambridge

This dissertation is submitted for the degree of

Doctor of Philosophy 



\section{Declaration}

This thesis is the result of my own work and includes nothing which is the outcome of work done in collaboration except as declared in the Preface and specified in the text. It is not substantially the same as any that I have submitted, or, is being concurrently submitted for a degree or diploma or other qualification at the University of Cambridge or any other University or similar institution except as declared in the Preface and specified in the text. I further state that no substantial part of my thesis has already been submitted, or, is being concurrently submitted for any such degree, diploma or other qualification at the University of Cambridge or any other University or similar institution except as declared in the Preface and specified in the text. It does not exceed the prescribed word limit for the relevant Degree Committee. This dissertation contains less than 65,000 words including appendices, bibliography, footnotes, tables and equations and has fewer than 150 figures.

Lachlan James Jardine

December, 2019 



\title{
The Effect of Heat Transfer on Turbine Performance
}

\author{
Lachlan James Jardine
}

\begin{abstract}
Despite the blades downstream of the combustor being cooled in most large gas turbines, the effect this cooling has on their performance is still unclear. Fundamental questions, such as how turbine efficiency should be defined and which flow features reduce performance, remain unanswered. This thesis answers these questions and addresses the problem of how heat transfer affects turbine performance.

Currently, there is a direct contradiction between industrial experience and the academic methods used to evaluate turbine performance. In a cooled turbine, these entropycentric (exergy) methods predict that the irreversible heat transfer due to cooling would cause an extremely large drop in turbine efficiency (around 4-6\%). In practice, this drop is not observed.

By applying a new method called euergy, this thesis demonstrates that the performance of a cooled turbine can be defined in a way that agrees with industrial experience. This method shares the view of the practical device that the ideal work is defined by a reversible adiabatic turbine. A key consequence of this method is the value placed on heat, relative to work, becomes set by the Joule (Brayton) cycle efficiency. This means whenever heat is transferred, or when viscous reheat occurs, the value of this heat should be set by the Joule cycle efficiency.

This new understanding finally allows both the efficiency to be defined and the flow features that change it to be identified. The method also provides cooling designers with a new way of raising turbine efficiency, a form of thermal recuperation in the flow. This mechanism offers the exciting potential that future cooling systems, when added to a blade profile, could reduce profile loss by up to $9 \%$.

Furthermore, the generality of the new method allows all cooled components, however complex, to be systematically analysed for the first time. This is demonstrated using a computational approach. The large-scale effect of heat transfer on performance is captured in
\end{abstract}


newly developed loss models which are then compared with conjugate heat transfer simulations. The small-scale effect of heat transfer on performance is investigated by examining the near-wall region of cooled boundary layers using direct numerical simulation (DNS).

This thesis establishes a new framework and illustrates how heat transfer affects turbine performance. This new understanding allows performance to be communicated across all levels of turbine design. The new method offers the prospect of future innovative cooling schemes which, in addition to cooling the blade, act to raise turbine efficiency. 


\section{Acknowledgements}

The author is grateful to all the organisations and individuals who have helped support this work. Chief among them is Rob Miller. His guidance and support have enabled this project to impact both academic research and industrial practice.

The financial support provided by the Engineering and Physical Sciences Research Council (EPSRC) and Rolls-Royce plc. has been greatly appreciated. The author is thankful for the computational support from Cambridge Service for Data Driven Discovery (CSD3) and Academic Research Computing High End Resource (ARCHER). In addition, the author is thankful for the travel awards received from King's College, Cambridge, and the American Society of Mechanical Engineers.

From Rolls-Royce, the time and counsel provided by Jon Ebacher, Paolo Adami, Geoff Dailey, Mark Stokes and Fred Goenaga has been invaluable.

From the Whittle Laboratory, the author is particularly grateful to Andrew Wheeler, Nick Atkins, Graham Pullan and Chez Hall for their assistance. Thanks also to Chris Clark for providing the turbine blade profile used throughout this thesis.

From the Osney Laboratory, Budimir Rosic has been exceptionally supportive. Throughout the project, his patience, wisdom and supervision has been greatly valued.

The author was lucky enough to share their $\mathrm{PhD}$ journey with many brilliant people, including Masha Folk. Her enthusiasm was a light, when all other lights went out.

For everything else, I thank my family and Sophie Harrington. They have been constantly supportive and always understanding. 



\section{Contents}

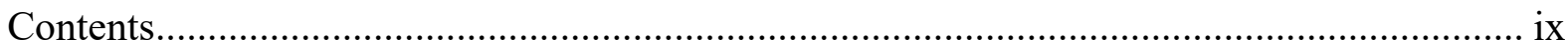

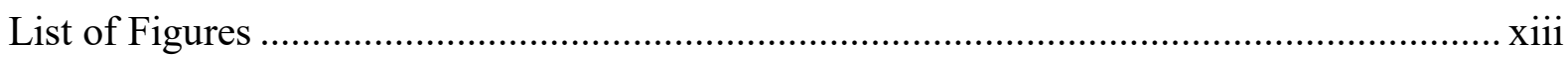

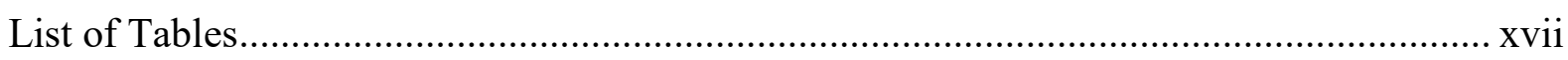

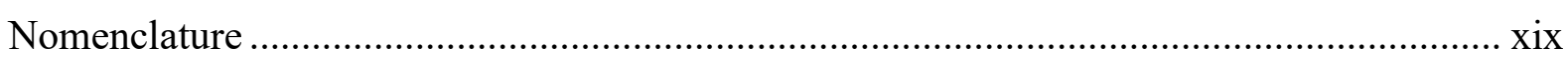

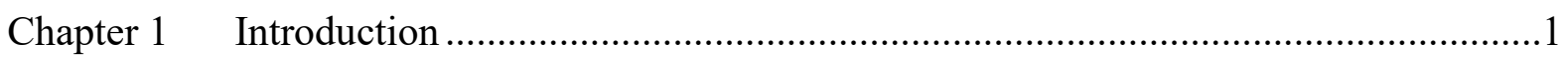

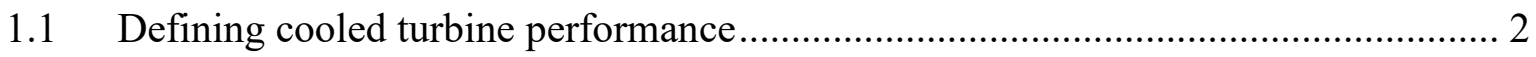

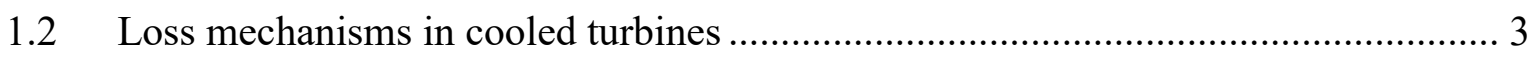

1.3 Practical measurement of cooled turbine performance .......................................... 4

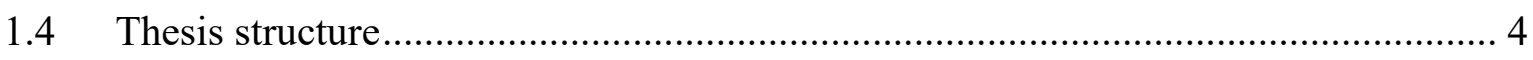

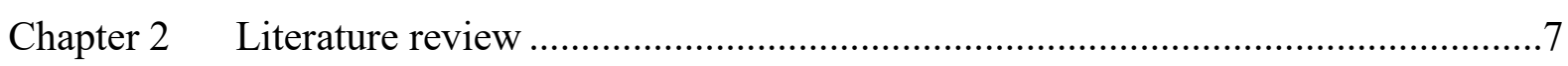

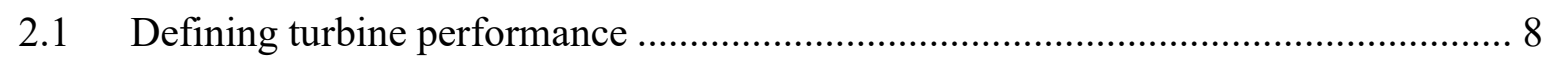

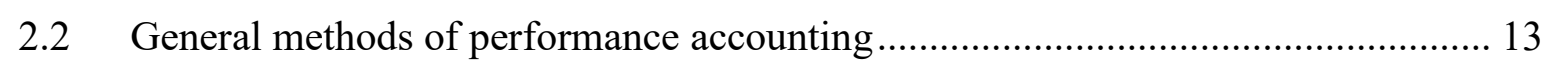

2.3 Modelling loss mechanisms in turbomachinery .................................................. 15

2.4 Effect of heat transfer on boundary layer flow ................................................... 17

2.5 Practical trade-offs in cooled turbine blade design ............................................ 20

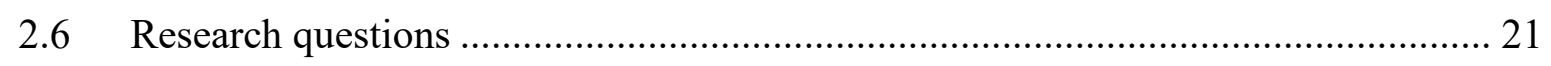

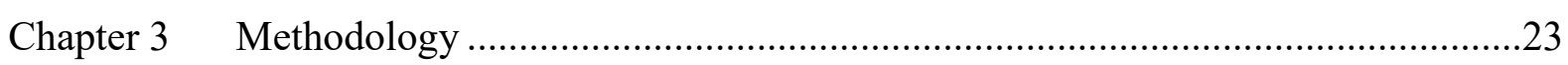

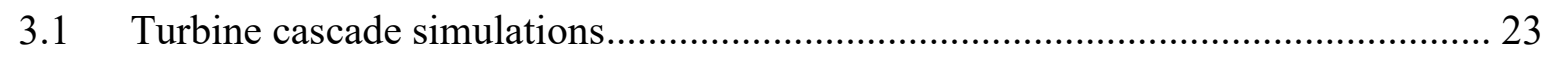

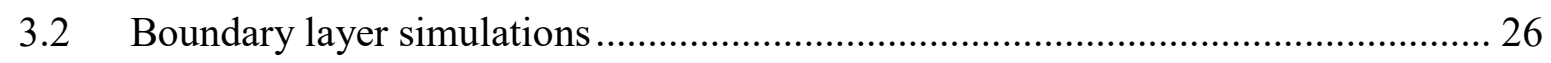

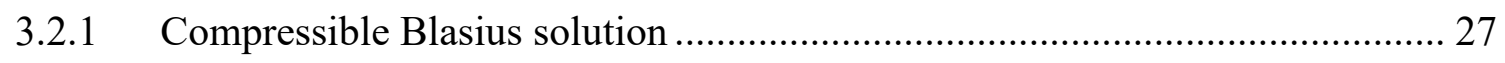

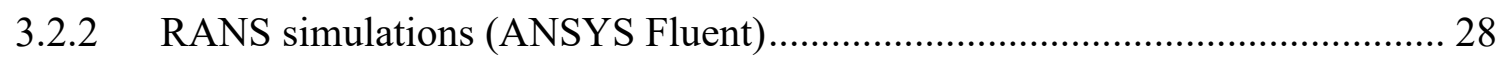

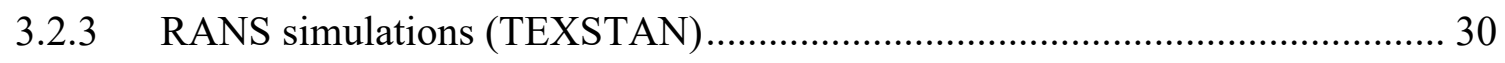

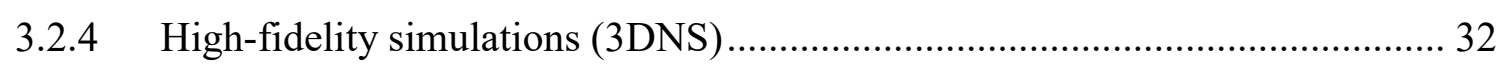

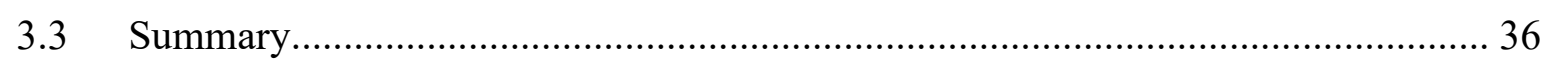

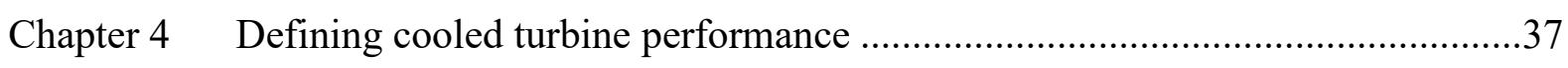

4.1 Comparing general methods (exergy and euergy)............................................ 38

4.2 Comparing industrially-focussed methods (euergy and power-balance) ................ 44

4.3 Extending adiabatic loss coefficients to cooled turbines....................................... 49 


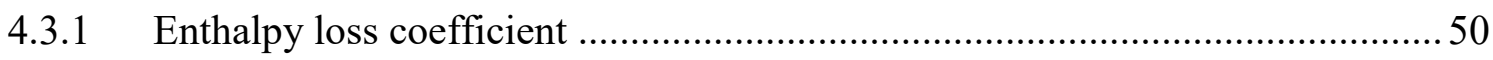

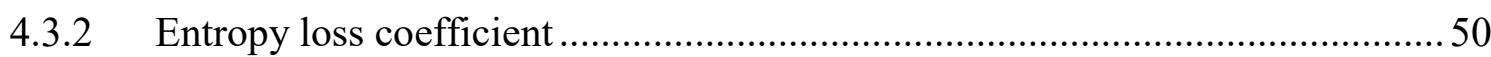

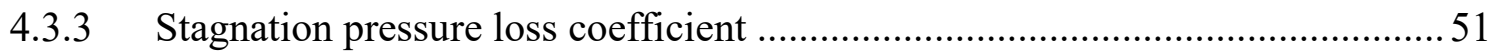

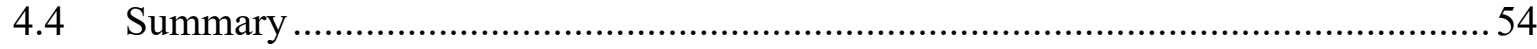

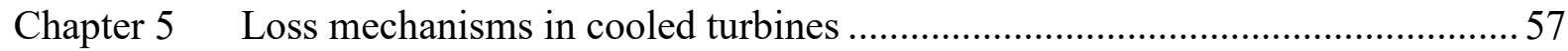

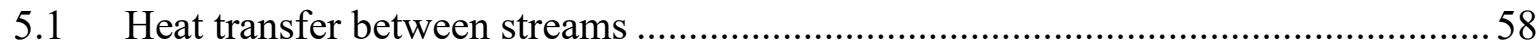

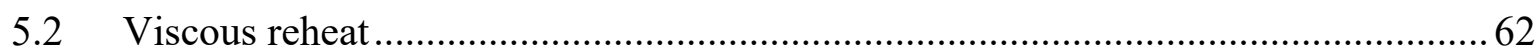

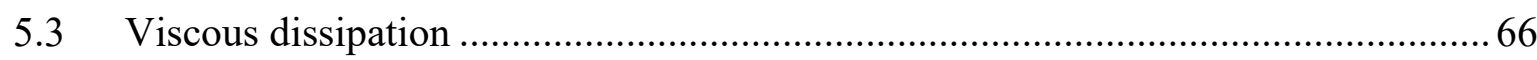

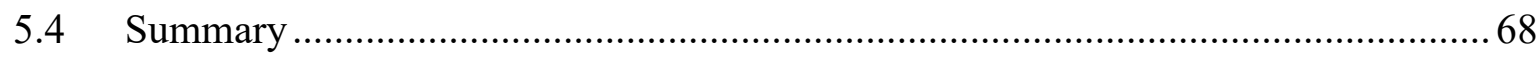

Chapter 6 Modelling loss mechanisms in cooled turbines ............................................. 71

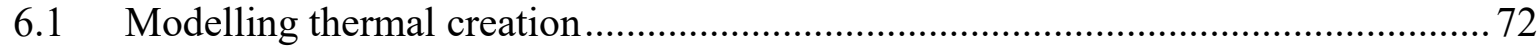

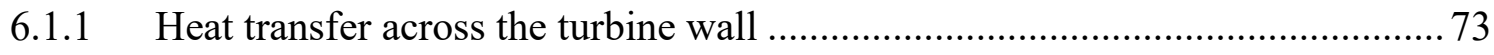

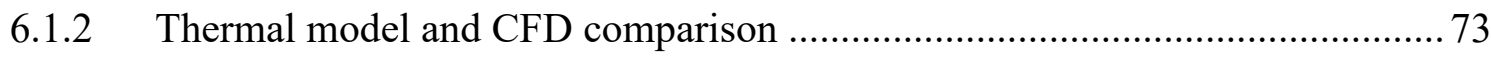

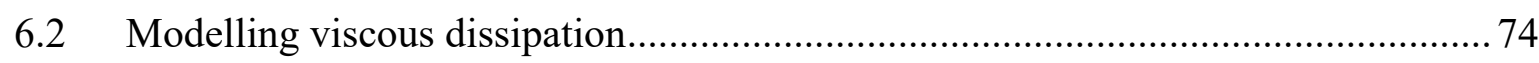

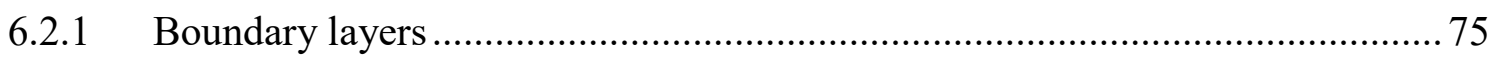

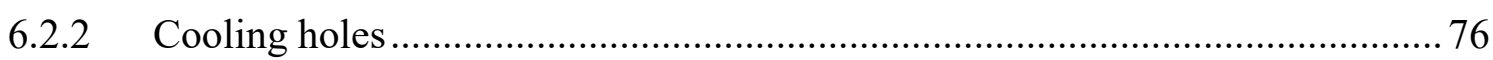

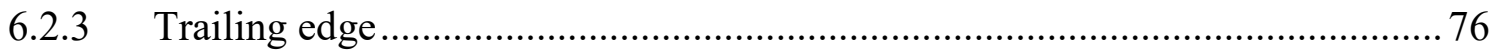

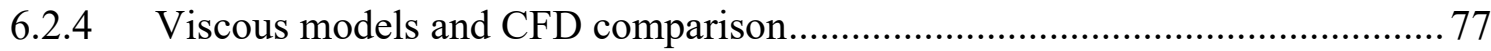

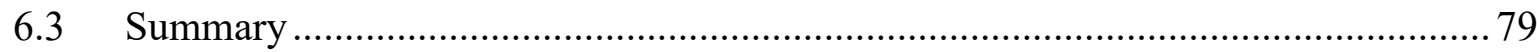

Chapter 7 Effect of heat transfer on boundary layer dissipation coefficient.................... 81

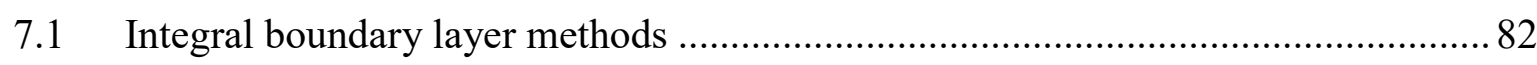

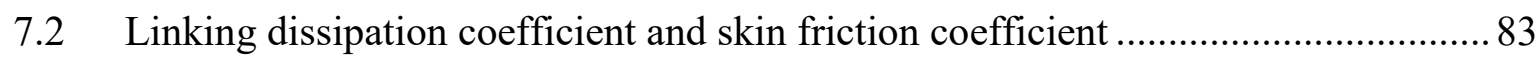

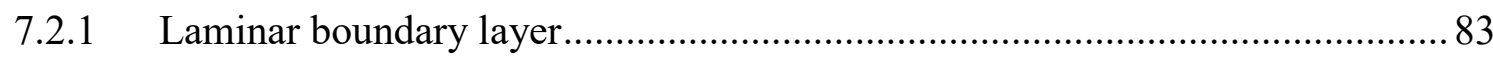

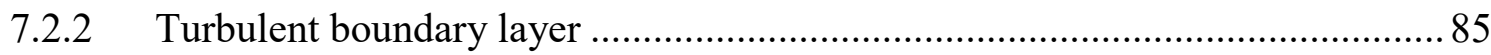

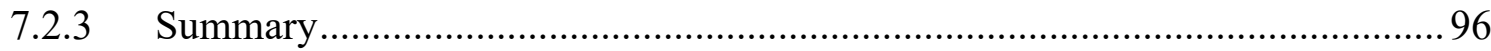

7.3 Linking dissipation coefficient to pressure-gradient coupling............................... 97

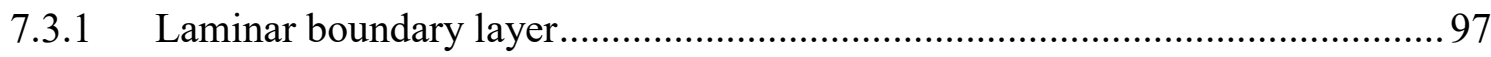

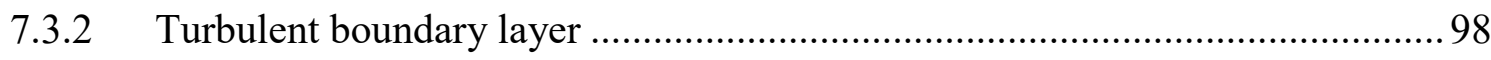

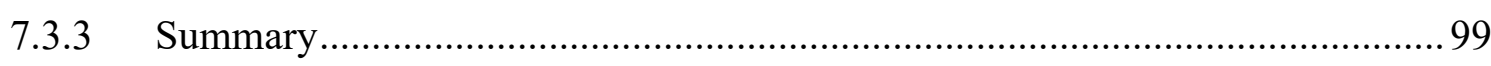

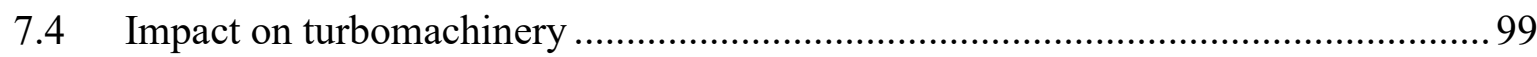

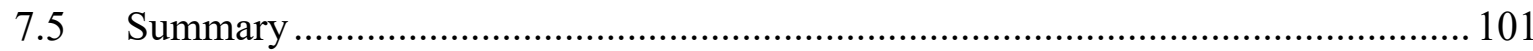

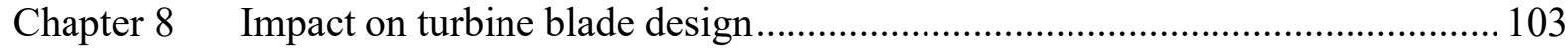

8.1 What is the aerothermal limit of thermal creation in a turbine cooling system? .... 104

8.2 What type of turbine cooling system maximises thermal creation? ....................... 107 
8.3 Can turbine loss coefficients be better translated from laboratory to engine? ....... 109

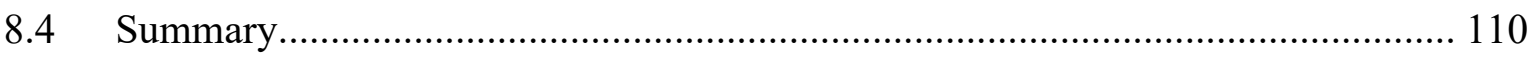

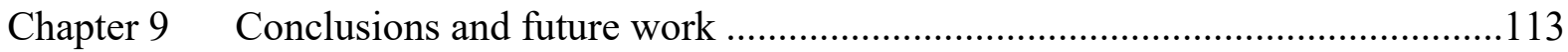

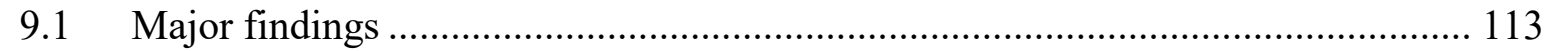

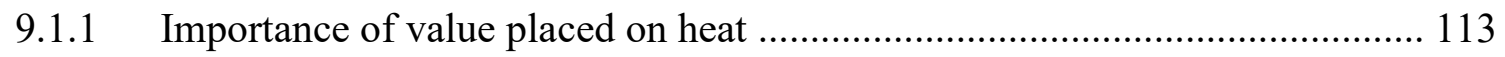

9.1.2 Effect of heat transfer on turbine loss mechanisms ..................................... 115

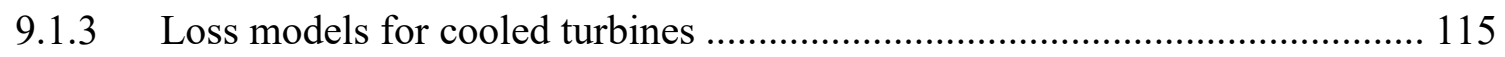

9.1.4 Effect of heat transfer on boundary layer dissipation coefficient ................... 116

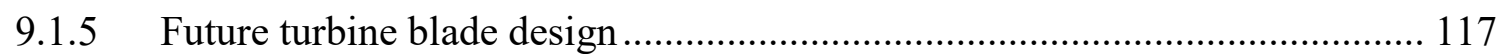

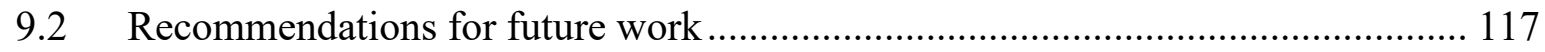

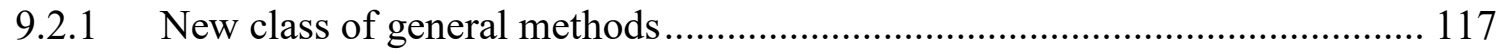

9.2.2 Linking heat transfer with thermodynamics ............................................. 118

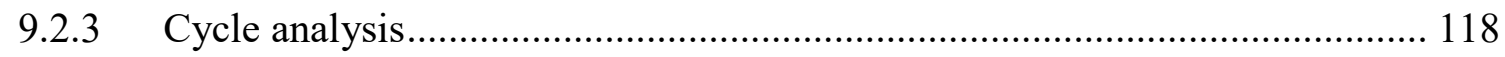

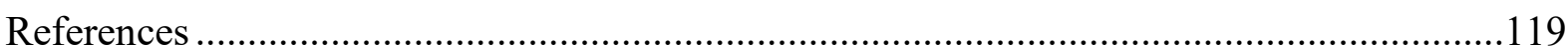

Appendix A General accounting methods ............................................................. A-1

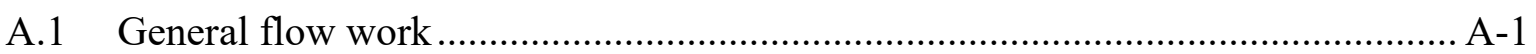

A.2 The effect of real gases on the euergy method ................................................... A-5

A.3 Comparing the euergy and power-balance methods .......................................... A-6

A.4 Comparing the euergy method and stagnation pressure loss.............................. A-8

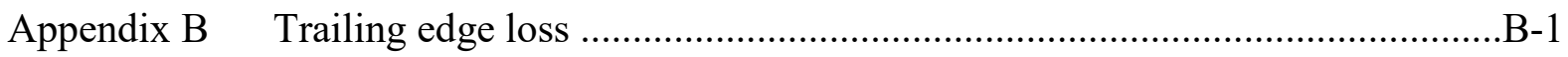

B.1 Kinetic energy loss coefficient decomposition................................................. B-1

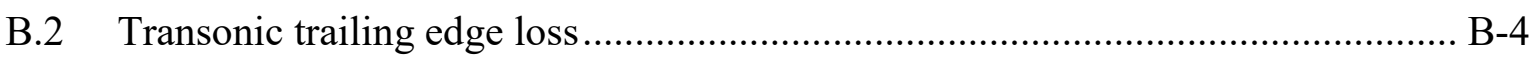

B.3 Linking the euergy loss coefficient to kinetic energy loss ................................ B-5

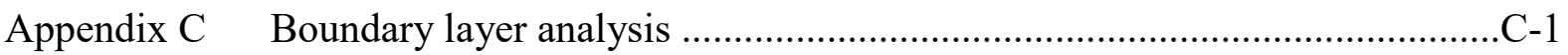

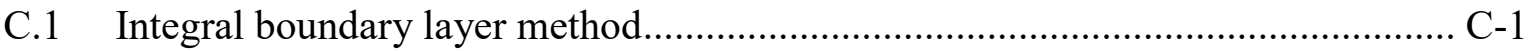

C.2 Compressible Blasius dissipation coefficient ................................................... C-5

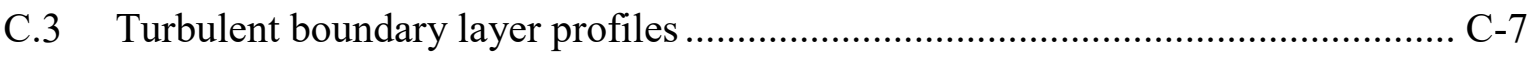

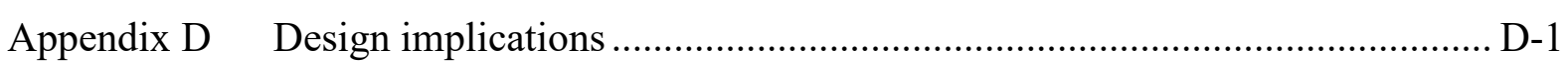

D.1 Steady one-dimensional flow euergy …...................................................... D-1 



\section{List of Figures}

Figure 1.1 - Heat transfer between the mainstream and coolant flow for a high-pressure turbine rotor blade (conjugate CFD solution).

Figure 2.1 - Enthalpy-entropy (h-s) diagram illustrating flow through an adiabatic turbine cascade from inlet, 1 , to exit, 2 .

Figure 2.2 - Temperature-entropy (T-s) diagram of flow through an adiabatic turbine cascade. Blue area indicates the "lost work" in the enthalpy loss coefficient. The red area is the difference in "lost work" between the entropy and enthalpy loss coefficients.

Figure 2.3 - Diagram illustrating the performance of a mixed turbine (left) and the mixing processes defining the fully-reversible, weighted-pressure and mainstream-pressure efficiencies (right).

Figure 2.4 - Schematic of a general method that determines the work out of the combined system based upon the heat and work interactions between any system and a reference environment.

Figure 2.5 - Boundary layer dissipation coefficient for self-similar flows at a Mach number, $\mathrm{M}$, of 0.7 .

Figure 3.1 - Mesh for simulating a film-cooled high-pressure turbine blade.

Figure 3.2 - Mesh independence study of internally-cooled (left) and film-cooled (right) CFD cases.

Figure 3.3 - Laminar and turbulent flow evaluated at $\mathrm{R} \delta 2=400$ and $\mathrm{R} \delta 2=1000$

respectively.

Figure 3.4 - Compressible Blasius solution viscosity ratio (left) and Chapman-Rubesin parameter, $C w$, (right) variation with mainstream-to-wall temperature ratio,....

Figure 3.5 - Uncertainty in dissipation coefficient due to the imbalance of the euergy equation in ANSYS Fluent simulations.

Figure 3.6 - Uncertainty in dissipation coefficient due to the imbalance of the euergy equation in TEXSTAN simulations.

Figure 3.7 - Mesh setup used to simulate flat plate boundary layer using 3DNS.

Figure 3.8 - Decay of freestream turbulence intensity, T, for 3DNS simulations................. 34

Figure 3.9 - Flat plate pressure gradient in 3DNS simulations.

Figure 4.1 - A cooled turbine loss coefficient can be calculated from the overall control volume. Currently, the contribution from a sub-control volume cannot be calculated. 
Figure 4.2 - Devices required to extract ideal work for the exergy method (left) and euergy

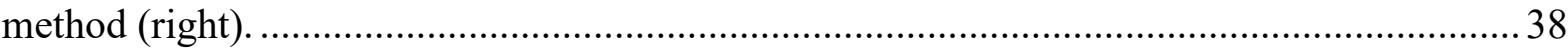

Figure 4.3 - Value of heat for the exergy method (a) and euergy method (b)...................... 39

Figure 4.4 - Exergy method to defining loss coefficient. ..................................................... 41

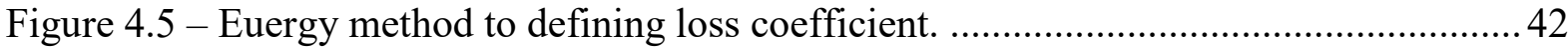

Figure 4.6 - The variation of exergy loss coefficient (red) and euergy loss coefficient (blue) with exit Mach number (left) and mainstream-to-coolant temperature ratio (right).............. 43

Figure 4.7 - The variation of euergy loss coefficient (blue) with exit Mach number compared to the power-balance loss coefficient (left) and the bound of the adapted power-balance loss coefficient (right).

Figure 4.8 - The variation of euergy loss coefficient (blue) with exit Mach number compared to the stagnation pressure loss coefficient (left) and the bound of the updated loss coefficient (right).

Figure 5.1 - The effect of transferring a small heat flux between two streams on the ideal work of a turbine.

Figure 5.2 - The variation of the euergy loss coefficient with mainstream-to-coolant temperature ratio.

Figure 5.3 - Contours showing the thermal creation contribution to turbine loss (temperature ratio of 2 ).

Figure 5.4 - The variation of the exergy loss coefficient with mainstream-to-coolant temperature ratio.

Figure 5.5 - Schematic showing viscous dissipation, such as that downstream of a throttle, is equivalent to work being removed from a flow and replaced by a heat addition of equal magnitude.

Figure 5.6 - The profile of Carnot cycle efficiency and Joule cycle efficiency for a cooled boundary layer.

Figure 5.7 - Contours showing the viscous dissipation contribution to turbine loss (temperature ratio of 2 ).

Figure 5.8 - The variation of exergy and euergy viscous loss coefficients with mainstream-tocoolant temperature ratio.

Figure 5.9 - Profiles of temperature, density, viscosity and viscous dissipation for a cooled boundary layer.

Figure 5.10 - The relative change in viscous loss coefficient with mainstream-to-coolant temperature ratio for fixed and variable thermofluid property simulations.

Figure 6.1 - Contours of the effect of thermal creation for the film-cooled blade (mainstreamto-coolant temperature ratio of 2).

Figure 6.2 - Decomposition of the effect of thermal creation on the loss coefficient of a filmcooled blade. 
Figure 6.3 - Contours of the effect of viscous dissipation for the film-cooled blade (mainstream-to-coolant temperature ratio of 2).

Figure 6.4 - The turbine loss coefficient divided into a control volume around the blade, CV1, and a control volume downstream of the trailing edge, CV2.

Figure 6.5 - Diagram of the film-cooled turbine blade divided into loss accounting regions. 78

Figure 6.6 - Decomposition of the effect of viscous dissipation on the loss coefficient of a film-cooled blade.

Figure 7.1 - The variation in dissipation coefficient (left) and skin friction coefficient (right) with mainstream-to-wall temperature ratio for laminar flow at $\mathrm{R} \delta 2=400$.

Figure 7.2 - The variation in dissipation coefficient (left) and skin friction coefficient (right) with mainstream-to-wall temperature ratio for turbulent flow at $\mathrm{R} \delta 2=1000$. ANSYS Fluent, blue; TEXSTAN, red; 3DNS, black; hollow marks, adiabatic; filled marks, isothermal.

Figure 7.3 - The variation in the ratio of dissipation and skin friction coefficients (left) and energy shape factor (right) with mainstream-to-wall temperature ratio for turbulent flow at $\mathrm{R} \delta 2=1000$. ANSYS Fluent, blue; TEXSTAN, red; 3DNS, black; hollow marks, adiabatic; filled marks, isothermal.

Figure 7.4 - The variation in component of dissipation coefficient due to mean (left) and fluctuating (right) velocities with mainstream-to-wall temperature ratio for turbulent flow at $\mathrm{R} \delta 2=1000$. ANSYS Fluent, blue; TEXSTAN, red; 3DNS, black; hollow marks, adiabatic; filled marks, isothermal.

Figure 7.5 - DNS normalised velocity profiles; traditional approach (left), semi-local scaling (right).

Figure 7.6 - DNS normalised dissipation profiles for mean dissipation and the production of

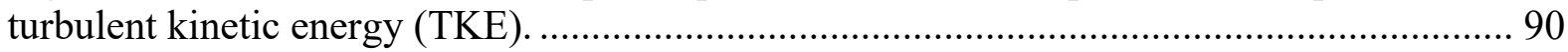

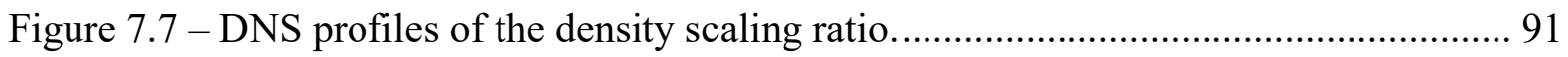

Figure 7.8 - DNS profiles of the relative change in length-scale. ...................................... 92

Figure 7.9 - DNS profiles of the overall scaling effect due to heat transfer.

Figure 7.10 - The ratio of the relative change in the effect of the production turbulent kinetic energy to the relative change in the effect of mean dissipation at different mainstream-to-wall temperature ratios.

Figure 7.11 - The effect of pressure gradient and mainstream-to-wall temperature ratio on laminar boundary layer flow at $\mathrm{R} \delta 2=400$. Left, dissipation coefficient. Right, skin friction coefficient.

Figure 7.12 - The effect of pressure gradient and mainstream-to-wall temperature ratio on turbulent boundary layer dissipation coefficient at $\mathrm{R} \delta 2=1000$ predicted using different turbulence models. Top left, mixing-length. Top right, Hassid \& Poreh. Bottom left, Launder \& Sharma. Bottom right, Jones \& Launder.

Figure 7.13 - The change in blade loss coefficient with mainstream-to-coolant total temperature ratio using different turbulence models in ANSYS Fluent. 
Figure 8.1 - Contours showing the ratio of the effect of thermal creation to the effect of viscous dissipation as the Mach number of the mainstream and coolant are varied. The values are for the turbine stator blade (left) and the turbine rotor blade (right) presented by Young \& Wilcock (2002b).

Figure 8.2 - Fractional change in loss coefficient due to thermal creation for film-cooling (blue), internal-cooling (red) and the internal-cooling upper limit (grey)

Figure 8.3 - The change in loss coefficient between a film-cooled and adiabatic blade due to the boundary layer, cooling holes, wake and thermal creation. 109

Figure 9.1 - Heat transfer across a difference in Joule cycle efficiency (conjugate CFD solution). 


\section{List of Tables}

Table 3.1 - Summary of mesh sizes used for simulating internally-cooled and film-cooled

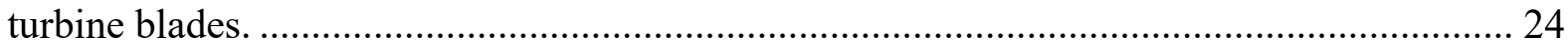

Table 3.2 - Summary of flow setup for all turbine simulations, unless otherwise stated....... 24

Table 3.3 - Summary of CFD setup for all turbine blade simulations, unless otherwise stated.

Table 3.4 - Summary of laminar and turbulent boundary layer flow conditions.

Table 3.5 - Summary of $1^{\text {st }}$ cell $y+$ at $R \delta 2=1000$ used for ANSYS Fluent boundary layer

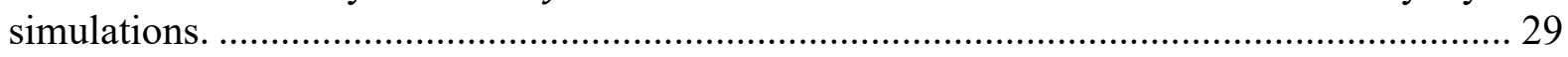

Table 3.6 - Summary of ANSYS Fluent setup used for boundary layer simulations. ........... 29

Table 3.7 - Summary of TEXSTAN setup used for boundary layer simulations................... 31

Table 3.8 - Summary of $1^{\text {st }}$ cell $y+$ at $R \delta 2=1000$ used for 3DNS boundary layer simulations

Table 3.9 - Uncertainty in dissipation coefficient due to the imbalance between production

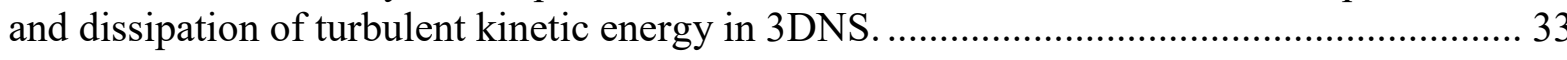

Table 3.10 - Summary of the 3DNS setup used for boundary layer simulations. ................. 35

Table A.1 - Thermal efficiencies and pressure equilibrium coefficients for general methods.

Table A.2 - Gas compression parameters. 



\title{
Nomenclature
}

\author{
Acronyms/Abbreviations
}

ARCHER Academic Research Computing High End Resource.

BL Boundary layer.

CFD Computational fluid dynamics.

$\mathrm{CH} \quad$ Cooling holes.

CSD3 Cambridge Service for Data Driven Discovery.

$\mathrm{CV} \quad$ Control volume.

DNS Direct Numerical Simulation.

FR Fully-reversible.

HTC Heat transfer coefficient.

IBLM Integral boundary layer method.

MP Mainstream-pressure.

MUSCL Monotonic Upwind Scheme for Conservation Laws.

PS Pressure-side.

RANS Reynolds-averaged Navier-Stokes.

SL Semi-local.

SS Suction-side.

TE Trailing-edge.

TKE Turbulent kinetic energy.

URANS Unsteady Reynolds-averaged Navier-Stokes.

WP Weighted-pressure. 


\section{Greek symbols}

$\alpha$

$\beta$

$\gamma$

$\delta$

$\delta_{1}$

$\delta_{2}$

$\delta_{3}$

$\delta_{\rho}$

$\delta_{H}$

$\Delta_{\text {therm }}$

$\epsilon$

$\eta_{c}, \eta_{t}$

$\eta_{t h}$

$\lambda$

$\Lambda$

$\mu$

$v=\mu / \rho, v=1 / \rho$

$\pi$

$\Pi$

$\rho$

$\sigma$

$\Sigma$

$\tau$

$\phi$

$\phi_{v}$

Mesh expansion ratio or coolant ejection angle.

Pressure-gradient: Laminar, Blasius parameter;

Turbulent, Rotta-Clauser parameter.

Ratio of specific heats.

Infinitesimal amount.

Displacement thickness.

Momentum thickness.

Energy thickness.

Density thickness.

Enthalpy thickness.

Thermal creation rate per unit volume.

Dissipation of turbulent kinetic energy.

Isentropic efficiency of a compressor and turbine respectively.

Local thermal efficiency (i.e. value placed on heat relative to work).

Relative change in length-scale.

Pressure-gradient parameter.

Absolute viscosity.

Kinematic viscosity or specific volume.

Production of turbulent kinetic energy profile.

Contribution to dissipation coefficient due to fluctuating velocity.

Density.

Exponent for determining Blasius solution viscosity.

Sum of all inlets or outlets.

Shear-stress.

Mean dissipation profile.

Viscous dissipation. 
$\phi_{t h}$

$\phi_{\text {therm }}$

$\Phi$

$\Phi_{v}$

$\Phi_{t h}$

$\omega$

$\Omega$

\section{Roman symbols}

$a$

$c_{d}$

$c_{f}$

$c_{p}$

$c_{p b}$

$C_{p}^{e q}$

$C_{w}$

D

$d S$

$d \mathrm{~V}$

$\mathbf{e}_{\mathbf{f}}$

f

$h$

$H_{12}=\delta_{1} / \delta_{2}$

$H_{32}=\delta_{3} / \delta_{2}$

$H_{\rho 2}=\delta_{\rho} / \delta_{2}$

$k$

$\dot{K E}$

$l$
Thermal recuperation effect.

Thermal dissipation.

Contribution to dissipation coefficient due to mean velocity.

Viscous loss mechanism.

Thermal loss mechanism.

Loss coefficient.

Mainstream-to-wall temperature ratio.

Speed of sound.

Dissipation coefficient.

Skin-friction coefficient.

Specific heat.

Base pressure coefficient.

Pressure equilibrium coefficient.

Chapman-Rubesin parameter.

Diameter.

Surface element of control volume.

Volume element of control volume.

Flow exergy per unit mass.

Friction value.

Enthalpy.

First shape factor of velocity profile.

Second shape factor of velocity profile.

Shape factor of density profile.

Turbulent kinetic energy.

Flux of kinetic energy.

Mixing length. 


\begin{tabular}{|c|c|}
\hline$L$ & Length. \\
\hline$\dot{m}$ & Mass flow rate. \\
\hline M & Mach number. \\
\hline$m_{f}$ & Flow euergy per unit mass. \\
\hline$\hat{n}$ & Unit normal vector, out of control volume. \\
\hline$p$ & Static pressure. \\
\hline$P$ & Prandtl number. \\
\hline$p_{f}$ & Flow power-balance per unit mass. \\
\hline$\vec{q}$ & Heat flux vector. \\
\hline$\dot{Q}$ & Rate of heat. \\
\hline$r$ & Recovery factor. \\
\hline $\mathrm{R}$ & Reynolds number. \\
\hline$R$ & Gas constant. \\
\hline$s$ & Entropy. \\
\hline$S_{\text {blade }}$ & Blade surface. \\
\hline$t$ & Blade trailing edge thickness. \\
\hline$T$ & Static temperature. \\
\hline $\mathrm{T}$ & Turbulence intensity. \\
\hline$u, U$ & Velocity component parallel to the wall. \\
\hline$u_{\tau}=\sqrt{\tau_{w} / \rho_{w}}$ & Friction velocity. \\
\hline$\vec{V}$ & Velocity vector. \\
\hline$w$ & Throat width. \\
\hline$\dot{W}$ & Rate of work. \\
\hline $\mathbf{w}_{\mathbf{f}}$ & Ideal flow work per unit mass. \\
\hline$x, y, z$ & Physical dimensions \\
\hline$x^{+}, y^{+}, z^{+}$ & Normalised dimensions. \\
\hline$y_{f}$ & Flow stagnation-pressure-loss per unit mass. \\
\hline$Y^{+}$ & Dimensionless wall distance. \\
\hline
\end{tabular}


Blade span in CFD solution.

\section{Subscript}

$\begin{array}{ll}{[]_{\mathrm{aw}}} & \text { Adiabatic wall value. } \\ {[]_{\text {coolant }},[]_{c}} & \text { Value in the coolant flow. } \\ {[]_{\mathrm{ex}}} & \text { Exhaust value. } \\ {[]_{\text {ideal }}} & \text { Value corresponding to an ideal process. } \\ {[]_{\mathrm{in}}} & \text { Inlet. } \\ {[]_{\mathrm{IO}}} & \text { Inlets and outlets. } \\ {[]_{\mathrm{mainstream}},[]_{m}} & \text { Value in the mainstream flow. } \\ {[]_{\mathrm{mix}}} & \text { Value at a mixed state. } \\ {[]_{\mathrm{out}}} & \text { Outlet. } \\ {[]_{\mathrm{ref}}} & \text { Reference value. } \\ {[]_{s, p_{0}}} & \text { Value defined by an isentropic expansion to environment pressure. } \\ {[]_{\mathrm{SS}}} & \text { Suction-side value } \\ {[]_{\mathrm{t}}} & \text { Total quantity. } \\ {[]_{\mathrm{te}}} & \text { Value at the trailing edge. } \\ {[]_{\mathrm{tr}}} & \text { Value at a transformed state. } \\ {[]_{\mathrm{Trefft}}} & \text { Value at a Trefftz plane. } \\ {[]_{\mathrm{wall}},[]_{w}} & \text { Value at the wall. } \\ {[]_{0}} & \text { Value at reference environment (dead-state). } \\ \end{array}$

\section{Superscript}

$\overline{[}^{\mathrm{a}}$

$\overline{[}^{\mathrm{m}}$
Area-averaged quantity.

Mass-averaged quantity. 



\section{Chapter 1 Introduction}

For over 60 years, gas turbines have bled air from the compressor to cool blades in the highpressure turbine. The cooling air is used to protect these turbine blades, allowing them to operate at temperatures several hundred degrees above their melting point. At the cycle level, the increase in operating temperature permits a greater cycle efficiency to be achieved. At the component level, this cooling results in very high rates of heat transfer - both within the fluid and within the blade. It is therefore surprising that little is understood about the effect of heat transfer on turbine efficiency. There is not even a consensus on how to define the efficiency of a cooled turbine.

The effect of cooling on turbine performance has been investigated in several important studies (Hawthorne 1956; Denton 1993; Horlock 2001; Young \& Wilcock 2002; Young \& Horlock 2006). All these studies consider the effect of heat transfer at the component level, and then apply a thermodynamic method to account for the change in performance at the cycle level. Linking performance between different levels of design enables designers to optimise their component, such as a turbine blade, to maximise the efficiency of the overall gas turbine. However, these studies achieve this link by applying a thermodynamic method that has only been validated by considering simple cycles. When extended to more realistic cooled systems, turbine performance is no longer reflected.

The thermodynamic method these studies adopted is based upon entropy creation. Whenever entropy is created due to irreversible processes within the turbine, the method predicts a reduction in performance. In a cooled turbine, the entropy created due to heat transfer between the hot mainstream flow and the cooler coolant flow (Figure 1.1) is extremely large (i.e. enough to suggest that the turbine efficiency drops by around 4-6\%). In practice, this drop in efficiency is not observed. In fact, industrial designers are so confident that heat transfer does not have a large effect on performance that they measure the efficiency of cooled turbines 


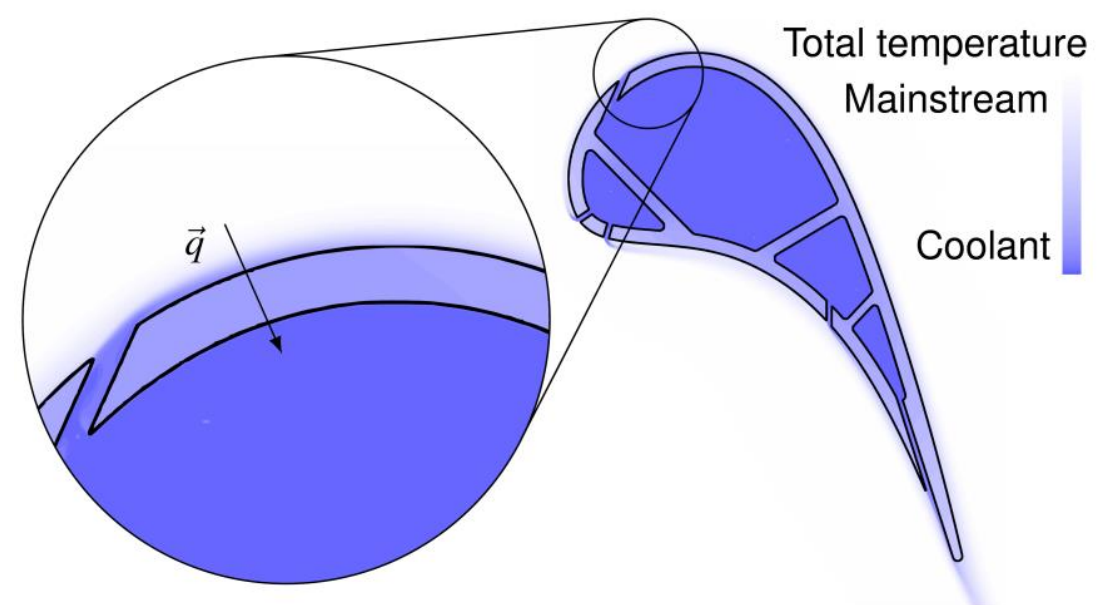

Figure 1.1 - Heat transfer between the mainstream and coolant flow for a high-pressure turbine rotor blade (conjugate CFD solution).

using isothermal turbine test facilities (i.e. with the mainstream and coolant artificially set to the same temperature). The direct contradiction between this entropy-centric method and the experience of turbine designers can be explained for simple examples but no solution exists for more complex, engine-representative cases.

This thesis seeks to address this contradiction and establish a thermodynamic method capable of both linking different levels of design and agreeing with industrial experience.

\subsection{Defining cooled turbine performance}

"Efficiency is probably the most important performance parameter for most turbomachines" (Denton 1993). This is for good reason as it is particularly useful for two purposes. In engine performance or advanced project offices, the main use is to predict the actual power that will be generated. In turbine design offices, the primary use is to compare between different turbine designs. Efficiency can be communicated between all levels of design provided it has a clear, unambiguous definition. Although this can be achieved for uncooled turbines, there is no agreement on how to best define the efficiency of a cooled turbine.

As highlighted by Young and Horlock (2006), the main problem in defining cooled turbine efficiency is specifying the ideal work that could be extracted by a turbine. The ideal 
work can be determined by comparing the flow state, which is known, with some ideal exhaust state, which is not.

Consider the flow through an adiabatic turbine with no cooling or purge flows. For this uncooled turbine, there is only one stream which allows the ideal exhaust state to be calculated using a single reversible adiabatic expansion to the exit pressure. However, a cooled turbine has multiple different streams and flow states. This makes finding the ideal exhaust state, and consequently the ideal work, unclear.

\subsection{Loss mechanisms in cooled turbines}

Denton (1993) describes loss as any flow feature that reduces the efficiency of a turbomachine. These flow structures occur within a component but impact the overall efficiency at the cycle level. Inherent in the definition of loss is the link between the different design levels; linking effects at the component level to their impact at the cycle level.

To establish this link, many studies use a thermodynamic method based upon entropy creation. Entropy that is created due to irreversible processes within the turbine is used to measure the reduction in its performance. The irreversible flow processes could be due to viscous dissipation, thermal dissipation or chemical dissipation. These occur from mixing together streams of different velocities, temperatures or chemical compositions respectively.

For uncooled turbines, viscous dissipation is responsible for most of the entropy creation. Viscous dissipation is the process by which streams of different velocities equilibrate. This produces entropy is in regions of steep velocity gradients, such as boundary layers, wake mixing and shock waves. Viscous dissipation is often divided into a laminar (molecular) and turbulent contribution. The reduction in performance predicted by this rise in entropy agrees with industrial experience.

For cooled turbines, thermal dissipation contributes significantly to the overall entropy creation. Thermal dissipation occurs when streams of different temperatures equilibrate. This occurs when heat is transferred across differences in temperature, both within the fluid and within the blade. Although the relative magnitude is smaller, thermal dissipation also occurs in 
uncooled turbines where there is a difference between the temperature of the oncoming flow and the blade surface. The reduction in performance predicted for cooled turbines does not agree with industrial experience.

It is currently unclear how to resolve this contradiction between entropy-centric methods and industrial experience. A popular suggestion is that only the entropy created due to viscous dissipation is responsible for reducing the efficiency of a turbomachine. This explanation can be supported by considering simple cycles, but not for more complex, enginerepresentative cases. It is unclear what the loss mechanisms are for cooled turbines.

\subsection{Practical measurement of cooled turbine performance}

In the engine, high-pressure turbine blades operate at very high temperatures which makes them difficult to instrument. To measure their performance, these turbine blades are typically tested in a laboratory at much lower temperatures. The aerodynamic conditions experienced by the turbine blades are recreated by matching the appropriate parameters governing the flow. However, the lower temperatures mean that the heat transfer rates are not recreated.

The loss coefficient determined in this laboratory setting is then translated to engine conditions. Only the change in temperature of the air leaving the cooling holes is corrected for in this translation - the effect of heat transfer is not considered. This implies that industry currently believe that heat transfer does not significantly affect turbine performance.

\subsection{Thesis structure}

After a review of the literature (Chapter 2) and details of the computational methods (Chapter $3)$, the major results of this thesis are grouped in 3 themes:

The development of a thermodynamic method to define cooled turbine performance. Chapter 4 seeks to establish a method to resolve the contradiction between entropy-centric methods and industrial experience. The aerodynamic performance accounting methods currently applied across industry and academia are compared to identify their key differences 
and the limits of their applicability. With better understanding, the traditional metrics for uncooled turbines can be extended to cooled turbines. In addition, turbine performance can be linked between the different levels of design in a way that reflects industry experience.

The effect of heat transfer on loss in cooled turbines. The aims of Chapter 5, Chapter 6 and Chapter 7 are to explain the effect of heat transfer on turbomachinery loss mechanisms. A novel thermodynamic method is applied to examine the loss mechanisms around a cooled turbine blade. This facilitates simplified models to be developed that accurately capture these loss mechanisms, which are presented in Chapter 6. These are useful as they distil the effect of heat transfer into tools that allow designers to make fast and informed decisions.

The implications of this new understanding on turbine design. This thesis presents a new perspective on how heat transfer affects turbine performance, which has implications for industrial design. Chapter 8 considers some of these implications through three practical questions. 



\section{Chapter 2 Literature review}

The purpose of this thesis is to investigate how heat transfer affects the performance of a cooled turbine. In this chapter, the aim is to establish the limit of current knowledge. The extent of current knowledge is reviewed in five topics and culminates in five research questions.

The first section examines how turbine performance is currently defined. A turbine cascade is used to demonstrate the metrics of performance applied to uncooled blades. This section seeks to outline the challenges of extending established methods to cooled turbines.

The second section reviews current methods of performance accounting. These methods quantitatively link local flow processes within a component to their impact on the overall performance. The aim of this section is to establish the current applications, the extent of use and the known limitations of these methods.

The third section explores how to develop simplified models for loss in turbomachinery. Loss models distil complex flow physics into an easily calculable tool to help designers make fast and informed decisions. This section targets current turbomachinery loss modelling techniques focussing on their applicability to cooled turbines.

The fourth section investigates how heat transfer affects flow in the boundary layer. For a cooled turbine, the mechanism responsible for most of the heat transfer is also responsible for momentum transfer. The goal of this section is to determine how this aerothermal coupling may affect turbine performance.

The fifth section reviews some of the compromises in practical cooled turbine design. The optimal cooling design is a balance between aerodynamic performance and mechanical constraints. This section seeks to summarise the principle trade-offs when designing a real turbine. 


\subsection{Defining turbine performance}

A clear and unambiguous definition of turbine performance is required to improve communication between different levels of design. In this section, methods of determining the performance of both uncooled and cooled designs are examined by considering a turbine cascade.

For an uncooled turbine cascade, the expansion of the flow can be illustrated using an enthalpy-entropy (h-s) diagram as shown in Figure 1.1. The appropriate metric of performance for a cascade is known as a loss coefficient. This represents the ratio of "lost work", $\dot{W}_{\text {lost }}$, to a flux of kinetic energy, $\dot{K} E_{\text {exit }}$. The "lost work" is calculated from the change in ideal work that could be extracted at the inlet and exit. There are several different loss coefficients available depending on how the ideal work is calculated. These have been reviewed by Brown (1972). In this thesis, three commonly applied loss coefficients for adiabatic turbine cascades will be considered. These are the enthalpy loss coefficient, $\omega^{h}$, the entropy loss coefficient, $\omega^{s}$, and the stagnation pressure loss coefficient, $\omega^{p}$ which are expressed in Equation 2.1. These are the same coefficients considered by Denton (1993).

$$
\omega^{h}=\frac{h_{2}-h_{2 s}}{h_{t, 2}-h_{2}}, \quad \omega^{s}=\frac{T_{2}\left(s_{2}-s_{1}\right)}{h_{t, 2}-h_{2}}, \quad \omega^{p}=\frac{p_{t, 1}-p_{t, 2}}{p_{t, 2}-p_{2}}
$$

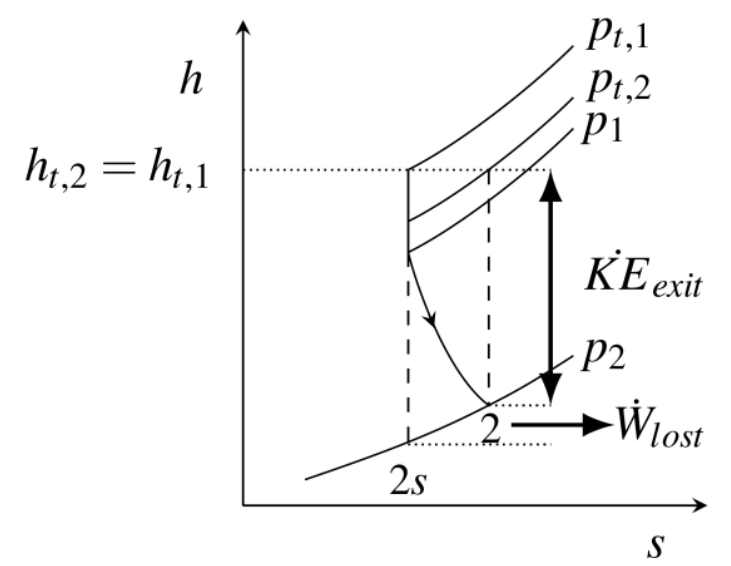

Figure 2.1 - Enthalpy-entropy (h-s) diagram illustrating flow through an adiabatic turbine cascade from inlet, 1 , to exit, 2. 


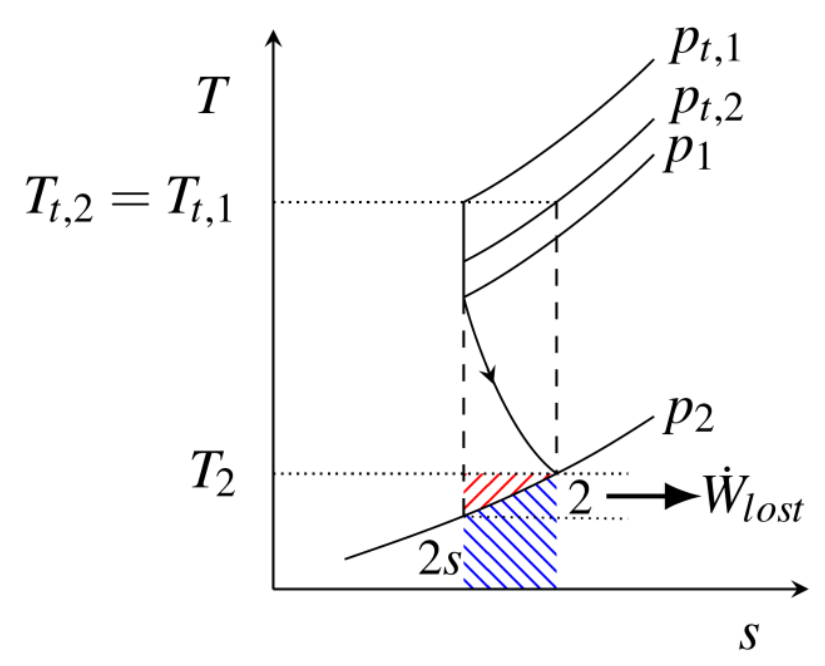

Figure 2.2 - Temperature-entropy (T-s) diagram of flow through an adiabatic turbine cascade. Blue area indicates the "lost work" in the enthalpy loss coefficient. The red area is the difference in "lost work" between the entropy and enthalpy loss coefficients.

The enthalpy loss coefficient, sometimes known as the energy loss coefficient, can be derived by considering the change in work that an ideal turbine could extract between the inlet and exit. The process for extracting this ideal work is an isentropic expansion to the turbine exit pressure. As the flow passes through the turbine, entropy is produced which acts to change the exhaust state of the ideal turbine. This change in exhaust state reduces the work that the turbine could extract. The reduction in ideal work, or "lost work", is graphically depicted by the blue area under the exhaust pressure line in Figure 2.2.

The entropy loss coefficient calculates the ideal work by considering the difference in the thermodynamic states of the flow and the actual exhaust. A consequence of this is that all entropy produced in the turbine cascade is equally deleterious. As irreversible processes can only create entropy, Denton (1993) introduced a smoke analogy. Once created, entropy is convected downstream like smoke. The average concentration of this "smoke" at the exit is proportional to the reduction in ideal work. Graphically, this is depicted as the sum of both the red and blue areas in the Figure 2.2. 
The difference between the enthalpy and entropy loss coefficients is due to the exhaust temperature at which they value entropy, as shown by Equation 2.2. The enthalpy loss coefficient values entropy based upon the exhaust temperature a local ideal turbine could achieve. This temperature varies throughout the cascade, leading to the blue area in Figure 2.2. The entropy loss coefficient values entropy based upon the final exhaust temperature achieved by the actual device. This temperature is fixed, leading to the red area in Figure 2.2 also being included.

$$
\omega^{h}=\frac{\int T_{s, p_{2}} d s}{h_{t, 2}-h_{2}}, \quad \omega^{s}=\frac{T_{2} \int d s}{h_{t, 2}-h_{2}}
$$

For small changes in the exhaust temperature, the enthalpy and entropy loss coefficients are similar with the red area becoming small. For adiabatic cascades, Denton (1993) gave the difference between the enthalpy and entropy loss coefficients as Equation 2.3. In practice, the difference is negligible for most uncooled cascades.

$$
\omega^{h}-\omega^{s}=0.25(\gamma-1) \mathrm{M}^{2} \omega_{h} \omega_{s}
$$

Both enthalpy and entropy are not the most intuitive quantities as they cannot be seen and are challenging to measure directly. This is a key reason behind the prevalence of the third metric.

The stagnation pressure loss coefficient estimates the change in ideal work that a turbine could extract exhausting to a constant pressure. The accuracy of this estimate stems from the link between the change in total pressure and the change in mechanical energy per unit volume. However, this link it only valid in the limit of low Mach number flow. Denton (1993) suggested that the error only becomes significant at Mach numbers greater than about 0.3 .

At greater Mach numbers, the stagnation pressure measurements can be used to calculate the entropy loss coefficient for uncooled cascades. This is because, for a simple compressible perfect gas, entropy is a function of any two independent thermodynamic 
properties. As the change from static to stagnation quantities is isentropic, differences in entropy can be written in terms of stagnation pressure and stagnation temperature. For an uncooled cascade, changes in stagnation pressure correspond to changes in entropy. This links the stagnation pressure loss coefficient to the entropy loss coefficient.

For an uncooled turbine cascade, all three loss coefficients have a clear interpretation of ideal work. The ideal work is calculated by comparing the thermodynamic state of the flow to an ideal exhaust. The relationship between the coefficients is well-understood and, at low Mach numbers, they all reflect turbine performance. Turbine performance can even be captured at high Mach numbers using the enthalpy or entropy loss coefficients. However, it is unclear how to extend these loss coefficients to cooled turbines.

For a cooled turbine, Young \& Horlock (2006) highlighted that the main difficulty in defining performance is specifying the ideal work that could be achieved. As before, the ideal work is calculated by comparing the thermodynamic state of the flow to an ideal exhaust. However, the number of different streams and range of thermodynamic states within a cooled turbine makes it difficult to define an ideal exhaust state.

To achieve a single exhaust state, Young \& Horlock (2006) proposed 3 efficiencies based upon different mixing processes; fully-reversible (FR), weighted-pressure (WP) and mainstream-pressure (MP) efficiencies. The ideal work is then obtained by a single reversible adiabatic expansion to these different mixed out states as illustrated, in Figure 2.3.

The aim of the efficiencies proposed by Young \& Horlock (2006) was to provide clear and thermodynamically-grounded definitions. Despite the progress in defining cooled turbine performance, these metrics have not been widely adopted for two reasons. The first is that, although the definitions are unambiguous, it is not clear which efficiency best reflects the ideal work of a practical turbine. Secondly, it is not clear which flow processes are responsible for reducing these efficiencies. 

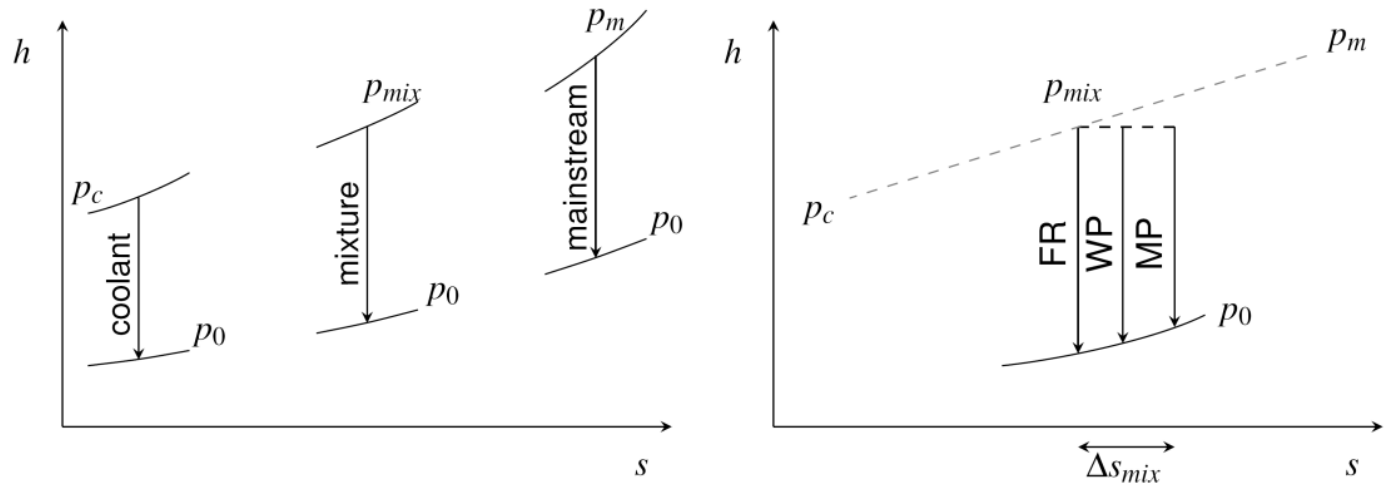

Figure 2.3 - Diagram illustrating the performance of a mixed turbine (left) and the mixing processes defining the fully-reversible, weighted-pressure and mainstream-pressure efficiencies (right).

There have been several attempts to relate the performance of a cooled expansion to an equivalent uncooled version. Casey (2007) focuses on accounting for the effect of heat transfer on the expansion process using an adapted polytropic efficiency. This approach models the thermodynamic expansion, but does not indicate which flow processes were responsible. Atkins \& Ainsworth (2012) calculate an adiabatic efficiency from non-adiabatic testing by estimating the entropy change of the exhaust due to heat transfer. This study demonstrated that the main effect of heat transfer out of the control volume is to reduce the work potential of the flow and that this can be corrected for by examining the change in exhaust state. The effect of both viscous and thermal irreversibilities are still present in their final definition as only the effect of reversible heat transfer out of the control volume is correct for. In addition, the use of total quantities in their approximations may be a reasonable approximation in this work but this error would need further examination before being applied more generally. This approach should be considered a practical method to correct for the change in entropy due to reversible heat transfer out of a control volume in an experimental setting.

To summarise, a clear understanding of ideal work is required to define turbine performance. For an uncooled turbine, this can be achieved as both the thermodynamic states of the flow and exhaust are clearly defined. However, this understanding is difficult to extend to cooled turbines as it is unclear how to define the exhaust state. Young and Horlock (2006) proposed 3 mixing processes that could achieve this, but it is not certain if they reflect industry experience. It remains unclear how to define the performance of a practical cooled turbine. 


\subsection{General methods of performance accounting}

Aerodynamic performance accounting methods are widely used to identify avenues of improvement and to rank designs. Historically, this has been achieved by analysing a control volume placed around a component of interest. By examining the changes on the surface of this control volume, the overall effect of the flow processes within could be inferred. For instance, the loss coefficients introduced in the previous section measure values at the inlet and exit to infer the effect of loss within the cascade. However, this approach does not inform a designer where these flow processes took place - where loss originated. A method that can link local flow processes to their effect on the boundaries of the control volume for any system will be referred to as a general method.

Based upon the study by Keenan (1951), a general method considers the first and second laws of thermodynamics for processes between equilibrium states of a system operating within an infinite stable atmosphere. From such considerations, concepts such as maximum useful work, availability, irreversibility and performance metrics can be defined. This generality allows a wide variety of systems to be examined, including turbomachinery.

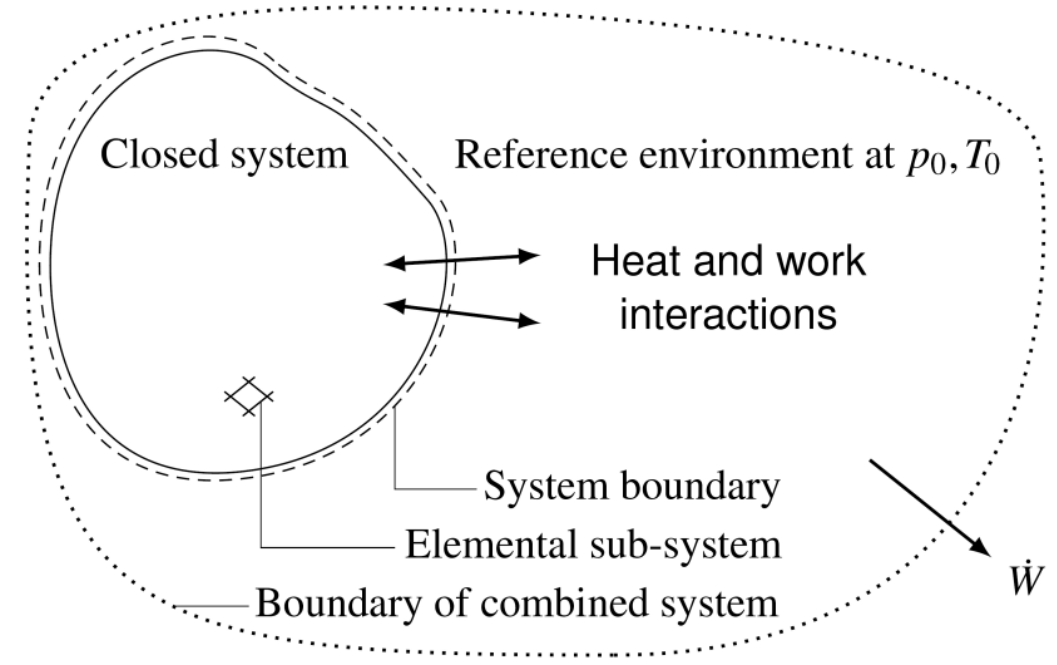

Figure 2.4 - Schematic of a general method that determines the work out of the combined system based upon the heat and work interactions between any system and a reference environment. 
Figure 2.4 illustrates how a general method links the amount of work that can be extracted from the combined system to changes due to heat and work interactions. These interactions occur between the system and the reference environment and their nature distinguishes between general methods. By restricting the interactions based upon a thermodynamic process that reflects ideal work, information about the practical device can be embedded.

An example of this is the exergy method. This is an established method that examines the maximum available work that could be extracted from the combined system using all forms of reversible heat and work interactions. As the exergy method is general, the effect of local flow processes occurring in an elemental sub-system are inherently linked to their effect on the combined system. This provides a thermodynamic method that can link across all levels of design.

The rigorous nature of generalizable methods has led exergy-based methods to be used throughout the turbomachinery community, with best practices outlined by Moran \& Sciubba (1994). These methods extend the entropy loss coefficient to analyse local flow processes and share the view that all entropy creation is equally deleterious. For this reason, exergy-based methods were referred to as entropy-centric methods in the introduction. While they have had success for simple devices, they do not reflect turbine performance observed by industry.

Due to the discrepancy between the exergy method and industrial experience, several new performance accounting methods have been recently developed. These methods aim to better reflect the performance to which the practical system aspires. Two such methods will be considered.

The first is the euergy method, or mechanical work potential method, proposed by Miller (2013). This is another general method and was developed to account for turbomachinery performance. It has been derived by considering the ideal amount of work that could be extracted from the flow using a reversible adiabatic expansion. Consequently, euergy appears well-suited to examining turbomachinery flow as it has been derived from the same thermodynamic process to which turbomachines aspire - a reversible adiabatic expansion. To the author's knowledge, this method has only previously been applied by Blackburn \& Miller 
(2017) to examine pressure gain combustion and Leggett et al. (2019) to examine unsteady compressor flow.

The second is the power-balance method proposed by Drela (2009). It was developed to account for propulsor-airframe integration in future aircraft concepts. The power-balance method can be derived by placing a control-volume in the freestream around the aircraft. The downstream plane is then related to a Trefftz plane representing the freestream pressure and velocity.

For turbomachinery applications, the Trefftz plane concept of a uniform freestream pressure and velocity is not well suited. By viewing the Trefftz plane conditions as a reference environment, the power balance method can be generalised for any system. Although this method seems unlikely to represent turbomachinery applications, the limitations of the power balance method are not known.

Comparing between all three methods, there are two categories of aerodynamic performance accounting method. There are the general methods, exergy and euergy, and there are the industrially-focussed methods, euergy and power-balance method. In this thesis, industrially-focussed methods are those that have been developed to address a particular application. The limitations of all three methods for examining turbomachinery flows are not known. They all can link local flow processes to their effect on performance, but it is unclear which best reflects industrial experience.

\subsection{Modelling loss mechanisms in turbomachinery}

Denton (1993) described loss as any flow feature that reduced the efficiency of a turbomachine. These flow features, or loss mechanisms, occur at the component design level but impact the overall efficiency at the cycle level. Inherent in the definition of loss is the link between different levels of design.

As an aside, it is worth noting that many factors change the overall efficiency but only those due to local flow features are considered loss mechanisms. For instance, factors that occur at the cycle level, such as changes in engine architecture, would not be a loss mechanism. 
Linking loss mechanisms to their effect on performance has been achieved by examining a control-volume placed around a component of interest. After this, there are two ways of identifying loss mechanisms; either decomposing a performance metric, such as a loss coefficient, or by applying an aerodynamic performance accounting method.

By decomposing a loss coefficient, the effect of a loss mechanism can be inferred by examining the control-volume surface. Using this technique, Denton \& Xu (1989) were able to examine trailing edge loss mechanisms by decomposing the stagnation pressure loss coefficient of a transonic turbine. This approach can be successful, but the decomposition is both subjective and specific for each application. This approach is reliant upon validation to ensure the decomposition has produced physically meaningful parameters that are aligned with the practical device.

This practical approach derives from cascade experiments. Other examples include Soderberg (1949), Ainley \& Mathieson (1951), Dunham \& Came (1970), Craig, H. R. \& Cox (1971) and Traupel (1982). These typically examine a control volume, and then use semiempirical correlations to model the effect of the flow features inside. Although such models often capture the flow features, the link between these features and their effect on cooled turbine performance is not rigorous. They have been shown to work for certain cases, but they cannot be applied generally.

By applying an aerodynamic performance accounting method, the loss mechanisms can be examined at their source within the control-volume. Studies such as Denton (1993) and Young \& Wilcock (2002) apply exergy-based methods to examine turbomachinery flows. These methods view loss mechanisms as irreversible flow processes. The main advantage of this approach is that the different levels of design are automatically linked.

This ability to link flow features to their overall impact has been particularly useful. Bejan (1979) analysed how flow geometry might be selected to minimise the irreversibilities associated with convective heat transfer. More recently this has facilitated computational simulations to identify where the loss is being generated, such as Sciubba (1997). Herwig \& Kock (2006), Herwig (2012) and then Herwig \& Schmandt (2014) use local entropy production to evaluate heat transfer performance. Lim et al. (2011) examined the sources of irreversibility 
associated with film-cooling and compared with preliminary design models. Zhao \& Sandberg (2019) used entropy generation to assess the accuracy of different turbulence models. These irreversible processes provide a thermodynamic link between flow features and overall impact.

However, it is unclear amongst the turbomachinery community whether all irreversible flow processes are equally deleterious. An example of this is whether heat transfer between the hot mainstream and the cold coolant stream affects turbine performance. Such processes are irreversible but in many cases the performance of the gas turbine does not seem to change. Denton (1993) demonstrated this by considering the effect of cooling a turbine on its efficiency. He concluded that only the irreversibilities due to viscous processes, such as viscous dissipation, changed the performance of the turbine. Young \& Horlock (2006) illustrated the same behaviour for a simple gas turbine cycle with cooling. They also concluded that, for their simple case, the irreversibilities due to thermal processes did not change the cycle efficiency. However, they noted that this was a special case and was unlikely to be correct generally. Lim et al. (2011) state, for turbine stage, accounting for all irreversibilities associated with filmcooling would reduce efficiency by $8 \%$. They suggest a pragmatic approach is to ignore the entropy generated due to equilibrating the static temperature.

However the uncertainty concerning which irreversible flow processes change performance remains. S. M. Lim et al. (2018) apply an exergy analysis to examine the performance of a radial turbine. Uysal et al. (2018) model exergy losses to investigate the impact of cooling on gas turbine efficiency. Tailliez \& Arntz (2018) apply an exergy analysis to identify loss in turbomachines. There is no general understanding of how thermal irreversibilities affect turbine performance.

\subsection{Effect of heat transfer on boundary layer flow}

In cooled turbines, the heat transfer from the mainstream to the coolant flow creates the greatest temperature gradients in the near-wall region. This region is also characterised by large velocity gradients resulting in shear-stresses acting through the boundary layer. As heat is transferred, the temperature gradients act to change the profile of shear-stress through the boundary layer. How this aerothermal coupling affects turbine performance remains unclear. 
Many previous investigations into this coupling have focussed on the Reynolds analogy. For flows where the heat transfer is driven by forced convection, the Reynolds analogy is useful as it provides a simple and broadly applicable link between skin friction and heat transfer. The analogy works by assuming the same mechanism is responsible for transferring momentum and heat.

This aerothermal coupling has also been used to examine thermodynamic changes. Shapiro \& Hawthorne (1947) applied the Reynolds analogy to combine the effects of friction and heat transfer for steady, one-dimensional gas flows. This was to examine an effect known as aerothermopression that was hypothesised to be able achieve compression through cooling alone. The study concluded that it is impossible to raise the stagnation pressure for air by means of wall cooling alone as the benefit of cooling is always outweighed by the effect of friction.

There are many flows for which the Reynolds analogy does not hold. For turbines flows, Bons (2005) found that the Reynolds analogy continues to be a useful tool provided that additional physical mechanisms are taken into account. Most notably the freestream pressure gradient, surface roughness and freestream turbulence. Zhang et al. (2013) provide a review of attempts to produce a universal Reynolds analogy for these additional mechanisms.

The effect of cooling a boundary layer can also have a large effect on the boundary layer state. For instance, the introduction of cooling air can act to trigger turbulence while wall cooling can act to stabilise the flow. The latter is because viscosity increases with temperature for a gas, so wall cooling acts to stabilise the flow. Lees \& Lin (1946) demonstrated that complete stability cannot be achieved as higher modes appear which are not damped by increased wall cooling. However, these higher modes are only usually significant for Mach numbers greater than 3 which are rarely experienced in turbomachinery flows. For a turbine, the effect of cooling can have a large effect on the boundary layer state over the blade and consequently the performance loss. 


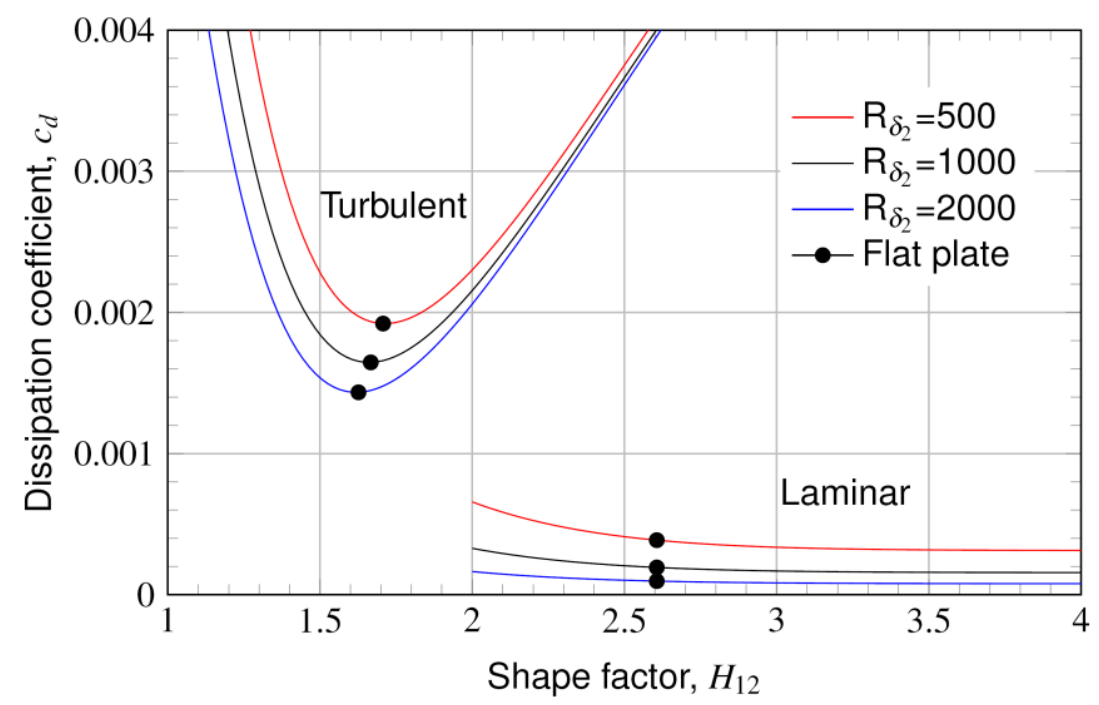

Figure 2.5 - Boundary layer dissipation coefficient for self-similar flows at a Mach number, M, of 0.7.

For a high-pressure turbine, the combination of wall cooling and a strong favourable pressure gradient near the leading edge can have a significant stabilising effect. Lin \& Sharma (2014) showed laminar flow could potentially be maintained along the suction side boundary layer for longer, (up to $R_{x} \sim 10^{8}$ ), as the high gas-to-wall temperature ratio provided a stabilising effect.

Changes in turbine performance due to flow processes in the boundary layer have been modelled using the dissipation coefficient. Denton (1993) used the dissipation coefficient to model the boundary layer loss mechanisms, noting that it is relatively constant for the turbulent flow found in gas turbines $\left(c_{d} \approx 0.002\right)$. Although this is a useful assumption, the dissipation coefficient can vary substantially depending on transition, turbulence and boundary layer state.

For integral boundary layer methods, the dissipation coefficient is often modelled to better calculate skin friction. These models predict the dissipation coefficient for a family of boundary layer profiles. For instance, Drela \& Gilest (1986) applied a $G$ - $\beta$ locus and assumed a separation of scales to define a family of profiles for the inner and outer region. Figure 2.5 shows how this model for dissipation coefficient in laminar and turbulent flow varies with shape factor and Reynolds numbers. This clearly demonstrates that the state of the boundary layer has a very large effect on viscous dissipation. 
The effect of heat transfer on dissipation coefficient has been investigated by Walz (1967) to improve integral boundary layer methods. Walz applied a scaling argument to suggest the dissipation coefficient responded to heat transfer in the same way as the skin friction coefficient. Beyond this, little is understood about how the dissipation coefficient varies with heat transfer.

More studies have examined skin friction, but they tend to focus on adiabatic, compressible flow. Even here, some fundamental questions remain. For instance, Wenzel et al. (2018) state that no generally accepted compressible mean velocity profile exists for turbulent flow. In addition, it is still unclear by which Reynolds number the wake region in a compressible turbulent boundary layer can be scaled. Goyne et al. (2003) provide experimental measurements, but the analytical nature of compressibility on the skin friction coefficient is still unknown.

In summary, the aerothermal coupling between heat transfer and shear-stresses is typically described by the Reynolds analogy. The Reynolds analogy remains a useful way of examining turbine flow. The effect of the boundary layer on turbine performance can be modelled using a dissipation coefficient. It has been suggested that heat transfer affects skin friction and dissipation coefficient in a similar way. However, it is unclear how heat transfer affects dissipation coefficient.

\subsection{Practical trade-offs in cooled turbine blade design}

Designing a turbine is an intricate multidisciplinary task. The final design is a compromise between aerothermal performance and constraints arising from mechanical, structural or material requirements. Here, the challenge has been simplified. This section will focus on the effect of cooling a turbine and will examine the main concerns at two different design levels.

When cooling at the cycle level, the principle trade is between permitting a higher operating temperature, which raises efficiency, and more cooling air bypassing the combustor, which reduces efficiency. This balance has been considered studies such as Horlock (2001). Typically, their goal is to relate the increase in coolant mass flow to the decrease in efficiency. 
This can then be used in studies, such as Wilcock, Young, \& Horlock (2005), to find an optimal operating temperature. Advances in cooling technologies at the cycle level act to reduce the cost of using additional coolant mass flow to the cycle.

When cooling at the component level, the principle balance is between ensuring enough cooling is used so that the component is cooled sufficiently to survive during operation and reducing the amount of cooling to ensure that it is as efficient as possible. This balance has been considered by studies such as Horlock and Torbidoni (2006). Typically, the goal of these studies is to develop cooling technologies with a greater cooling effectiveness. By increasing cooling effectiveness, less mass flow is required to achieve the same level of cooling. This reduction in required mass flow changes the balance of cooling at the cycle level.

This exchange between the effect of cooling at the component and cycle level is an iterative process as there is not currently a link between the two levels of design. By better understanding how changes in design impact efficiency, a more informed balance between aerothermal performance and design constraints may be achieved.

\subsection{Research questions}

From the literature, five research questions have been raised. These will be answered in the following chapters.

\section{How should cooled turbine performance be defined?}

Currently, there is no clear agreement about how cooled turbine performance should be defined. This is because of the difficulty in defining ideal work when cooling is introduced. Chapter 4 seeks to identify an aerodynamic performance accounting method that both links the different levels of design and agrees with industrial experience.

\section{What effect does heat transfer have on turbine loss mechanisms?}

The flow features responsible for changing the efficiency of a cooled turbine are currently uncertain. In particular, it is unknown what effect heat transfer across temperature differences 
has on turbine performance. In Chapter 5, the effect of heat transfer on turbine loss mechanisms is demonstrated.

3. How can the effect of heat transfer on these loss mechanisms be modelled in design?

When calculating the performance of a cooled turbine, the effect of heat transfer is currently neglected. Chapter 6 aims to both present how loss models can be updated and demonstrate how the performance can be compared at two different levels of design.

\section{How does heat transfer affect viscous dissipation in the boundary layer?}

In a cooled turbine, the near-wall temperature gradients act to change the profile of shear-stress through the boundary layer. These shear-stresses are responsible for viscous dissipation, which acts to reduce turbine performance. It is currently unclear whether the near-wall temperature gradient acts to change the dissipation coefficient. This will be investigated in Chapter 7 .

5. What are the implications for cooled turbine blade design?

The practical design of a cooled turbine is a balance between many competing objectives. In Chapter 8 , the effect of cooling on performance at both the cycle level and the component level will be considered. 


\section{Chapter 3 Methodology}

In this thesis, the effect of heat transfer on turbine performance has been numerically investigated by examining turbine cascades and boundary layers. The turbine cascades were simulated using ANSYS Fluent while the boundary layer cases were simulated using a compressible Blasius solution, ANSYS Fluent, TEXSTAN and 3DNS. This chapter is split into two sections providing details of the numerical methods applied to turbine cascades and boundary layers respectively.

\subsection{Turbine cascade simulations}

The turbine cascade results presented in Chapter 5 and Chapter 6 analyse an internally-cooled and a film-cooled high-pressure turbine blade respectively.

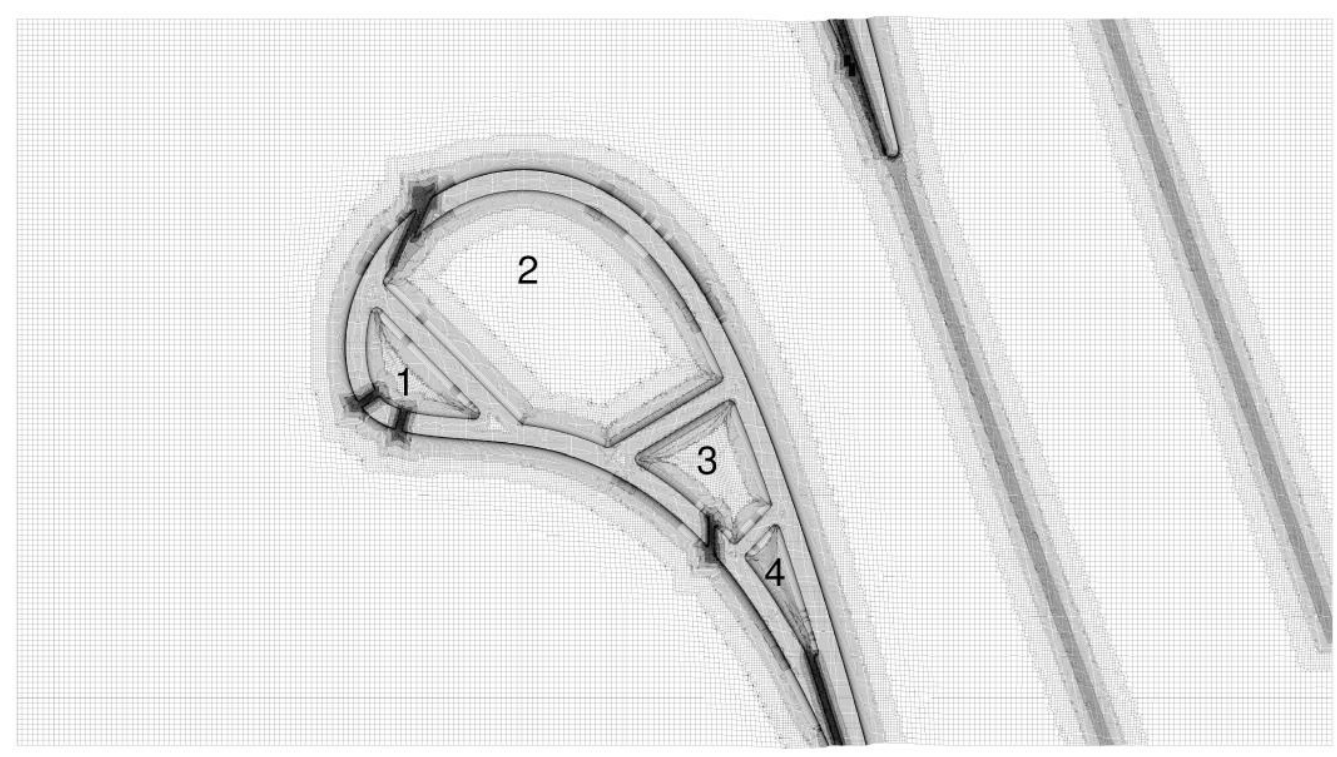

Figure 3.1 - Mesh for simulating a film-cooled high-pressure turbine blade. 
Table 3.1 - Summary of mesh sizes used for simulating internally-cooled and film-cooled turbine blades.

\begin{tabular}{lcc}
\hline & Internally-cooled & Film-cooled \\
\hline Number of cells & $7.78 \times 10^{6}$ & $15.11 \times 10^{6}$ \\
Number of faces & $22.59 \times 10^{6}$ & $40.84 \times 10^{6}$ \\
Number of nodes & $6.98 \times 10^{6}$ & $10.50 \times 10^{6}$ \\
$1^{\text {st }}$ cell $y^{+}$ & $\sim 1$ & $\sim 1$ \\
\hline \hline
\end{tabular}

Both cases have been taken from a mid-height $1.5 \%$ section of the span and share the same boundary conditions and blade profile. The overall domain is adiabatic, but with heat transfer within the conjugate CFD domain between the mainstream and coolant.

The turbine cascades were meshed using BOXER and solved using ANSYS Fluent 17.0. The film-cooled mesh is shown in Figure 3.1. A summary of the mesh sizes used for the internal and film cooled blade are provided in Table 3.1. A summary of the CFD is provided in Table 3.3. Finally, a summary of the flow conditions is provided in Table 3.2. The main difference between the internal and film cooled blades is the amount of mesh required to resolve the cooling holes.

Table 3.2 - Summary of flow setup for all turbine simulations, unless otherwise stated.

\begin{tabular}{lccc}
\hline & Flow conditions & Flow conditions \\
\hline $\mathrm{R}_{\text {in }}$ & $3.5 \times 10^{5}$ & $\dot{m}_{c, 1} / \dot{m}_{\text {in }}$ & $1 \%$ \\
$\mathrm{M}_{\text {in }}$ & 0.255 & $\dot{m}_{c, 2} / \dot{m}_{\text {in }}$ & $1 \%$ \\
$\mathrm{M}_{\text {out }}$ & 0.85 & $\dot{m}_{c, 3} / \dot{m}_{\text {in }}$ & $0.5 \%$ \\
Inlet angle & $-45^{\circ}$ & $\dot{m}_{c, 4} / \dot{m}_{\text {in }}$ & $0.5 \%$ \\
Outlet angle & $72^{\circ}$ & $\mathrm{M}_{c}$ & 0.1 \\
$T_{t, \text { in }}$ & $2000 \mathrm{~K}$ & $T_{t, c}$ & $1000-2000 \mathrm{~K}$ \\
\hline \hline
\end{tabular}


Table 3.3 - Summary of CFD setup for all turbine blade simulations, unless otherwise stated.

\begin{tabular}{ll}
\hline \hline & CFD setup \\
\hline RANS model & Spalart \& Allmaras (1992) \\
Viscosity model & Sutherland's law (Sutherland 1893) \\
Thermal conductivity (fluid) & Kinetic theory \\
Thermal conductivity (solid) & Polynomial (Anderson, Patwa, \& Shin 2006) \\
Specific heat, $c_{p}$ & 1006 J / (kg K) \\
Pressure-velocity coupling & Coupled \\
Gradient discretisation & Green-Gauss cell-based \\
Spatial discretisation & MUSCL \\
\hline \hline
\end{tabular}

All simulations presented in this thesis have analysed a perfect gas. This was chosen to enable the physical mechanisms to be presented with clarity.

Mesh sensitivity studies for both the internally-cooled and film-cooled cases are shown in Figure 3.2. The red, blue and black lines show the error in the change of exergy and euergy loss coefficient (from inlet to outlet) and in the area-averaged heat transfer coefficient (HTC).
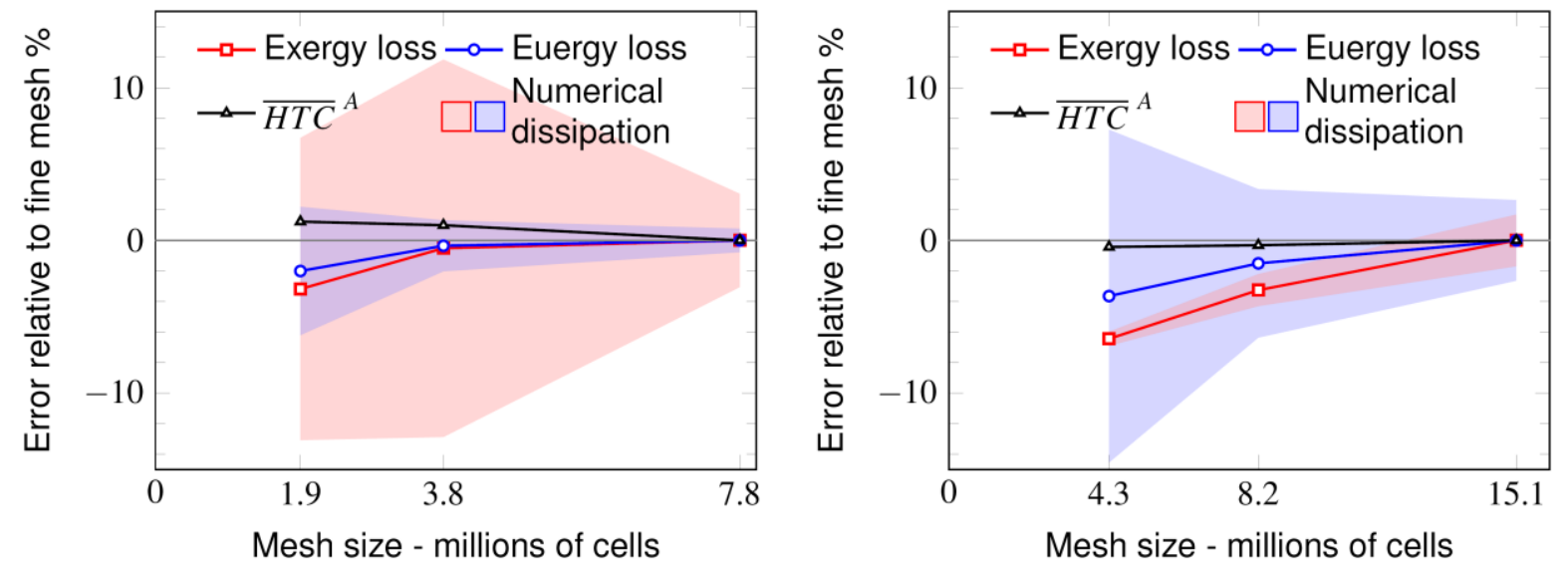

Figure 3.2 - Mesh independence study of internally-cooled (left) and film-cooled (right) CFD cases. 
The results illustrate that all the metrics are mesh independent within $1.0 \%$ for the internallycooled case and within $3.3 \%$ for the film-cooled case.

A central part of this work has been balancing the source and sink terms within the blade row to the flux terms into and out of the blade row. Herwig \& Kock (2007) referred to these as indirect and direct methods. This balance can also be examined for each cell as a metric of mesh quality. The overall balance is shown by the shaded regions in Figure 3.2 and the transport equations for the exergy and euergy method have been presented by Miller (2013). This balance is challenging to achieve numerically as the source terms have a quadratic dependence on the velocity and temperature gradients. This challenge resulted in Zlatinov et al. (2012) using the source terms as a qualitative measure. To resolve these, high grid densities and higher order methods are required. For the internally-cooled case, the exergy-balance is within $3.1 \%$ and the euergy balance is within $0.8 \%$. For the film-cooled case, the exergybalance is within $1.7 \%$ and the euergy balance is within $2.7 \%$. To reduce the error would require a computational technique that captured more of the real physical process, such as LES or DNS.

\subsection{Boundary layer simulations}

The boundary layer flow in Chapter 7 was examined using a compressible Blasius solution, TEXSTAN RANS models, ANSYS Fluent RANS models and high-fidelity methods. These will be examined in turn. However, the flow conditions are similar for all data presented.

As shown in Figure 3.3, a plane within each simulation has been examined. The plane has a mainstream Mach number of 0.7 and a Reynolds number based upon momentum thickness of 400 for laminar flow or 1000 for turbulent flow.
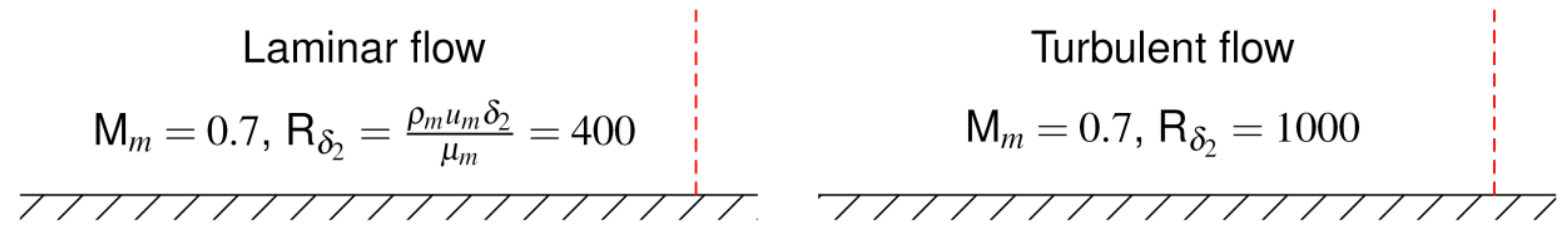

Figure 3.3 - Laminar and turbulent flow evaluated at $\mathrm{R}_{\delta_{2}}=400$ and $\mathrm{R}_{\delta_{2}}=1000$ respectively. 
Table 3.4 - Summary of laminar and turbulent boundary layer flow conditions.

\begin{tabular}{lcc}
\hline \hline & Laminar flow & Turbulent flow \\
\hline Inlet total temperature, $T_{t, \text { in }}$ & $1500 \mathrm{~K}$ & $1500 \mathrm{~K}$ \\
Mach number, $\mathrm{M}$ & 0.7 & 0.7 \\
Specific heat, $c_{p}$ & $1005 \mathrm{~J} /(\mathrm{kg} \mathrm{K})$ & $1005 \mathrm{~J} /(\mathrm{kg} \mathrm{K})$ \\
Prandtl number, $\mathrm{P}$ & 0.72 & 0.72 \\
Reynolds number, $\mathrm{R}_{\delta_{2}}$ & 400 & 1000 \\
Inlet turbulence intensity, $\mathrm{T}_{\text {in }}$ & - & $5 \%$ \\
\hline \hline
\end{tabular}

A summary of the CFD setup is shown in Table 3.4. These conditions have been chosen to investigate the suction-side boundary layer of a high-pressure turbine blade. The main differences between laminar and turbulent flow are the Reynolds number at which the flow is evaluated and the inlet turbulence intensity. This is $5 \%$ for turbulent flow.

\subsubsection{Compressible Blasius solution}

For a laminar boundary layer, the energy equation is coupled to the momentum equation through viscosity. To analytically solve the compressible Blasius solution, these two equations need to be decoupled. This can be achieved by assuming a linear temperature-viscosity relationship as shown in Equation 3.1.

$$
\frac{\mu}{\mu_{m}}=C_{w} \frac{T}{T_{m}}
$$

The constant of proportionality, $C_{w}$, is the Chapman-Rubesin parameter. The effect of this constant is shown on the left-hand side of Figure 3.4. 

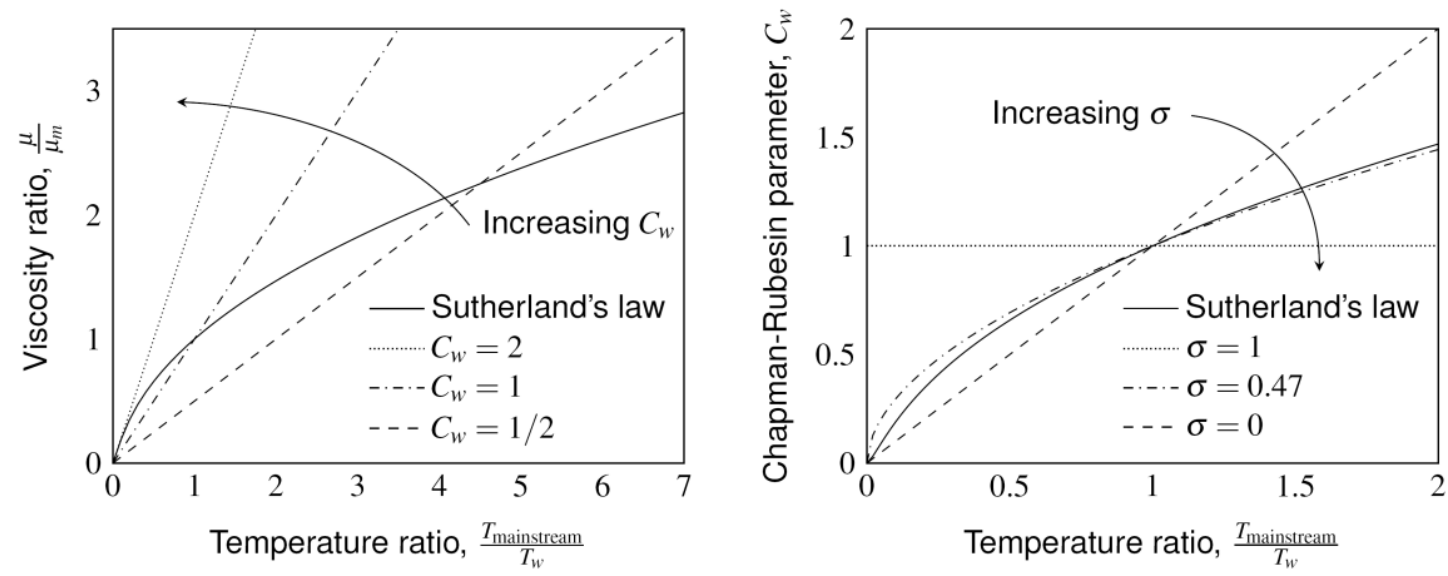

Figure 3.4 - Compressible Blasius solution viscosity ratio (left) and Chapman-Rubesin parameter, $C_{w}$, (right) variation with mainstream-to-wall temperature ratio,

The Chapman-Rubesin parameter was chosen to match the viscosity at the wall. However, to best match the wall viscosity as the wall temperature varies, the Chapman-Rubesin parameter should vary with the mainstream-to-coolant temperature ratio. This is approximated in Equation 3.2 and shown on the right-hand side of Figure 3.4.

$$
C_{w}=\frac{\mu_{w}}{\mu_{m}} \frac{\rho_{w}}{\rho_{m}} \approx\left(\frac{T_{m}}{T_{w}}\right)^{1-\sigma}
$$

To numerically solve the Blasius equation, a Newton-Raphson method was developed. The derivation of the compressible Blasius method is discussed by Anderson (1989) and extended in Appendix C.2 to examine dissipation.

\subsubsection{RANS simulations (ANSYS Fluent)}

The boundary layer RANS simulations in ANSYS Fluent 17.0 were meshed using ANSYS ICEM CFD 16.0. These simulations were two-dimensional and applied a mesh of 1.5 million cells.

The $1^{\text {st }}$ cell height for the different mainstream-to-wall temperature ratios investigated are provided in Table 3.5. These are all significantly below unity. By lowering the wall temperature, the $1^{\text {st }}$ cell $y^{+}$increased. 
Table 3.5 - Summary of $1^{\text {st }}$ cell $y^{+}$at $\mathrm{R}_{\delta_{2}}=1000$ used for ANSYS Fluent boundary layer simulations.

\begin{tabular}{cccccc}
\hline \hline$\Omega=\frac{T_{m}}{T_{w}}$ & 0.92 & 1.09 & 1.37 & 1.82 & 2.73 \\
\hline $1^{\text {st }}$ cell $y^{+}$ & 0.05 & 0.06 & 0.08 & 0.12 & 0.20
\end{tabular}

A summary of the CFD setup is provided in Table 3.6. A small inlet velocity and temperature profile, corresponding to $\mathrm{R}_{\delta_{2}} \approx 50$, was used to avoid leading-edge errors.

Three turbulence models have been applied in this analysis. The first is the S-A model presented by Spalart \& Allmaras (1992). The second is the standard $k$ - $\omega$ model presented by Wilcox (1988). The third is the standard $k-\epsilon$ model presented by Launder \& Spalding (1974).

Figure 3.5 shows the uncertainty in dissipation coefficient with mainstream-to-wall temperature ratio for these boundary layer simulations. This uncertainty is based upon the imbalance between the local volumetric terms and the flux terms in the euergy equation. The imbalance occurs due to numerical dissipation and, for all the boundary layer simulations, is less than $1 \%$.

Table 3.6 - Summary of ANSYS Fluent setup used for boundary layer simulations.

\begin{tabular}{lc}
\hline \hline & ANSYS Fluent setup \\
\hline Inlet velocity and temperature profiles & Turbulent \\
Viscosity model & Sutherland's law (Sutherland 1893) \\
Thermal conductivity & Kinetic theory \\
Pressure-velocity coupling & Coupled \\
Gradient discretisation & Green-Gauss node-based \\
Spatial discretisation & MUSCL \\
\hline \hline
\end{tabular}




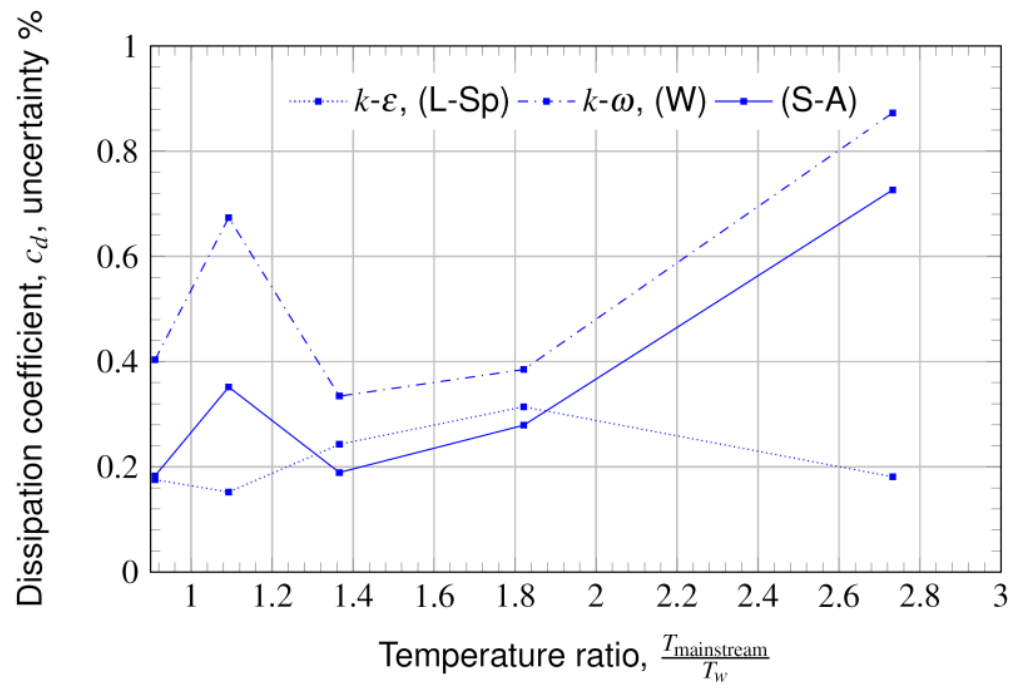

Figure 3.5 - Uncertainty in dissipation coefficient due to the imbalance of the euergy equation in ANSYS Fluent simulations.

\subsubsection{RANS simulations (TEXSTAN)}

TEXSTAN (Crawford 1985) is a finite-difference solver for two-dimensional boundary layer flow developed from STAN5 (Crawford \& Kays 1976). It has been formulated to solve equations for heat, mass and momentum transfer, includes turbulence models to provide mean field closure via the eddy viscosity model and provides turbulent heat flux closure via a turbulent Prandtl number. TEXSTAN was chosen to provide an additional assessment of flat plate boundary layers and to complement Fluent simulations.

The setup used for the TEXSTAN simulations is provided in Table 3.7. This setup is the same as the ANSYS Fluent simulations presented in the previous section, but with a different solver and turbulence models.

Four turbulence models in TEXSTAN have been used in this thesis. These cover three levels of mean field closure with the eddy viscosity model. These are the mixing-length model (M-L), the one equation model of Hassid \& Poreh (1975) and the two equation models of Launder \& Sharma (1974) and Jones \& Launder (1973). 
Table 3.7 - Summary of TEXSTAN setup used for boundary layer simulations.

\begin{tabular}{lc}
\hline \hline & TEXSTAN setup \\
\hline Inlet velocity and temperature profiles & Turbulent (kstart =3) \\
Viscosity model & Sutherland's law (kfluid = 14) \\
Energy equation turbulence model & Variable turbulent Prandtl number (ktme = 3) \\
Mixing-length model (M-L) & $\quad$ Prandtl mixing length with Van Driest damping \\
One-equation model, $k,(\mathrm{H}-\mathrm{P})$ & Hassid \& Poreh $(1975)(\mathrm{ktmu}=11)$ \\
Two-equation model, $k-\epsilon,(\mathrm{L}-\mathrm{Sh})$ & Launder \& Sharma (1974) $(\mathrm{ktmu}=21)$ \\
Two-equation model, $k-\epsilon,(\mathrm{J}-\mathrm{L})$ & Jones \& Launder $(1973)(\mathrm{ktmu}=24)$ \\
\hline \hline
\end{tabular}
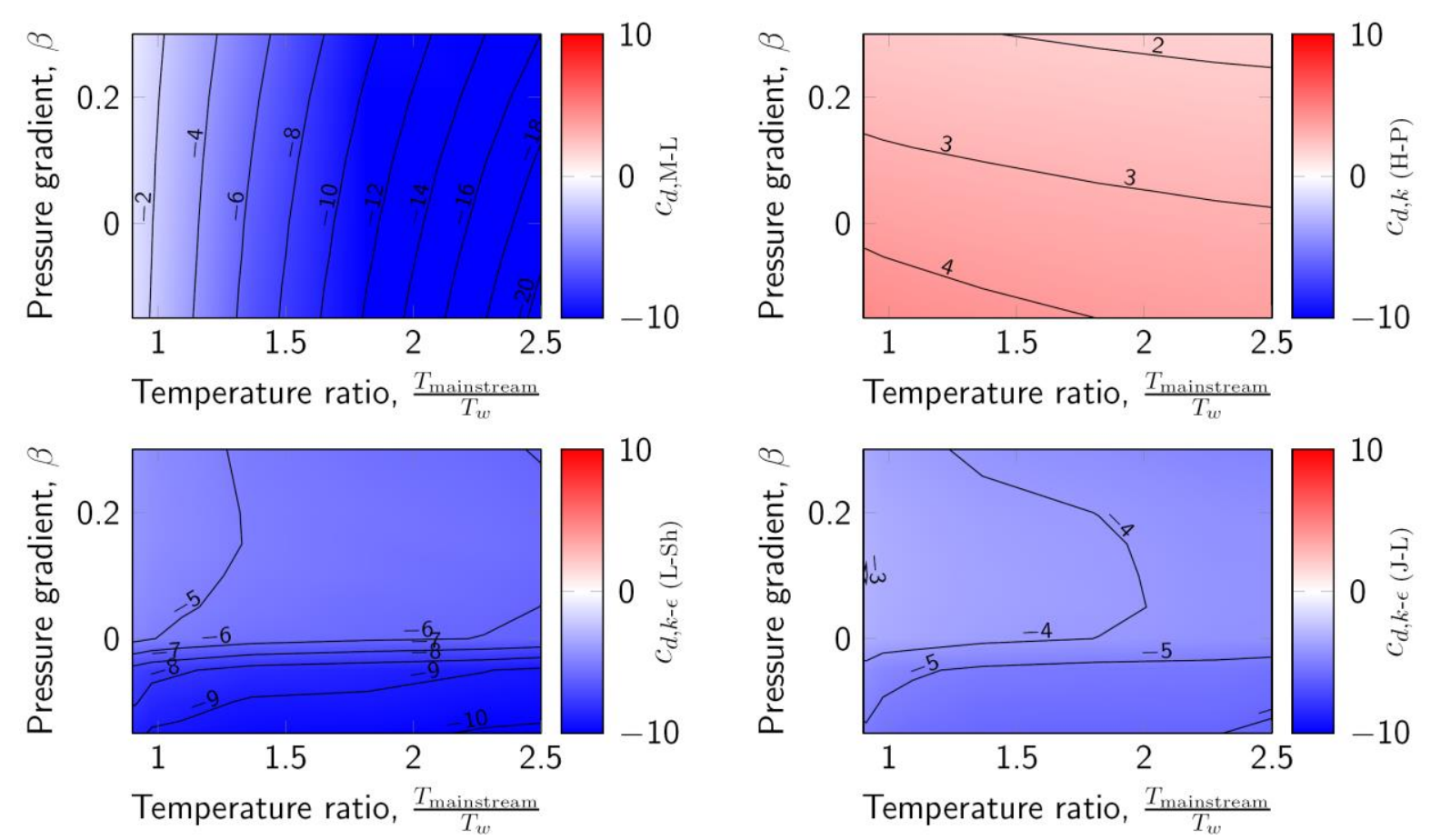

Figure 3.6 - Uncertainty in dissipation coefficient due to the imbalance of the euergy equation in TEXSTAN simulations. 
The uncertainty in the dissipation coefficient can be estimated for the TEXSTAN simulations as well. The change in dissipation coefficient due to the imbalance in the euergy equation is shown in Figure 3.6. The mixing-length model (top-left) struggles to achieve a good balance away from the adiabatic case. The $k-\epsilon$ (L-Sh) model (bottom-left) has difficulty in balancing the euergy equation in adverse pressure gradients. Otherwise, the models all achieve a good balance and can assess the dissipation coefficient with confidence.

\subsubsection{High-fidelity simulations (3DNS)}

The nature of aerothermal coupling in a boundary layer was examined using a solver called 3DNS. This is a compressible finite difference Navier-Stokes solver for use on structured multi-block meshes and was first presented by Wheeler, Dickens, \& Miller (2018).

One of the meshes used for this study is shown in Figure 3.7. Characteristic boundary conditions were applied at the inlet and outlet with a sponge zone to minimize reflections. The cells in these sponge zones grew with an expansion ratio, $\alpha$, of 1.1 parallel to the $\mathrm{x}$ direction. The cells grew with an expansion ratio around 1.04 in the y direction between the bottom and top walls. The top surface was a slip wall as this was found to be much more stable with high freestream turbulence intensity. To minimise the pressure-gradient, this top surface was designed to grow with the boundary layer displacement thickness, $\delta_{1}$. The boundary condition for the bottom surface was an isothermal wall.

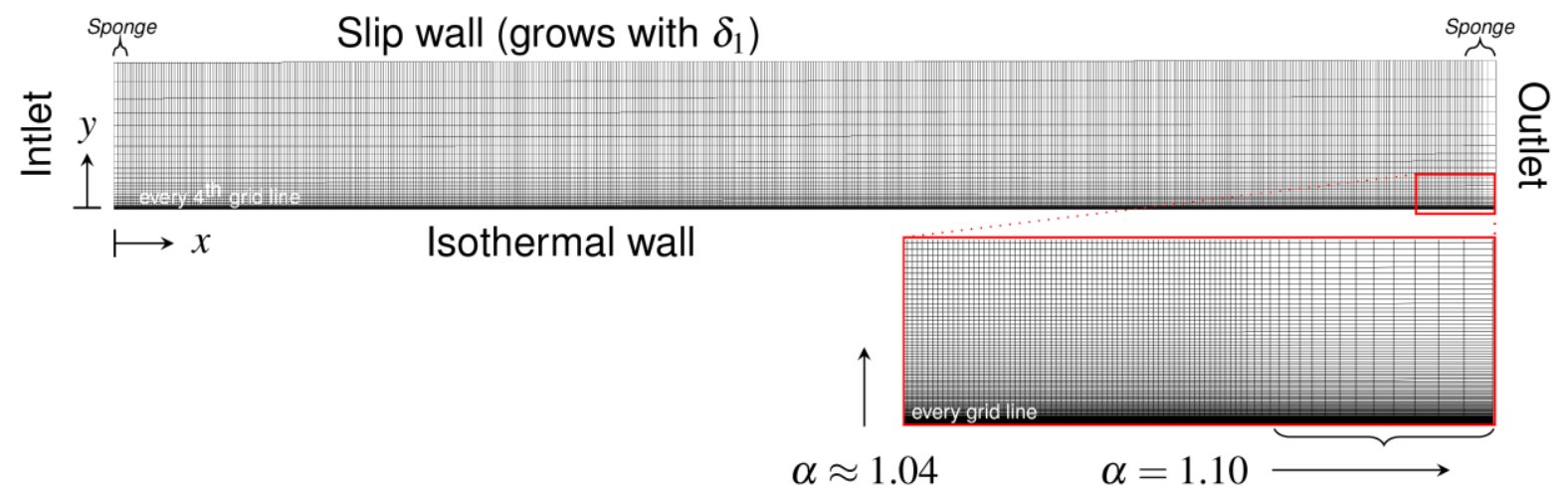

Figure 3.7 - Mesh setup used to simulate flat plate boundary layer using 3DNS. 
Table 3.8 - Summary of $1^{\text {st }}$ cell $y^{+}$at $R_{\delta_{2}}=1000$ used for 3DNS boundary layer simulations

\begin{tabular}{cccc}
\hline \hline$\Omega=\frac{T_{m}}{T_{w}}$ & $x^{+}$ & $1^{\text {st }}$ cell $y^{+}$ & $z^{+}$ \\
\hline 0.92 & 10.89 & 0.77 & 7.57 \\
1.19 & 14.34 & 1.01 & 10.12 \\
1.38 & 17.24 & 1.19 & 11.99 \\
1.61 & 20.44 & 1.41 & 14.58 \\
1.83 & 23.69 & 1.58 & 16.47 \\
\hline
\end{tabular}

The dimensions of the mesh for the boundary layer simulations were $2400 \times 144 \times 576$ in the $\mathrm{x}, \mathrm{y}$ and $\mathrm{z}$ directions respectively. This 200 million cell mesh was solved on ARCHER. At $R_{\delta_{2}}=1000$, the dimensions of the $1^{\text {st }}$ cell closest to the wall are shown in Table 3.8. These simulations will be referred to as DNS as no subscale modelling was applied. The resolution is comparable to similar simulations presented by Li et al. (2009) and $\mathrm{Wu}$ and Moin (2009), however they represented the temperature field using a passive scalar. The resolution is low compared to simulations that recycle the boundary layer. Wenzel et al. (2018) provides a summary of flat plate DNS for comparison.

The uncertainty in dissipation coefficient due to the difference between production of turbulent kinetic energy and the dissipation of turbulent kinetic energy is shown in Table 3.9. For an equilibrium turbulent boundary layer, the difference should be zero. The difference arises because the mesh resolution is not sufficient to directly capture all of the dissipative scales. These values were measured at $\mathrm{R}_{\delta_{2}} \approx 1000$.

Table 3.9 - Uncertainty in dissipation coefficient due to the imbalance between production and dissipation of turbulent kinetic energy in 3DNS.

\begin{tabular}{cccccc}
\hline \hline$\Omega=\frac{T_{m}}{T_{w}}$ & 0.92 & 1.19 & 1.38 & 1.61 & 1.83 \\
\hline$c_{d}$ uncertainty & $7.35 \%$ & $6.34 \%$ & $7.09 \%$ & $6.91 \%$ & $11.37 \%$ \\
\hline \hline
\end{tabular}




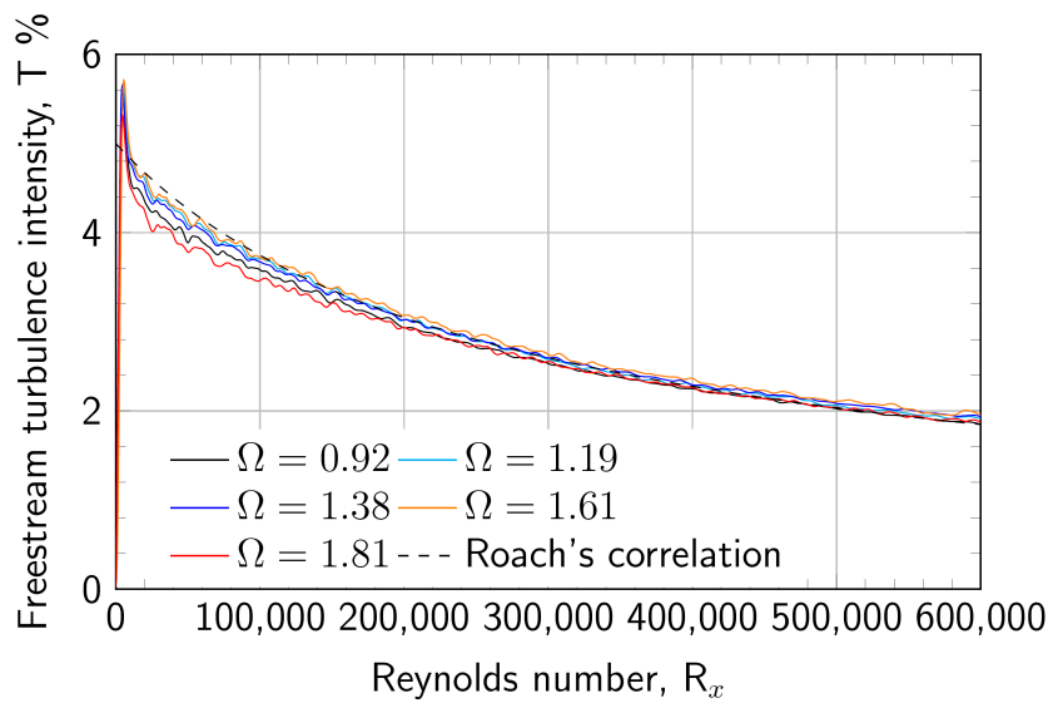

Figure 3.8 - Decay of freestream turbulence intensity, T, for 3DNS simulations.

An inlet velocity and temperature profile, corresponding to $R_{\delta_{2}} \approx 50$, was introduced to avoid non-physical leading edge effects. Wu (2017) provides a summary of inflow turbulence generation methods. Here, the full boundary layer evolution as simulated. Simulating transition was more computationally expensive than techniques, such as a recycling method. This method was chosen to allow the viscous and thermal boundary layers to grow physically, rather prescribing the relationship between them. This was particularly important when investigating the effect of aerothermal coupling. 
Table 3.10 - Summary of the 3DNS setup used for boundary layer simulations.

\begin{tabular}{ll}
\hline \hline & 3DNS setup \\
\hline Viscosity model & Sutherland's law \\
Time stepping & Explicit 4 step Runge-Kutta scheme. \\
Filter & $8^{\text {th }}$ order \\
Characteristic boundary conditions & Poinsot \& Lelef (1992) \\
Inflow turbulence & Phillips \& Fyfe (2011) \\
Convective fluxes & Skew-split formulation \\
Spatial discretisation & $\begin{array}{l}\text { th } \text { order dispersion relation preserving finite } \\
\text { difference scheme, Tam \& Webb (1993) }\end{array}$ \\
\hline \hline
\end{tabular}

Figure 3.8 shows the decay of freestream turbulence intensity along the length of the simulation. The turbulence intensity is very similar for all of the DNS cases and agrees with the correlation proposed by Roach \& Brierley (1990).

As the top boundary condition is a solid interface, a perfectly zero pressure gradient could not be achieved. A solid interface was used due to the freestream turbulence. Figure 3.9

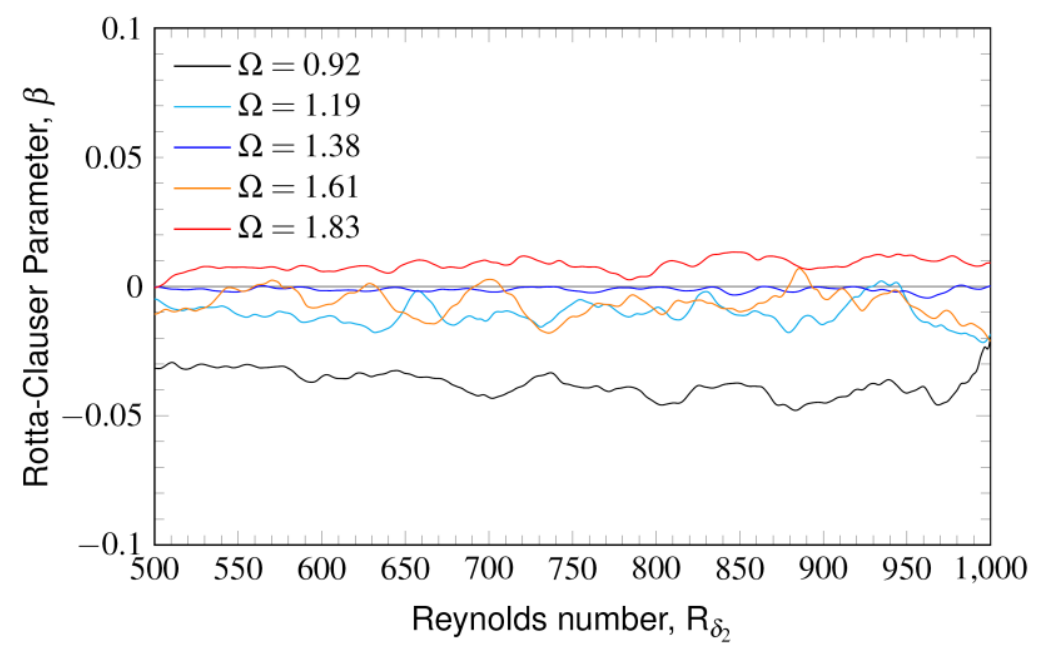

Figure 3.9 - Flat plate pressure gradient in 3DNS simulations. 
shows the pressure gradients for the flat plate simulations. They are all near-zero and relatively constant long the length of the plate. Further details regarding the setup for the 3DNS are provided in Table 3.10.

\subsection{Summary}

For the turbine cascades and boundary layer simulations presented in this thesis, a good agreement between the flow processes and their effect on the overall control volume has been obtained. This has been achieved using high mesh densities to thoroughly resolve the flow. When investigating these boundary layers, a range of different numerical codes and turbulence modelling techniques have been applied to assess the confidence with which a designer can model the effect of heat transfer on turbine performance. 


\section{Chapter 4 Defining cooled turbine performance}

No consensus exists for defining the efficiency of a cooled turbine. More importantly, no systematic method of loss accounting exists which allows the losses of individual components within the turbine stage (e.g. a rim seal loss or a cooling hole loss) to be related to the overall stage efficiency. This limitation is shown in Figure 4.1 where the impact of a local region of heat transfer on the overall loss coefficient of a turbine cascade is unknown. This chapter aims to compare aerodynamic performance accounting methods, identify their key differences and demonstrate their applicability to cooled turbines. There are three sections:

First, two general methods are examined. These methods provide a thermodynamic framework to link performance across all scales of design. This section compares the two general methods currently available; exergy and euergy.

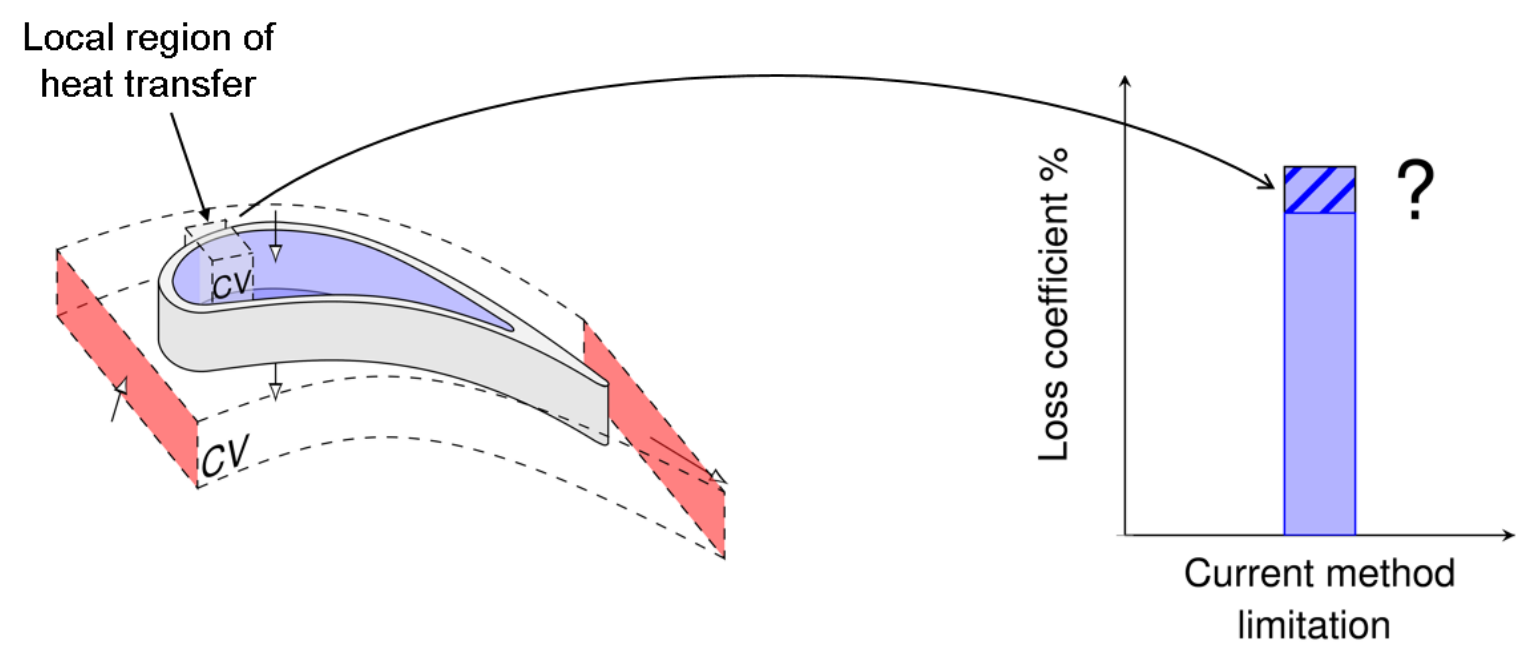

Figure 4.1 - A cooled turbine loss coefficient can be calculated from the overall control volume. Currently, the contribution from a sub-control volume cannot be calculated. 
Second, two industrially-focussed performance accounting methods are examined. The euergy method and power-balance method have been derived to reflect the performance of different applications. As the euergy method has been derived for turbomachinery, it is expected that it is better suited for examining cooled turbines. However, the power-balance method can be generalised and it's the applicability is unclear.

Finally, three traditional turbine loss coefficients are examined. These loss coefficients have been derived to reflect the performance of uncooled turbines, but it is not clear whether they extend to cooled turbines or how to attribute loss to regions of the flow. This section examines extending the enthalpy, entropy and stagnation pressure loss coefficients using these generalised methods.

\subsection{Comparing general methods (exergy and euergy)}

The definition of a general method considers a system of interest, a reference environment and processes acting between them that obey the laws of thermodynamics. This is powerful because a general method can be applied across a wide range of systems and at any scale. The general methods aim to use a set of processes to extract the maximum (ideal) work from the combined system and environment. This section will compare two such methods; exergy and euergy.

The exergy method views the ideal work as the work that could be extracted by a universal reversible machine shown in Figure 4.2. The universal reversible machine sets the processes that can act between the system and environment. This machine brings the flow into

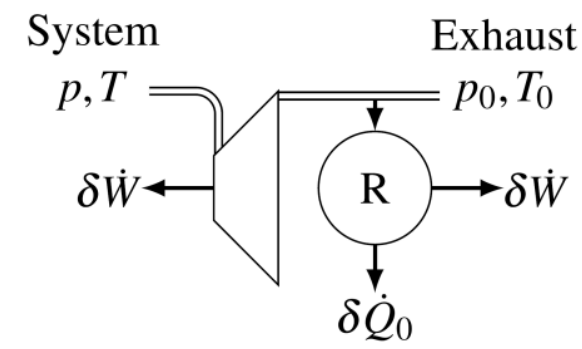

Exergy method

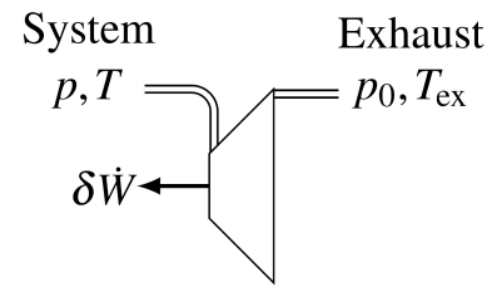

Euergy method

Figure 4.2 - Devices required to extract ideal work for the exergy method (left) and euergy method (right). 
mechanical equilibrium (same pressure) and thermal equilibrium (same temperature) with the reference environment. To achieve this ideal work, the universal reversible machine could be made up of a reversible adiabatic turbine, to bring the flow into mechanical equilibrium, and a reversible heat engine, to bring the flow into thermal equilibrium.

The euergy method views the ideal work as the work that can be extracted by a reversible adiabatic turbine shown in Figure 4.2. This turbine can only bring the flow into mechanical equilibrium (same pressure) so the exhaust temperature differs from the reference environment. Although the exhaust still has work potential, it cannot be extract by the thermodynamic processes available to a turbine, so it is considered to be lost.

The exergy and euergy methods aspire to different devices. These devices operate between the system and the reference environment acting to constrain heat and work interactions. This constraint stems from the second law of thermodynamics which specifies that a heat engine can only convert a fraction of heat into work demonstrated by Jardine \& Miller (2019). The ideal work of a device depends on the amount of work that can be extracted from a heat addition.

This value of heat relative to work is shown in Figure 4.3 where a small heat addition, $\delta \dot{Q}$, is transferred to the flow of a perfect gas. The heat addition changes the maximum amount of work that could be extracted from the flow.

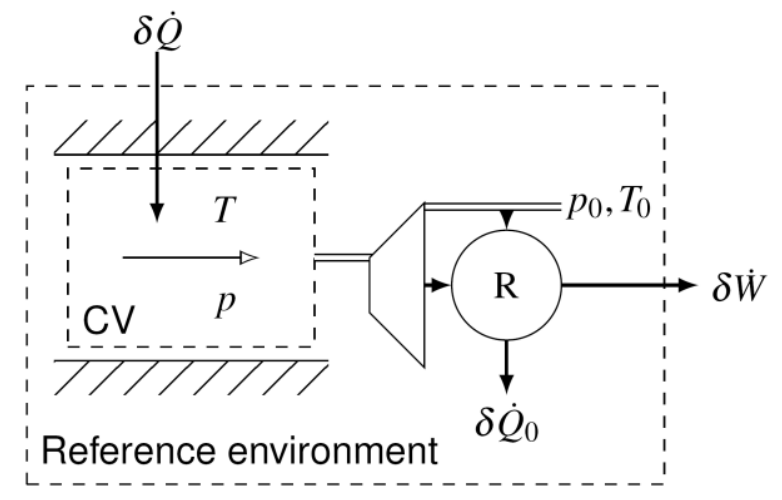

(a)

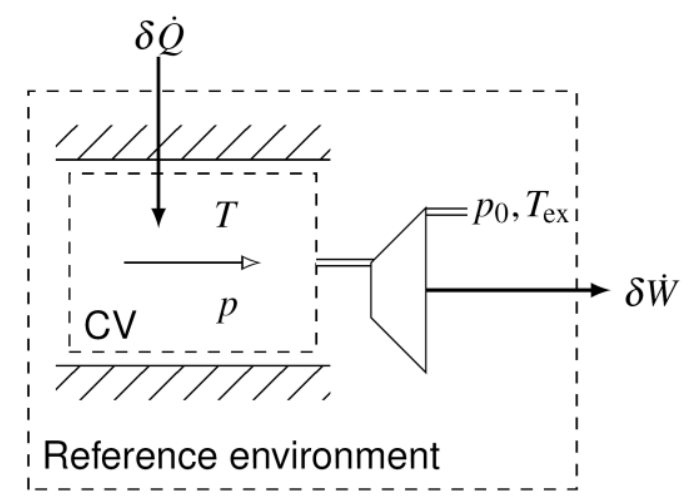

(b)

Figure 4.3 - Value of heat for the exergy method (a) and euergy method (b). 
For the exergy method, the increase in ideal work is the increase in work that could be extracted by a universal reversible machine exhausting to an environmental pressure, $p_{0}$, and an environmental temperature, $T_{0}$. This sets the value placed on heat to be the local Carnot cycle efficiency, $\eta_{t h}^{\mathrm{e}}$, shown in Equation 4.1.

$$
\delta \dot{W}=\underbrace{\left[1-\frac{T_{0}}{T}\right]}_{\eta_{t h}^{\mathrm{e}}} \delta \dot{Q}
$$

For the euergy method, the increase in ideal work is the increase in work that could be extracted by a reversible adiabatic turbine alone exhausting to an environmental pressure, $p_{0}$. The exhaust temperature, $T_{e x}$, varies from the environment as the heat and work interactions are constrained to use a turbine. This sets the value placed on heat to be the local Joule (Brayton) cycle efficiency, $\eta_{t h}^{m}$, shown in Equation 4.2.

$$
\delta \dot{W}=\underbrace{\left[1-\left(\frac{p_{0}}{p}\right)^{\frac{\gamma-1}{\gamma}}\right]}_{\eta_{t h}^{\mathrm{m}}} \delta \dot{Q}
$$

To examine the implications this difference, consider the loss coefficient of a stationary adiabatic blade row which is defined as:

$$
\omega=\frac{\sum_{\text {in }} \dot{W}_{\text {ideal }}-\sum_{\text {out }} \dot{W}_{\text {ideal }}}{\sum_{\text {out }} \dot{K} E}
$$

where $\dot{W}_{\text {ideal }}$ is the maximum work that can be extracted from each stream and the summation is over all the mainstream and coolant streams.

If the exergy method is applied, the ideal work in Equation 4.3 is defined as the work that can be extracted by a universal reversible machine and is calculated at all of the inlets and outlets as shown in Figure 4.4. For this process, the thermal efficiency is set by the Carnot cycle efficiency, $\eta_{t h}^{\mathbf{e}}$, resulting in the ideal flow work to become the flow exergy, $\mathbf{e}_{\mathbf{f}}$. For a steady flow, the exergy loss coefficient, $\omega^{\mathbf{e}}$, is defined by Equation 4.4 . 


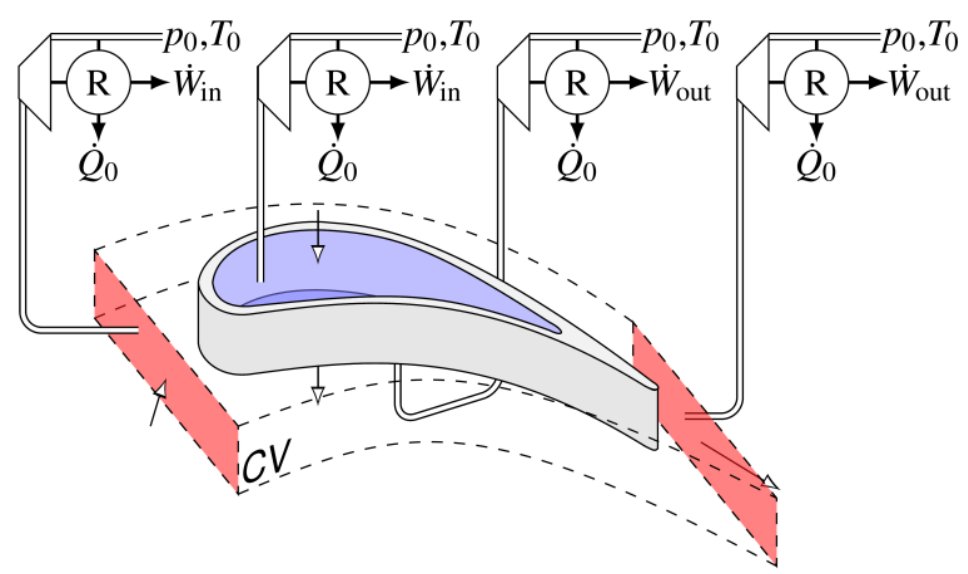

Figure 4.4 - Exergy method to defining loss coefficient.

$$
\begin{aligned}
& \omega^{\mathbf{e}}=\frac{\iiint \nabla \cdot\left(\mathbf{e}_{\mathbf{f}} \rho \boldsymbol{V}\right) d \mathrm{~V}}{\iint_{S_{\text {out }}} \rho \boldsymbol{V}\left(h_{t}-h\right) d S} \\
& \mathbf{e}_{\mathbf{f}}=\underbrace{\left[1-\frac{T_{0}}{T}\right]}_{\eta_{\text {th }}^{e}} h+\frac{V^{2}}{2}-T_{0}\left(s-s_{0}\right)
\end{aligned}
$$

If the euergy method is applied, the ideal work in Equation 4.3 is defined as the work that can be extracted by a reversible adiabatic turbine and is calculated at all of the inlets and outlets as shown in Figure 4.5. For this process, the thermal efficiency is set by the Joule cycle efficiency, $\eta_{t h}^{\mathbf{m}}$, resulting in the ideal flow work to become the flow euergy, $\mathbf{m}_{\mathbf{f}}$. For a steady flow, the euergy loss coefficient, $\omega^{\mathrm{m}}$, is defined by Equation 4.5.

$$
\begin{gathered}
\omega^{\mathbf{m}}=\frac{\iiint \nabla \cdot\left(\mathbf{m}_{\mathbf{f}} \rho \boldsymbol{V}\right) d \mathrm{~V}}{\iint_{S_{\text {out }}} \rho \boldsymbol{V}\left(h_{t}-h\right) d S} \\
\mathbf{m}_{\mathbf{f}}=\underbrace{\left[1-\left(\frac{p_{0}}{p}\right)^{\frac{\gamma-1}{\gamma}}\right]}_{\eta_{t h}^{\mathbf{m}}} h+\frac{V^{2}}{2}
\end{gathered}
$$

Throughout this thesis, a deliberate choice has been taken to demonstrate key concepts clearly. As a result, more detail into how the value placed on heat changes the ideal flow work of a general method has been included in Appendix A.1. In addition, a perfect gas has been used in all simulations. For the euergy method, the only effect of real gas properties is to change the value placed on heat, $\eta_{t h}^{\mathrm{m}}$, as shown in Appendix A.2. 


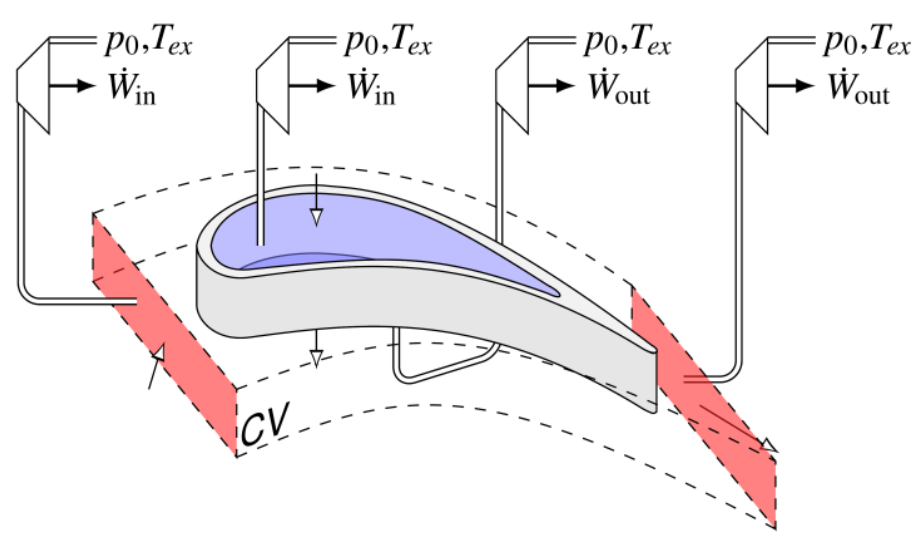

Figure 4.5 - Euergy method to defining loss coefficient.

The variation of exergy and euergy loss coefficient, $\omega^{\mathbf{e}}$ and $\omega^{\mathbf{m}}$ respectively, is shown in Figure 4.6. On the left, an uncooled turbine blade is examined operating at exit Mach numbers from 0.1 and 0.9 . This has been achieved by changing the exit pressure of the cascade to examine the applicability of the methods for compressible flow. On the right, a cooled turbine blade is examined operating at mainstream to coolant temperature ratios from 1 to 2 . This cascade operates at the design Mach number, but the total temperature of the coolant feed has been varied to examine the applicability of the methods to flows with heat transfer. For the uncooled turbine blade, both the exergy and euergy loss coefficients agree within $1.5 \%$. The similar behaviour indicates that both the exergy or euergy method would provide similar feedback to a turbine designer.

For the cooled turbine blade, as the mainstream-to-coolant temperature ratio varies from 1 to 2 , the exergy loss coefficient rises by $77.3 \%$ and the euergy loss coefficient drops by $3.7 \%$. This different behaviour would lead a turbine designer in a different direction.

The different behaviour exhibited by these methods stems from the difference in thermal efficiencies. Equation 4.6 illustrates that the difference between the two euergy and exergy methods is due to the difference in thermal efficiency times the transport of entropy. This result can be obtained from Equation A.11 in Appendix A.1.

$$
\left[\frac{\partial \mathbf{m} \rho}{\partial t}+\nabla \cdot\left(\mathbf{m}_{\mathbf{f}} \rho \boldsymbol{V}\right)\right]-\left[\frac{\partial \mathbf{e} \rho}{\partial t}+\nabla \cdot\left(\mathbf{e}_{\mathbf{f}} \rho \boldsymbol{V}\right)\right]=\left(\eta_{t h}^{\mathbf{m}}-\eta_{t h}^{\mathbf{e}}\right) \rho T \frac{D s}{D t}
$$



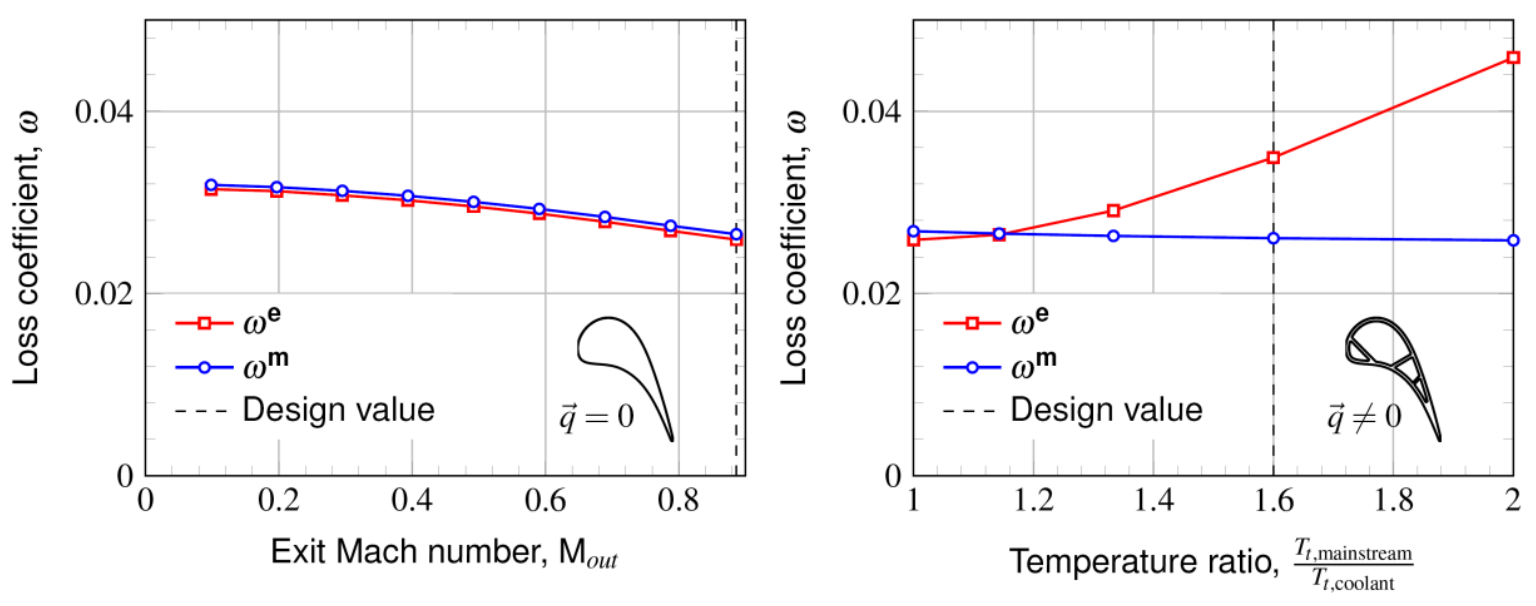

Figure 4.6 - The variation of exergy loss coefficient (red) and euergy loss coefficient (blue) with exit Mach number (left) and mainstream-to-coolant temperature ratio (right).

The difference between the exergy thermal efficiency and the euergy thermal efficiency is shown in Equation 4.7. When the changes in temperature and pressure are predominantly isentropic, the difference between the thermal efficiencies will be small. This means the exergy and euergy methods agree for highly-efficient adiabatic turbomachines as shown on the lefthand side of Figure 4.6. However, when there is an imposed temperature gradient, the changes in temperature and pressure are no longer isentropic so the difference between the thermal efficiencies becomes significant. This means the exergy and euergy method disagree for flows with heat transfer, such as cooled turbines, as shown on the right-hand side of Figure 4.6.

$$
\eta_{t h}^{\mathrm{m}}-\eta_{t h}^{\mathbf{e}}=\frac{T_{0}}{T}-\left(\frac{p_{0}}{p}\right)^{\frac{\gamma-1}{\gamma}}=\frac{T_{t}}{T}\left[\frac{T_{0}}{T_{t}}-\left(\frac{p_{0}}{p_{t}}\right)^{\frac{\gamma-1}{\gamma}}\right]
$$

In practice, measuring the loss coefficient is challenging in a high temperature and pressure environment. The loss coefficient is typically determined in a laboratory and then translated to engine. Industry methods for this translation account for the change in temperature, but not the increased rates of heat transfer. Implicit in this industrial practice is that the loss coefficient is relatively insensitive to heat transfer. This behaviour does not agree with the dramatic increase in loss coefficient exhibited by the exergy method. However, the euergy method chimes with the experience of turbine designers as it shows heat transfer has a much smaller effect on performance. 
For turbomachinery applications, the euergy method is the correct aerodynamic performance accounting method. This is because it both represents the thermodynamic process to which a turbine aspires (a reversible adiabatic expansion) and agrees with the behaviour experienced by industrial designers for over 60 years. The subsequent chapters will focus on applying the euergy method to examine the effect of heat transfer on turbine performance.

\subsection{Comparing industrially-focussed methods (euergy and power-balance)}

Accurately determining performance is of great importance to aviation and power generation communities. Until recently, the exergy method was the only method capable of linking subsystems to their effect on the overall system. Although the exergy method agrees with the physical reality for simple examples, it does not always agree with industrial experience (as demonstrated in the previous section for cooled turbine blades). This has led to a divide between the thermodynamic framework applied in academia and the practical experience in industry. Over the last decade, two new aerodynamic performance accounting methods have been proposed that seek to bridge the divide; the euergy method and the power-balance method.

The euergy method was developed by Miller (2013) for turbomachinery applications. As demonstrated in the previous section, this method is both aligned with the aims of a turbomachinery designer and agrees with industrial experience.

The power-balance method was developed by Drela (2009) for compressible viscous flow around an aircraft. This method is based on the difference between the mechanical power of the flow and the mechanical power of a freestream defined by a Trefftz plane. For aircraft design, the power-balance method has been well received as it appears to be aligned to the performance of an aircraft and allows tightly integrated systems to be clearly analysed. For turbomachinery flow, it is unlikely to be well suited as the concept of a uniform freestream is not applicable.

This section seeks to identify what the limitations of the power-balance method are. The power-balance loss coefficient is defined in Equation 4.8. 


$$
\omega^{\mathbf{p}}=\frac{\iiint \nabla \cdot\left(\boldsymbol{V}\left[\left(p-p_{\text {Trefftz }}\right)+\frac{1}{2} \rho\left(V^{2}-V_{\text {Trefftz }}^{2}\right)\right]\right) d V}{\iint_{S_{\text {out }}} \rho \boldsymbol{V}\left(h_{t}-h\right) d S}
$$

The reference Trefftz plane in this derivation plays a similar role to the reference environment in the general methods. To make the power-balance method general, a reference environment will be defined where $p_{0}=p_{\text {Trefftz }}$ and $V_{\text {Trefftz }}=0$.

With this reference environment, the difference in mechanical power can be written in the form of an ideal flow work, as shown in Equation 4.9. For the power-balance method, the thermal efficiency will be referred to as the power-balance thermal efficiency, $\eta_{t h}^{\mathbf{p}}$, resulting in the ideal flow work to become the flow power-balance, $\mathbf{p}_{\mathbf{f}}$. For a steady flow, the powerbalance loss coefficient, $\omega^{\mathbf{p}}$, is defined by Equation 4.9 and is mathematically equivalent to the loss coefficient defined previously by Equation 4.8.

$$
\begin{aligned}
& \omega^{\mathbf{p}}=\frac{\iiint \nabla \cdot\left(\mathbf{p}_{\mathbf{f}} \rho \boldsymbol{V}\right) d \mathrm{~V}}{\iint_{S_{\text {out }}} \rho \boldsymbol{V}\left(h_{t}-h\right) d S} \\
& \mathbf{p}_{\mathbf{f}}=\left[\frac{p-p_{0}}{\rho}+\frac{1}{2} V^{2}\right]=\underbrace{\left[\left(1-\frac{p_{0}}{p}\right) \frac{\gamma-1}{\gamma}\right]}_{\eta_{\text {th }}^{\mathbf{p}}} h+\frac{1}{2} V^{2}
\end{aligned}
$$

Comparing the euergy and power-balance equations, the difference is based on the difference in thermal efficiencies. This is shown in Equation 4.10. A detailed comparison between the two methods is provided in Appendix A.3.

$$
\left[\frac{\partial \mathbf{m} \rho}{\partial t}+\nabla \cdot\left(\mathbf{m}_{\mathbf{f}} \rho \boldsymbol{V}\right)\right]-\left[\frac{\partial \mathbf{p} \rho}{\partial t}+\nabla \cdot\left(\mathbf{p}_{\mathbf{f}} \rho \boldsymbol{V}\right)\right]=\left(\eta_{t h}^{\mathbf{m}}-\eta_{t h}^{\mathbf{p}}\right) \rho T \frac{D s}{D t}
$$

To further explore the difference between the two methods, let us consider their thermal efficiencies. The thermal efficiency of the euergy method considers an expansion between two thermodynamic states while the power-balance method considers the difference between two states. The effect of this can be seen by considering the pressure of the flow to be a small perturbation from the environment pressure (i.e. $p=p^{\prime}+p_{0}$ ). 
Equation 4.11 examines a small perturbation to the euergy thermal efficiency:

$$
\eta_{t h}^{\mathrm{m}}=1-\left(1+\frac{p^{\prime}}{p_{0}}\right)^{-\frac{\gamma-1}{\gamma}}=\frac{\gamma-1}{\gamma}\left[\frac{p^{\prime}}{p_{0}}-\frac{1}{2} \frac{2 \gamma-1}{\gamma}\left(\frac{p^{\prime}}{p_{0}}\right)^{2}+O\left(\left(\frac{p^{\prime}}{p_{0}}\right)^{3}\right)\right]
$$

Equation 4.12 examines a small perturbation to the power-balance thermal efficiency:

$$
\eta_{t h}^{\mathbf{p}}=\frac{\gamma-1}{\gamma}\left[1-\left(1+\frac{p^{\prime}}{p_{0}}\right)^{-1}\right]=\frac{\gamma-1}{\gamma}\left[\frac{p^{\prime}}{p_{0}}-\left(\frac{p^{\prime}}{p_{0}}\right)^{2}+O\left(\left(\frac{p^{\prime}}{p_{0}}\right)^{3}\right)\right]
$$

Comparing the difference between the two methods:

$$
\eta_{t h}^{\mathbf{m}}-\eta_{t h}^{\mathbf{p}}=\frac{\gamma-1}{\gamma} \frac{1}{2 \gamma}\left(\frac{p^{\prime}}{p_{0}}\right)^{2}+\cdots O\left(\left(\frac{p^{\prime}}{p_{0}}\right)^{3}\right)
$$

The difference between these two methods grows as the pressure deviates from the reference environment as shown in Equation 4.13. This is because the difference in mechanical power considered by the power-balance is a linearization of the euergy method for small expansions. As the pressure ratio of the system to environment grows, this linearization is no longer accurate. For an adiabatic flow with small temperature and pressure gradients, the error is approximately shown in Equation 4.14.

$$
\frac{\omega^{\mathbf{m}}-\omega^{\mathbf{p}}}{\omega^{\mathbf{m}}} \approx \frac{1}{2 \gamma} \frac{p-p_{0}}{p_{0}}
$$

The difference can be further examined by considering the loss mechanisms as shown in Equation 4.15. For highly efficient flows with no heat or work input, the first term on the right-hand side is small. This is particularly true when the pressure is close to the environmental such that the euergy thermal efficiency is also small. However, even under these conditions, the final term may not be. 


$$
\begin{aligned}
\nabla \cdot\left(\mathbf{m}_{\mathbf{f}} \rho \boldsymbol{V}\right)-\nabla \cdot\left(\mathbf{p}_{\mathbf{f}} \rho \boldsymbol{V}\right) & =\eta_{t h}^{\mathbf{m}}\left\{\left[\rho \frac{D h_{t}}{D t}-\frac{\partial p}{\partial t}\right]-\nabla \cdot\left(\mathbf{p}_{\mathbf{f}} \rho \boldsymbol{V}\right)\right\} \\
& -\left(1-\eta_{t h}^{\mathbf{m}}\right)\left[p-p_{0}\right](\nabla \cdot \boldsymbol{V})
\end{aligned}
$$

The final term in Equation 4.15 is a combination of how far the pressure deviates from the environment pressure and the divergence of velocity. The divergence of velocity is small for steady, incompressible flows. However, this becomes significant for compressible flow.

The divergence of velocity can be inspected using the continuity equation. Based upon scaling arguments, Greitzer, Tan, \& Graf (2004) illustrated that this term scales with Mach number squared for adiabatic flow. This is shown in Equation 4.16.

$$
\nabla \cdot \boldsymbol{V}=-\frac{1}{\rho} \frac{D \rho}{D t} \sim\left[\frac{\Delta \rho}{\rho}\right] /\left[\frac{\Delta V}{V}\right] \sim \mathrm{M}^{2}
$$

This loss mechanism in the power-balance method does not represent a reduction in efficiency of the practical device. For turbomachinery flows, both the pressure varies significantly, and the Mach numbers can be high. For external flows, the pressure varies less significantly even though the Mach numbers are high.

The power-balance method can be adapted to remove this loss mechanism. This has been achieved by examining the mass flow rate rather than the volumetric flow rate of the pressure difference. The difference between the power-balance method and this adapted powerbalance method are shown in Equation 4.17

$$
\nabla \cdot\left(\mathbf{p}_{\mathbf{f}} \rho \boldsymbol{V}\right)-\underbrace{\left[\frac{\nabla \cdot\left(\rho \boldsymbol{V}\left(p-p_{0}\right)\right)}{\rho}+\nabla \cdot\left(\rho \boldsymbol{V} \frac{V^{2}}{2}\right)\right]}_{\text {Adapted power-balance method }}=\left(p-p_{0}\right) \nabla \cdot \boldsymbol{V}
$$

Comparing the euergy method to this adapted power-balance method, Equation 4.18 shows that the unrepresentative loss mechanism has been successfully removed. However, there is now a density dividing the pressure difference term. 


$$
\begin{aligned}
\nabla \cdot\left(\mathbf{m}_{\mathbf{f}} \rho \boldsymbol{V}\right)- & {\left[\frac{\nabla \cdot\left(\rho \boldsymbol{V}\left(p-p_{0}\right)\right)}{\rho}+\nabla \cdot\left(\rho \boldsymbol{V} \frac{V^{2}}{2}\right)\right] } \\
& =\eta_{t h}^{\mathbf{m}}\left\{\left[\rho \frac{D h_{t}}{D t}-\frac{\partial p}{\partial t}\right]-\frac{\nabla \cdot\left(\rho \boldsymbol{V}\left(p-p_{0}\right)\right)}{\rho}+\nabla \cdot\left(\rho \boldsymbol{V} \frac{V^{2}}{2}\right)\right\}
\end{aligned}
$$

The density below the divergence causes difficulty when attempting to translate the volume integral to a surface integral. This expression indicates that the divergence and density must be known for every sub-element, not just the overall boundary of the system. This adapted power-balance method may have eliminated the loss mechanism, but it has also lost its generality.

The adapted power-balance method can be expressed as a loss coefficient, as shown in Equation 4.19. A constant reference density has been used, to allow the loss coefficient to be evaluated on the surface of the control volume.

$$
\omega^{\mathbf{p}^{\prime}}=\frac{\frac{1}{\rho_{\text {ref }}} \iiint \nabla \cdot\left(\rho \boldsymbol{V}\left(p-p_{0}\right)\right) d \mathrm{~V}+\iiint \nabla \cdot\left(\rho \boldsymbol{V} \frac{V^{2}}{2}\right) d \mathrm{~V}}{\iint_{S_{\text {out }}} \rho \boldsymbol{V}\left(h_{t}-h\right) d S}
$$

The variation of euergy loss coefficient, $\omega^{\mathbf{m}}$, power-balance loss coefficient, $\omega^{\mathbf{p}}$, and the bound of the adapted power-balance loss coefficient, $\omega^{\mathbf{p}}$, can be seen in Figure 4.7.

On the left, the power-balance loss coefficient deviates from the euergy loss coefficient as the Mach number increases. The peak Mach number is greater than the exit Mach number, so the effects of compressibility are seen even for relatively low exit Mach numbers. This deviation can either be viewed as the breakdown of the linearization or as a result of the divergence of velocity loss mechanism. The power-balance method is not suited to turbomachinery flows except when adiabatic and the Mach number are low.

On the right, the bound of possible adapted power-balance loss coefficients is shown. This has been achieved by taking the smallest and greatest density experienced within the turbine cascade as the reference density in Equation 4.19. For low Mach numbers, this bound 

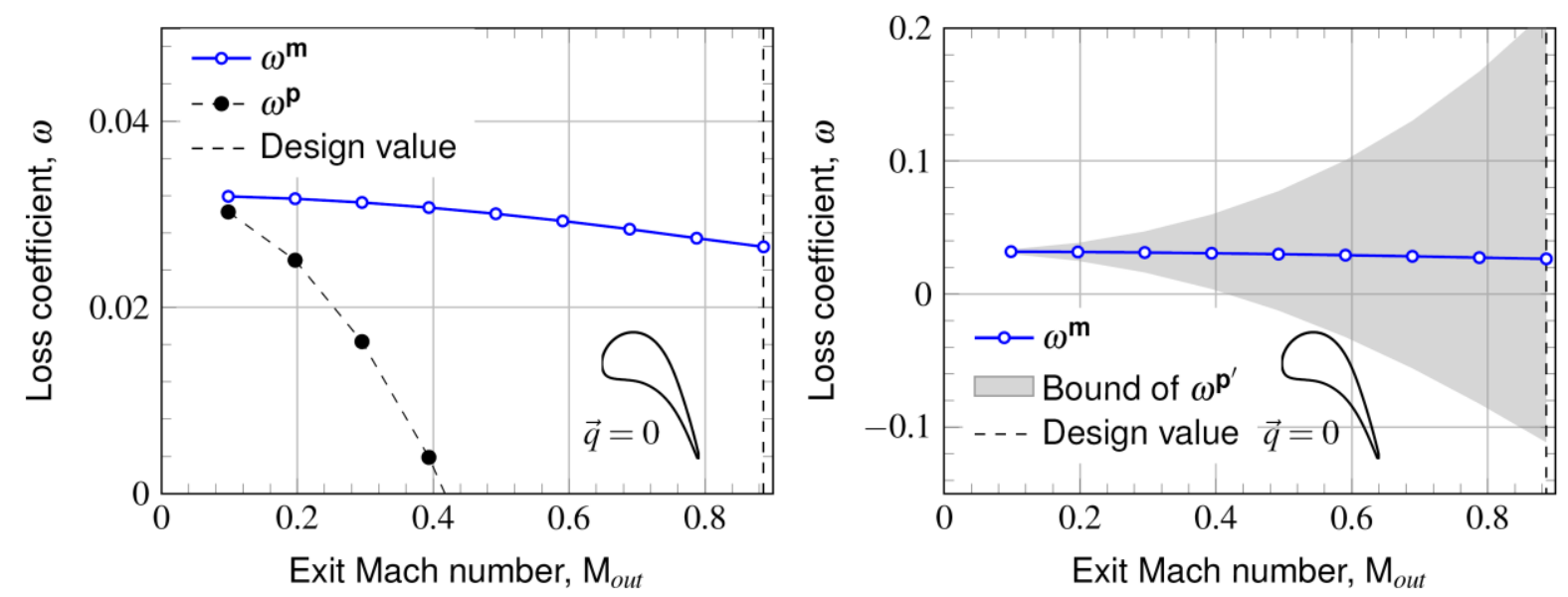

Figure 4.7 - The variation of euergy loss coefficient (blue) with exit Mach number compared to the powerbalance loss coefficient (left) and the bound of the adapted power-balance loss coefficient (right).

could be a useful tool. However, it mainly demonstrates the extent to which the power-balance method misinforms a designer at high Mach numbers.

It has been shown that the power-balance method is a linearization of the euergy method that is only accurate for small deviations in pressure from the reference environment. The effect is particularly pronounced at high Mach numbers and can be attributed to either the divergence of velocity or density variation.

For turbomachinery applications, the power-balance method has limited application as the pressure can change by $60 \%$ across a stage. For flows around an aircraft, the pressure eventually returns to the freestream condition. However, it is unclear whether the linearization is still accurate at high Mach numbers or when a limited control volume is examined (as in CFD). In the subsequent chapters, the euergy method will be used to examine cooled turbines.

\subsection{Extending adiabatic loss coefficients to cooled turbines}

Loss coefficients are widely used to measure the performance of uncooled turbines. However, it is not clear how they relate to cooled turbine performance or how to attribute loss to regions of the flow. In this section, three commonly used loss coefficients will be examined; the enthalpy loss coefficient, entropy loss coefficient and stagnation pressure loss coefficient. 


\subsubsection{Enthalpy loss coefficient}

The enthalpy loss coefficient, or energy loss coefficient, is a useful metric for design purposes. It considers the difference between the actual exhaust state and the exhaust state of an ideal turbine. As the enthalpy loss coefficient is typically applied to steady, stationary, adiabatic blade rows, this metric could be viewed as the difference in ideal work from the inlet compared to outlet as shown in Equation 4.20.

$$
\omega^{h}=\frac{\sum_{\text {out }} \overline{h_{s, p_{0}}}-\sum_{\text {in }} \overline{h_{s, p_{0}}} \mathrm{~m}}{\sum_{\text {out }} \bar{h}_{t}-h^{\mathrm{m}}} \approx \frac{\sum_{\mathrm{in}} \overline{h_{t}-h_{s, p_{0}}} \mathrm{~m}-\sum_{\text {out }} \overline{h_{t}-h_{s, p_{0}}} \mathrm{~m}}{\sum_{\text {out }} \bar{h}_{t}-h^{\mathrm{m}}}
$$

The enthalpy loss coefficient considers the ideal work to be the work that could be extracted by a reversible adiabatic turbine exhausting to the pressure of the cascade outlet. This view of ideal work is aligned with the euergy method. Equation 4.21 highlights the enthalpy loss coefficient is an instance of this general method.

$$
\omega^{h} \rightarrow \omega^{\mathbf{m}}=\frac{\iiint \nabla \cdot\left(\mathbf{m}_{\mathbf{f}} \rho \boldsymbol{V}\right) d \mathrm{~V}}{\iint_{S_{\text {out }}} \rho \boldsymbol{V}\left(h_{t}-h\right) d S}
$$

The euergy method can be used to extend the enthalpy loss coefficient to examine more complex flows.

\subsubsection{Entropy loss coefficient}

The entropy loss coefficient is based upon the change in entropy due to irreversible flow processes across the cascade. It considers all entropy creation as detrimental to turbine performance. As the entropy loss coefficient is typically applied to steady, stationary, adiabatic blade rows, this metric could also be viewed as the difference in ideal work from the inlet compared to outlet as shown in Equation 4.22.

$$
\omega^{s}=\frac{T_{0} \Delta s}{\sum_{\text {out }} \bar{h}_{t}-h^{\mathrm{m}}} \approx \frac{\sum_{\mathrm{in}} \overline{h_{t}-h_{T_{0}, p_{0}}+T_{0} s} \mathrm{~m}-\sum_{\text {out }} \overline{h_{t}-h_{T_{0}, p_{0}}+T_{0} s} \mathrm{~m}}{\sum_{\text {out }} \bar{h}_{t}-h^{\mathrm{m}}}
$$


The entropy loss coefficient considers the ideal work to the work that could be extracted by a universal reversible machine exhausting to the pressure and temperature of the cascade outlet. This view of ideal work is aligned with the exergy method. Equation 4.23 highlights the entropy loss coefficient is an instance of this general method.

$$
\omega^{S} \rightarrow \omega^{\mathbf{e}}=\frac{\iiint \nabla \cdot\left(\mathbf{e}_{\mathbf{f}} \rho \boldsymbol{V}\right) d \mathrm{~V}}{\iint_{S_{\text {out }}} \rho \boldsymbol{V}\left(h_{t}-h\right) d S}
$$

The exergy method can be used to extend the entropy loss coefficient. This concept has been used by many researchers as exergy is an established thermodynamic framework.

\subsubsection{Stagnation pressure loss coefficient}

The stagnation pressure loss coefficient is possibly the most common loss coefficient as it is easy to calculate. It considers the difference in stagnation pressure at the inlet and exit as shown in Equation 4.24. For incompressible flow, the stagnation pressure represents the mechanical energy of the flow. As a result, this could be viewed as an incompressible version of the enthalpy loss coefficient.

$$
\omega^{p}=\frac{\sum_{\text {in }}{\overline{p_{t}}}^{\mathrm{m}}-\sum_{\text {out }} \bar{p}_{t}^{\mathrm{m}}}{\sum_{\text {out }}{\overline{p_{t}-p^{\mathrm{m}}}}^{\mathrm{m}}}=\frac{\sum_{\text {in }}{\overline{p_{t}-p_{0}}}^{\mathrm{m}}-\sum_{\text {out }} \overline{p_{t}-p_{0}}}{\sum_{\text {out }}^{\mathrm{m}}}
$$

Converting into an integral form, the stagnation pressure loss coefficient is expressed by Equation 4.25 .

$$
\omega^{p}=\frac{\iiint \nabla \cdot\left(\rho \boldsymbol{V}\left(p_{t}-p_{0}\right)\right) d \boldsymbol{V}}{\iint_{S_{\text {out }}} \rho \boldsymbol{V}\left(p_{t}-p\right) d S}
$$

This form is not readily extended using a generalizable method. Comparing with the euergy method for a steady flow with $\eta_{t h}^{m} \rightarrow 0$, Equation 4.26 shows that the numerator of the stagnation pressure loss coefficient must be divided by density. As the density could vary, this restricts transforming the volume integral to the control volume surface. 
The final term in Equation 4.26 indicates the difference between the euergy and stagnation pressure loss coefficients for constant density flow. For low Mach numbers, this term is small. Also, for many flows the changes in stagnation pressure are predominantly caused by velocity gradients. This means that this term will be small for highly-efficient components.

$$
\nabla \cdot\left(\mathbf{m}_{\mathbf{f}} \rho \boldsymbol{V}\right)-\frac{\nabla \cdot\left(\rho \boldsymbol{V}\left(p_{t}-p_{0}\right)\right)}{\rho}=-\left(1-\frac{\rho}{\rho_{t}}\right) V \cdot \nabla p_{t}
$$

Translating this understanding back into the stagnation pressure loss coefficient, Equation 4.27 highlights the need for a reference density. In the traditional loss coefficient, this is implicitly set by the outlet. This updated stagnation pressure loss coefficient instead presents a bound of possible loss coefficients.

$$
\omega^{p^{\prime}}=\frac{\frac{1}{\rho_{\text {ref }}} \iiint \nabla \cdot\left(\rho \boldsymbol{V}\left(p_{t}-p_{0}\right)\right) d V}{\iint_{S_{\text {out }}} \rho \boldsymbol{V}\left(h_{t}-h\right) d S} \approx \frac{\bar{\rho}^{\text {out }}}{\rho_{\text {ref }}} \frac{{\overline{p_{t}}}^{\text {m,in }}-\bar{p}_{t}^{\text {m,out }}}{{\overline{p_{t}}}^{\text {m,out }}-\bar{p}^{\text {A,out }}}=\frac{\bar{\rho}^{\text {out }}}{\rho_{\text {ref }}} \omega_{p}
$$

This is like the power-balance method. There, the density was a result of absorbing a loss mechanism. This can also be done in reverse. The density implicit in the stagnation pressure loss coefficient can be viewed as a combination of a quasi-general method and a loss mechanism.

$$
\underbrace{\nabla \cdot\left(\rho \boldsymbol{V}\left(\frac{p_{t}-p_{0}}{\rho}\right)\right)}_{\text {generalised method }}-\frac{\nabla \cdot\left(\rho \boldsymbol{V}\left(p_{t}-p_{0}\right)\right)}{\rho}=\left(p_{t}-p_{0}\right) \nabla \cdot \boldsymbol{V}
$$

The first term in Equation 4.28 indicates the ideal flow work. This is further examined in Equation 4.29. For low Mach number flows, this quasi-general method is the power-balance method. 


$$
\begin{aligned}
& \mathbf{y}_{\mathbf{f}}=\frac{p_{t}-p_{0}}{\rho}=\underbrace{\left[1-\frac{p_{0}}{p}+\frac{\gamma}{8} M^{4}+O\left(M^{6}\right)\right] \frac{\gamma-1}{\gamma}}_{\eta_{t h}^{\mathrm{y}}} h+\frac{V^{2}}{2} \\
& \eta_{t h}^{\mathbf{y}}=\eta_{t h}^{\mathrm{p}}+\left[\frac{\gamma}{8} M^{4}+O\left(M^{6}\right)\right] \frac{\gamma-1}{\gamma}
\end{aligned}
$$

The final term in Equation 4.28 demonstrates the new loss mechanism. Unless the Mach number is very small, this term is large as both the stagnation pressure is not close to the static pressure and the divergence of velocity is not small.

$$
\nabla \cdot\left(\mathbf{m}_{\mathbf{f}} \rho \boldsymbol{V}\right)-\nabla \cdot\left(\mathbf{y}_{\mathbf{f}} \rho \boldsymbol{V}\right)=\left(p_{t}-p_{0}\right)(\nabla \cdot \boldsymbol{V})-\left(1-\frac{\rho}{\rho_{t}}\right) V \cdot \nabla p_{t}
$$

Equation 4.30 shows the difference between the euergy and quasi-general method. The variation of euergy loss coefficient, $\omega^{\mathrm{m}}$, stagnation pressure loss coefficient, $\omega^{p}$, and the bound of updated stagnation pressure loss coefficient, $\omega^{p^{\prime}}$, can be seen in Figure 4.8.

On the left, the stagnation pressure loss coefficient deviates from the euergy loss coefficient as the Mach number increases. The trend of how the loss coefficient behaves is also different for the two methods. This deviation is predominantly due to the outlet density not representing the reference density.
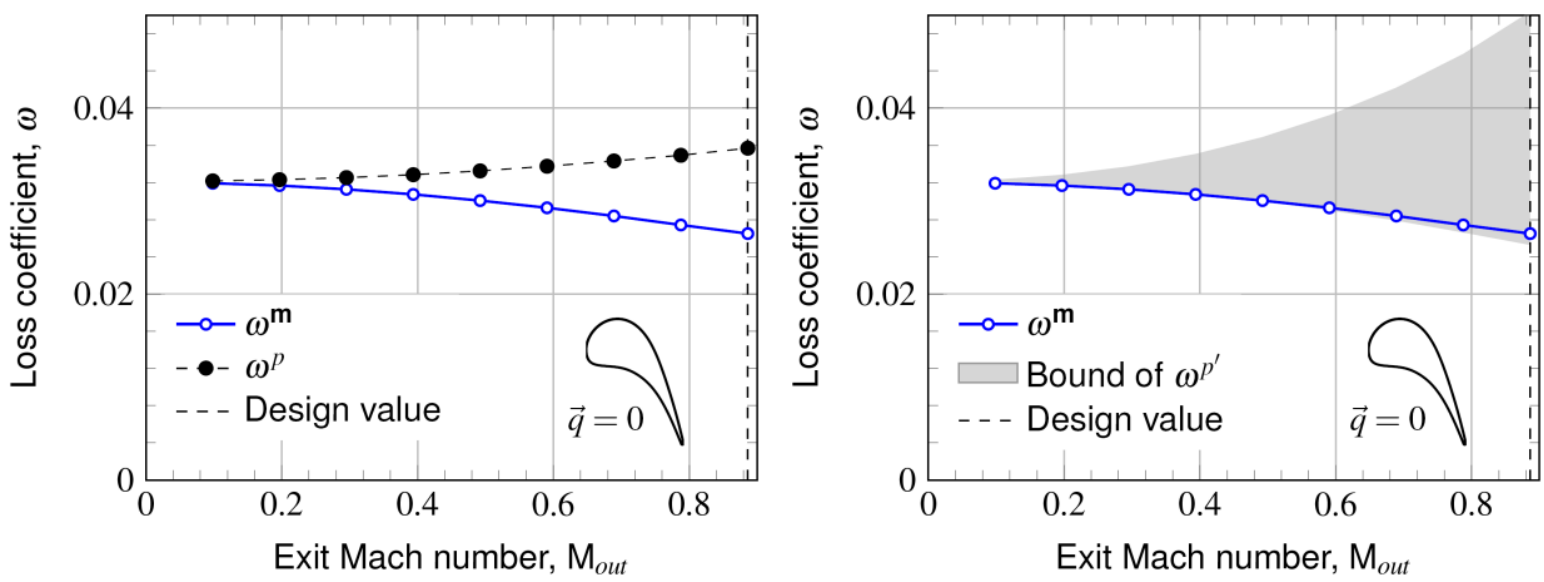

Figure 4.8 - The variation of euergy loss coefficient (blue) with exit Mach number compared to the stagnation pressure loss coefficient (left) and the bound of the updated loss coefficient (right). 
On the right, the bound of possible updated stagnation pressure loss coefficients are shown. This has been achieved by taking the smallest and greatest density experienced within the turbine cascade as the reference density in Equation 4.27. The greatest density was found at the inlet. If this is taken as the reference density, the euergy loss coefficient and stagnation pressure loss coefficient agree well. This is useful to gauge the accuracy of the stagnation pressure loss coefficient.

It is worth noting here that stagnation pressure can be used to accurately determine the euergy loss coefficient. The same measurement information is required. Alternatively, the stagnation pressures could be used to calculate the exergy loss coefficient using a linearization presented by Denton (1993).

The power-balance method could be used to extend an adapted stagnation pressure loss coefficient. However, this results in a considerable loss of accuracy for turbomachinery flows. Instead, the applicability of the stagnation pressure loss coefficient can be assessed using the ratio of the maximum and minimum densities in the flow. This indicates that the stagnation pressure loss coefficient becomes less applicable when cooling is involved as the ratio of densities becomes greater. A more accurate solution would be to use the stagnation pressure information to calculate the euergy loss coefficient directly.

\subsection{Summary}

This chapter compared the exergy, euergy and power-balance methods, identified their key differences and demonstrated their ability to extend traditional loss coefficients to cooled turbines. The comparison examined what each method regarded the ideal flow work to be. The key difference between these methods is the relative increase in ideal work due to a heat addition; i.e. the value of heat. The euergy method is capable of capturing the performance of a cooled turbine. This method both captures the aspirations of a cooled turbine and agrees with industrial experience.

When the exergy and euergy methods were compared, the only difference was the value they placed on heat. The exergy method values heat based upon the thermal efficiency of the 
Carnot cycle. This assumes that a designer is aspiring to a device comprised of both a reversible adiabatic turbine and a reversible heat engine. The euergy method values heat based upon the thermal efficiency of the Joule (Brayton) cycle. This assumes that a designer has access to a reversible adiabatic turbine. The euergy method reflects the aims of a turbine designer and was shown to agree with industrial experience. The exergy method does not reflect turbine performance when there is heat transfer and could be misleading to a designer.

When the euergy and power-balance methods were compared, the difference again stemmed from the value of heat and appeared when the pressure of the flow is not close the pressure of the reference environment. The euergy method defines the value of heat based upon a reversible adiabatic expansion. The power-balance method defines the value of heat based upon a linearization of this process. For very small changes in pressure, this linearization is accurate. However, this is not typically the case in turbomachinery. The power-balance method does not reflect turbine performance when there is heat transfer or the flow is compressible.

Finally, the ability to extend three traditional loss coefficients (the enthalpy, entropy and stagnation pressure loss coefficients) to cooled turbines was considered. The key difference between loss coefficients was what they regard to be the ideal work that could be extracted from a flow. These loss coefficients are aligned to the more general methods. The enthalpy loss coefficient could be extended using the euergy method. The entropy loss coefficient could be extended using the exergy method. Finally, there are strong parallels between the stagnation pressure loss coefficient and the power-balance method. These general methods allow the traditional loss coefficients to be broken down into regions of the flow. The applicability of the traditional loss coefficients reflects the more general method to which they belong. Only the extended enthalpy loss coefficient, based upon the euergy method, is able to determine the effect of heat transfer on turbine performance.

The following chapters will focus on applying the euergy method to examine the effect of heat transfer on turbine performance. 



\section{Chapter 5 Loss mechanisms in cooled turbines}

The flow features responsible for changing the performance of cooled turbines are unclear. Historically, these loss mechanisms have been linked to performance either by decomposing the stagnation pressure loss coefficient or by using the exergy method. However, the previous section demonstrated that both methods are not accurate for cooled turbines. This chapter will apply the euergy method to examine how heat transfer affects the loss mechanisms present in a cooled turbine cascade. For the first time, the nature of these loss mechanisms can be understood. This chapter is divided into three sections:

First, the effect of heat transfer between streams is examined. This occurs both between stream-tubes within the flow and between mainstream and coolant streams. Currently, it is unclear whether heat transfer between streams of different temperature, thermal dissipation, affects turbine performance. In this section, it is shown that it is the effect of heat transfer between streams of different pressure, thermal creation, is important.

Second, the reheat effect due to viscous dissipation is examined. This occurs as kinetic energy is dissipated into internal energy. This could be due to either mean or fluctuating flow structures. The dissipation coefficient in this thesis is defined as the sum of the effect of mean dissipation and the effect of the production of turbulent kinetic energy. Proponents of neglecting thermal dissipation usually advocate only using the viscous loss mechanisms of the exergy method. The accuracy of this approach for cooled turbines is unknown.

Finally, the effect of heat transfer on viscous dissipation is examined. This occurs as heat transfer results in temperature gradients which cause the thermofluid properties to change. This is a complex interaction that will be examined further in Chapter 7. 


\subsection{Heat transfer between streams}

The loss coefficient of a blade row can be reduced by heat transfer. Theoretically, the loss coefficient could even be negative. This may seem strange, but the increase in work that could be extracted from the flow comes from recovering energy from the hot turbine exhaust. Locally within the flow, the effect of heat transfer on performance can be thought of as a form of recuperation; recovering energy from the turbine exhaust that would not have been used.

The physical mechanism behind this effect is demonstrated in the example in Figure 5.1. Consider a small heat flux, $\delta \dot{Q}$, passed between two streams of a perfect gas. If the hot stream has a greater pressure than the cold stream, heat would have been transferred from a flow with a lower Joule cycle efficiency to a flow with a higher Joule cycle efficiency. If the heat is passed into the higher Joule cycle efficiency flow, an ideal turbine could extract more work from the same amount of heat. This heat transfer would result in the work which can be extracted from the flow rising by:

$$
\left[\left(\frac{p_{0}}{p_{\text {low }}}\right)^{\frac{\gamma-1}{\gamma}}-\left(\frac{p_{0}}{p_{\text {high }}}\right)^{\frac{\gamma-1}{\gamma}}\right] \delta \dot{Q}
$$

For a fluid element of perfect gas, Equation 5.1 can be expressed in differential form:

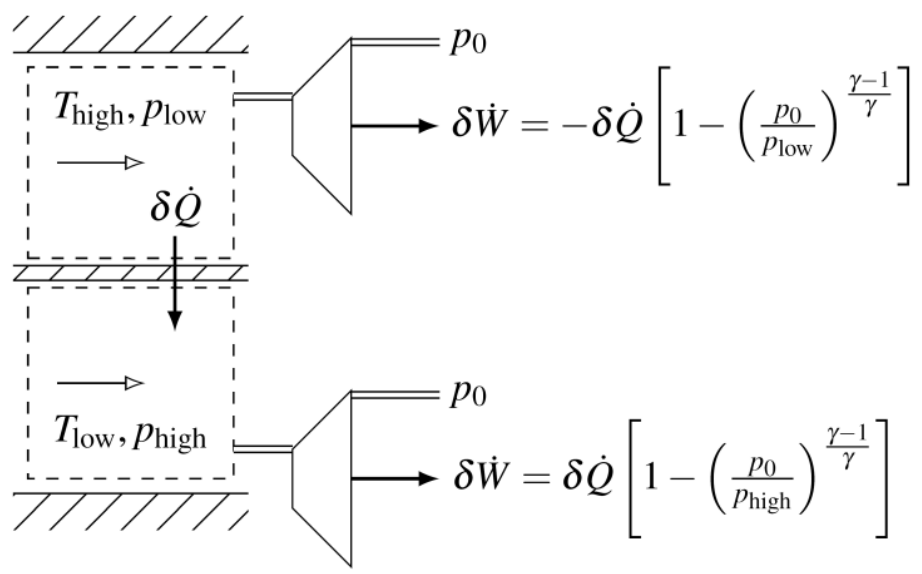

Figure 5.1 - The effect of transferring a small heat flux between two streams on the ideal work of a turbine. 


$$
\Phi_{t h}^{\mathrm{m}}=\left(\frac{p_{0}}{p}\right)^{\frac{\gamma-1}{\gamma}} \underbrace{\left[\boldsymbol{q} \cdot \frac{\gamma-1}{\gamma} \frac{\nabla p}{p}\right]}_{\Delta_{\text {therm }}}
$$

where $\boldsymbol{q}$ is the heat flux vector within the flow. The form presented in Equation 5.2 can now be integrated across the blade row control volume. This thermal loss mechanism for the euergy method was first presented by Miller as thermal creation, $\Delta_{t h e r m}$. It can be either positive or negative as heat can be transferred up or down pressure gradients. When heat is transferred up pressure gradients, the thermal loss mechanism for euergy, $\Phi_{t h}^{m}$, is positive and acts to increase turbine performance. This occurs when the temperature gradient and pressure gradient act in opposite directions.

Figure 5.2 shows how both thermal creation and viscous dissipation contribute to the euergy loss coefficient of a blade row when integrated across the control volume. At a mainstream-to-coolant temperature ratio of 1 , thermal creation is responsible for a $2.1 \%$ increase in loss. At a mainstream-to-coolant temperature ratio of 2 , thermal creation is responsible for a $7.0 \%$ reduction in loss. Therefore, the effect of heat transfer between streams acts to reduce the loss coefficient by $9.1 \%$ between a temperature ratio of 1 and 2 .

The thermal creation that occurs within the flow can be seen in the contours plotted in Figure 5.3, for a mainstream-to-coolant temperature ratio of 2 . The effect of thermal creation

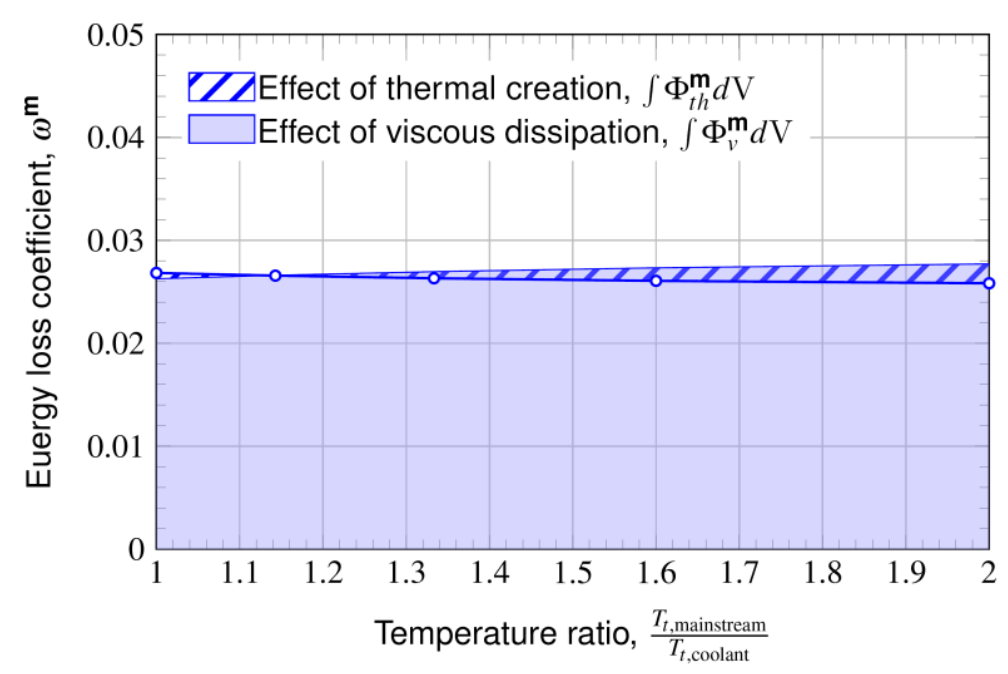

Figure 5.2 - The variation of the euergy loss coefficient with mainstream-to-coolant temperature ratio. 
has been normalised by the total blade loss divided by the suction surface boundary layer volume. The relative magnitude can be seen when compared to the effect of viscous dissipation in Figure 5.7. However, the effect of thermal creation within the flow is small relative to the effect due to heat transfer across the blade wall, which represents $98 \%$ of the total thermal creation. The reason for this is that a much larger difference in static pressure can be maintained across the wall than across the flow. The pressure difference is particularly great along the suction surface of the blade where the high Mach number results in reducing the static pressure in this region. This results in the effect of thermal recuperation being largest across the blade wall along the suction surface.

A similar analysis can be performed to examine the effect of heat transfer between streams on the exergy method. When small heat flux, $\delta \dot{Q}$, is passed between two streams, the second law of thermodynamics states that it must be passed from a higher to lower temperature. Therefore, the heat is transferred from a hot stream, with a higher Carnot cycle efficiency, to a cold stream, with a lower Carnot cycle efficiency. The resulting change in work from a small heat flux, $\delta \dot{Q}$, being passed between these streams is:

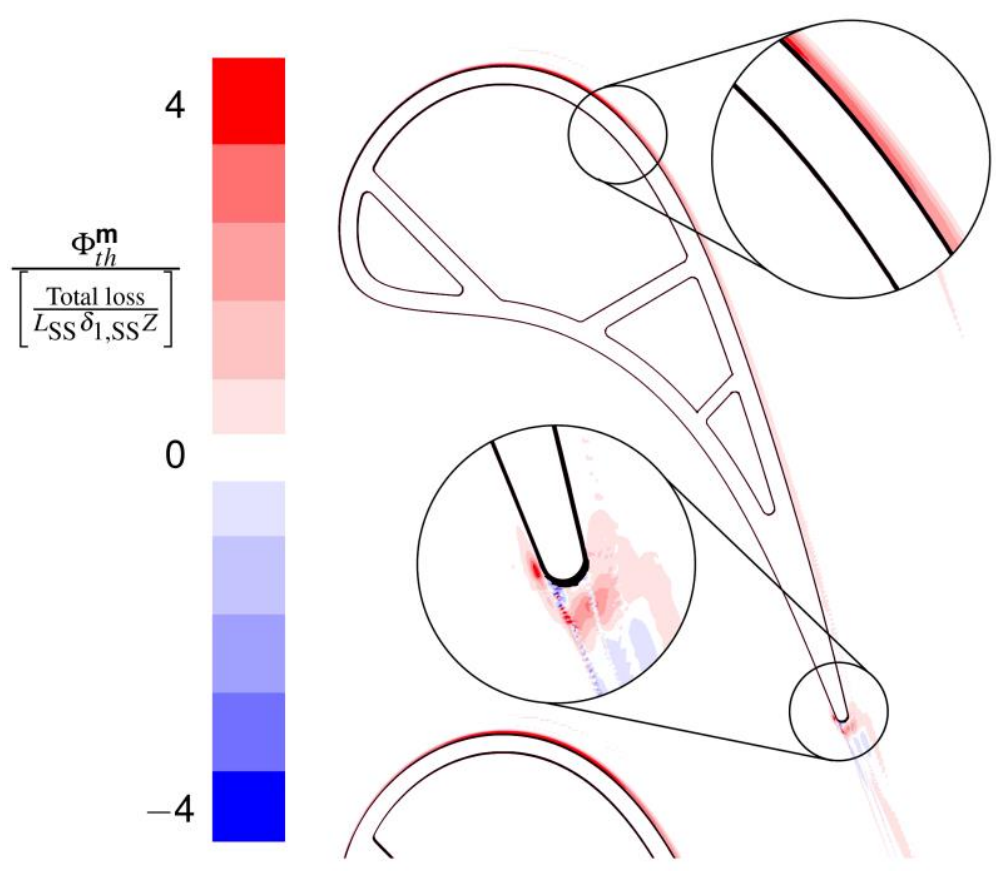

Figure 5.3 - Contours showing the thermal creation contribution to turbine loss (temperature ratio of 2). 


$$
\left[\frac{T_{0}}{T_{\text {high }}}-\frac{T_{0}}{T_{\text {low }}}\right] \delta \dot{Q}
$$

As heat can only be transferred from a higher to lower temperature, the term shown in Equation 5.3 always acts to increase the exergy loss coefficient. For a fluid element, Equation 5.3 can be written in differential form as:

$$
\Phi_{\text {th }}^{\mathbf{e}}=\frac{T_{0}}{T} \underbrace{\left[\boldsymbol{q} \cdot \frac{\nabla T}{T}\right]}_{-\phi_{\text {therm }}}
$$

The form in Equation 5.4 can now be integrated across the blade row control volume. This thermal loss mechanism for the exergy method is known as thermal dissipation, $\phi_{\text {therm }}$. It can only act to increase the exergy loss coefficient as heat is restricted to only be transferred from a higher to lower temperature. Therefore, the thermal loss mechanism for exergy, $\Phi_{t h}^{\mathbf{e}}$, can only be negative.

Figure 5.4 shows how both thermal dissipation and viscous dissipation contribute to the exergy loss coefficient of a blade row when integrated across the control volume. At a mainstream-to-coolant temperature ratio of 2 , thermal dissipation represents $33.3 \%$ of the total loss coefficient.

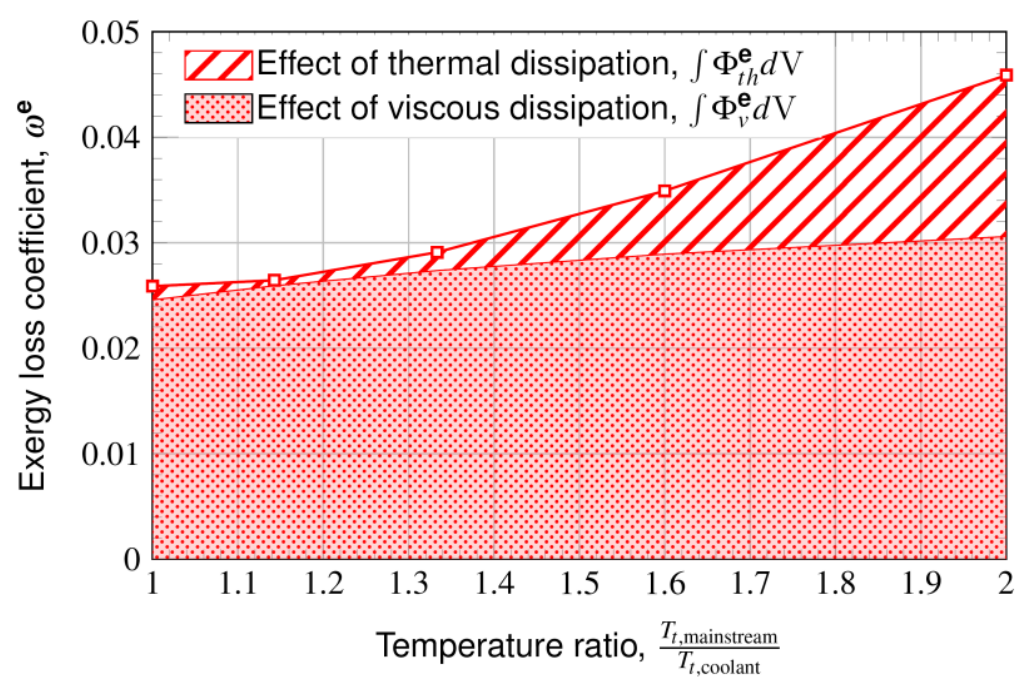

Figure 5.4 - The variation of the exergy loss coefficient with mainstream-to-coolant temperature ratio. 
The effect of heat transfer between streams can be more generally thought of as heat transfer across gradients of thermal efficiency, as shown in Equation 5.5 and Appendix A.1. This is a thermal recuperation effect, $\phi_{t h}$, as energy that would be contained in the exhaust is made available to the ideal device. For the euergy method, this occurs when heat is transferred up gradients of Joule cycle efficiency and is called thermal creation, $\phi_{t h}^{m}=\Delta_{t h e r m}$. For the exergy method, heat can only be transferred down gradients of Carnot cycle efficiency and this is called thermal dissipation, $\phi_{t h}^{\mathbf{e}}=-\phi_{t h e r m}$. The effect of recuperation has only previously been considered on a cycle design level. Using the euergy method, this effect can now be considered at a component design level allowing more efficient turbomachines.

$$
\phi_{t h}=\boldsymbol{q} \cdot \frac{\nabla \eta_{t h}}{1-\eta_{t h}}
$$

This section demonstrated that heat transfer in a cooled turbine acts to improve performance. The mechanism that causes this rise is predominantly due to heat transfer for the lower-pressure (high Mach number) mainstream to the higher-pressure coolant passage. This heat transfer across pressure gradients increases the work available to a turbine by reducing the waste exhaust energy. This recuperation effect is not currently included in component design and could be used to improve turbine efficiency.

\subsection{Viscous reheat}

The process of converting kinetic energy into internal energy is called viscous dissipation. This process is illustrated in Figure 5.5 and is often decomposed into laminar (molecular) and turbulent contributions. As the flow passes through a throttle, viscous mixing acts to reduce the kinetic energy of the flow and increase the internal energy by an equal magnitude. As an ideal device could extract all the kinetic energy, the reduction in ideal work is equal to the magnitude of the reduction of kinetic energy. As an ideal device could only extract a fraction of the internal energy, the increase in ideal work is a fraction of magnitude of the increase in internal energy. This increase in internal energy is equivalent to a heat addition and is known as the viscous reheat effect. The value placed on this heat addition sets the fraction of ideal work recovered. 
For the euergy method, the value of heat is set by the Joule cycle efficiency at the location where the viscous dissipation occurs. The change in the rate of work that can be extracted from a flow due to a small rate of dissipation of kinetic energy, $\delta \phi_{v}$, is given by:

$$
-\delta \phi_{v}+\left[1-\left(\frac{p_{0}}{p}\right)^{\frac{\gamma-1}{\gamma}}\right] \delta \phi_{v}=-\left(\frac{p_{0}}{p}\right)^{\frac{\gamma-1}{\gamma}} \delta \phi_{v}
$$

On the left-hand side of Equation 5.6, the second term shows the effect of the viscous reheat. The fraction of the ideal work recovered by this viscous reheat is set by the local Joule cycle efficiency.

In a cooled turbine, the most important effect is how the value of heat varies through the boundary layer. The value of heat is set by the euergy method using the Joule cycle efficiency and the exergy method using the Carnot cycle efficiency. Their variation through a cooled boundary layer is shown in Figure 5.6. As the euergy method uses the local static pressure, the Joule cycle efficiency does not vary significantly across the boundary layer. This means that, when the wall is cooled, viscous dissipation will have the same effect on the euergy loss coefficient. As the exergy method uses the local static temperature, the Carnot cycle efficiency changes across the boundary layer. This means, when the wall is cooled, the same amount of viscous dissipation will have a greater impact on the exergy loss coefficient.

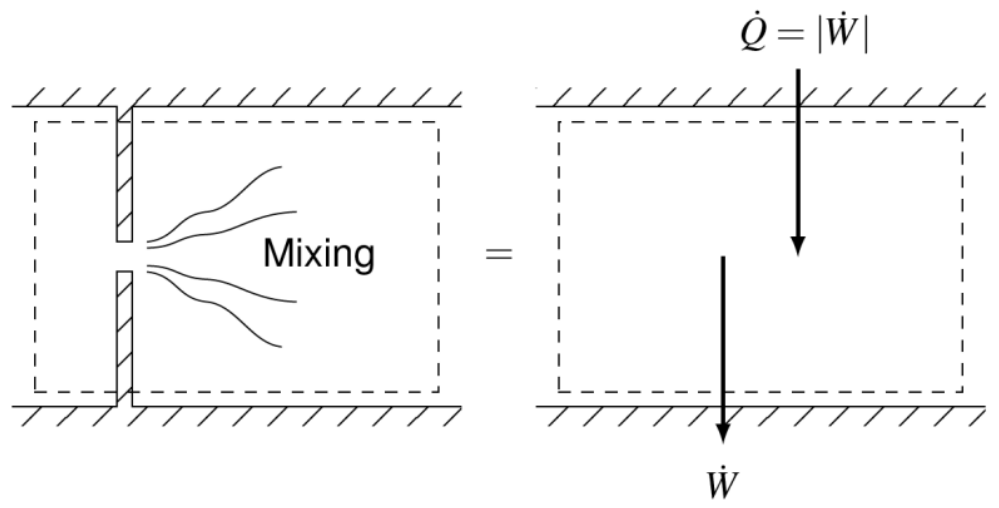

Figure 5.5 - Schematic showing viscous dissipation, such as that downstream of a throttle, is equivalent to work being removed from a flow and replaced by a heat addition of equal magnitude. 


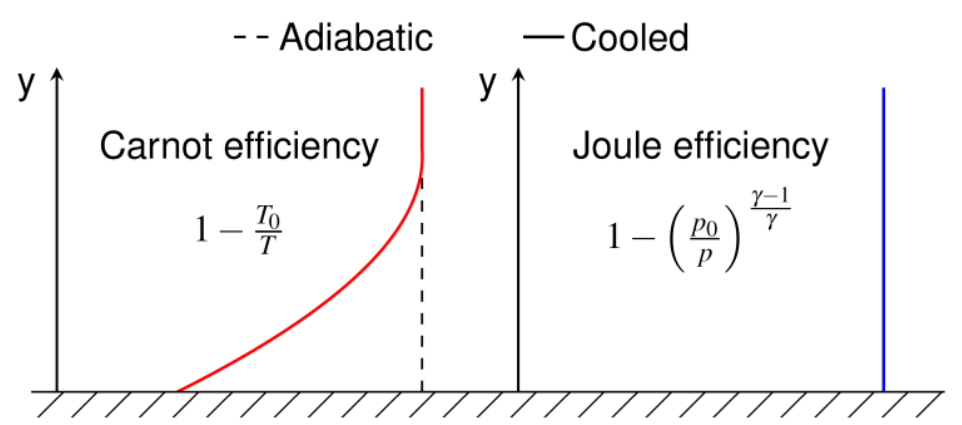

Figure 5.6 - The profile of Carnot cycle efficiency and Joule cycle efficiency for a cooled boundary layer.

Where viscous dissipation acts to reduce turbine performance can be seen in Figure 5.7. The effect of viscous dissipation has been normalised by the total blade loss divided by the suction surface boundary layer volume and has a much greater magnitude than the effect of thermal creation shown in Figure 5.3. Most of this effect occurs along the suction side boundary layer and in the downstream wake.

Figure 5.8 shows how viscous dissipation contributes to the euergy and exergy loss coefficient of a blade row when integrated across the control volume. The viscous component of the euergy loss coefficient only rises by $5.5 \%$ as the mainstream-to-coolant temperature ratio

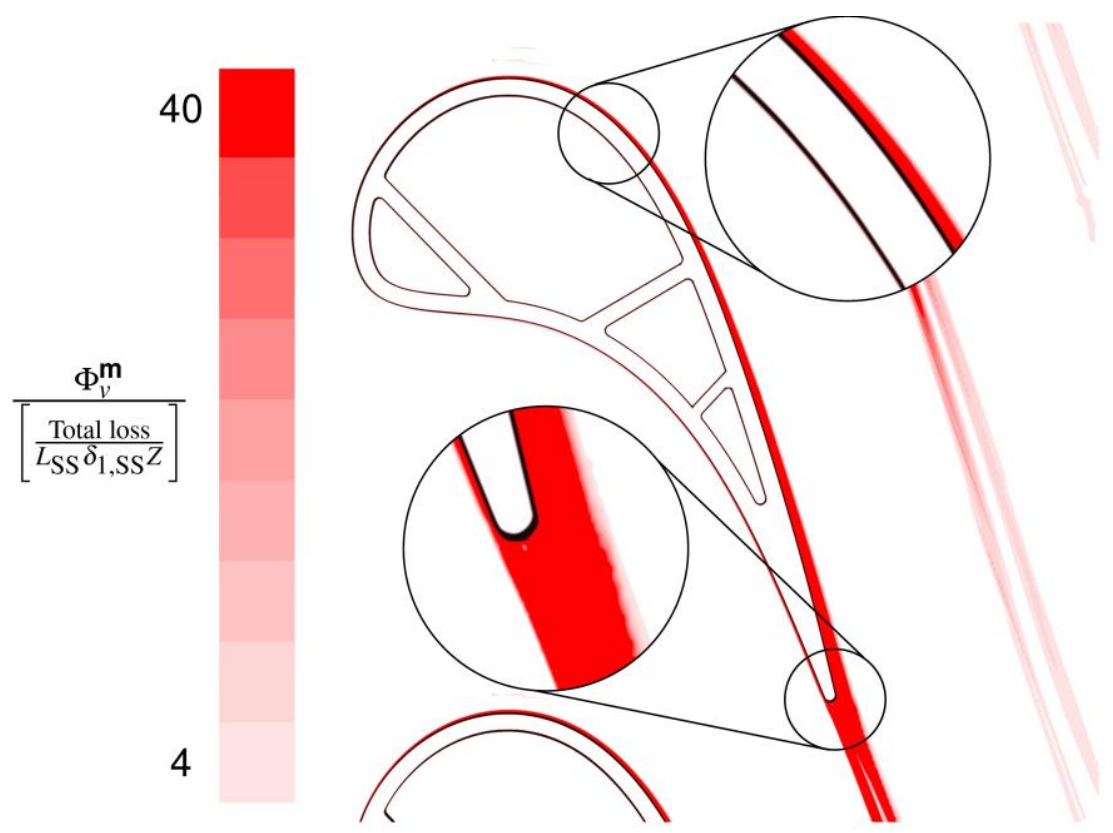

Figure 5.7 - Contours showing the viscous dissipation contribution to turbine loss (temperature ratio of 2). 
is raised from 1 to 2 . This effect is due to the dissipation coefficient itself changing and not due to a change in the reheat factor. This is discussed more in Chapter 7. The viscous component of the exergy loss coefficient rises by $24.4 \%$ as the mainstream-to-coolant temperature ratio is raised from 1 to 2 . This leads to an $11.2 \%$ difference between viscous components of loss coefficient at a temperature ratio of 2 . This difference is solely due to effect of heat transfer on the value of heat shown in Figure 5.6. The drop in temperature near the wall results in a drop in the Carnot cycle efficiency and consequently raises the viscous entropy production close to the wall.

This section showed that viscous dissipation in cooled turbines has a different impact on the euergy and exergy methods. This is because the increase in internal energy due to viscous reheat is valued differently. The value placed on heat by the euergy method is based upon pressure and is insensitive to heat transfer. The value placed on heat by the exergy method is based upon temperature and is sensitive to heat transfer. This is an important finding as it demonstrates than an exergy-based approach to performance analysis is not able to predict how heat transfer affects turbine efficiency.

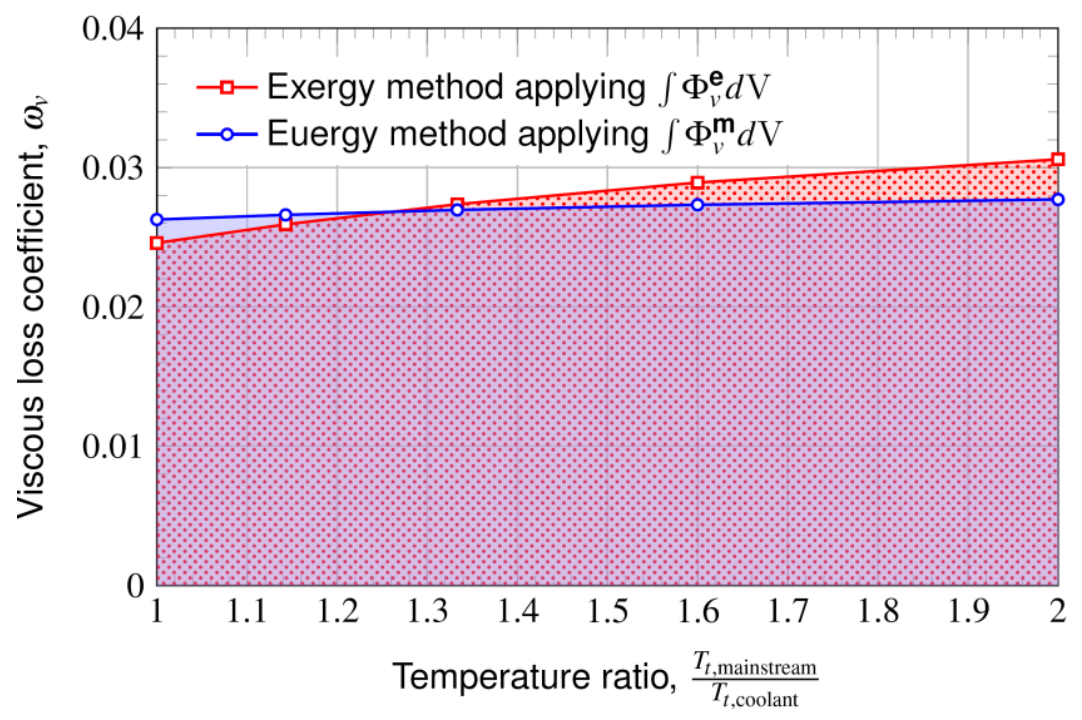

Figure 5.8 - The variation of exergy and euergy viscous loss coefficients with mainstream-to-coolant temperature ratio. 


\subsection{Viscous dissipation}

So far in the chapter, the effect of heat transfer on turbine performance has been attributed to the value placed on heat relative to work. However, heat transfer also acts to change the aerodynamics of the flow. This aerothermal coupling occurs as the temperature gradients cause gradients in thermofluid properties that act to change the amount of dissipation occurring in the boundary layer. More detail on the effect of heat transfer on the boundary layer dissipation coefficient is discussed in Chapter 7. In this section, a RANS solver using the Spalart \& Allmaras (1992) turbulence model is used to investigate this effect.

The effects of temperature dependent fluid properties are modelled in CFD and examined using two sets of simulations. In the first, the variation in viscosity with temperature is set by Sutherland's law, the variation in conductivity with temperature is set using kinetic theory and the density was modelled as an ideal gas. In the second set of solutions, the viscosity and conductivity were set to a constant value equal to that of the mainstream inlet flow while the density was still modelled as an ideal gas.

The first point to note is the change of conductivity had a negligible effect on boundary layer dissipation coefficient. The second is the effect of density change and viscosity change had opposing effects on the dissipation coefficient. The variation of density and viscosity through the boundary layer and its effect on viscous dissipation are shown in Figure 5.9.

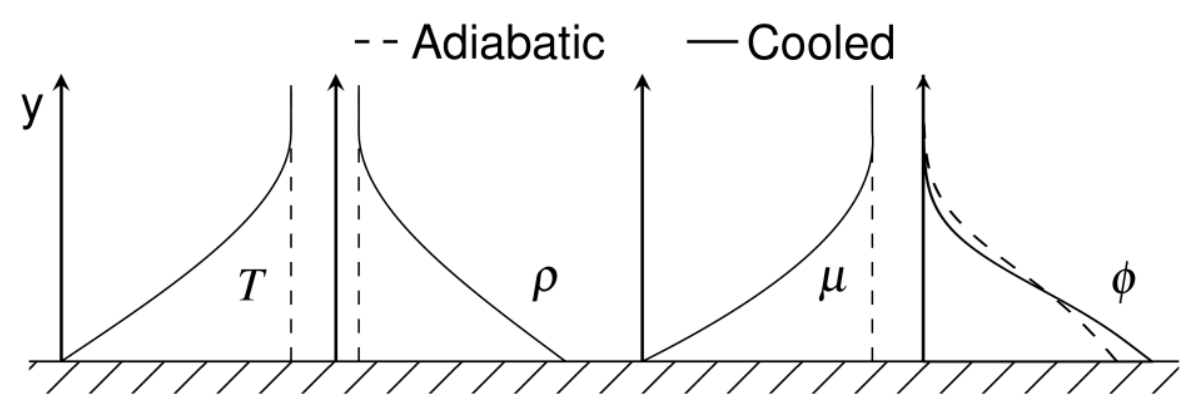

Figure 5.9 - Profiles of temperature, density, viscosity and viscous dissipation for a cooled boundary layer. 


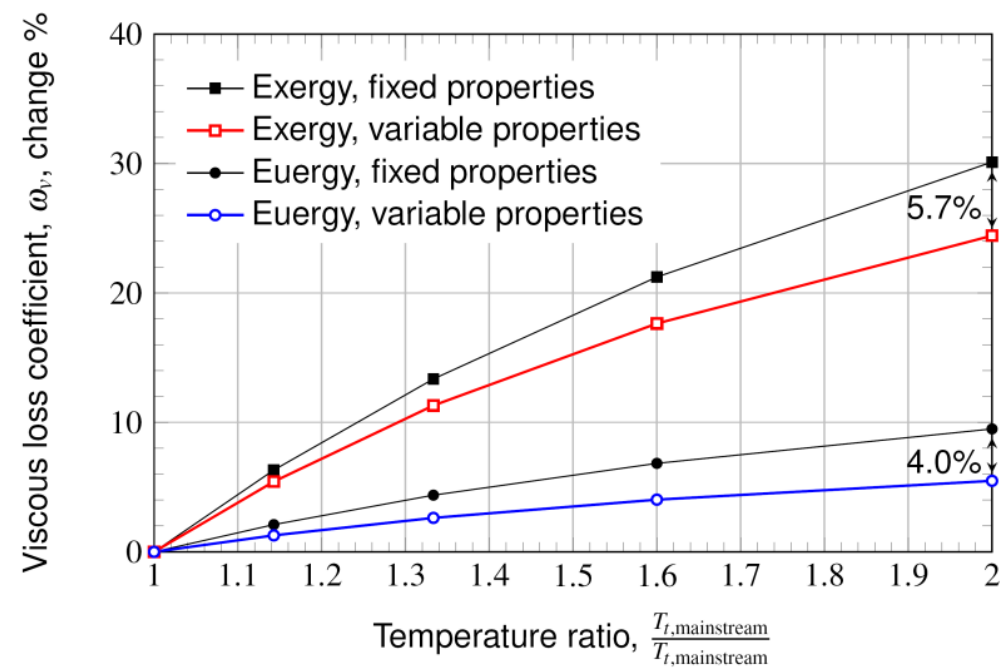

Figure 5.10 - The relative change in viscous loss coefficient with mainstream-to-coolant temperature ratio for fixed and variable thermofluid property simulations.

The change in viscous loss coefficient with mainstream-to-coolant temperature ratio for these two sets of simulations is shown in Figure 5.10.

Using the euergy method, a $4.0 \%$ difference in viscous loss coefficient is predicted at a temperature ratio of 2 between the fixed and variable property simulations. For fixed properties, the loss due to viscous dissipation increases by $9.5 \%$ as the temperature ratio was varied from 1 to 2 . This rise is due to the change in density. For variable properties, the loss due to viscous dissipation increases by $5.5 \%$ as the temperature ratio was varied from 1 to 2 . This rise is lower due to the partial cancellation of the effect of varying density and varying viscosity.

Using the exergy method, a 5.7\% difference in viscous loss coefficient is predicted at a temperature ratio of 2 between the fixed and variable property simulations. This change in sensitivity relative to the euergy method stems from the distribution of viscous dissipation through the boundary layer. As discussed in the previous section, where the viscous dissipation occurs in a cooled boundary layer changes how it impacts the exergy loss coefficient. As heat transfer changes the distribution of viscous dissipation, it also changes its impact on the exergy loss coefficient. However, the same underlying cancellation between the changes in the density and viscosity fields can still be seen. 
This section outlined the effect of heat transfer on the viscous dissipation in a turbine blade row. Heat transfer causes temperature gradients in the flow that result in gradients in thermofluid properties. The effect of density and viscosity variation through the boundary layer, which partially cancel each other, act to change the magnitude and distribution of viscous dissipation. For the euergy method, the increase in magnitude is predicted to raise the viscous loss coefficient by $5.5 \%$. For the exergy method, the altered distribution also changes the sensitivity of the loss coefficient to viscous dissipation as the value of heat varies through the boundary layer. More detail on this effect is discussed in Chapter 7.

\subsection{Summary}

This chapter examined the effect of heat transfer on the loss mechanisms present in a cooled turbine cascade.

First, the effect of heat transfer between streams was discussed. It was shown that the thermal loss mechanism for a general method can be thought of as a form of thermal recuperation; recovering energy from the exhaust that would otherwise be wasted. This happens when heat is transferred across gradients in thermal efficiency. For the exergy method, this occurs when heat is transferred across gradients in temperature. This can only act to increase the exergy loss coefficient and does not represent the effect of heat transfer on turbine performance. For the euergy method, this occurs when heat is transferred across gradients in pressure. This newly discovered loss mechanism was shown to act to reduce the loss coefficient by $7.0 \%$ (between a mainstream-to-coolant temperature ratio of 1 and 2 ) and is not current accounted for in turbine design. By recovering energy from the exhaust, thermal recuperation can be used to improve performance.

Second, the reheat effect due to viscous dissipation was discussed. When viscous dissipation occurs, kinetic energy is converted into internal energy. The reduction in kinetic energy affects the exergy and euergy methods equally, but the increase in internal energy does not. This increase is equivalent to a heat addition, which is valued by the exergy method using the Carnot cycle efficiency and by the euergy method using the Joule cycle efficiency. For a cooled turbine, the profile of Carnot cycle and Joule cycle efficiencies through the boundary 
layer are different, resulting in different values being placed on the increase in internal energy. At a mainstream-to-coolant temperature ratio of 2, viscous dissipation affects the exergy loss coefficient $11.2 \%$ more than the euergy loss coefficient. Even the viscous component of the exergy method is misleading to a cooled turbine designer.

Finally, the effect of heat transfer on viscous dissipation was outlined. The temperature gradients created by heat transfer act to change the thermofluid properties. Variation in density and viscosity both act to change viscous dissipation, but their effects partially cancel. It was shown that heat transfer increases the viscous dissipation by $5.5 \%$ between a mainstream-tocoolant temperature ratio of 1 and 2. This complex coupling is further discussed in Chapter 7 . By accurately modelling this effect, the best aerothermal performance can be achieved. 



\section{Chapter 6 Modelling loss mechanisms in cooled}

\section{turbines}

By accurately calculating turbine performance, informed decisions can be made when trading between different component or cycle designs. Many of these decisions are taken early in the design process. The preliminary design stage is reliant upon simplified models that have distilled complex flow features into their effect on performance. This chapter aims to develop simple predictive models that capture the effect of heat transfer on turbine performance. The euergy method is applied to demonstrate how these models can be compared to well-resolved conjugate CFD simulations of a turbine cascade.

In the cascade, the turbine blade has the same profile as the internally-cooled blade analysed earlier, but now 5 cylindrical cooling holes have been drilled through the surface to provide film cooling. The cascade has been divided into smaller sub-volumes so the loss predicted by CFD can be compared to the simplified models. These loss models are discussed in two sections:

First, the effect of thermal creation is modelled. This was achieved by splitting the loss into contributions either within the flow or across the wall. Only the contribution across the wall was modelled as this accounts for most of the loss.

Second, the effect of viscous dissipation is modelled. This was achieved by splitting the loss into contributions from the boundary layers, cooling holes and trailing edge. 


\subsection{Modelling thermal creation}

The effect of thermal creation is to enable a turbine to extract work from energy that would otherwise be wasted in the exhaust. This is a type of thermal recuperation that occurs when heat is transferred across differences in Joule cycle efficiency.

Figure 6.1 shows the effect of thermal creation in the flow around the film-cooled turbine blade. This is greatest close to the suction-side surface as there is relatively high rates of heat transfer and a relatively low radius of curvature. However, this only accounts for $10 \%$ of the total thermal creation. The remaining $90 \%$ occurs due to heat transfer across the blade walls.

A model has been developed to capture the dominant effect of thermal creation that occurs across the blade walls.

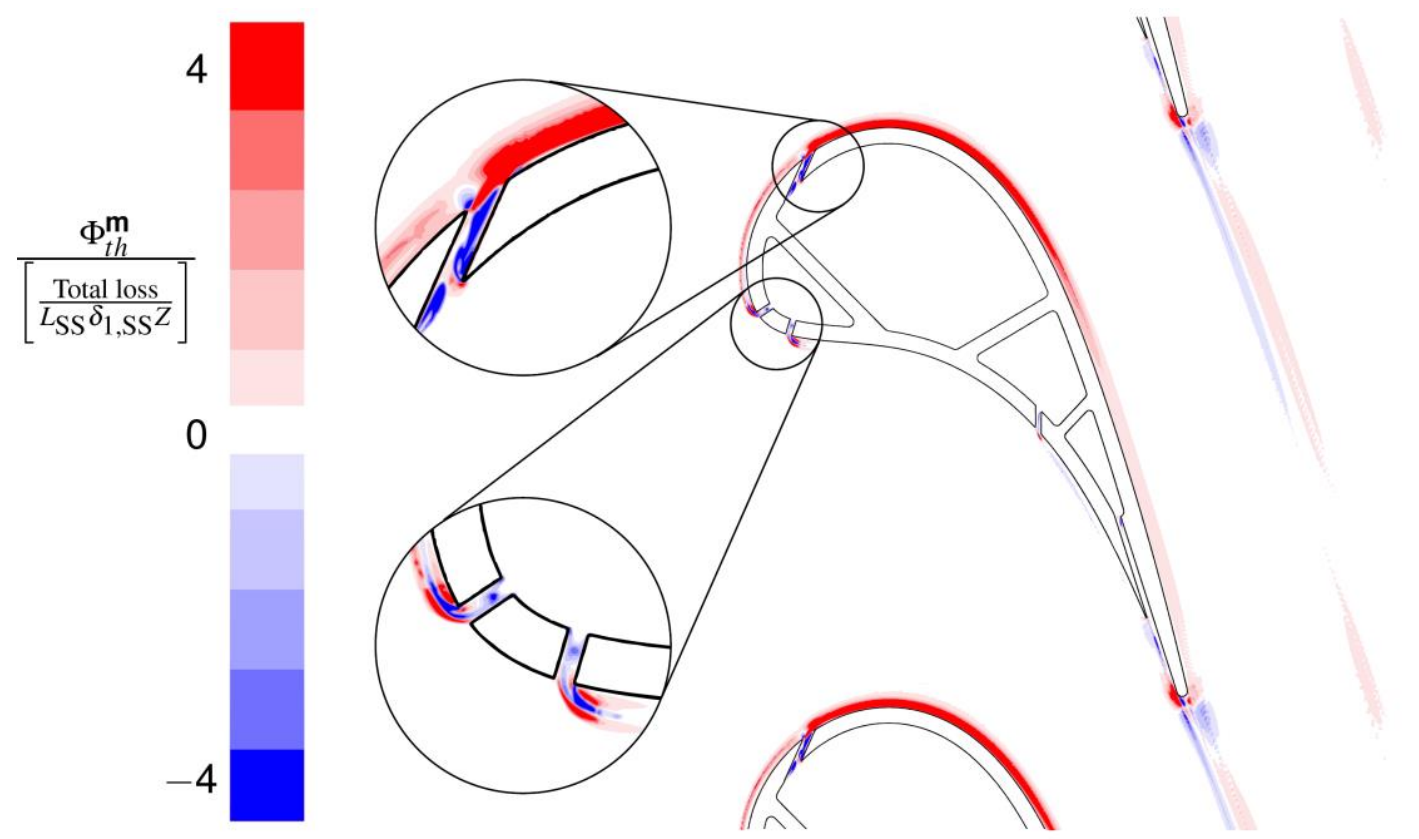

Figure 6.1 - Contours of the effect of thermal creation for the film-cooled blade (mainstream-to-coolant temperature ratio of 2). 


\subsubsection{Heat transfer across the turbine wall}

This model considers heat transfer between the Joule cycle efficiency of the mainstream and coolant stream. The local Joule cycle efficiency depends on the static pressure of the flow. As the variation of static pressure in the coolant streams is very small compared to the mainstream, the Joule cycle efficiency of each coolant stream can be considered constant. Assuming onedimensional conduction, this allows the effect of thermal creation to be modelled by integrating heat flux around the external blade surface only. The formula for this model is given by Equation 6.1.

$$
\omega_{\text {th }}^{\mathbf{m}} \approx \frac{\iint_{S_{\text {blade }}}\left[\left(\frac{p_{0}}{p_{c}}\right)^{\frac{\gamma-1}{\gamma}}-\left(\frac{p_{0}}{p_{m}}\right)^{\frac{\gamma-1}{\gamma}}\right] \boldsymbol{q} \cdot d S}{\iint_{S_{\text {out }}} \rho \boldsymbol{V} \frac{V^{2}}{2} d S}
$$

\subsubsection{Thermal model and CFD comparison}

A comparison between this model and the effect of thermal creation predicted in the conjugate CFD simulation is shown in Figure 6.2. The agreement is good with the model estimating the thermal creation term with an error of $14 \%$. Overall, there is an error of $3.4 \%$ due to the partial cancellation of errors in the flow and across the wall.

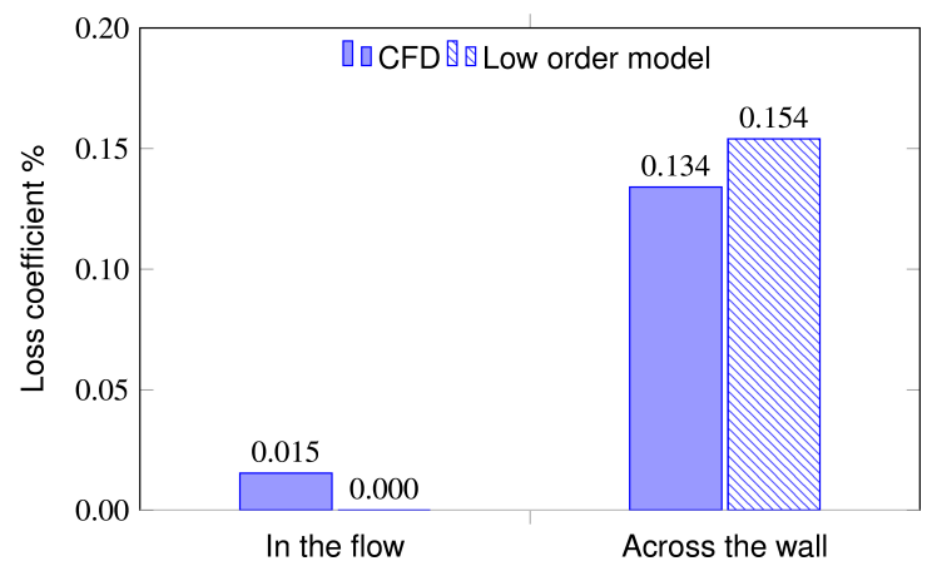

Figure 6.2 - Decomposition of the effect of thermal creation on the loss coefficient of a film-cooled blade. 


\subsection{Modelling viscous dissipation}

The effect of viscous dissipation is to reduce the work that a turbine could extract by converting kinetic energy into internal energy. A reversible adiabatic turbine could extract all the kinetic energy, but only a fraction of the internal energy.

Figure 6.3 shows the effect of viscous dissipation in the flow around a film-cooled turbine blade. This shows the greatest effect is along the suction-side boundary layer, near the coolant holes and in the downstream wake region. Unlike thermal creation, all viscous dissipation occurs in the flow.

Three models have been developed to capture the effect of viscous dissipation occurring in the flow around the turbine blade. The models consider the causes of viscous dissipation in two control volume shown in Figure 6.4. In the control volume around the blade, $\mathrm{CV}_{1}$, viscous dissipation occurs due to shear stresses acting through the boundary layer or due to viscous mixing of streams as the coolant flow is introduced into the mainstream. In the control volume downstream of the blade, $\mathrm{CV}_{2}$, viscous dissipation occurs due to viscous mixing of the wake. Each of these three viscous processes are modelled: boundary layer dissipation, $\omega_{B L}^{\mathrm{m}}$, cooling

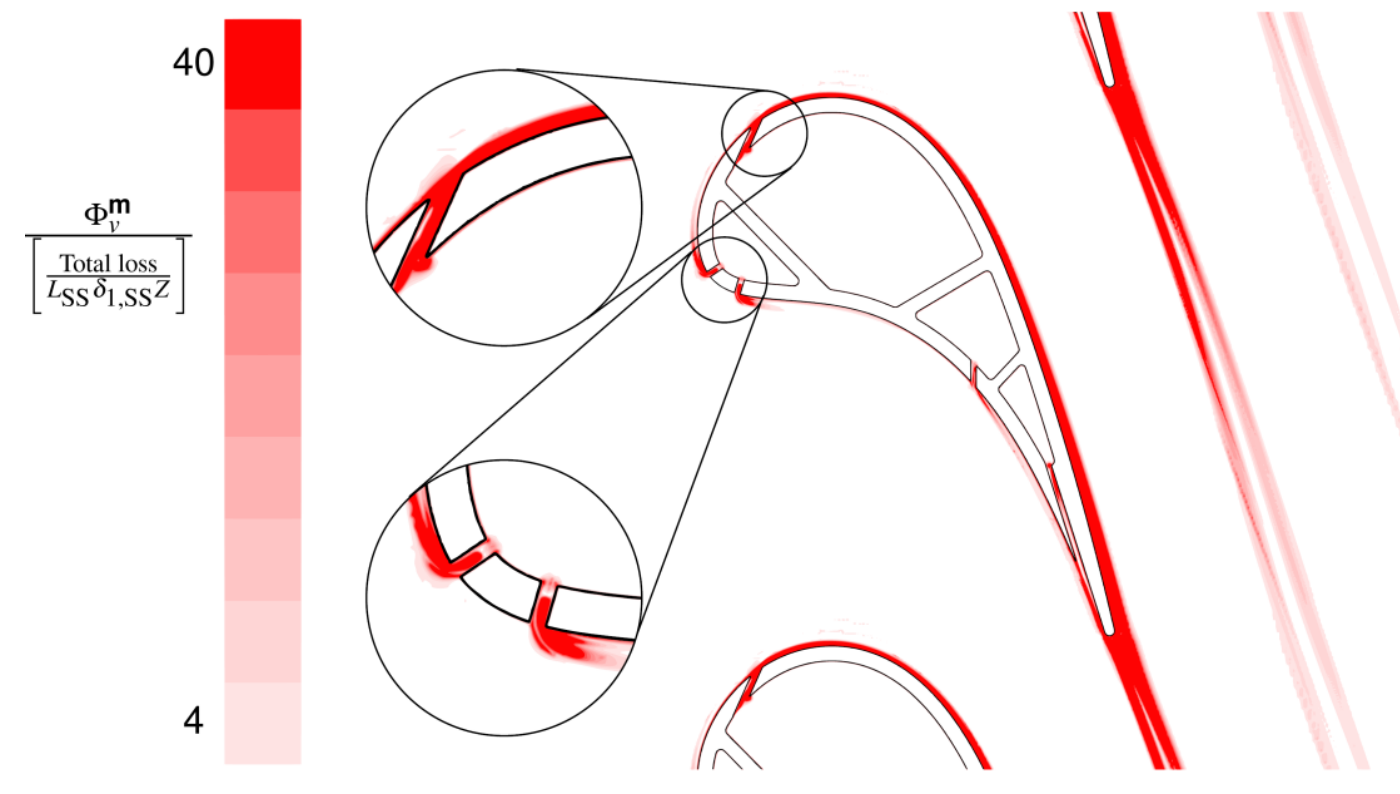

Figure 6.3 - Contours of the effect of viscous dissipation for the film-cooled blade (mainstream-to-coolant temperature ratio of 2). 


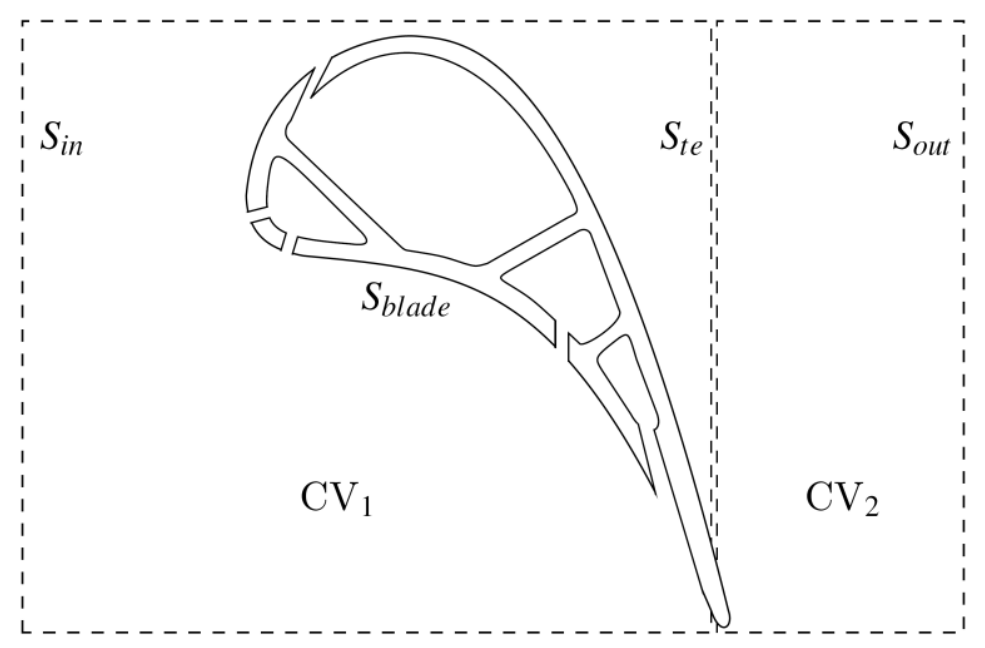

Figure 6.4 - The turbine loss coefficient divided into a control volume around the blade, $\mathrm{CV}_{1}$, and a control volume downstream of the trailing edge, $\mathrm{CV}_{2}$.

hole mixing, $\omega_{C H}^{\mathrm{m}}$, and trailing edge mixing, $\omega_{T E}^{\mathrm{m}}$. Equation 6.2 demonstrates the loss coefficient decomposition.

$$
\omega_{v}^{\mathrm{m}}=\frac{\iiint_{\mathrm{CV}_{1}}\left(\frac{p_{0}}{p}\right)^{\frac{\gamma-1}{\gamma}} \phi d \mathrm{~V}}{\iint_{S_{\text {out }}} \rho \boldsymbol{V} \frac{V^{2}}{2} d S}+\frac{\iiint_{\mathrm{CV}_{2}}\left(\frac{p_{0}}{p}\right)^{\frac{\gamma-1}{\gamma}} \phi d \mathrm{~V}}{\iint_{S_{\text {out }}} \rho \boldsymbol{V} \frac{V^{2}}{2} d S} \approx\left(\omega_{B L}^{\mathrm{m}}+\omega_{C H}^{\mathrm{m}}\right)+\omega_{T E}^{\mathrm{m}}
$$

\subsubsection{Boundary layers}

The loss model for the boundary layer flow utilises a dissipation coefficient, $c_{d}$, as shown in Equation 6.3. Schlichting (1968) presents this coefficient to normalise the dissipation in the boundary layer. Denton (1993) applies this coefficient turbomachinery flow using the exergy method and suggested that $c_{d} \approx 0.002$. In this chapter, this value has been adopted for clarity and the euergy method has been applied to extend this model to cooled turbines. Better agreement can be achieved using a more accurate boundary layer dissipation coefficient.

$$
\omega_{B L}^{\mathrm{m}}=\frac{\iint_{S_{\text {blade }}}\left(\frac{p_{0}}{p}\right)^{\frac{\gamma-1}{\gamma}} c_{d} \rho_{m} V_{m}^{3} d S}{\iint_{S_{\text {out }}} \rho \boldsymbol{V} \frac{V^{2}}{2} d S}
$$




\subsubsection{Cooling holes}

The loss model for the flow out of the cooling holes simply considers the change in kinetic energy of the coolant. Hartsell (1972) first presented the basis of the model which was then extended by Young \& Wilcock (2002) for the exergy method. Here, the euergy method has been applied to update the value based on heat and is shown in Equation 6.4.

The first term represents the component of velocity tangential to the surface and the second term represents the normal component. It was assumed that the coolant equilibrates to the mainstream velocity tangential to the surface. It was also assumed that all the kinetic energy of the normal velocity component is dissipated. Better agreement could be achieved by modelling this mixing process more accurately.

$$
\omega_{C H}^{\mathrm{m}}=\frac{\sum\left(\frac{p_{0}}{p}\right)^{\frac{\gamma-1}{\gamma}} \delta m_{c}\left[\frac{\left(V_{m}-V_{c} \cos \alpha\right)^{2}}{2}+\frac{\left(V_{c} \sin \alpha\right)^{2}}{2}\right]}{\iint_{S_{\text {out }}} \rho \boldsymbol{V} \frac{V^{2}}{2} d S}
$$

\subsubsection{Trailing edge}

Denton \& Xu (1989) considered the change in stagnation pressure coefficient downstream of transonic turbines. Denton (1993) decomposed the change in stagnation pressure coefficient into aerodynamic design parameters for incompressible flow, shown in Equation 6.5.

$$
\frac{p_{t, 1}-p_{t, 2}}{0.5 \rho_{t e} V_{t e}^{2} w} \approx \frac{\delta_{2, t e}}{w}-c_{p b} \frac{t}{w}+\left(\frac{t+\delta_{1, t e}}{w}\right)^{2}
$$

When the losses around the blade are subtracted, Equation 6.6 presents the loss that occurs downstream of the trailing edge in $\mathrm{CV}_{2}$.

$$
\omega_{T E}^{p}=\frac{p_{t, t e}-p_{t 2}}{0.5 \rho_{t e} V_{t e}^{2} w} \approx \frac{\delta_{3, t e}}{w}\left[\frac{2}{H_{32, t e}}-1\right]-c_{p b} \frac{t}{w}+\left(\frac{t+\delta_{1, t e}}{w}\right)^{2}
$$


For high speed flows with heat transfer, Chapter 4 demonstrated that the stagnation pressure loss coefficient does not accurately reflect turbomachinery performance. This calls into question the accuracy of this decomposition.

This decomposition can be updated for the euergy loss coefficient. For a cooled turbine, although there is significant heat transfer across the blade surface, the downstream control volume, $\mathrm{CV}_{2}$, is approximately adiabatic. This allows the euergy loss coefficient to be estimated from the change in Mach number and for the euergy loss coefficient to be related to the kinetic energy loss coefficient, as demonstrated in Appendix B.2 and Appendix B.3 respectively.

The kinetic energy loss has been considered previously by Drela (2009) for thin trailing edges. Equation 6.7 extends the kinetic energy loss coefficient for thick trailing edges.

$$
\omega_{T E}^{K E^{\prime}}=\left[1-\frac{V_{o u t}^{2}}{V_{t e}^{2}}\right] \frac{\left(w-t-\delta_{1, t e}\right)}{w}-\frac{\delta_{3, t e}}{w}
$$

This kinetic energy loss coefficient becomes the stagnation pressure loss coefficient under the assumption that the density of the flow is approximately constant (i.e. $\rho_{\text {in }}=\rho_{\text {out }}$ ), shown in Equation 6.8. This is demonstrated in Appendix B.1.

$$
\omega_{T E}^{K E^{\prime}} \approx \frac{\delta_{3, t e}}{w}\left[\frac{2}{H_{32, t e}}-1\right]-c_{p b} \frac{t}{w}+\left(\frac{t+\delta_{1, t e}}{w}\right)^{2}
$$

As the cooled turbine has significant density variation, determining the kinetic energy loss coefficient and relating it to the euergy loss coefficient was appropriate.

\subsubsection{Viscous models and CFD comparison}

The film-cooled turbine cascade has been divided into 13 regions as depicted in Figure 6.5. By integrating these regions in CFD simulations, the accuracy of the models was assessed. Along the pressure-side (PS), suction-side (SS) and the 4 internal passages, the boundary layer model was applied (Equation 6.3). The size and aspect ratio of the cooling hole zones was set based upon the local contours of viscous dissipation around each hole. In the 5 regions across the cooling holes, a combination of the boundary layer model and the cooling hole model were 


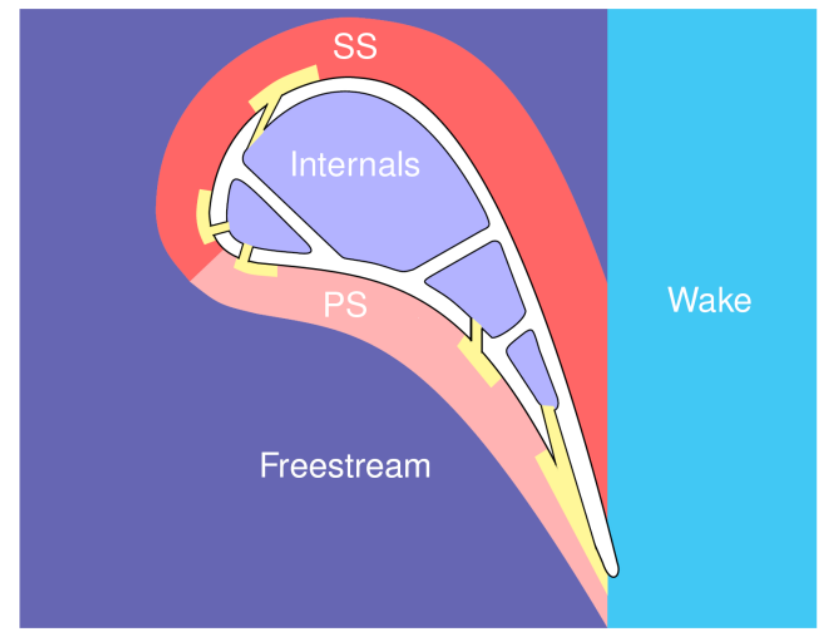

Figure 6.5 - Diagram of the film-cooled turbine blade divided into loss accounting regions.

applied (Equations 6.3 and 6.4). In the wake region, the wake mixing model was applied (Equation 6.7). The freestream was found to have a negligible loss contribution.

A comparison between the effect of viscous dissipation predicted by the three viscous models and the conjugate CFD simulation is shown in Figure 6.6. Using this decomposition, the CFD attributes $64.3 \%$ to the boundary layer, $7.3 \%$ of loss to the cooling holes and $28.4 \%$ to the wake. Overall, the agreement is good with only a $1 \%$ error in the viscous component of blade loss coefficient. The largest contribution to this error along the suction-side boundary layer.

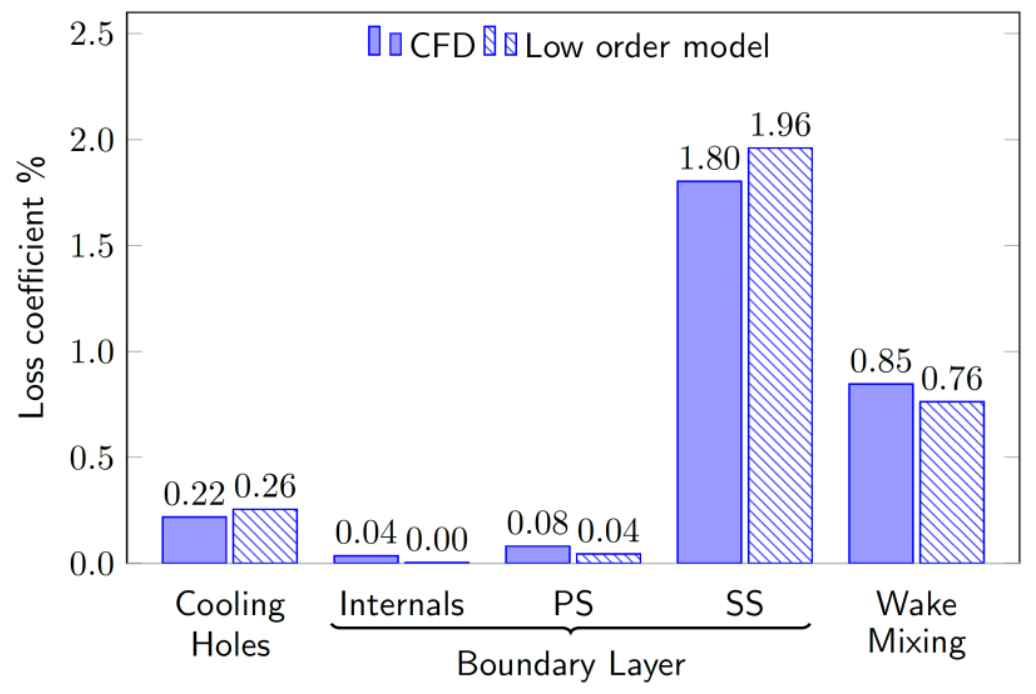

Figure 6.6 - Decomposition of the effect of viscous dissipation on the loss coefficient of a film-cooled blade. 


\subsection{Summary}

This chapter examined the ability of simple models to capture the effect of heat transfer on cooled turbine performance.

Overall, the method of decomposing a flow into zones has proved a simple and robust way examining the loss mechanisms in complex cooled geometries. This is likely to be a powerful and physically correct way of comparing the underlying loss mechanism of new designs concepts. It was found, when compared to the CFD predictions, the loss coefficient estimated by the proposed models agreed to within $1 \%$. The agreement is surprisingly good and masks a variation in the accuracy of individual loss components. These are accurate to within $5.6 \%$ of total loss coefficient. The loss models were developed to capture the effects of thermal creation and viscous dissipation.

First, $90 \%$ of the effect of thermal creation was shown to occur due to heat transfer across the walls of the turbine blade. This can be accurately modelled by considering the heat flux leaving mainstream through the blade surface. This indicates that accurate performance predictions could be achieved by only solving the mainstream flow.

Second, the effect of viscous dissipation was found to be accurately captured by updating the value placed on heat used by current models. The dissipation in the boundary layer flow can be accurately modelled using a dissipation coefficient. The dissipation due to coolant flow being introduced into the mainstream can be accurately modelled using an updated mixing model. The dissipation downstream of the trailing edge can be accurately modelled by updating loss decomposition for the euergy method. As many existing loss models just require updating the value placed on heat, this reduces the barrier for industrial designers to adopt the euergy method to calculate turbine performance. 



\section{Chapter 7 Effect of heat transfer on boundary layer dissipation coefficient}

In turbomachinery flows, the dominant source of loss is due to the dissipation of kinetic energy. This viscous dissipation is particularly great in the flow close to the wall, where there are large shear-stresses. In cooled turbines, the near-wall region also experiences high rates of heat transfer to ensure that the turbine blades survive. The heat transfer through the boundary layer creates temperature gradients which alter the shear-stresses. This chapter investigates how this near-wall coupling between the temperature gradients and viscous dissipation affects turbine performance. There are four sections:

The first section postulates the effect of heat transfer by examining integral boundary layer methods (IBLM). The Von Karman integral equation has been combined with the kinetic energy integral equation to decompose the effect of heat transfer on the dissipation coefficient. This integral approach is useful as it is applicable to both laminar and turbulent flows. This section identifies the effect of heat transfer on boundary layer flow is due to two mechanisms: the change in skin friction and the change in a pressure-coupling term.

The second section examines the effect of heat transfer on the boundary layer dissipation coefficient for a near-zero pressure gradient flat plate. This focuses on examining the link between skin friction and dissipation coefficient for both laminar flow, using a compressible Blasius solution, and turbulent flow, using a combination of RANS and DNS results. Using information about the velocity profiles, the physical mechanism of how heat transfer alters dissipation through the boundary has been determined.

The third section examines the effect of heat transfer on the boundary layer dissipation coefficient for a flat plate subject to a freestream pressure gradient. This focus on examining the coupled effect of heat transfer and pressure gradient for both laminar and turbulent flow. It 
is illustrated that, while current models can capture each effect in isolation, their coupled effect remains unclear.

The final section examines the effect of heat transfer on turbomachinery performance. An internally-cooled turbine rotor blade has been simulated using several RANS models to illustrate the uncertainty in predicting the effect of heat transfer using current design methods.

\subsection{Integral boundary layer methods}

The aim of this section is to outline a framework to examine the effect of heat transfer on dissipation coefficient. The integral boundary layer method presented stems from combining conservation of momentum and the transport of kinetic energy so is applicable to a wide variety of flows. This allows a unified view of the effect of heat transfer on both laminar and turbulent flow.

When the wall is cooled, many boundary layer integral properties change. This is primarily because many integral properties integrate the density profile which is strongly affected by cooling. The objective was to relate compressible boundary layer integral properties to an incompressible form.

This has been achieved by Standen (1964) and has been extended here to include the energy shape factor $H_{32}$. The derivation first uses a modified Stewartson transformation to absorb the density distribution into the length-scale. For any quantities still reliant on the temperature profile, the Van Driest profile was used to relate temperature to velocity. This allows compressible and incompressible forms to be related but relies on the link between the temperature and velocity profile to hold (i.e. forced convection with small temperature and pressure gradients). By substituting these relations into the energy-integral equation presented by Schlichting (1968), Equation 7.1 is found.

$$
c_{d}=\underbrace{\frac{c_{f}}{2} \frac{H_{32, i}}{2}}_{\text {Flat plate }}-\underbrace{\left(\left(\frac{T_{w}}{T_{m}}-\frac{T_{a w}}{T_{m}}\right)-\frac{H_{32, i}}{2}\left(\frac{T_{w}}{T_{m}} H_{12, i}-\frac{T_{a w}}{T_{m}}\right)\right)}_{\text {Pressure gradient coupling }} \Lambda+\underbrace{\frac{\delta_{2}}{2} \frac{\partial H_{32, i}}{\partial x}}_{\approx 0}
$$


The dissipation coefficient has been decomposed into three terms. The first term is the direct effect of skin friction and is the only non-zero term for a zero pressure-gradient equilibrium boundary layer. The second term is the influence of pressure gradients and is scaled by the influence of heat transfer. The final term is due to the change in energy shape factor which is approximately zero for an equilibrium boundary layer.

To conclude, the effect of heat transfer affects dissipation coefficient in two ways. For flat plate flow, the effect of heat transfer is solely due to the change in skin friction. For flows with a mainstream pressure gradient, heat transfer acts to change the pressure gradient coupling.

\subsection{Linking dissipation coefficient and skin friction coefficient}

For a flat plate, the integral boundary layer method suggests the effect of heat transfer on dissipation coefficient is captured by the change in skin friction. This section aims to identify the physical mechanisms behind how heat transfer changes dissipation coefficient for zero pressure-gradient flows. It is divided into two parts which consider laminar and turbulent flow respectively.

\subsubsection{Laminar boundary layer}

For laminar flow, the effect of heat transfer can be examined using a compressible Blasius solution. The derivation extends the framework presented by Anderson (1989) to consider dissipation (summarised in Appendix C.2). Although this approach makes assumptions concerning the nature of the aerothermal coupling (discussed in Chapter 3), the key advantage is the analytical insight it offers.

Figure 7.1 demonstrates how the effect of heat transfer on both the dissipation coefficient and skin friction by changing the mainstream-to-wall temperature ratio. It clearly shows that the effect of heat transfer on skin friction is reflected by a change in dissipation coefficient. 

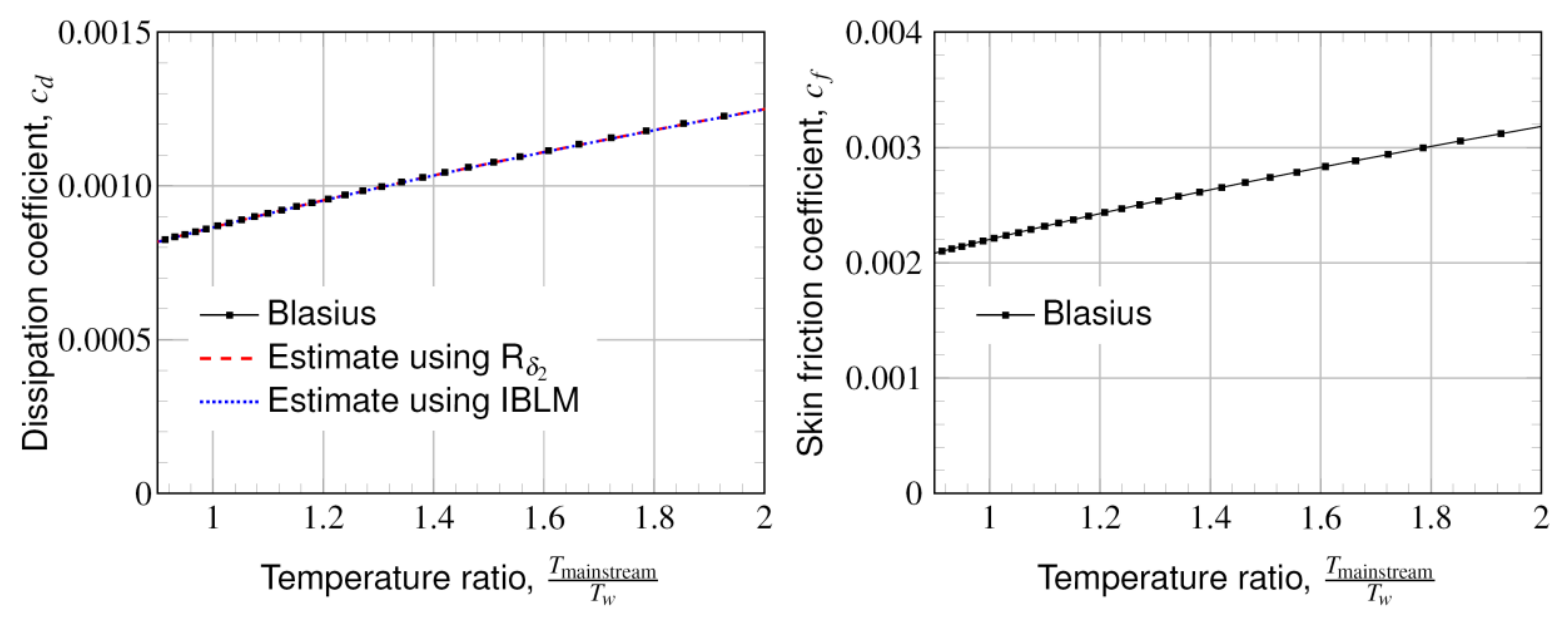

Figure 7.1 - The variation in dissipation coefficient (left) and skin friction coefficient (right) with mainstream-to-wall temperature ratio for laminar flow at $R_{\delta_{2}}=400$.

The change dissipation coefficient due to heat transfer can be modelled in two ways. The first is by rescaling the boundary layer using an appropriate change in Reynolds number. This approach derives from the scaling analysis proposed by Walz (1967). The second approach is to use the estimated integral boundary layer methods. It is worth noting that this approach requires accurate knowledge of skin friction. Both ways capture the effect of heat transfer and obtain good agreement with the Blasius solution (within 0.4\%).

The dissipation coefficient can also be obtained analytically. For a zero pressuregradient boundary layer, the dissipation coefficient can be written as follows:

$$
c_{d}=0.173 \frac{C_{w}}{\mathrm{R}_{\delta_{2}}}
$$

where $C_{w}$ is the Chapman-Rubesin parameter used to describe how viscosity depends on temperature.

Equation 7.2 illustrates that the effect of heat transfer is due to the change in thermofluid properties. These act to change the appropriate Reynolds number that describes the boundary layer, which is accurately captured by both models based upon a change of length-scale and integral boundary layer methods. 


\subsubsection{Turbulent boundary layer}

For turbulent flow, the effect of heat transfer can be examined using numerical techniques. To capture the correct effect, it was necessary to generate high-fidelity solutions of the flow. As they are computationally expensive, only this section contains DNS data which focusses on the effect of heat transfer on a near-zero pressure-gradient flat plate. This canonical case is compared to a range of RANS models that are typically applied in turbomachinery design.

The high-fidelity simulations used 3DNS (shown in black) while a combination of RANS models were applied in ANSYS Fluent (shown in blue) and TEXSTAN (shown in red). All the data has been taken at $R_{\delta_{2}}=1000$. This allows the behaviour of the underlying physical mechanisms to be examined as well as the ability of current design modelling methods to capture to them.

Figure 7.2 presents the effect of varying wall temperature on the dissipation coefficient (left) and skin friction (right). The DNS results are indicated by the black circles where the white and black centres represent an adiabatic or isothermal wall boundary condition respectively. The error bars represent the difference in dissipation coefficient between using the computed value of turbulent kinetic energy production or dissipation. This error is due to
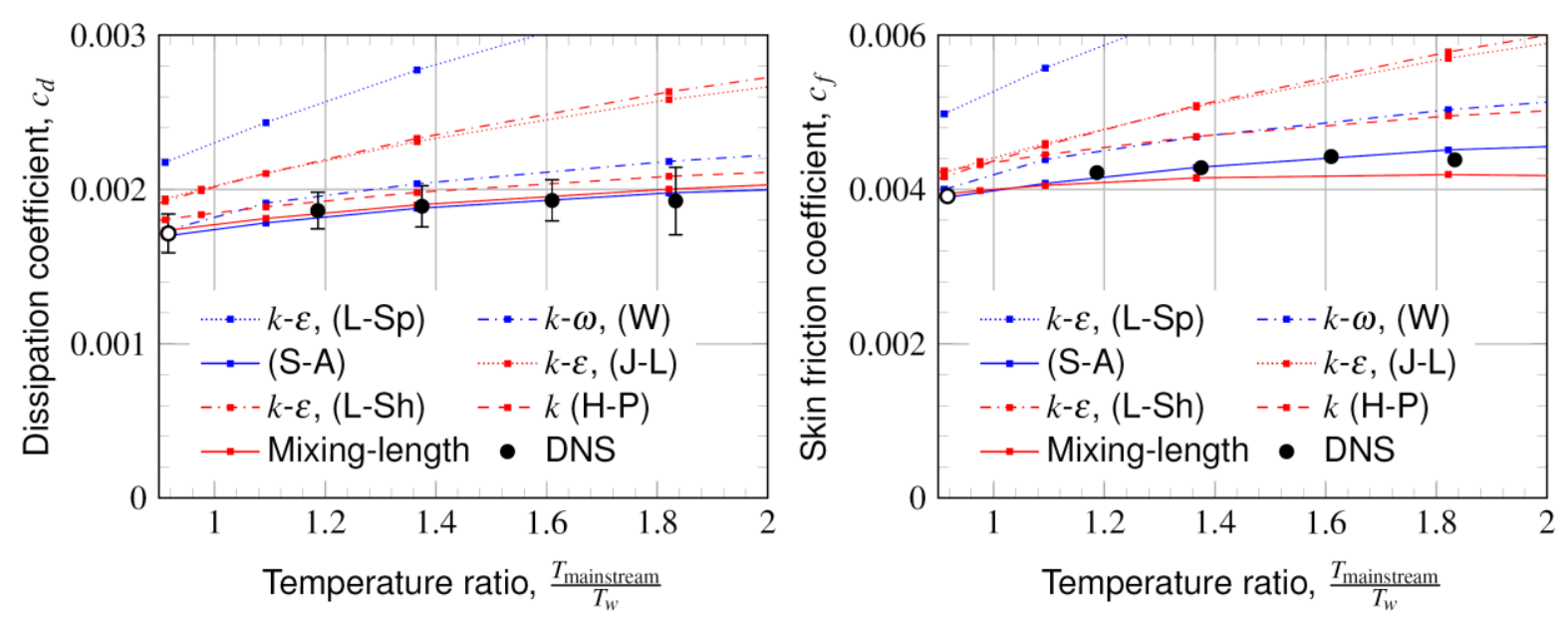

Figure 7.2 - The variation in dissipation coefficient (left) and skin friction coefficient (right) with mainstream-to-wall temperature ratio for turbulent flow at $\mathrm{R}_{\delta_{2}}=1000$.

ANSYS Fluent, blue; TEXSTAN, red; 3DNS, black; hollow marks, adiabatic; filled marks, isothermal. 
the mesh resolution not being sufficient to resolve all the dissipative scales. The lines all represent simulations using different RANS models. The simulations from ANSYS Fluent are in red while those from TEXSTAN are in blue.

By cooling the boundary layer, both the skin friction and dissipation coefficients rise. The mixing-length model, one-equation Hassid \& Poreh (1975) model and Spalart \& Allmaras (1992) model achieve the best agreement with the $k-\omega$ model by Wilcox (1988) just outside numerical uncertainty. However, the $k-\epsilon$ models all struggle to accurately predict the skin friction or dissipation coefficients.

The difficulty of using $k-\epsilon$ models to accurately predict near-wall heat transfer has been well documented. As these models are so widely applied in practical design, they have been retained in this chapter to examine whether they predict the correct behaviour even though the predicted value is less accurate.

Comparing the coefficients in Figure 7.2, the change in skin friction coefficient is mirrored by a change in dissipation coefficient. Applying the integral boundary layer method, the balance between the skin friction and dissipation coefficients is expressed in Equation 7.3.

$$
\frac{2 c_{d}}{c_{f}}=\frac{H_{32}}{2}
$$

This balance is also depicted graphically in Figure 7.3. There is broad agreement with all models achieving a balance within $4 \%$, except the mixing-length model. For the RANS models, the agreement reflects the ability of the solver and model to balance the conservation equations. The mixing-length model in TEXSTAN achieved accurate predictions, but the accuracy of the balance decreases with temperature ratio.

The energy shape factor was predicted by all the simulations to not only have a similar magnitude, but also to be insensitive to heat transfer. This agrees with the analytical prediction from the previous section. This confirms that, for a flat plate, the change in dissipation coefficient is proportional to the change skin friction. 

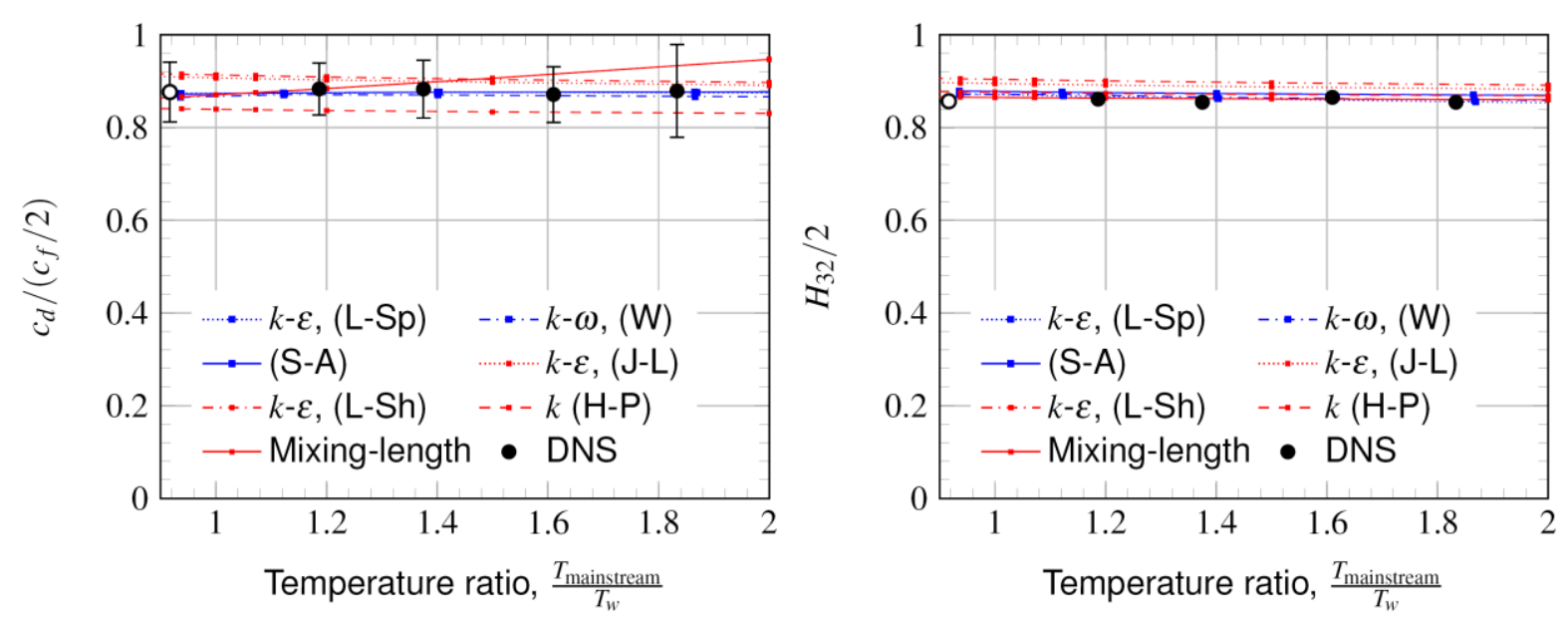

Figure 7.3 - The variation in the ratio of dissipation and skin friction coefficients (left) and energy shape factor (right) with mainstream-to-wall temperature ratio for turbulent flow at $\mathrm{R}_{\delta_{2}}=1000$.

ANSYS Fluent, blue; TEXSTAN, red; 3DNS, black; hollow marks, adiabatic; filled marks, isothermal.

In turbulent flow, the dissipation coefficient can be decomposed into two contributions; one due to the mean velocity distribution and the other due to the fluctuations in velocity. The dissipation coefficient is defined in this thesis as the sum of the effect of mean dissipation, $\Phi$, and the effect of the production of turbulent kinetic energy (TKE), П, given in Equation 7.4.

$$
c_{d}=\underbrace{\int \frac{\tau}{\rho_{m} u_{m}^{3}} \frac{\partial \bar{u}}{\partial y} d y}_{\Phi}+\underbrace{\int \frac{-\overline{u v}}{\rho_{m} u_{m}^{3}} \frac{\partial \bar{u}}{\partial y} d y}_{\Pi}
$$

This decomposition is shown in Figure 7.4 with the effect of mean dissipation on the left and the effect of the production of TKE on the right. Most of the effect of heat transfer on dissipation coefficient appears to stem from the change in the production of TKE. This is more difficult to capture for the turbulence model resulting in the large spread of predicted values. For heat transfer, this may appear a slightly unexpected result as both heat transfer and the mean dissipation have the greatest effect close to the wall. However, it appears heat transfer has a much greater effect on the production of TKE.

This part has shown that heat transfer affects skin friction and dissipation coefficients in the same way. It has also shown that current turbulence models have differing abilities to capture the effect of heat transfer which mostly stems from their ability to capture the 

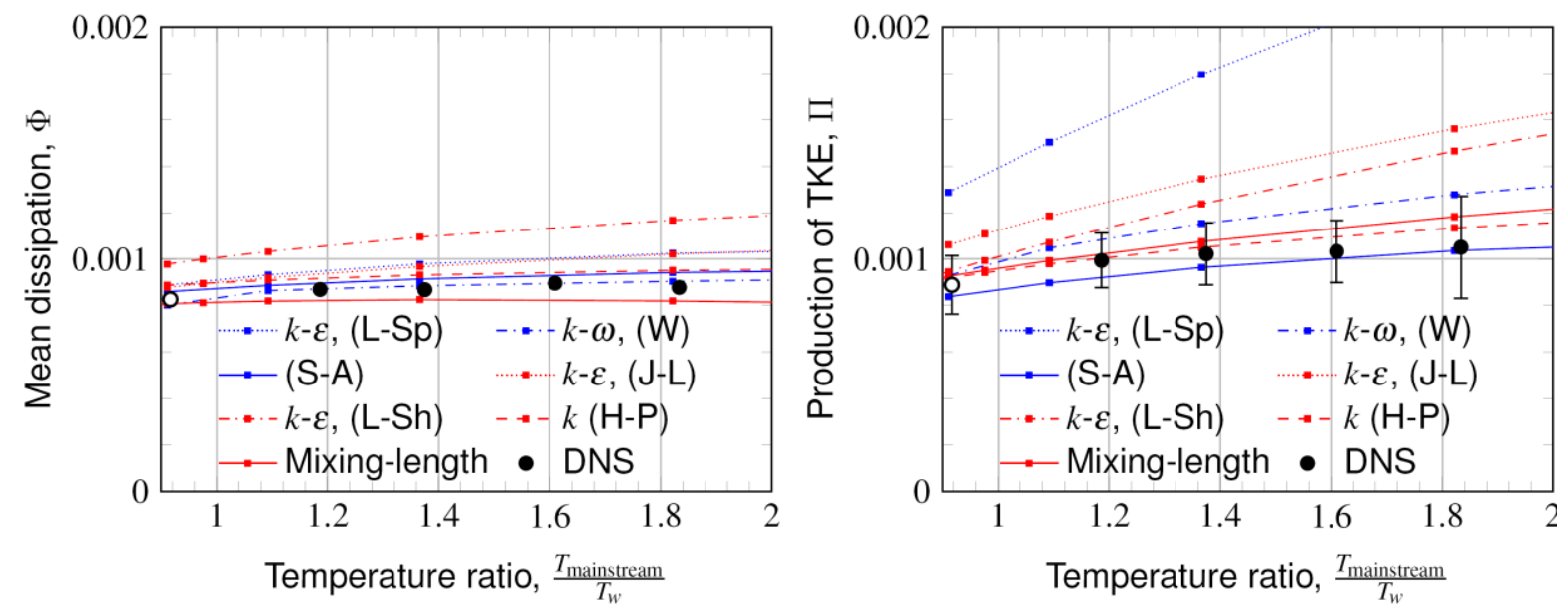

Figure 7.4 - The variation in component of dissipation coefficient due to mean (left) and fluctuating (right) velocities with mainstream-to-wall temperature ratio for turbulent flow at $R_{\delta_{2}}=1000$.

ANSYS Fluent, blue; TEXSTAN, red; 3DNS, black; hollow marks, adiabatic; filled marks, isothermal.

production of TKE. In the next part, the DNS results will be used to examine the underlying physical mechanism behind how heat transfer affects turbine performance. Then, this understanding will be used to explain why heat transfer has a greater effect on the production of TKE than on the mean dissipation.

\subsubsection{The effect of heat transfer on the dissipation profile}

How heat transfer affects dissipation coefficient can be examined using velocity profiles. For laminar flow, this was relatively straight-forward as a clear analytical velocity profile exists. For turbulent flow, there is no consensus on how to best normalise a compressible velocity profile.

The traditional approach to normalising a velocity profile into inner units is to use the local density and viscosity. A recent approach, developed by Trettel \& Larsson (2016), is based upon semi-local scaling (SL). This method attempts to correct for the rescaling of the boundary layer due to changes in density and viscosity. The two methods are shown in Equation 7.5. 


$$
U^{+}=\int_{0}^{u^{+}} d u^{+}, \quad U_{S L}^{+}=\int_{0}^{u^{+}}\left(\frac{\bar{\rho}}{\rho_{w}}\right)^{\frac{1}{2}}\left[1+\frac{1}{2} \frac{1}{\bar{\rho}} \frac{d \bar{\rho}}{d y} y-\frac{1}{\bar{\mu}} \frac{d \bar{\mu}}{d y} y\right] d u^{+}
$$

The ability of these two methods to normalise the DNS velocity distributions are shown in Figure 7.5. The variable $\Omega$ represents the static temperature ratio between the mainstream and wall. The traditional and semi-local methods have been used on the left and right respectively. The semi-local method demonstrates improved similarity between profiles particularly in the buffer and logarithmic region. Normalising by inner units, the dissipation coefficient can be written as:

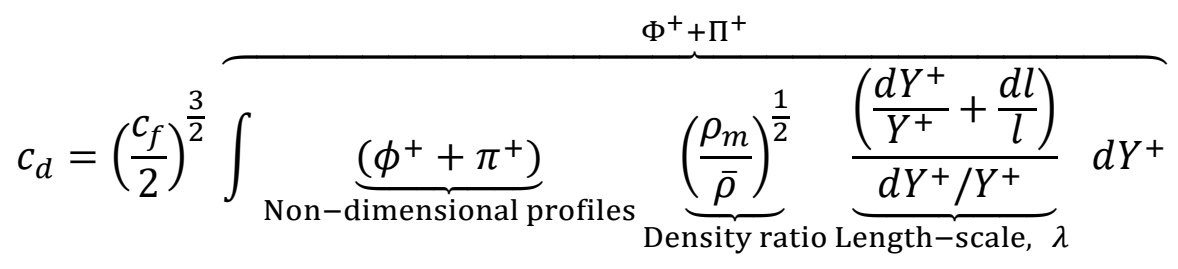

The dissipation coefficient is still obtained by integrating the shear-stress through the boundary layer, but now this has been expressed in inner units. To obtain Equation 7.6, only the $Y^{+}$scaling and knowledge that non-dimensional profiles exist are required. Although the semi-local scaling provides a method to obtain these profiles, this is not used in the following analysis.
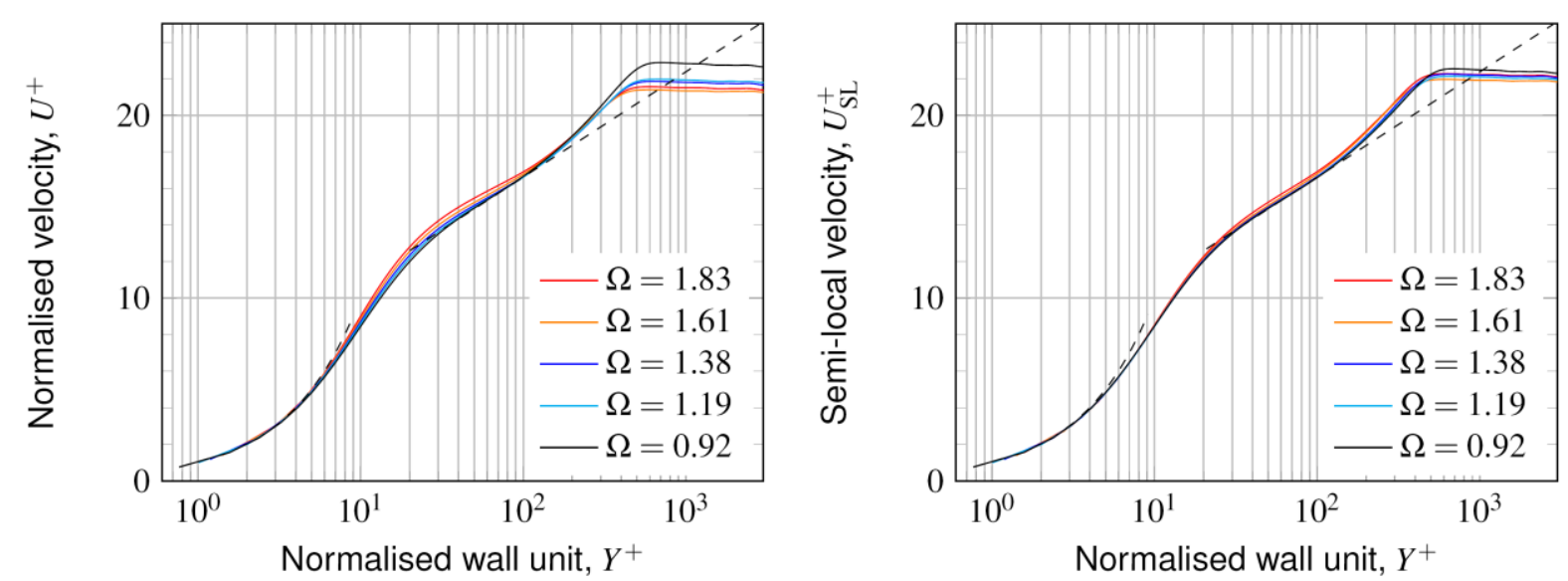

Figure 7.5 - DNS normalised velocity profiles; traditional approach (left), semi-local scaling (right). 
Equation 7.6 indicates that there are three contributions to the dissipation profile. The first term is from the non-dimensional profiles. The following results requires there to be some inner unit scaling to produce self-similar profiles. The second term is a scaling due to the density variation. The third term is the change in turbulent length-scale due to compressibility. Combined, the density and length-scale variation act to alter the dissipation profile.

\section{Non-dimensional profile}

In the DNS, self-similar profiles of boundary layer dissipation can be found by normalising the mean dissipation and production of TKE. These profiles can also be obtained from the velocity gradient based in inner units.

$$
\phi^{+}=\left(\frac{d U^{+}}{d Y^{+}}\right)^{2}, \quad \pi^{+}=-R_{v y}^{+} \frac{d U^{+}}{d Y^{+}}
$$

The local mean dissipation profile, $\phi^{+}$, and local production of TKE profile, $\pi^{+}$, are expressed in Equation 7.7 and shown on the left and right of Figure 7.6 respectively. For all the DNS, a good agreement is obtained between the normalised profiles. This shows that at least an approximately self-similar solution exists. The profile of mean dissipation occurs close to the wall. The contribution above a $Y^{+}$of 10 is small. The profile of production of TKE peaks away from the wall at a $Y^{+}$approximately equal to 10 . The contribution both close to the wall and in the freestream are small.
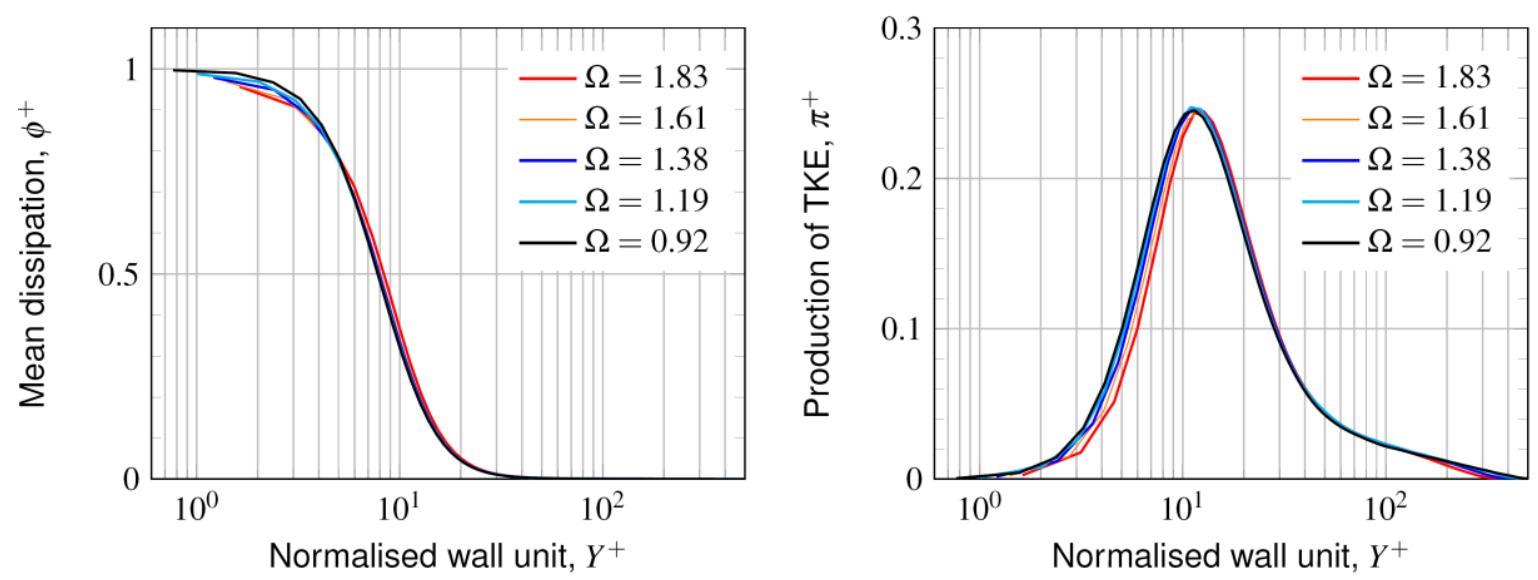

Figure 7.6 - DNS normalised dissipation profiles for mean dissipation and the production of turbulent kinetic energy (TKE). 


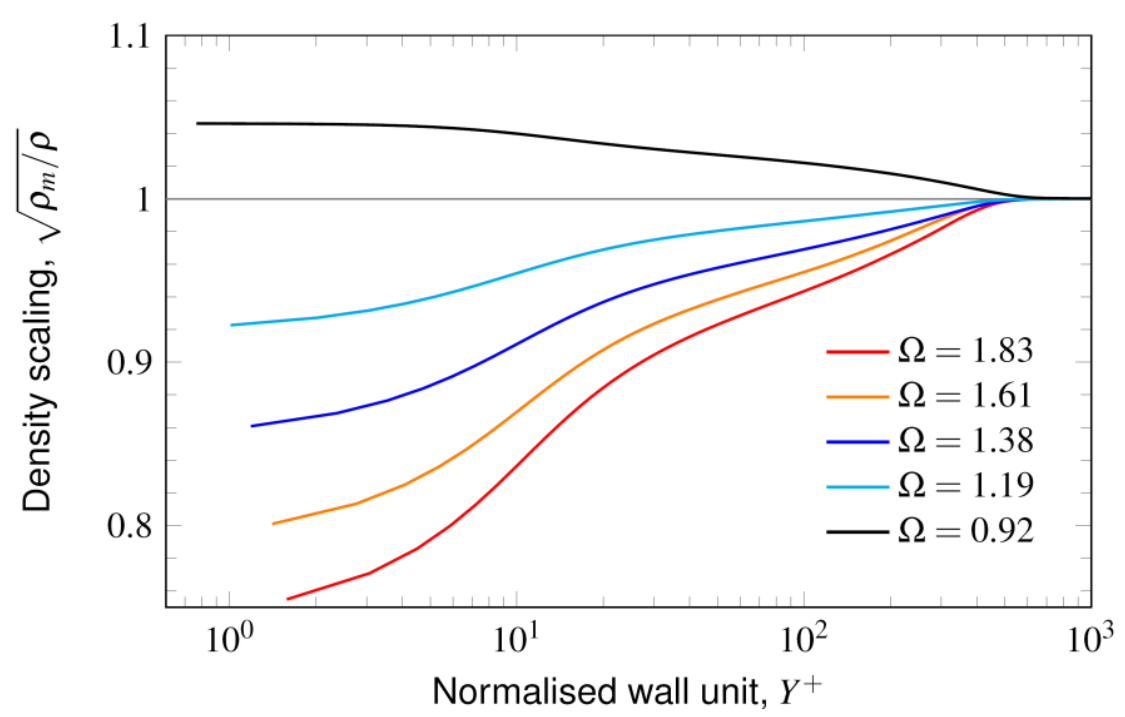

Figure 7.7 - DNS profiles of the density scaling ratio.

\section{Density ratio}

The density ratio used to scale the normalised dissipation profiles is shown in Figure 7.7. This scaling acts throughout the boundary layer but is particularly great close to the wall. For a cooled boundary layer, the change in density acts to reduce the normalised dissipation profiles.

\section{Length-scale}

The relative change in length-scale of the boundary layer can be found by considering transforming the integration length from physical to normalised units as shown in Equation 7.8 .

$$
d y=\underbrace{\frac{\left(\frac{d Y^{+}}{Y^{+}}+\frac{d l}{l}\right)}{d Y^{+} / Y^{+}}}_{\lambda} l d Y^{+}=\left[1+\frac{1}{\bar{\mu}} \frac{d \bar{\mu}}{d Y^{+}} Y^{+}-\frac{1}{2} \frac{1}{\rho} \frac{d \rho}{d Y^{+}} Y^{+}\right] l d Y^{+}
$$

This occurs due to the temperature profile through the boundary layer affecting the density and viscosity profiles. The temperature profile varies both due to compressibility, i.e. high Mach number, and due to heat transfer, i.e. high mainstream to wall temperature ratio, $\Omega$. Figure 7.8 illustrates the relative change in length-scale through the boundary layer. There appears to be two peaks, one around a $Y^{+}$of 10 and another around a $Y^{+}$of 300 . The first peak 


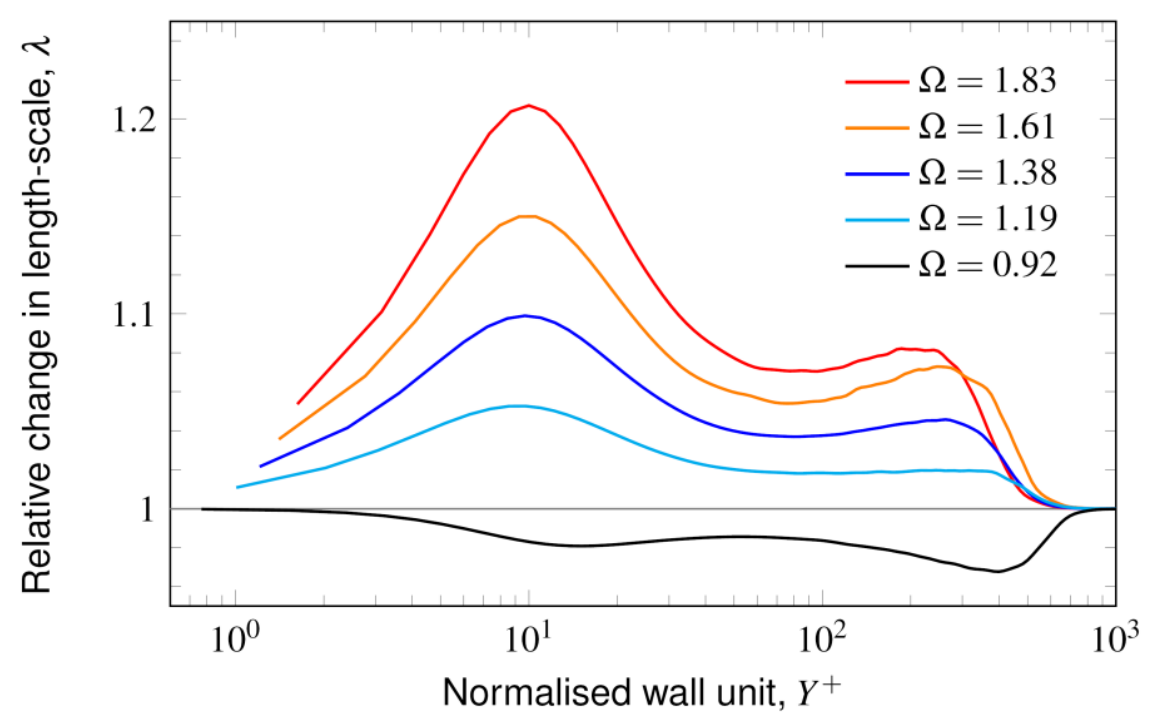

Figure 7.8 - DNS profiles of the relative change in length-scale.

is close to the peak of turbulent dissipation while the second is near the edge of the boundary layer. The effect of compressibility can be seen to reduce the change in length-scale for the adiabatic case (where $\Omega=0.92$ ). The effect of cooling the wall acts to increase the lengthscale.

\section{Combined scaling}

The overall scaling can be obtained by multiplying the effect due to the change in density and length-scale. This is shown in Figure 7.9.

Close to the wall, the change in density dominates as there is only a small change in length-scale. In this region, cooling acts to reduce to mean dissipation profile. Slightly away from the wall, the change in density and length-scale act to cancel each other. This means that cooling has little effect on the production of TKE which is particularly great in this region. Towards the freestream, the change in length-scale dominates. However, in this region the dissipation rates are small, so the effect of this scaling is minimal.

This agrees with intuition that heat transfer has the greatest effect on the mean dissipation profile as they both occur close to wall. However, it appears to contradict the overall result found in Figure 7.4. This will be resolved in the next section. 


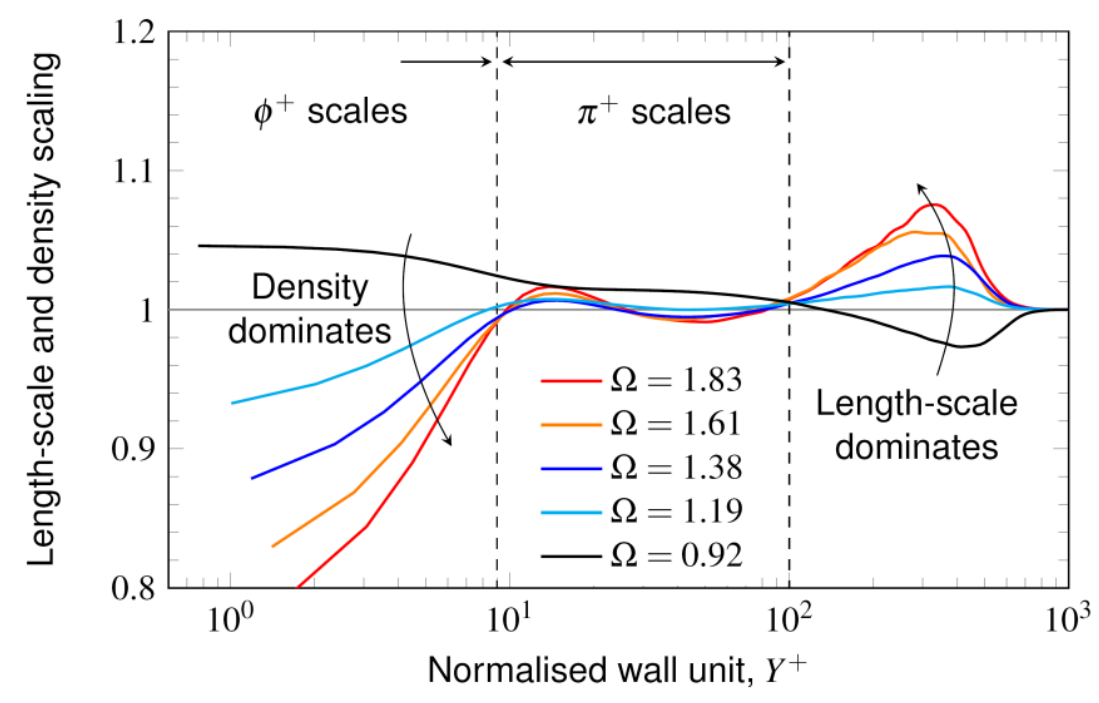

Figure 7.9 - DNS profiles of the overall scaling effect due to heat transfer.

\subsubsection{Relating the change in profile to dissipation coefficient}

The dissipation coefficient expressed on inner units, Equation 7.6, is a product of the skinfriction coefficient and the integrated dissipation profile. The change in the integrated profile of normalised dissipation would suggest that wall cooling would reduce the dissipation coefficient. However, this is not observed because the change in the dissipation profile is balanced by an equivalent change in skin friction coefficient. This can be observed by substituting Equation 7.6 into Equation 7.3 and differentiating:

$$
d\left[\left(\frac{c_{f}}{2}\right)^{\frac{1}{2}}\left(\Phi^{+}+\Pi^{+}\right)\right]=d\left[\frac{H_{32}}{2}\right] \approx 0
$$

Equation 7.9 indicates that any change in the integrated inner unit dissipation profile must be matched by a corresponding change in skin friction as the change in energy shape factor is approximately zero. As the combined density and length-scale change predominantly affects the scales close to the wall, the change in skin friction is due to the change in the integrated mean dissipation profile leading to Equation 7.10. 


$$
d\left[\left(\frac{c_{f}}{2}\right)^{\frac{1}{2}}\right] \approx-\left(\frac{c_{f}}{2}\right)^{\frac{1}{2}} \frac{d \Phi^{+}}{\Phi^{+}+\Pi^{+}}
$$

The mean dissipation and production of TKE contributions can be split into their contributions to the dissipation coefficient, as demonstrated in Equation 7.11:

$$
\Phi=\left(\frac{c_{f}}{2}\right)^{\frac{3}{2}} \Phi^{+}, \quad \Pi=\left(\frac{c_{f}}{2}\right)^{\frac{3}{2}} \Pi^{+}
$$

At engine representative Reynolds numbers, the magnitude of the contribution from the mean dissipation and production of TKE are approximately equal. Using this assumption, Equations 7.10 and 7.11 can be combined to determine the relative change dissipation coefficient.

$$
\begin{aligned}
& \frac{d \Phi}{\Phi} \approx \frac{1}{\Phi} d\left[\left(\frac{c_{f}}{2}\right)^{\frac{3}{2}} \Phi^{+}\right]=-\frac{1}{2} \frac{d \Phi^{+}}{\Phi^{+}} \\
& \frac{d \Pi}{\Pi} \approx \frac{1}{\Pi} d\left[\left(\frac{c_{f}}{2}\right)^{\frac{3}{2}} \Pi^{+}\right] \approx-\frac{3}{2} \frac{d \Phi^{+}}{\Phi^{+}}
\end{aligned}
$$

Equation 7.12 solves the apparent contradiction as to why heat transfer more strongly affects the dissipation coefficient contribution corresponding to the production of TKE than it does the mean dissipation. Heat transfer predominantly scales the profile of mean dissipation which alters the balance with skin friction. The overall effect of this balance acts to partially cancel for the mean component of dissipation coefficient, whereas it does not for the production of TKE. As cooling acts to reduce the mean dissipation profile, this has the opposite effect on skin friction. This results in a rise in the contribution to dissipation coefficient from both mean dissipation and the production of TKE.

Equation 7.12 also shows the interesting result that the change in dissipation coefficient due to production of TKE is three times the change due to mean dissipation (Equation 7.13). 


$$
\frac{d \Pi}{\Pi} \approx 3 \frac{d \Phi}{\Phi}
$$

The ratio of these relative changes is shown for varying wall temperature in Figure 7.10. The DNS and most of the RANS turbulence models predict this ratio to be between 2 and 3 . Only the mixing-length model and $k-\epsilon$ model from Launder \& Spalding (1974) have not captured this coupling. This reduction of this ratio from 3 is due to wall cooling also scaling the production of TKE at values of $Y^{+}$less than 10. The exact value of this ratio will change with $\mathrm{R}_{\delta_{2}}$ and pressure-gradients.

The same result presented here may hold in reverse for adiabatic flows with high mainstream turbulence intensity. The turbulence intensity predominantly will act to rescale the length-scales slightly away from the wall and will be damped in the near-wall region. This means that the production of TKE profile will change while the mean dissipation profile will remain substantially unchanged. Therefore, it would be expected that the change due to mean dissipation is three times the change due to the production of TKE (Equation 7.14).

$$
\frac{d \Phi}{\Phi} \approx 3 \frac{d \Pi}{\Pi}
$$

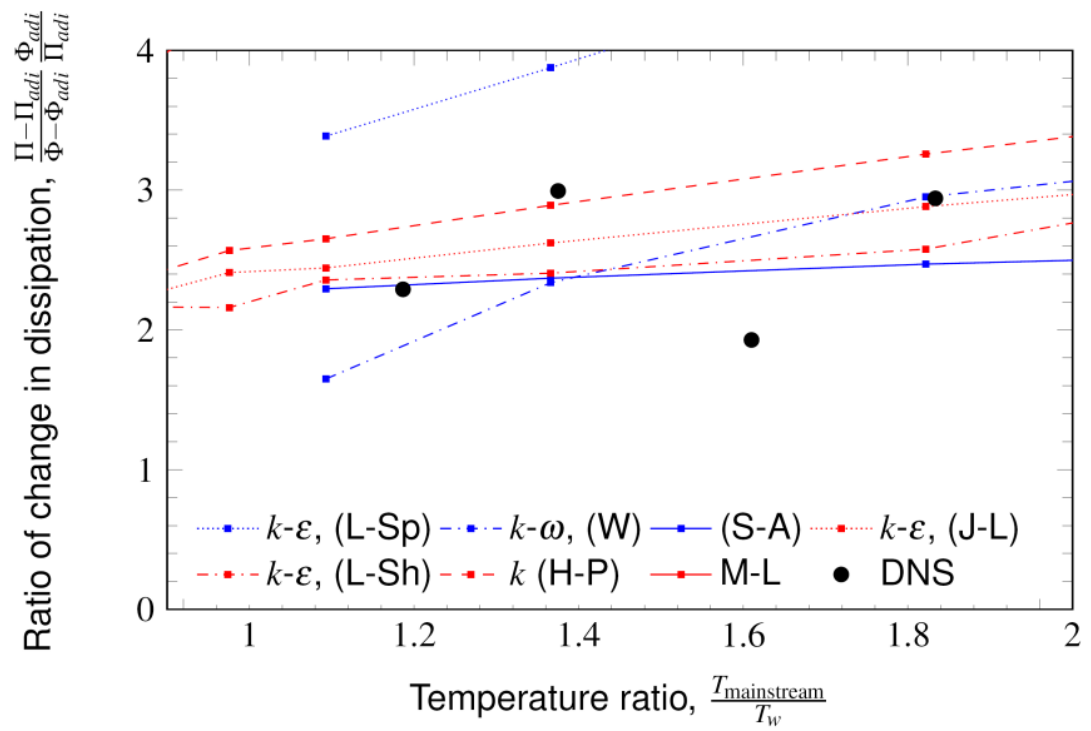

Figure 7.10 - The ratio of the relative change in the effect of the production turbulent kinetic energy to the relative change in the effect of mean dissipation at different mainstream-to-wall temperature ratios. 
This effect can be seen when comparing the low turbulence intensity and high turbulence intensity flat plate dissipation coefficients presented by Folk, Miller, \& Coull (2019). They found the increase in turbulence intensity produced a relative change of $35 \%$ in the mean component and produced a relative change of only $11 \%$ in the fluctuating component. The ratio of the two relative changes is 3.25 .

\subsubsection{Summary}

The effect of heat transfer on the dissipation coefficient is linked to changes in the skin friction coefficient. This was observed using the integral boundary layer methods which relates the ratio of the dissipation and skin friction coefficients to the energy shape factor.

These integral boundary layer methods have been obtained by considering the outer scales, but the dissipation coefficient can also be determined using inner scales. Comparing the two, the change in skin friction coefficient balances the change in the integrated dissipation profile. The effect of heat transfer on this profile can be decomposed into three effects; the nondimensional profiles, the change in density and the change of length-scale. The overall scaling effect of the change in density and length-scale acts to rescale the profile close to the wall, which predominantly affects the mean dissipation profile.

When examining the mean component of dissipation coefficient, the effects due to the change in dissipation profile and change in skin friction coefficient partially cancel. When examining the fluctuation component of dissipation coefficient, only the change in skin friction acts causing a much greater change.

This knowledge could be used to predict the fluctuating component, which is difficult to measure, from mean component, which is easier. For heat transfer, this may have limited accuracy as the change in the fluctuating component is greater than the change in the mean component. For highly turbulent flows, the extrapolation would be better bounded as it is expected that the change in mean component is greater. 


\subsection{Linking dissipation coefficient to pressure-gradient coupling}

In a cooled turbine, significant heat transfer and pressure gradients exist. The integral boundary layer method suggested that the heat transfer also affects dissipation coefficient through a pressure-gradient coupling. This section aims to identify how well this coupling is currently modelled in design. It is divided into two parts which consider laminar and turbulent flow respectively.

\subsubsection{Laminar boundary layer}

For laminar flow, the compressible Blasius solution was numerically solved for cases with both a pressure gradient and heat transfer. Although the velocity profile no longer able to be solved analytically, this does allow more representative flows to be examined.

Figure 7.11 shows the effect of pressure gradient and mainstream-to-wall temperature ratio on laminar flow. The pressure gradient parameter used is $\beta$ from the Blasius equations and the velocity profiles were examined at $R_{\delta_{2}}=400$. The results presented in Figure 7.1 are the values taken along a line of zero pressure gradient. The top two graphs of Figure 7.11 show the dissipation coefficient (left) and the skin friction coefficient (right). The contour distributions broadly agree, but there are substantial differences particularly for adverse pressure gradients. As the contour lines are not straight, this indicates that there is significant coupling between the temperature field and pressure gradient.
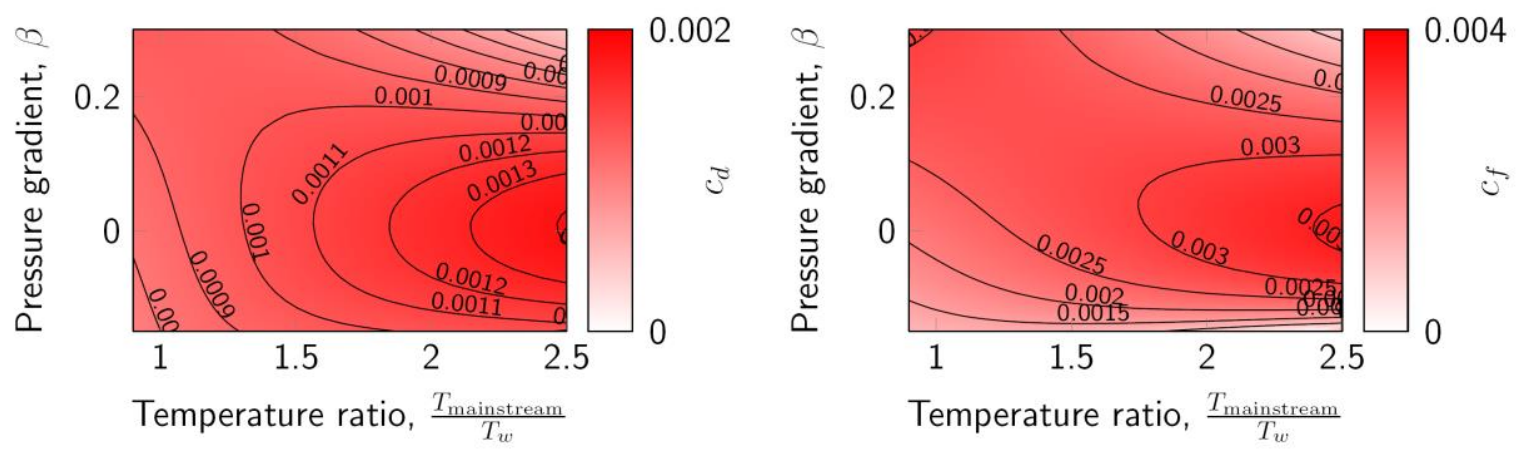

Figure 7.11 - The effect of pressure gradient and mainstream-to-wall temperature ratio on laminar boundary layer flow at $R_{\delta_{2}}=400$. Left, dissipation coefficient. Right, skin friction coefficient. 
To summarise this section. The effect of either heat transfer or pressure gradient can be captured using a simple change of scale. Capture their combined effect requires improved modelling.

\subsubsection{Turbulent boundary layer}

For turbulent flow, RANS turbulence models in TEXSTAN have been used to simulate the effect of both with pressure gradients and heat transfers. This section does not have the benefit of DNS data to provide grounding.

Figure 7.12 shows the effect of pressure gradient and mainstream-to-wall temperature ratio on turbulent dissipation coefficient, modelled by 4 different turbulence models. The pressure gradient parameter used is $\beta$ the Rotta-Clauser parameter as this is more appropriate for turbulent flows and the velocity profiles were examined at $R_{\delta_{2}}=1000$. The results presented in Figure 7.2 are the values taken along a line of zero pressure gradient.

The top two graphs in Figure 7.12 are for the mixing-length model and the one equation model of Hassid \& Poreh (1975). These both predict similar values and behaviour for the dissipation coefficient and suggest that, across the range simulated, heat transfer has a greater relative effect than pressure gradient. The similarity of the two predictions is not too surprising as they both apply a mixing-length in their model.

The bottom two graphs in Figure 7.12 are for the two equation models of Launder \& Sharma (1974) and Jones \& Launder (1973). These both predict similar values and behaviour, but a marked contrast to the previous models. Not only does cooling have a greater predicted effect, as shown earlier in Figure 7.2, but the predicted effect of a pressure gradient is reversed.

For all the graphs, the contour lines are almost straight and evenly spaced. This indicates that the effect of heat transfer is decoupled from the pressure-gradient effect.

There are two possible conclusions to these results. Either that the effect of pressuregradient and the effect of heat transfer are decoupled. This seems surprising due to the evident coupling displayed by the laminar boundary layer. Alternatively, the RANS turbulence models 

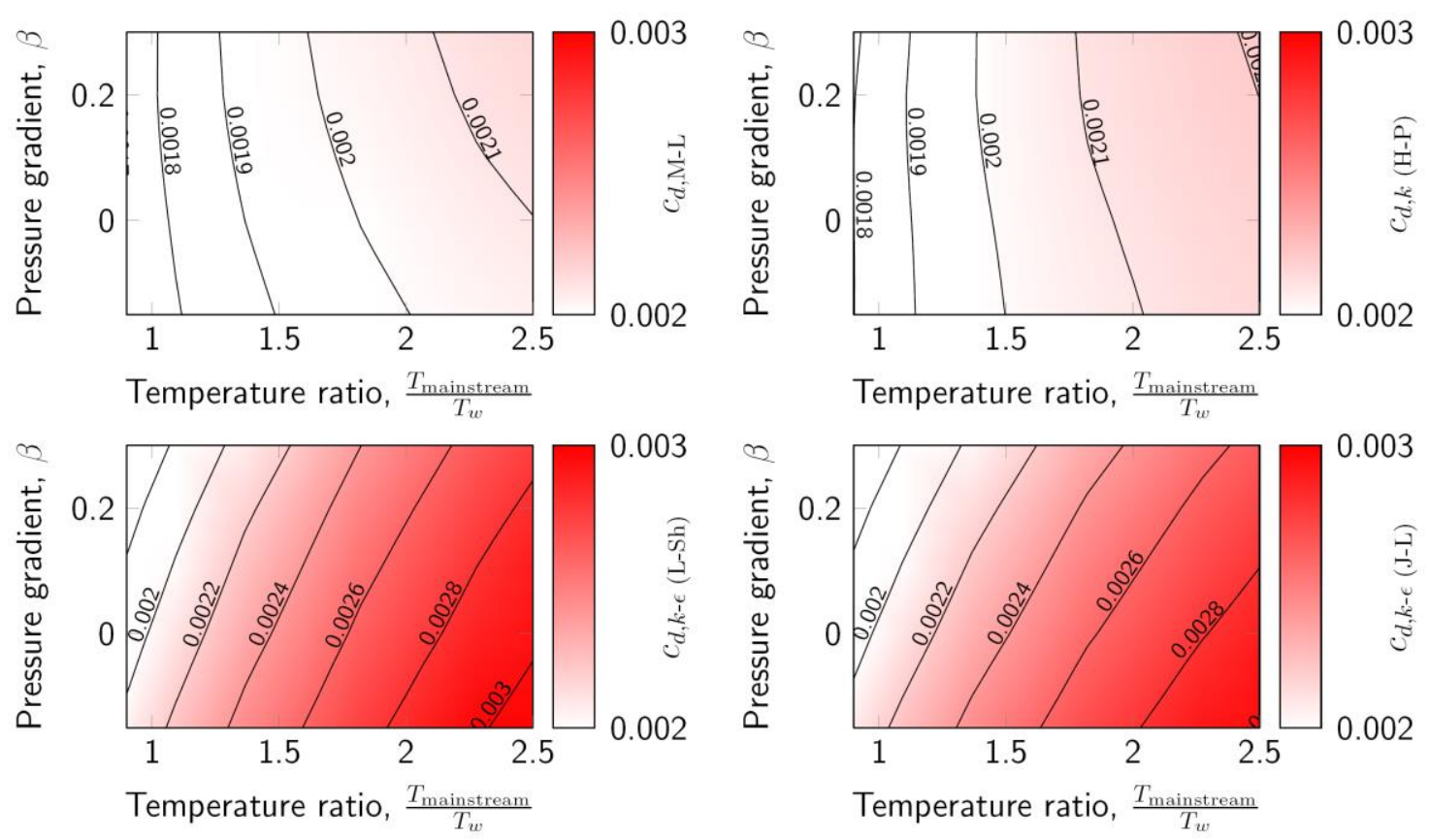

Figure 7.12 - The effect of pressure gradient and mainstream-to-wall temperature ratio on turbulent boundary layer dissipation coefficient at $\mathrm{R}_{\delta_{2}}=1000$ predicted using different turbulence models. Top left, mixinglength. Top right, Hassid \& Poreh. Bottom left, Launder \& Sharma. Bottom right, Jones \& Launder.

are modelling these two effects as if they are decoupled. Future investigations could generate DNS data to improve the accuracy of RANS models to predict the dissipation coefficient.

\subsubsection{Summary}

The laminar flow illustrates that the effect of pressure gradient and heat transfer on the dissipation coefficient are coupled. The opposite outcome is predicted by the RANS models for turbulent flow. The most likely conclusion is that the RANS models are modelling these two effects in a decoupled manner. This could be limiting the accuracy of these model to capture the performance of a cooled turbine.

\subsection{Impact on turbomachinery}

This section examines the loss coefficient of the internally-cooled turbine cascade blade, previously examined in Chapter 5. The results have been obtained using different turbulence 
models in ANSYS Fluent. The previous sections demonstrated that the predicted effect of heat transfer on dissipation coefficient varies between RANS models, with the Spalart-Allmaras model achieving the best agreement with the DNS flat plate results. The RANS models also predicted that the pressure-gradient and heat transfer effects are decoupled. It is unclear which turbulence model is the most accurate in this case.

For a cooled turbine, Figure 7.13 illustrates that, as the mainstream-to-coolant temperature ratio is increased, the predicted loss coefficient rises for all the turbulence models. However, they do not predict the same effect. The Spalart-Allmaras model predicts a $4.3 \%$ rise in loss coefficient while the $k-\omega$ model predicts a rise of just $0.5 \%$. At a temperature ratio of 2 , the difference between the $k-\epsilon$ loss coefficient and $k-\omega$ loss coefficient is $16.5 \%$ while the difference between the S-A model and $k-\omega$ is $10.1 \%$. This highlights the uncertainty in predicting the loss coefficient of a cooled turbine using current RANS methods.

The variation in loss coefficient between the different turbulence models is comparable to the variation between different turbine designs. This means that the optimal choice of turbulence model for cooled turbines is unclear and could lead to different design decisions.

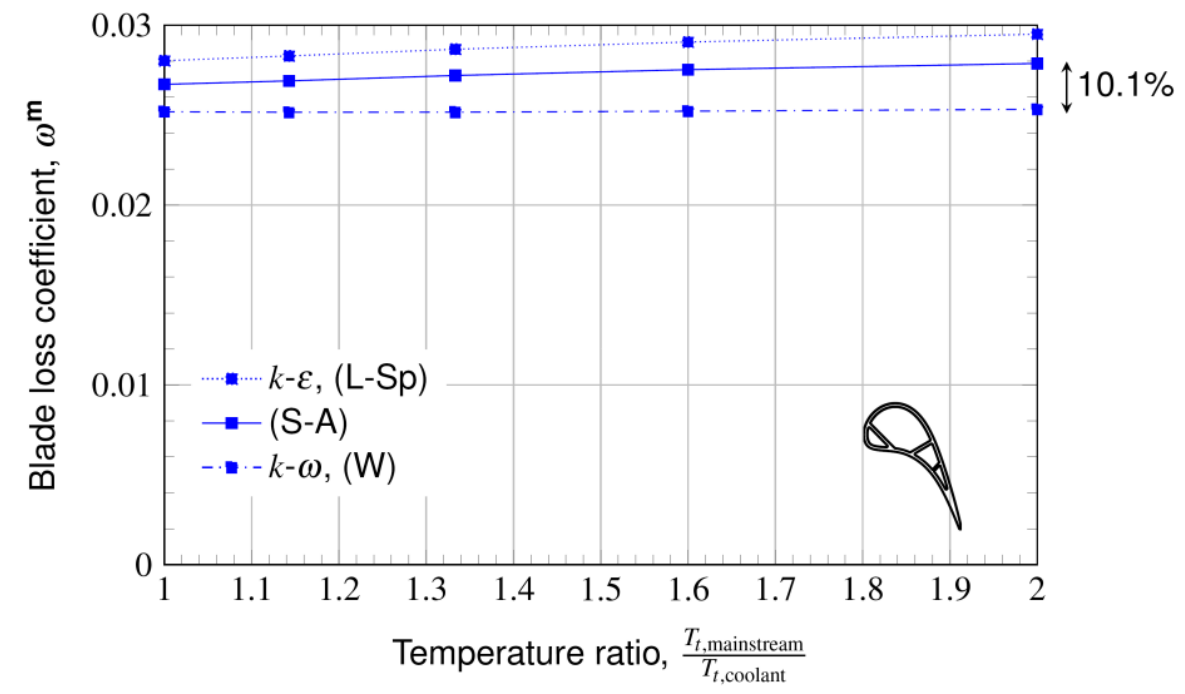

Figure 7.13 - The change in blade loss coefficient with mainstream-to-coolant total temperature ratio using different turbulence models in ANSYS Fluent. 


\subsection{Summary}

This chapter investigated the effect of heat transfer on the boundary layer dissipation coefficient. It was shown that heat transfer acted to change the dissipation coefficient in two ways: through a change in skin friction and by modifying the influence of pressure-gradients.

Examining the flow over a flat plate, it found that the effect of heat transfer on the dissipation coefficient was proportional to how heat transfer affects the skin friction coefficient, for both laminar and turbulent boundary layers. For a laminar flow, the effect of heat transfer is to rescale the boundary layer through thermofluid properties. For turbulent flow, the dissipation coefficient is a balance between skin friction and the integrated dissipation profile. The effect of heat transfer acts to predominantly rescale the integrated dissipation profile. For the mean contribution to dissipation coefficient, the change in mean dissipation profile partially cancels the change in skin-friction coefficient resulting in only a small overall change. For the fluctuating contribution to dissipation coefficient, no such cancellation occurs resulting in a much greater increase. For heat transfer cases, the ratio of the change of fluctuating component to the change of mean component was found to be between 2 and 3. For cases with high turbulence intensity, this ratio is expected to be $1 / 3$.

The effect of a freestream pressure gradient on laminar boundary layers was found to be coupled to the effect of heat transfer. However, for turbulent flow, RANS models predicted that the effect of heat transfer and pressure gradient on the dissipation coefficient were decoupled. This is likely to be a limitation of the RANS methods applied.

For cooled turbines, different RANS models predict different trends in performance. Between a mainstream-to-coolant temperature ratio of 1 and 2 , the $k$ - $\omega$ model predicted a rise in loss coefficient of $0.5 \%$ while the Spalart-Allmaras model predicted a rise of $4.3 \%$. It is unclear which the more accurate model is, and the difference in loss coefficient prediction may lead a designer to choose a sub-optimal blade design. 



\section{Chapter 8 Impact on turbine blade design}

The design of a practical turbine blade is a balance between aerothermal performance and constraints arising from mechanical, structural or material requirements. The new understanding into how heat transfer affects turbine performance changes this balance. The aim of this chapter is to illustrate how the new understanding could improve future blade design. Three practical questions are considered:

\section{What is the aerothermal limit of thermal creation in a turbine cooling system?}

The effect of thermal creation in a turbine blade is driven by forced convection. In the forced convection regime, the same mechanism is responsible for transferring heat and momentum. This means, the same mechanism is responsible for the changes in performance due to thermal creation and viscous dissipation. In this section, the balance between the effect of thermal creation and viscous dissipation is examined for heat transfer between a mainstream and coolant flow.

\section{What type of turbine cooling system maximises thermal creation?}

In Chapter 5 and Chapter 6, thermal creation was shown to improve the loss coefficient of an internally-cooled and film-cooled blade. This section examines what type of turbine blade cooling system would maximise the effect of thermal creation.

\section{Can turbine loss coefficients be better translated from laboratory to engine?}

High-pressure turbine loss coefficients are currently evaluated in a laboratory environment and experience significantly lower temperatures and rates of heat transfer than in the engine. The effect of heat transfer is currently not accounted for in the translation from this laboratory setting into the engine. This section outlines how an improved translation may be achieved. 


\subsection{What is the aerothermal limit of thermal creation in a turbine cooling system?}

In a turbine, heat transfer from the flow to the wall occurs in a regime known as forced convection. In this regime, the mechanism that transfers heat is also responsible for transferring momentum. Flow processes that rely on the transfer of heat and momentum, such as thermal creation and viscous dissipation, are coupled as a result of this shared mechanism. This section seeks to examine how this aerothermal coupling limits the benefit that could be achieved using thermal creation.

Consider two separate streams, a mainstream and a coolant, with heat transfer between them. Both streams are in the forced convection regime and a small fluid element from each stream will be examined. As heat is transferred, the change in ability to extract work using a turbine from the system will be discussed.

For each stream, the ability to extract work using a turbine is characterised by the flow euergy, $\mathbf{m}_{\mathbf{f}}$. This is presented in Equation 8.1.

$$
\mathbf{m}_{\mathbf{f}}=h\left(1-\left(\frac{p_{0}}{p}\right)^{\frac{\gamma-1}{\gamma}}\right)+\frac{1}{2} V^{2}=h_{t}\left(1-\left(\frac{p_{0}}{p_{t}}\right)^{\frac{\gamma-1}{\gamma}}\right)
$$

The fractional change in flow euergy for a fluid element is shown in Equation 8.2.

$$
\frac{d \mathbf{m}_{\mathbf{f}}}{\mathbf{m}_{\mathbf{f}}}=\frac{d T_{t}}{T_{t}}+\underbrace{\frac{\gamma-1}{\gamma} \frac{\left(\frac{p_{0}}{p_{t}}\right)^{\frac{\gamma-1}{\gamma}}}{1-\left(\frac{p_{0}}{p_{t}}\right)^{\frac{\gamma-1}{\gamma}}} \frac{d p_{t}}{p_{t}}}_{A}
$$

The change in flow euergy can be expressed by the changes in total temperature and total pressure. Applying the method used by Shapiro \& Hawthorne (1947), the change in total pressure can be split into a contribution due to heat transfer and a contribution due to friction. 
This allows the change in flow euergy to be expressed as a result of heat transfer and friction, as shown in Equation 8.3.

$$
\frac{d \mathbf{m}_{\mathbf{f}}}{\mathbf{m}_{\mathbf{f}}}=\underbrace{\left(1-A \frac{\gamma \mathrm{M}^{2}}{2}\right) \frac{d T_{t}}{T_{t}}}_{\text {Due to heat transfer }}-\underbrace{A \frac{\gamma \mathrm{M}^{2}}{2}\left(4 f \frac{d x}{D}\right)}_{\text {Due to friction }}
$$

In the forced convection regime, the aerothermal coupling between heat transfer and friction is known as the Reynolds analogy. Equation 8.4 considers the heat leaving a fluid element and demonstrates that the Reynolds analogy links the change in total temperature to the effect of friction. This means that, for each stream, heat transfer cannot independently change flow euergy as there will always be a contribution due to friction.

$$
d Q=h \frac{d T_{t}}{T_{t}}=h\left(\frac{T_{w}}{T_{t}}-\frac{T_{w a}}{T_{t}}\right) 2 f \frac{d x}{D}
$$

Considering the two streams together, then the heat leaving one will arrive into the other. This heat transfer links the change in one stream to the change in the other. Applying conservation of energy leads to Equation 8.5.

$$
\dot{m}_{m} d Q_{m}=-\dot{m}_{c} d Q_{c}
$$

The total change in the flow euergy for the two streams can be found be considering the change of each stream as shown in Equation 8.6. As the heat leaves one stream and enters the other, this heat transfer across differences in Joule cycle efficiency results in the effect of thermal creation, $\Phi_{t h}^{m}$. Both streams also have losses due to friction resulting in the effect of viscous dissipation, $\Phi_{v}^{m}$. As the effect of heat transfer and friction are coupled, so too are the effect of thermal creation and viscous dissipation.

$$
d \mathbf{M}_{\mathbf{f}}=\dot{m}_{m} \mathbf{m}_{\mathbf{f}, m} \frac{d \mathbf{m}_{\mathbf{f}, m}}{\mathbf{m}_{\mathbf{f}, m}}+\dot{m}_{c} \mathbf{m}_{\mathbf{f}, c} \frac{d \mathbf{m}_{\mathbf{f}, c}}{\mathbf{m}_{\mathbf{f}, c}}
$$




$$
\rightarrow d \mathbf{M}_{\mathbf{f}}=\Phi_{t h}^{\mathbf{m}}-\Phi_{v}^{\mathbf{m}}=\Phi_{v}^{\mathbf{m}}\left(\frac{\Phi_{t h}^{\mathrm{m}}}{\Phi_{v}^{\mathrm{m}}}-1\right)
$$

The ratio of the effect of thermal creation to the effect of viscous dissipation indicates the maximum reduction in loss that could be achieved. If the ratio is greater than unity, then thermal creation could be employed to overcome the effects of viscous dissipation. This would indicate installing additional heat exchangers might be beneficial.

The magnitude of this aerothermal ratio can be determined for the two streams. Details of the derivation have been provided in Appendix D.1. If the total pressure and ratio of specific heat of the two streams are the same, then the aerothermal ratio is expressed as a function of Mach number and temperature alone. This is expressed in Equation 8.7.

$$
\frac{\Phi_{t h}^{\mathrm{m}}}{\Phi_{v}^{\mathrm{m}}}=\frac{-\frac{1}{2}\left(1-\frac{\mathrm{M}_{c}^{2}}{\mathrm{M}_{m}^{2}}\right)\left(\frac{T_{w}}{T_{t}}-\frac{T_{w a}}{T_{t}}\right)_{m}}{1-\frac{\mathrm{M}_{c}^{2}}{\mathrm{M}_{m}^{2}} \frac{\left(\frac{T_{w}}{T_{t}}-\frac{T_{w a}}{T_{t}}\right)_{m}}{\left(\frac{T_{w}}{T_{t}}-\frac{T_{w a}}{T_{t}}\right)_{c}}}
$$

For a cooled turbine blade, the maximum ratio of the effect of thermal creation to the effect of viscous dissipation can be estimated using the two streams. The operating conditions presented by Young \& Wilcock (2002b) have been applied.
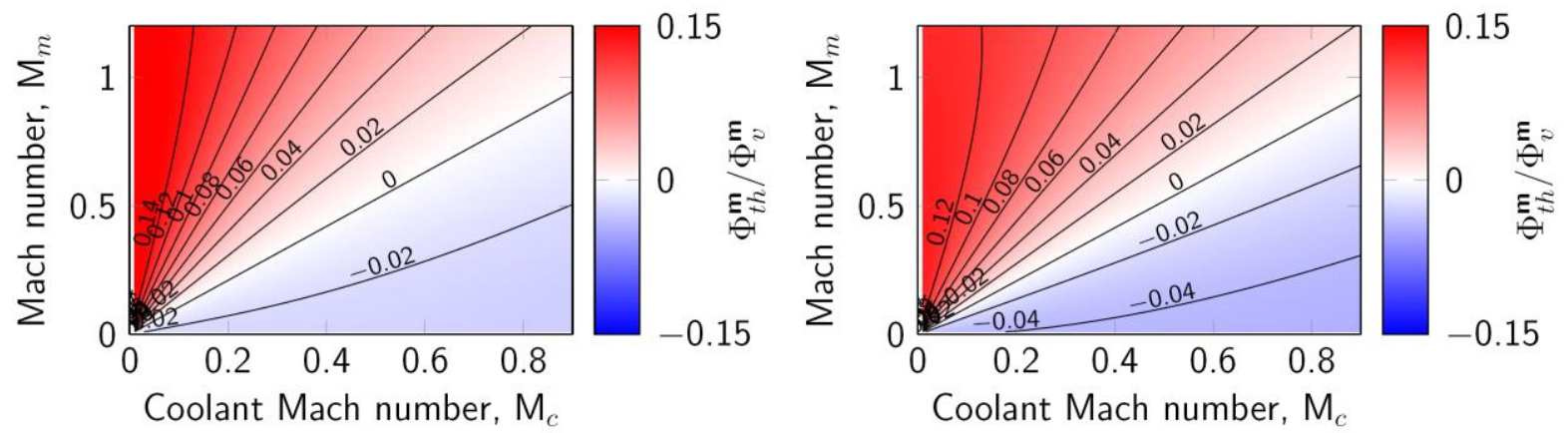

Figure 8.1 - Contours showing the ratio of the effect of thermal creation to the effect of viscous dissipation as the Mach number of the mainstream and coolant are varied. The values are for the turbine stator blade (left) and the turbine rotor blade (right) presented by Young \& Wilcock (2002b). 
Figure 8.1 illustrates the aerothermal ratio for a turbine stator (left) and rotor (right). As the difference between the mainstream and coolant Mach number increases, the magnitude of the effect of thermal creation also increases. At engine conditions, the aerothermal ratio for both the stator and rotor is approximately $8 \%$. The engine Mach numbers also suggests that a greater relative improvement in performance could be obtained by decreasing the coolant Mach number rather than increasing the mainstream Mach number.

This section has shown that the improvement in turbine performance resulting from thermal creation is coupled to the detriment in turbine performance resulting from viscous dissipation. For a turbine cooling system, this means that thermal creation will result in some amount of viscous dissipation. At current engine conditions, thermal recuperation could reduce the blade loss coefficient by approximately $8 \%$.

\subsection{What type of turbine cooling system maximises thermal creation?}

Thermal creation has shown that where heat is transferred into the cooling system can alter the component efficiency. This section seeks to investigate what type of cooling system would maximise this effect.

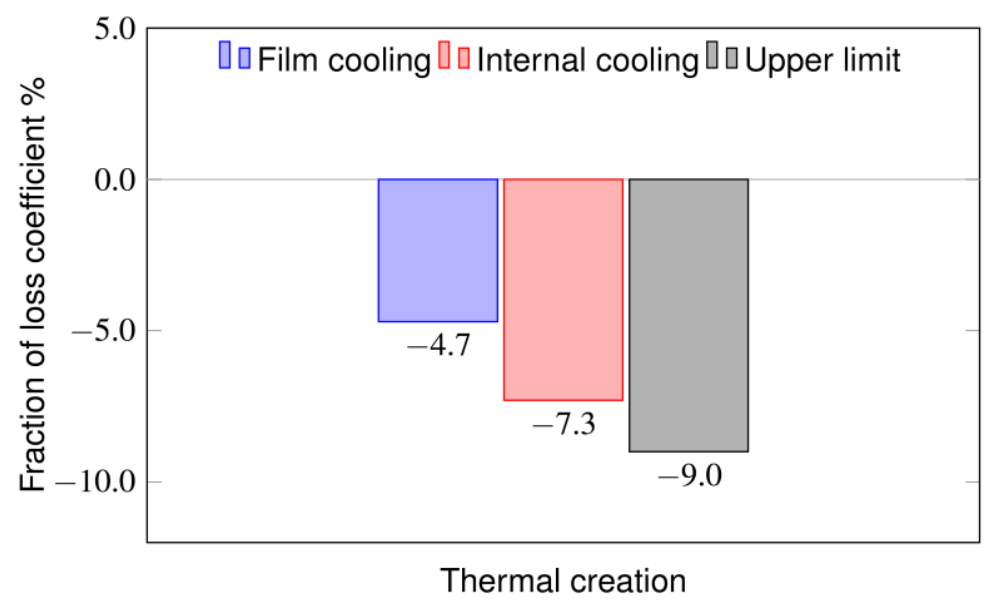

Figure 8.2 - Fractional change in loss coefficient due to thermal creation for film-cooling (blue), internalcooling (red) and the internal-cooling upper limit (grey). 
Three cases are considered that share the blade profile but employ different cooling systems. Each case considers the impact of different cooling strategies on the loss coefficient of the blade. The change in loss coefficient due to thermal creation, relative to an uncooled turbine blade, is presented in Figure 8.2.

The blue bar in Figure 8.2 shows the impact of adding the external film cooling system, described in Chapter 6, on the loss coefficient of the blade. This is characteristic of a modern cooling system. The presence of thermal creation due to heat transfer through the wall of the turbine reduces the loss coefficient by $4.7 \%$.

The red bar in Figure 8.2 shows the impact of a future cooling scheme. In this scheme the external film cooling is removed, and all cooling is achieved through internal cooling alone. Such a design may be realised by improved materials and by improved internal cooling effectiveness. Because the heat flux through the blade wall rises, the magnitude of the thermal creation term increases. This offers the exciting prospect of reducing the profile loss coefficient by $7.3 \%$.

Finally, the grey bar in Figure 8.2 shows the effect of tailoring the location of heat transfer into the blade surface. In practice, this would be undesirable because the primary aim of the cooling flow is to cool the whole blade surface. However, it is practical way of estimating the upper theoretical limit on the magnitude of the thermal creation term. This shows the maximum size of the thermal creation term could reduce the profile loss coefficient by $9.0 \%$.

These three cases demonstrate that the maximum effect of thermal creation is achieved when both the heat flux magnitude is increased, and the heat flux location is tailored. The magnitude of thermal-creation is significant even for current cooling systems. Future internally-cooled blade designs may be able increase the effect of thermal creation and to achieve almost double the benefit. 


\subsection{Can turbine loss coefficients be better translated from laboratory to engine?}

In industry, turbine blade loss coefficients are evaluated in a laboratory environment. These environments seek to match the aerodynamic conditions experienced by the blade in the engine. However, the heat transfer rate is not matched. The effect of heat transfer on performance, through either thermal creation or changing the boundary layer dissipation coefficient, are not currently accounted for. This section will demonstrate how accounting for these additional effects using the film-cooled blade that was investigated in Chapter 6.

The relative change in loss coefficient compared to an adiabatic blade with the same external profile is presented in Figure 8.3. The loss has been decomposed into the effect due to the boundary layer, cooling holes, wake and thermal creation using the zonal method presented in Chapter 6.

The blue bars indicate the change in the loss coefficient by adding external filmcooling. The greatest contribution to the change is due to the cooling holes, which is currently accounted for in industrial design. However, the effect of heat transfer on the boundary layer dissipation coefficient and the subsequent effect on wake mixing are not accurately accounted.

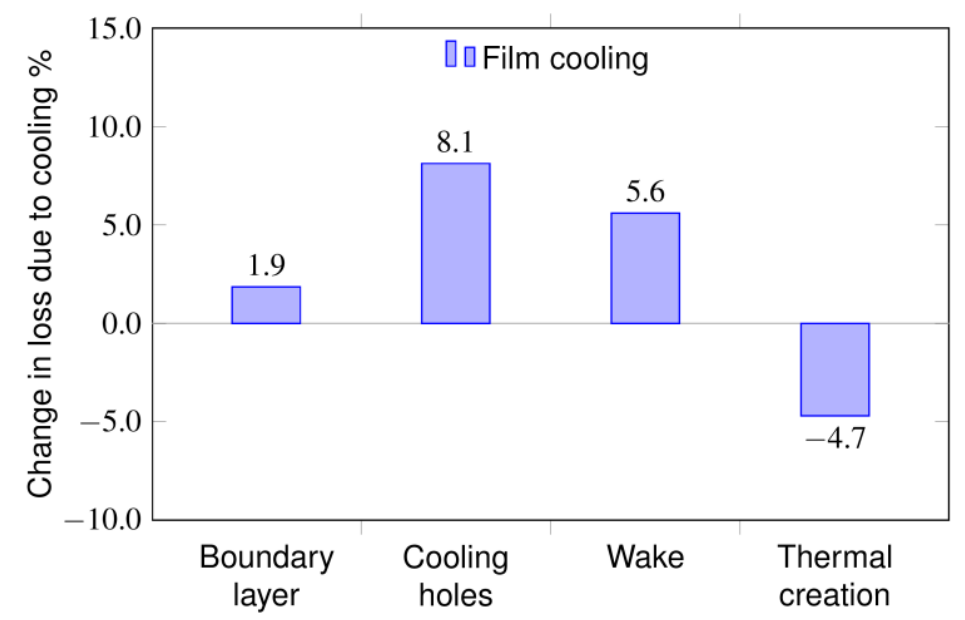

Figure 8.3 - The change in loss coefficient between a film-cooled and adiabatic blade due to the boundary layer, cooling holes, wake and thermal creation. 
This is partially because the complex aerothermal processes are difficult to experimentally verify and partially because the effect of thermal creation, which is also not accounted for, acts to cancel their impact. For the film-cooled design, the combined change due to the boundary layers, wake and thermal creation represents $25.7 \%$ of the total change in loss coefficient.

This highlights two points. Firstly, a significant error in the translation between laboratory and engine may occur by not modelling the effect of heat transfer. Secondly, a future turbine blade cooling system could reduce the loss coefficient of a blade row. Using the current method of translation, neither the error nor the potential loss reduction due to heat transfer would be seen.

\subsection{Summary}

This chapter has considered three practical questions:

\section{What is the aerothermal limit of thermal creation in a turbine cooling system?}

The increase in turbine performance resulting from thermal creation is coupled to decrease resulting from viscous dissipation. This is because, for forced convection, the mechanism responsible for the transfer of heat and momentum are analogous. At engine conditions, thermal recuperation could reduce the loss coefficient of a stator or rotor blade by approximately $8 \%$. This could be increased by increasing the ratio of mainstream-to-coolant Mach number.

\section{What type of turbine cooling system maximises thermal creation?}

The greatest value of thermal creation occurs when heat transfer occurs across differences in Joule cycle efficiency. Both the greatest rate of heat transfer across the blade walls and the greatest difference in Joule cycle efficiency can be achieved using an internally-cooled design. The higher rates of heat transfer are required to maintain the same blade metal temperatures without the protective cooling film. The higher difference in Joule cycle efficiency are possible as the pressure of the coolant passage could vary independently of the mainstream. This allows a future cooling system to increase the magnitude of the heat flux, but also tailor its location. 
Where feasible, transferring heat from the mainstream along the suction-side boundary layer into an internally cooled blade would maximise the effect of thermal creation.

3. Can turbine loss coefficients be better translated from laboratory to engine?

Understanding the effect of heat transfer has two benefits when translating blade loss coefficients. The first is that the effect of heat transfer on the blade loss coefficient can be modelled, improving the accuracy of this translation. The second is that a turbine blade cooling system could be designed that can act to reduce the loss coefficient of a blade row. 



\section{Chapter 9 Conclusions and future work}

The problem of how to develop a general method to determine the performance of complex cooled engine components, which has stood for over 60 years, has been solved. Previous studies were limited by the exergy-based methods available, which directly contradict the experience of industrial turbine designers. In this thesis, the contradiction was solved by applying a new method called euergy. This general method, introduced by Miller, represents the true aspiration of the turbomachinery designer (i.e. to design reversible adiabatic devices) and exhibits the behaviour observed by industrial designers. The generality of the method allows cooled components, however complex, to be systematically analysed for the first time. By applying the euergy method, how heat transfer affects performance was examined.

This chapter is divided into two sections, which focus on the major findings of this study and recommendations for future work respectively.

\subsection{Major findings}

The major findings of this thesis are summarised in five sections, which correspond to the results from Chapters $4,5,6,7$, and 8 respectively.

\subsubsection{Importance of value placed on heat}

Comparing the general methods used to account for aerodynamic performance, it was found that the key difference is the value they place on heat relative to work. This value reflects the ability to extract work from heat addition - acting like a local thermal efficiency. In the exergy method, the value of heat is set by the Carnot cycle efficiency. In the euergy method, the value of heat is set by the Joule (Brayton) cycle efficiency. The power-balance method sets the value of heat based upon a linearization of the Joule cycle efficiency. 
The value placed on heat directly affects ideal work as it is used to set the fraction of the internal energy that could be extracted. In the exergy method, the ideal work reflects the performance of a universal reversible machine that has access to all reversible processes. In the euergy method, the ideal work reflects the performance of a reversible adiabatic turbine. The ideal work set by the euergy method reflects the ideal performance of a turbomachine - a reversible adiabatic expansion. Whenever heat is transferred in the flow or across the wall, the euergy method values this heat based upon the Joule cycle efficiency, illustrated in Figure 9.1.

In this thesis, a thermodynamic method has been referred to as general if local flow processes within a control volume are linked to their effect on the control volume surface for any system. However, not all these general methods are equally well-founded. With the exergy and euergy methods, there is a clear process that corresponds with ideal work and consequently clear concepts of availability and irreversibility. The power-balance method demonstrates that some methods are generalizable, but the process that represents ideal work remains unclear.

For turbomachinery flows, the exergy and power-balance methods are approximations that are valid for adiabatic or incompressible flow. Only the euergy method agrees with industrial experience and reflects the aims of a turbine designer, to design highly-efficient turbomachines.

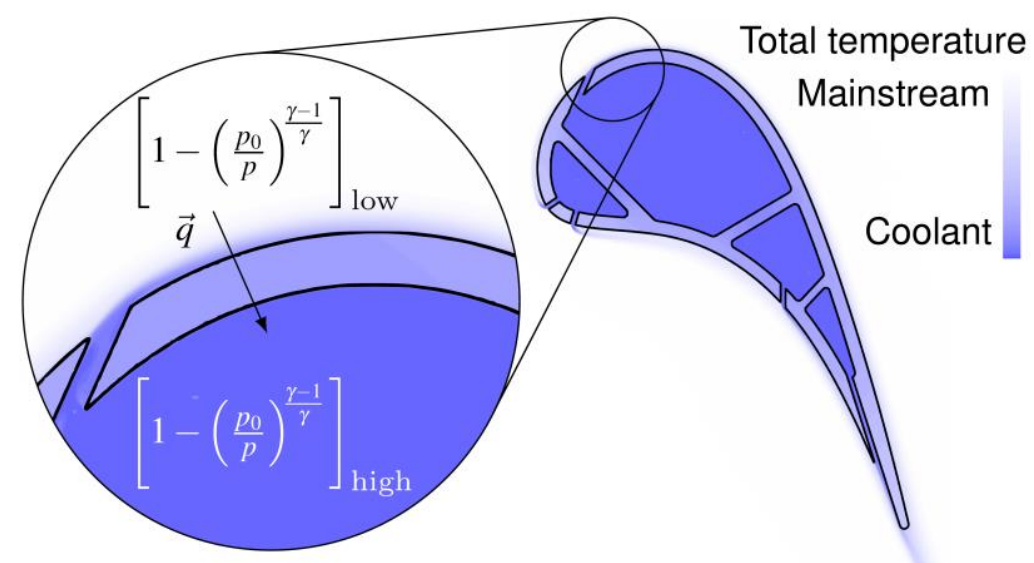

Figure 9.1 - Heat transfer across a difference in Joule cycle efficiency (conjugate CFD solution). 


\subsubsection{Effect of heat transfer on turbine loss mechanisms}

Heat transfer can affect loss mechanisms in three different ways; the effect of heat transfer between streams, the value of viscous reheat and the magnitude of dissipation.

It was shown that the effect of heat transfer between streams can be viewed as a form of thermal recuperation; recovering energy from the exhaust that would otherwise be wasted. This happens when heat is transferred across gradients in local thermal efficiency. For the euergy method, this occurs when heat is transferred across gradients in pressure in a process called thermal creation. This mechanism is not currently accounted for in turbine design and could improve performance.

The reheat effect is due to the rise in internal energy as kinetic energy is dissipated. This rise is equivalent to a local heat addition, which is valued by the exergy method using the Carnot cycle efficiency and by the euergy method using the Joule cycle efficiency. For a cooled turbine, the profile of Carnot cycle efficiency and Joule cycle efficiency through the boundary layer are different, resulting in different values being placed on the increase in internal energy. This means that the same amount of viscous dissipation could affect the exergy and euergy methods differently. Even the viscous component of the exergy method could be misleading to a cooled turbine designer.

The magnitude of dissipation can change due to heat transfer. This can be captured by the effect of heat transfer on dissipation coefficient which is summarised in Section 9.1.4.

\subsubsection{Loss models for cooled turbines}

The effect of heat transfer on turbine performance is not currently accounted for in design. The effect of heat transfer has been distilled into models to capture the effect of thermal creation and viscous dissipation.

First, $90 \%$ of the effect of thermal creation was due to heat transfer across the walls of the turbine blade. This can be accurately modelled by considering the heat flux leaving mainstream through the blade surface. This indicates that accurate performance predictions could be achieved only solving the mainstream flow. 
Second, the effect of viscous dissipation was modelled in the boundary layers, the cooling holes and the trailing edge. It was shown that these regions could be modelled effectively by updating existing techniques. Many of the existing models have been developed to capture the underlying physics using either an exergy-based method or by decomposing an adiabatic loss coefficient. The effect of heat transfer was accurately modelled by updating these models using the euergy method. This reduces the barrier for industrial designers to adopt the euergy method to calculate turbine performance.

\subsubsection{Effect of heat transfer on boundary layer dissipation coefficient}

For a flat plate, both the Blasius solutions and the DNS results validated the framework proposed by the integral boundary layer method that the effect of heat transfer on dissipation coefficient and skin friction coefficient is similar. The effect of heat transfer can be viewed as a balance between the dissipation through the boundary layer, the skin friction at the wall and the shape of the boundary layer. This results in a trade between skin friction at the wall and the profile of shear stresses through the boundary layer. For a laminar flow, the effect of heat transfer is to rescale the boundary layer profile through change in thermofluid properties. For turbulent flow, the effect of heat transfer is also to rescale the boundary layer profile. However, this rescaling predominantly acts close to the wall altering the mean dissipation profile.

Examining the mean contribution to the dissipation coefficient, the effect due to the change in profile is partially cancelled by the associated change in skin friction coefficient. No such cancellation occurs for the contributions from the production of turbulent kinetic energy, resulting in a much greater increase. For heat transfer cases, the ratio of the change of fluctuating component to the change of mean component was found to be between 2 and 3 . For cases with high turbulence intensity, this ratio is expected to be approximately $1 / 3$.

For a turbine blade, different turbulence models predicted different behaviour due to cooling. As the heat transfer rate was increased, the $k-\omega$ model predicted a rise in loss coefficient of $0.5 \%$ while the Spalart-Allmaras model predicted a rise of $4.3 \%$. This difference is significant enough that a sub-optimal blade design may be chosen. 


\subsubsection{Future turbine blade design}

The effect of the newly discovered loss mechanism, thermal creation, allows the turbine cooling system to operate like a recuperator. In practice, heat transfer is coupled to viscous dissipation. Although this restricts thermal creation, it could still reduce the loss coefficient of a stator or rotor blade by approximately $8 \%$ at engine conditions.

In the future, the thermal creation effect could be maximised using an internally-cooled design. Compared to a traditional film-cooled design, higher rates of heat transfer are required to maintain the same blade temperatures. In addition, a greater difference in Joule cycle efficiency could be achieved as the coolant pressure is independent of the mainstream. At current engine conditions, and all the heat transfer occurring across the greatest difference in Joule cycle efficiency, then thermal creation could act to reduce the loss coefficient by $9 \%$.

This new understanding is particularly helpful in translating loss coefficients measured in laboratory settings to their engine value. Currently the effect of heat transfer is not modelled, meaning that the translation for new designs may not be correct. By including this effect, a turbine blade cooling system could be designed that reduces the loss coefficient of a blade row.

\subsection{Recommendations for future work}

In this section, 3 avenues of future work are highlighted.

\subsubsection{New class of general methods}

The euergy method is better suited to examine turbomachinery flows than the exergy method because its perception of ideal work is better aligned with the aspirations of the practical device. However, there are many other practical devices that have a different perception of ideal work.

A new class of general method could be developed to address the discrepancy experienced by other industries between the exergy method and their practical process. The development of a new class of general methods would be very powerful and facilitate a wide range of improved practical thermodynamic devices. 


\subsubsection{Linking heat transfer with thermodynamics}

Historically, the fields of heat transfer and thermodynamics have been distinct. Heat transfer has traditionally focussed on the rate at which the heat is moved, while thermodynamics has focussed on the relationship between heat and work.

However, in a practical device both fields are important and intertwined. A general method can link these two fields. This has been demonstrated in this thesis by applying the euergy method to examine the effect of heat transfer on the thermodynamic performance. Using this approach, the effect of heat transfer on many practical processes could be examined.

An example is to enhance waste heat recovery. All industrial and power generation processes produce heat which is often released into the environment. This low-grade heat has a much lower temperature than a typical gas turbine. At these lower temperatures, air is no longer a good fluid to drive a turbine. A greater efficiency is achieved using fluids that are well suited to this temperature range, such as those found in domestic refrigerators. However, an even greater efficiency may be possible with a new fluid. As well as geometry, the euergy method could indicated how the fluid itself should be optimised.

\subsubsection{Cycle analysis}

This thesis has focussed on the effect of heat transfer on loss mechanisms occurring within the component. However, there are also many cycle level changes that can affect efficiency.

On example is the transition from a simple gas turbine cycle to a combined cycle. This is particularly useful as many new developments have a gas turbine and steam turbine. The euergy method may be able to link across the two, but this is currently unclear.

Another example is how to build in cycle information into component analysis. This is crucial when accounting for the addition of cooling air - whether to charge it to that stage or not. This also has implication for the cooling air as the efficiency of the secondary air system is less than the efficiency of the mainstream, despite both streams having the same ideal work. 


\section{References}

Ainley, D. G., and C. R. Mathieson. 1951. "A Method of Performance Estimation for AxialFlow Turbines." Aeronautical Research Council Reports And Memoranda, N. 2974 2974 (2974).

Anderson, John D. 1989. Hypersonic and High-Temperature Gas Dynamics. AIAA Education Series. https://doi.org/10.2514/4.105142.

Anderson, Mark, Rahul Patwa, and Yung C. Shin. 2006. "Laser-Assisted Machining of Inconel 718 with an Economic Analysis." International Journal of Machine Tools and Manufacture 46 (14): 1879-91. https://doi.org/10.1016/j.ijmachtools.2005.11.005.

Atkins, Nicholas R., and Roger W. Ainsworth. 2012. "Turbine Aerodynamic Performance Measurement Under Nonadiabatic Conditions." Journal of Turbomachinery 134 (6). https://doi.org/10.1115/1.4004857.

Bejan, Adrian. 1979. “A Study Of Entropy Generation In Fundamental Convective Heat Transfer." ASME Journal of Heat Transfer 101: 718-25.

Blackburn, Robert J., and Robert J. Miller. 2017. "The Rayleigh Efficiency of Pressure Gain Combustors.” Journal of Propulsion and Power 33 (1): 51-61. https://doi.org/10.2514/1.B36073.

Bons, J. 2005. "A Critical Assessment of Reynolds Analogy for Turbine Flows." Journal of Heat Transfer 127: 472-85. https://doi.org/10.1115/1.1861919.

Brown, L. E. 1972. "Axial Flow Compressor and Turbine Loss Coefficients: A Comparison of Several Parameters.” ASME Conference Proceedings, no. 72-GT-18.

Casey, Michael V. 2007. "Accounting for Losses and Definitions of Efficiency in Turbomachinery Stages." Proceedings of the Institution of Mechanical Engineers, Part A: Journal of Power and Energy 221 (6): 735-43. https://doi.org/10.1243/09576509JPE459.

Craig, H. R. M.; Cox, H. J. A. 1971. "Performance Estimation of Axial Flow Turbines." Proceedings of Institute of Mechanical Engineers1 185.

Crawford, Michael E. 1985. "TEXSTAN Program.” Univ. of Texas at Austin.

Crawford, Michael E., and W. M. Kays. 1976. "STAN5 - A Program For Numerical Computation of Two-Dimensional Internal and External Boundary Layer Flows." Stanford, California.

Denton, J. D. 1993. "Loss Mechanisms in Turbomachines." In Transactions of the ASME. https://doi.org/10.1115/1.2929299.

Denton, J D, and L Xu. 2018. "The Trailing Edge Loss of Transonic Turbine Blades." 
Drela, Mark. 2009. "Power Balance in Aerodynamic Flows." AIAA Journal 47 (7): 1761-71. https://doi.org/10.2514/1.42409.

Drela, Mark, and Michael B Gilest. 1986. "Viscous-Inviscid Analysis of Transonic and Low Reynolds Number Airfoils" 25 (10).

Dunham, J.; Came, P. M. 1970. "Improvements to the Ainley-Mathieson Method of Turbine Performance Prediction." ASME Journal of Engineering for Gas Turbines and Power 92(3): 252-56. https://doi.org/10.1115/1.3445349.

Folk, Masha, Robert J. Miller, and John D. Coull. 2019. "The Impact of Combustor Turbulence on Turbine Loss Mechanisms,” 1-15. https://doi.org/10.1115/gt2019-90307.

Goyne, C. P., R. J. Stalker, and A. Paull. 2003. "Skin-Friction Measurements in HighEnthalpy Hypersonic Boundary Layers." Journal of Fluid Mechanics 485: 1-32. https://doi.org/10.1017/S0022112003003975.

Greitzer, E. M., C. S. Tan, and M. B. Graf. 2004. Internal Flows. Cambridge University Press. https://doi.org/https://doi.org/10.1017/CBO9780511616709.

Hartsel, J. E. 1972. "Prediction of Effects of Mass-Transfer Cooling on the Blade-Row Efficiency of Turbine Airfoils." AIAA 10th Aerospace Sciences Meeting.

Hassid, S., and M. Poreh. 1975. "A Turbulent Energy Model for Flows with Drag Reduction.," no. 5: 234-41.

Hawthorne, W. R. 1956. "The Thermodynamics of Cooled Turbines, Parts I and II.” In Proceedings of ASME Turbo Expo, 78:1765-81.

Herwig, H., and F. Kock. 2006. "Local Entropy Production in Turbulent Shear Flows: A Tool for Evaluating Heat Transfer Performance." Journal of Thermal Science 15 (2): 159-67. https://doi.org/10.1007/s11630-006-0159-7.

2007. "Direct and Indirect Methods of Calculating Entropy Generation Rates in Turbulent Convective Heat Transfer Problems." Heat and Mass Transfer/Waerme- Und Stoffuebertragung 43 (3): 207-15. https://doi.org/10.1007/s00231-006-0086-x.

Herwig, Heinz. 2012. "The Role of Entropy Generation in Momentum and Heat Transfer." Journal of Heat Transfer 134 (3): 031003. https://doi.org/10.1115/1.4005128.

Herwig, Heinz, and Bastian Schmandt. 2014. "How to Determine Losses in a Flow Field: A Paradigm Shift Towards the Second Law Analysis.” Entropy 16 (6): 2959-89. https://doi.org/10.3390/e16062959.

Horlock, J. H. 2001. "The Basic Thermodynamics of Turbine Cooling.” Journal of Turbomachinery 123 (3): 583-92. https://doi.org/10.1115/1.1370156.

Horlock, J H, and L Torbidoni. 2006. "Turbine Blade Cooling: The Blade Temperature Distribution." Proceedings of the Institution of Mechanical Engineers, Part A: Journal of Power and Energy 220 (4): 343-53. https://doi.org/10.1243/09576509JPE177.

Jardine, Lachlan J., and Robert J. Miller. 2019. "The Effect of Heat Transfer on Turbine Performance." In Proceedings of the ASME Turbo Expo 2019: Power for Land, Sea and Air, 1-12.

Jones, W. P., and B. E. Launder. 1973. “The Calculation of Low-Reynolds-Number 
Phenomena with a Two-Equation Model of Turbulence." International Journal of Heat and Mass Transfer 16 (6): 1119-30. https://doi.org/10.1016/0017-9310(73)90125-7.

Keenan, Joseph H. 1951. "Availability and Irreversibility in Thermodynamics." British Journal of Applied Physics 2 (7): 183-92. https://doi.org/10.1088/0508-3443/2/7/302.

Launder, B. E., and B. I. Sharma. 1974. "Application of the Energy-Dissipation Model of Turbulence to the Calculation of Flow Near a Spinning Disc." Letters in Heat and Mass Transfer 1: 303-35.

Launder, B.E., and D. B. Spalding. 1974. "The Numerical Computation of Turbulent Flows." COMPUTER METHODS IN APPLIED MECHANICS AND ENGINEERING 3: 269-89.

Lees, Lester; Lin, Chia Chiao. 1946. "Investigation of the Stability of the Laminar Boundary Layer in a Compressible Fluid.” National Advisory Committee for Aeronautics. Vol. 1115. https://doi.org/10.1016/s0016-0032(22)90049-3.

Leggett, John, Aamir Shabbir, Edward Richardson, Vittorio Michelassi, Stephan Priebe, and Richard Sandberg. 2019. "Loss Analysis of Unsteady Turbomachinery Flows Based on the Mechanical Work Potential." Proceedings of the ASME Turbo Expo 2A-2019 (1): 113. https://doi.org/10.1115/GT2019-91253.

Li, Qiang, Philipp Schlatter, Luca Brandt, and Dan S. Henningson. 2009. "DNS of a Spatially Developing Turbulent Boundary Layer with Passive Scalar Transport." International Journal of Heat and Fluid Flow 30 (5): 916-29. https://doi.org/10.1016/j.ijheatfluidflow.2009.06.007.

Lim, Chia Hui, Graham Pullan, and John Northall. 2011. "Estimating the Loss Associated with Film Cooling for a Turbine Stage." Journal of Turbomachinery 134 (2): 1-10. https://doi.org/10.1115/1.4003255.

Lim, Shyang Maw, Anders Dahlkild, and Mihai Mihaescu. 2018. "Aerothermodynamics and Exergy Analysis in Radial Turbine With Heat Transfer." Journal of Turbomachinery 140 (9): 091007. https://doi.org/10.1115/1.4040852.

Lin, Ray-Sing, and Om P Sharma. 2014. "An Analytical Investigation on the Impact of Wall Cooling on the Development of Boundary Layers on the Suction Sides of Typical High Pressure Turbine Airfoils." Proceedings of ASME Turbo Expo 2014: Turbine Technical Conference and Exposition, 1-9. https://doi.org/10.1115/GT2014-26041.

Miller, Robert J. 2013. “Mechanical Work Potential.” In Proceedings of ASME Turbo Expo 2013: Power for Land, Sea and Air, 1-13.

Moran, M J, and E Sciubba. 1994. "Exergy Analysis: Principles and Practice.” Journal of Engineering for Gas Turbines and Power 116 (2): 285-90. https://doi.org/10.1115/1.2906818.

Phillips, Lee, and David Fyfe. 2011. "TURBID: A Routine for Generating Random Turbulent Inflow Data."

Poinsot, T. J., and S. K. Lelef. 1992. "Boundary Conditions for Direct Simulations of Compressible Viscous Flows.” Journal of Computational Physics 101 (1): 104-29. https://doi.org/10.1016/0021-9991(92)90046-2.

Roach, P. E., and D. H. Brierley. 1990. “The Influence of a Turbulent Freestream on Zero 
Pressure Gradient Transitional Boundary Layer Development, Part I: Test Cases T3A and T3b."

Schlichting, Hermann. 1968. Boundary-Layer Theory. McGraw-Hill, New York.

Sciubba, Enrico. 1997. “Calculating Entropy with CFD.” Mechanical Engineering 119 (10): $86-88$.

Shapiro, Ascher H., and W. R. Hawthorne. 1947. "The Mechanics and Thermodynamics of Steady One-Dimensional Gas Flows." Journal of Applied Mechanics.

Soderberg, C. R. 1949. "Unpublished Note.” Gas Turbine Laboratory, Massachusetts Institute of Technology.

Spalart, P. R., and S. R. Allmaras. 1992. "A One-Equation Turbulence Model For Aerodynamic Flows.” In 30th Aerospace Sciences Meeting and Exhibit. https://doi.org/10.2514/6.1992-439.

Standen, N. M. 1964. "A Concept of Mass Entrainment Applied to Compressible Turbulent Boundary Layers in Adverse Pressure Gradients." In Fourth Congress - Aeronautical Sciences, 1101-25.

Sutherland, William. 1893. "The Viscosity of Gases and Molecular Force." Philosophical Magazine Series 536 (223): 507-31. https://doi.org/10.1080/14786449308620508.

Tailliez, C., and A. Arntz. 2018. "CFD Assessment of the Use of Exergy Analysis for Losses Identification in Turbomachine Flows." 53rd 3AF International Conference on Applied Aerodynamics, no. March: 1-10.

https://www.researchgate.net/publication/324030010\%0ACFD.

Tam, Christopher K.W., and Jay C. Webb. 1993. "Dispersion-Relation-Preserving Finite Difference Schemes for Computational Acoustics." Journal of Computational Physics. https://doi.org/10.1006/jcph.1993.1142.

Traupel, Walter. 1982. Termische Turbomaschinen Zweiter Band Geländerte Betriebsbedingungen, Regelung,Mechanische Probleme, Temperaturprobleme. Springer-Verlag Berlin Heidelberg New York.

Trettel, Andrew, and Johan Larsson. 2016. "Mean Velocity Scaling for Compressible Wall Turbulence with Heat Transfer." Physics of Fluids 28. https://doi.org/10.1063/1.4942022.

Uysal, Selcuk Can, Eric Liese, Andrew C. Nix, and James Black. 2018. "A Thermodynamic Model to Quantify the Impact of Cooling Improvements on Gas Turbine Efficiency." Journal of Turbomachinery 140 (3): 1-11. https://doi.org/10.1115/1.4038614.

Walz, Alfred. 1967. "New General Law For the Turbulent Dissipation Integral." Physics of Fluids 10 (9). https://doi.org/10.1063/1.1762437.

Wenzel, Christoph, Björn Selent, Markus Kloker, and Ulrich Rist. 2018. "DNS of Compressible Turbulent Boundary Layers and Assessment of Data/Scaling-Law Quality." Journal of Fluid Mechanics 842 (October): 428-68. https://doi.org/10.1017/jfm.2018.179.

Wheeler, Andrew P.S., Anthony M.J. Dickens, and Robert J. Miller. 2018. "The Effect of Nonequilibrium Boundary Layers on Compressor Performance." Journal of 
Turbomachinery 140 (10): 1-10. https://doi.org/10.1115/1.4040094.

Wilcock, R. C., J. B. Young, and J. H. Horlock. 2005. "The Effect of Turbine Blade Cooling on the Cycle Efficiency of Gas Turbine Power Cycles." Journal of Engineering for Gas Turbines and Power 127 (1): 109. https://doi.org/10.1115/1.1805549.

Wilcox, David C. 1988. "Reassessment of the Scale-Determining Equation for Advanced Turbulence Models" 26 (11): 1299-1310. https://doi.org/10.2514/3.10041.

Wu, Xiaohua. 2017. "Inflow Turbulence Generation Methods." Annual Review of Fluid Mechanics 49 (1): 23-49. https://doi.org/10.1146/annurev-fluid-010816-060322.

Wu, Xiaohua, and Parviz Moin. 2009. "Direct Numerical Simulation of Turbulence in a Nominally Zero-Pressure-Gradient Flat-Plate Boundary Layer." Journal of Fluid Mechanics 630: 5-41. https://doi.org/10.1017/S0022112009006624.

Young, J. B., and John H. Horlock. 2006. "Defining the Efficiency of a Cooled Turbine." Journal of Turbomachinery 128 (4): 658-67. https://doi.org/10.1115/1.2218890.

Young, J. B., and R. C. Wilcock. 2002a. "Modeling the Air-Cooled Gas Turbine: Part 1General Thermodynamics." Journal of Turbomachinery 124 (2): 207-13. https://doi.org/10.1115/1.1415037.

- 2002b. "Modeling the Air-Cooled Gas Turbine: Part 2-Coolant Flows and Losses." Journal of Turbomachinery 124 (2): 214-21. https://doi.org/10.1115/1.1415038.

Zhang, You Sheng, Wei Tao Bi, Fazle Hussain, and Zhen Su She. 2013. "A Generalized Reynolds Analogy for Compressible Wall-Bounded Turbulent Flows." Journal of Fluid Mechanics 739: 392-420. https://doi.org/10.1017/jfm.2013.620.

Zhao, Yaomin, and Richard D. Sandberg. 2019. "Using a New Entropy Loss Analysis to Assess the Accuracy of RANS Predictions of an HPT Vane." Proceedings of the ASME Turbo Expo 2C-2019: 1-11. https://doi.org/10.1115/GT2019-90126.

Zlatinov, Metodi Blagoev, Choon Sooi Tan, Matthew Montgomery, Tito Islam, and Melissa Harris. 2012. "Turbine Hub and Shroud Sealing Flow Loss Mechanisms." Journal of Turbomachinery 134 (6): 1-12. https://doi.org/10.1115/1.4006294. 



\section{Appendix A General accounting methods}

\section{A.1 General flow work}

For a flow of single composition, the amount of work a device can extract is a combination of the kinetic energy and internal energy. By bringing the flow to rest with the reference environment, an ideal device could extract all the kinetic energy. By bringing the flow into mechanical and thermal equilibrium with the reference environment, an ideal device can only extract a fraction of the internal energy. The ideal flow work, $\mathbf{w}_{\mathbf{f}}$, is expressed in Equation A.1.

$$
\mathbf{w}_{\mathbf{f}}=\underbrace{h_{t}-h}_{\text {Kinetic energy }}+\underbrace{\int \eta_{t h} T d s+\int C_{p}^{e q} v d p}_{\text {Internal Energy }}
$$

The fraction of internal energy an ideal device can extract from a flow is set by the thermal efficiency, $\eta_{t h}$, and pressure equilibrium coefficient, $C_{p}^{e q}$, which are defined as:

$$
\eta_{t h}=1-\frac{T_{e x}}{T}, \quad C_{p}^{e q}=1-\frac{d p_{e x}}{d p}
$$

The thermal efficiency can be viewed as the efficiency of a cycle that only uses ideal devices and operates between an elemental sub-system and the reference environment. This is constrained to be less than unity by the second law of thermodynamics. The pressure equilibrium coefficient can be viewed as how the exhaust pressure varies. This is zero if the exhaust pressure varies with the system and unity if it varies with the reference environment.

By changing the thermal efficiency and pressure equilibrium coefficient, different general methods can be recovered. Consider the difference between a flow property and its closed form: 


$$
\mathbf{w}_{\mathbf{f}}=\mathbf{w}+\left(p-p_{e x}\right) v
$$

Multiply Equation A.3 by density and taking the partial derivative with time gives:

$$
\frac{\partial \mathbf{w}_{\mathbf{f}} \rho}{\partial t}=\frac{\partial \mathbf{w} \rho}{\partial t}+\frac{\partial\left(p-p_{e x}\right)}{\partial t}
$$

Substituting Equation A.3 into the LHS of Equation A.4 gives

$$
\frac{\partial \mathbf{w} \rho}{\partial t}+\nabla \cdot\left(\mathbf{w}_{\mathbf{f}} \rho \boldsymbol{V}\right)=\frac{\partial \mathbf{w}_{\mathbf{f}} \rho}{\partial t}+\nabla \cdot\left(\mathbf{w}_{\mathbf{f}} \rho \boldsymbol{V}\right)-\frac{\partial\left(p-p_{e x}\right)}{\partial t}
$$

Now wish to rewrite the right-hand side of Equation A.5 as the substantial derivative of $\mathbf{w}_{\mathbf{f}}$. First expand the first two terms on the right-hand side of Equation A.5 by the product rule.

$$
\frac{\partial \mathbf{w} \rho}{\partial t}+\nabla \cdot\left(\mathbf{w}_{\mathbf{f}} \rho \boldsymbol{V}\right)=\left[\rho \frac{\partial \mathbf{w}_{\mathbf{f}}}{\partial t}+\mathbf{w}_{\mathbf{f}} \frac{\partial \rho}{\partial t}+\rho \boldsymbol{V} \cdot \nabla \mathbf{w}_{\mathbf{f}}+\mathbf{w}_{\mathbf{f}} \nabla \cdot(\rho \boldsymbol{V})\right]-\frac{\partial\left(p-p_{e x}\right)}{\partial t}
$$

Rearranging terms in the square brackets gives:

$$
\begin{aligned}
& \frac{\partial \mathbf{w} \rho}{\partial t}+\nabla \cdot\left(\mathbf{w}_{\mathbf{f}} \rho \boldsymbol{V}\right) \\
& =\left[\rho\left(\frac{\partial \mathbf{w}_{\mathbf{f}}}{\partial t}+\boldsymbol{V} \cdot \nabla \mathbf{w}_{\mathbf{f}}\right)+\mathbf{w}_{\mathbf{f}}\left(\frac{\partial \rho}{\partial t}+\nabla \cdot(\rho \boldsymbol{V})\right)\right]-\frac{\partial\left(p-p_{e x}\right)}{\partial t}
\end{aligned}
$$

By continuity:

$$
\frac{\partial \rho}{\partial t}+\nabla \cdot(\rho \boldsymbol{V})=0
$$

Substituting Equation A.8 into Equation A.7 gives:

$$
\frac{\partial \mathbf{w} \rho}{\partial t}+\nabla \cdot\left(\mathbf{w}_{\mathbf{f}} \rho \boldsymbol{V}\right)=\rho \frac{D \mathbf{w}_{\mathbf{f}}}{D t}-\frac{\partial\left(p-p_{e x}\right)}{\partial t}
$$


Now need to find the substantial derivative of $\mathbf{w}_{\mathbf{f}}$. Substituting Equation A.1 into A.9:

$$
\frac{\partial \mathbf{w} \rho}{\partial t}+\nabla \cdot\left(\mathbf{w}_{\mathbf{f}} \rho \boldsymbol{V}\right)=\rho \frac{D\left(h_{t}-h\right)}{D t}+\eta_{t h} \rho T \frac{D s}{D t}+C_{p}^{e q} \frac{D p}{D t}-C_{p}^{e q} \frac{\partial p}{\partial t}
$$

Combining:

$$
\frac{\partial \mathbf{w} \rho}{\partial t}+\nabla \cdot\left(\mathbf{w}_{\mathbf{f}} \rho \boldsymbol{V}\right)=\underbrace{\rho \frac{D\left(h_{t}-h\right)}{D t}}_{\text {Kinetic energy }}+\eta_{t h} \underbrace{\rho T \frac{D s}{D t}}_{\text {Entropy Transport }}+C_{p}^{e q} \boldsymbol{V} \cdot \nabla p
$$

From the energy rate balance in differential form:

$$
\rho \frac{D\left(h_{t}-h\right)}{D t}=-\boldsymbol{V} \cdot \nabla p+\nabla \cdot[\overline{\overline{\boldsymbol{\tau}}} \cdot \boldsymbol{V}]-(\overline{\overline{\boldsymbol{\tau}}} \cdot \nabla) \cdot \boldsymbol{V}
$$

Examining the entropy transport equation:

$$
\rho T \frac{D s}{D t}=(\overline{\overline{\boldsymbol{\tau}}} \cdot \nabla) \cdot \boldsymbol{V}-\nabla \cdot \boldsymbol{q}+\dot{q}
$$

Substituting Equation A.12 and Equation A.13 into Equation A.11:

$$
\begin{aligned}
\frac{\partial \mathbf{w} \rho}{\partial t}+\nabla \cdot\left(\mathbf{w}_{\mathbf{f}} \rho \boldsymbol{V}\right)= & \underbrace{-\boldsymbol{V} \cdot \nabla p+\nabla \cdot[\overline{\overline{\boldsymbol{\tau}}} \cdot \boldsymbol{V}]-(\overline{\overline{\boldsymbol{\tau}}} \cdot \nabla) \cdot \boldsymbol{V}}_{\text {Kinetic energy }} \\
& \underbrace{+\eta_{t h}[(\overline{\overline{\boldsymbol{\tau}}} \cdot \nabla) \cdot \boldsymbol{V}-\nabla \cdot \boldsymbol{q}+\dot{q}]+C_{p}^{e q} \boldsymbol{V} \cdot \nabla p}_{\text {Internal energy }}
\end{aligned}
$$

Equation A.14 now becomes:

$$
\begin{aligned}
\frac{\partial \mathbf{w} \rho}{\partial t}+\nabla \cdot\left(\mathbf{w}_{\mathbf{f}} \rho \boldsymbol{V}\right) & =\nabla \cdot[\overline{\overline{\boldsymbol{\tau}}} \cdot \boldsymbol{V}] \\
& +\eta_{t h} \dot{q} \\
& -\eta_{t h} \nabla \cdot \boldsymbol{q} \\
& -\left(1-\eta_{t h}\right)[(\overline{\overline{\boldsymbol{\tau}}} \cdot \nabla) \cdot \boldsymbol{V}] \\
& -\left(1-C_{p}^{e q}\right) \boldsymbol{V} \cdot \nabla p
\end{aligned}
$$


To identify the loss mechanisms, the terms on the right-hand side should be split into terms that change the balance on the surfaces compared to the local volumetric mechanisms. This can be achieved by splitting the divergence of heat flux.

Equation A.16 demonstrates a unified equation for all general methods.

$$
\begin{aligned}
\frac{\partial \mathbf{w} \rho}{\partial t}+\nabla \cdot\left(\mathbf{w}_{\mathbf{f}} \rho \boldsymbol{V}\right) & =\nabla \cdot[\overline{\overline{\boldsymbol{\tau}}} \cdot \boldsymbol{V}] \\
& +\eta_{t h} \dot{q} \\
& -\nabla \cdot\left(\eta_{t h} \boldsymbol{q}\right) \\
& +\left(1-\eta_{t h}\right) \boldsymbol{q} \cdot \frac{\nabla \eta_{t h}}{1-\eta_{t h}} \\
& -\left(1-\eta_{t h}\right)[(\overline{\overline{\boldsymbol{\tau}}} \cdot \nabla) \cdot \boldsymbol{V}] \\
& -\left(1-C_{p}^{e q}\right) \boldsymbol{V} \cdot \nabla p
\end{aligned}
$$

The unified loss mechanisms for a general method are:

$$
\underbrace{\boldsymbol{q} \cdot \frac{\nabla \eta_{t h}}{1-\eta_{t h}}}_{\phi_{t h}}, \quad \underbrace{(\overline{\overline{\boldsymbol{\tau}}} \cdot \nabla) \cdot \boldsymbol{V}}_{\phi_{v}}, \quad \underbrace{\boldsymbol{V} \cdot \nabla p}_{\phi_{p}}
$$

where $\phi_{t h}$ is the thermal loss mechanism due to heat transfer across gradients in thermal efficiency, $\phi_{v}$ is the viscous loss mechanisms due to viscous dissipation, and $\phi_{p}$ is the loss due to communication between internal energy and kinetic energy.

By changing the thermal efficiency and pressure equilibrium coefficient, different general methods can be recovered. Some of these are tabulated in Table A.1: 
Table A.1 - Thermal efficiencies and pressure equilibrium coefficients for general methods.

\begin{tabular}{lcc}
\hline \hline & $\eta_{t h}$ & $C_{e q}^{p}$ \\
\hline Kinetic energy equation & $\eta_{t h}=0$ & $C_{p}^{e q}=0$ \\
Power-balance equation & $\eta_{t h}^{\mathrm{p}}=\left(1-\frac{p_{0}}{p}\right) \frac{\gamma-1}{\gamma}$ & $C_{p}^{e q}=1$ \\
Euergy equation & $\eta_{t h}^{\mathrm{m}}=1-\left(\frac{p_{0}}{p}\right)^{\frac{\gamma-1}{\gamma}}$ & $C_{p}^{e q}=1$ \\
Exergy equation & $\eta_{t h}^{\mathrm{e}}=1-\frac{T_{0}}{T}$ & $C_{p}^{e q}=1$ \\
Energy equation & $\eta_{t h}=1$ & $C_{p}^{e q}=1$ \\
\hline \hline
\end{tabular}

\section{A.2 The effect of real gases on the euergy method}

The effect of real gases is to change how the euergy thermal efficiency is calculated. This is calculated based upon the ideal work that could be extracted by a reversible, adiabatic expansion to a constant pressure. This can be expressed as:

$$
\eta_{t h}^{\mathrm{m}}=1-\frac{T_{s, p_{0}}}{T}=1-\left(\frac{p_{0}}{p}\right)^{\frac{\gamma-1 \beta_{T} p}{\gamma \beta_{p} T}}
$$

Table A.2 - Gas compression parameters.

\begin{tabular}{lcc}
\hline \hline & Definition & Ideal gas \\
\hline Compressibility factor & $Z=f(T, p)$ & $Z=1$ \\
Isothermal compressibility & $\beta_{T}=-\frac{1}{v}\left(\frac{\partial v}{\partial p}\right)_{T}=\frac{1}{p}-\frac{1}{Z}\left(\frac{\partial Z}{\partial p}\right)_{T}$ & $\beta_{T}=\frac{1}{p}$ \\
$\begin{array}{l}\text { Isobaric compressibility } \\
\text { (thermal expansion coefficient) }\end{array}$ & $\beta_{p}=\frac{1}{v}\left(\frac{\partial v}{\partial T}\right)_{p}=\frac{1}{T}-\frac{1}{Z}\left(\frac{\partial Z}{\partial T}\right)_{p}$ & $\beta_{p}=\frac{1}{T}$ \\
\hline
\end{tabular}




\section{A.3 Comparing the euergy and power-balance methods}

Here, the assumptions with which the euergy equation becomes the power-balance method will be demonstrated. Examining Equation A.11:

$$
\left[\frac{\partial \mathbf{m} \rho}{\partial t}+\nabla \cdot\left(\mathbf{m}_{\mathbf{f}} \rho \boldsymbol{V}\right)\right]-\left[\frac{\partial \mathbf{p} \rho}{\partial t}+\nabla \cdot\left(\mathbf{p}_{\mathbf{f}} \rho \boldsymbol{V}\right)\right]=\left(\eta_{t h}^{\mathbf{m}}-\eta_{t h}^{\mathbf{p}}\right) \rho T \frac{D s}{D t}
$$

The key difference is how they value the transport of entropy. This indicated that the two methods are the same when:

1. $\eta_{t h}^{\mathbf{m}} \approx \eta_{t h}^{\mathbf{p}} \rightarrow p \approx p_{0}$

2. The transfer and generation of entropy is small, (i.e. highly aerodynamic devices with no heat transfer).

3. The entropy balance is steady.

Using Gibbs' equation, the euergy equation can be written as:

$$
\frac{\partial \mathbf{m} \rho}{\partial t}+\nabla \cdot\left(\mathbf{m}_{\mathbf{f}} \rho \boldsymbol{V}\right)=\left[\rho \frac{D h_{t}}{D t}-\frac{\partial p}{\partial t}\right]-\left(1-\eta_{t h}^{\mathbf{m}}\right) \rho T \frac{D s}{D t}
$$

The focus will now be on how the transport of entropy changes:

$$
\begin{aligned}
\rho T \frac{D s}{D t} & =\rho \frac{D h_{t}}{D t}-\frac{D p}{D t}-\rho \frac{D\left(h_{t}-h\right)}{D t} \\
& =\left[\rho \frac{D h_{t}}{D t}-\frac{\partial p}{\partial t}\right]-\boldsymbol{V} \cdot \nabla p-\rho \boldsymbol{V} \cdot \nabla\left(h_{t}-h\right)-\rho \frac{\partial\left(h_{t}-h\right)}{\partial t}
\end{aligned}
$$

Examining the second term on the right-hand side and introducing a constant Trefftz pressure term:

$$
\begin{aligned}
\boldsymbol{V} \cdot \nabla p=\boldsymbol{V} \cdot \nabla & p-\boldsymbol{V} \cdot \nabla p_{\text {Trefftz }} \\
& =\nabla \cdot\left(\boldsymbol{V}\left[p-p_{\text {Trefftz }}\right]\right)-\left[p-p_{\text {Trefftz }}\right](\nabla \cdot \boldsymbol{V})
\end{aligned}
$$

Examining the third term on the right-hand side and introducing a constant Trefftz velocity term: 


$$
\begin{aligned}
\rho \boldsymbol{V} \cdot \nabla\left(h_{t}-h\right) & =\rho \boldsymbol{V} \cdot \nabla\left(\frac{V^{2}}{2}\right)-\rho \boldsymbol{V} \cdot \nabla\left(\frac{V_{\text {Trefftz }}^{2}}{2}\right) \\
& =\nabla \cdot\left(\rho \boldsymbol{V} \frac{V^{2}-V_{\text {Trefftz }}^{2}}{2}\right)-\left(\frac{V^{2}-V_{\text {Trefftz }}^{2}}{2}\right)(\nabla \cdot \rho \boldsymbol{V})
\end{aligned}
$$

Substituting Equation A.22 and Equation A.23 into Equation A.21 and applying continuity from Equation A.8:

$$
\begin{array}{r}
\rho T \frac{D s}{D t}=\left[\rho \frac{D h_{t}}{D t}-\frac{\partial p}{\partial t}\right]-\nabla \cdot\left(\rho \boldsymbol{V}\left[\frac{p-p_{\text {Trefftz }}}{\rho}+\frac{V^{2}-V_{\text {Trefftz }}^{2}}{2}\right]\right) \\
+\left[p-p_{\text {Trefftz }}\right](\nabla \cdot \boldsymbol{V})-\left(h_{t}-h\right) \frac{\partial \rho}{\partial t}-\rho \frac{\partial\left(h_{t}-h\right)}{\partial t}
\end{array}
$$

The second term on the right is the source term identified by Drela (2009). Setting $p_{\text {Trefftz }}=p_{0}$ and $V_{\text {Trefftz }}=0$, this becomes:

$$
\frac{p-p_{0}}{\rho}+\frac{1}{2} V^{2}=\left(1-\frac{p_{0}}{p}\right) \frac{p}{\rho}+\frac{1}{2} V^{2}=\underbrace{\left[\left(1-\frac{p_{0}}{p}\right) \frac{\gamma-1}{\gamma}\right]}_{\eta_{t h}^{\mathbf{p}}} h+\frac{1}{2} V^{2}=\mathbf{p}_{\mathbf{f}}
$$

Substituting Equation A.25 into Equation A.24:

$$
\rho T \frac{D s}{D t}=\left[\rho \frac{D h_{t}}{D t}-\frac{\partial p}{\partial t}\right]-\nabla \cdot\left(\mathbf{p}_{\mathbf{f}} \rho \boldsymbol{V}\right)+\left[p-p_{0}\right](\nabla \cdot \boldsymbol{V})-\frac{\partial\left(\rho\left(h_{t}-h\right)\right)}{\partial t}
$$

Substituting Equation A.26 into Equation A.20:

$$
\begin{aligned}
\frac{\partial \mathbf{m} \rho}{\partial t}+\nabla \cdot\left(\mathbf{m}_{\mathbf{f}} \rho \boldsymbol{V}\right) & \\
& =\eta_{t h}^{\mathbf{m}}\left[\rho \frac{D h_{t}}{D t}-\frac{\partial p}{\partial t}\right] \\
& -\left(1-\eta_{t h}^{\mathbf{m}}\right)\left[\left[p-p_{0}\right](\nabla \cdot \boldsymbol{V})-\nabla \cdot\left(\mathbf{p}_{\mathbf{f}} \rho \boldsymbol{V}\right)-\frac{\partial\left(\rho\left(h_{t}-h\right)\right)}{\partial t}\right]
\end{aligned}
$$


Assuming steady flow, Equation A.27 becomes:

$$
\begin{aligned}
\nabla \cdot\left(\mathbf{m}_{\mathbf{f}} \rho \boldsymbol{V}\right)- & \nabla \cdot\left(\mathbf{p}_{\mathbf{f}} \rho \boldsymbol{V}\right) \\
& =\eta_{t h}^{\mathbf{m}}\left(\left[\nabla \cdot\left(h_{t} \rho \boldsymbol{V}\right)\right]-\nabla \cdot\left(\mathbf{p}_{\mathbf{f}} \rho \boldsymbol{V}\right)\right)-\left(1-\eta_{t h}^{\mathbf{m}}\right)\left[p-p_{0}\right](\nabla \cdot \boldsymbol{V})
\end{aligned}
$$

There are three assumptions required for the power-balance method to equal euergy. At low thermal efficiencies, the term that is causing the difference is the divergence of velocity. The assumptions are:

1. $\eta_{t h}^{\mathrm{m}} \approx 0$ or equivalently $p \approx p_{0}$.

2. $\nabla \cdot \boldsymbol{V} \approx 0$ which implies incompressible, adiabatic flow.

3. Steady flow.

\section{A.4 Comparing the euergy method and stagnation pressure loss}

Starting from Equation A.20, first the term of interest will be added and subtracted:

$$
\begin{aligned}
& \frac{\partial \mathbf{m} \rho}{\partial t}+\nabla \cdot\left(\mathbf{m}_{\mathbf{f}} \rho \boldsymbol{V}\right)=\eta_{t h}^{\mathbf{m}}\left[\rho \frac{D h_{t}}{D t}-\frac{D p}{D t}\right] \\
& +\left(1-\eta_{t h}^{\mathbf{m}}\right)\left[\frac{\nabla \cdot\left(\rho \boldsymbol{V}\left(p_{t}-p_{0}\right)\right)}{\rho}\right] \\
& +\left(1-\eta_{t h}^{\mathbf{m}}\right)\left[\rho \frac{D\left(h_{t}-h\right)}{D t}-\frac{\nabla \cdot\left(\rho \boldsymbol{V}\left(p_{t}-p_{0}\right)\right)}{\rho}+\boldsymbol{V} \cdot \nabla p\right]
\end{aligned}
$$

Examining the final term of Equation A.29:

$$
\begin{aligned}
& \rho \frac{D\left(h_{t}-h\right)}{D t}-\frac{\nabla \cdot\left(\rho \boldsymbol{V}\left(p_{t}-p_{0}\right)\right)}{\rho}+\boldsymbol{V} \cdot \nabla p \\
& =\rho \frac{D\left(h_{t}-h\right)}{D t}-\boldsymbol{V} \cdot \nabla\left(p_{t}-p_{d}\right)+\boldsymbol{V} \cdot \nabla p-\frac{p_{t}-p_{d}}{\rho} \nabla \cdot \rho \boldsymbol{V} \\
& =\rho \frac{D\left(h_{t}-h\right)}{D t}-\frac{D\left(p_{t}-p\right)}{D t}+\frac{\partial\left(p_{t}-p\right)}{\partial t}+\frac{p_{t}-p_{0}}{\rho} \frac{\partial \rho}{\partial t} \\
& =-\left(1-\frac{\rho}{\rho_{t}}\right) \frac{D p_{t}}{D t}+\frac{\partial\left(p_{t}-p\right)}{\partial t}+\frac{p_{t}-p_{0}}{\rho} \frac{\partial \rho}{\partial t}
\end{aligned}
$$




$$
\begin{aligned}
& \frac{\partial \mathbf{m} \rho}{\partial t}+\nabla \cdot\left(\mathbf{m}_{\mathbf{f}} \rho \boldsymbol{V}\right)=\eta_{t h}^{\mathbf{m}}\left[\rho \frac{D h_{t}}{D t}-\frac{D p}{D t}\right] \\
& +\left(1-\eta_{t h}^{\mathbf{m}}\right)\left[\frac{\nabla \cdot\left(\rho \boldsymbol{V}\left(p_{t}-p_{0}\right)\right)}{\rho}\right] \\
& +\left(1-\eta_{t h}^{\mathbf{m}}\right)\left[\frac{\partial\left(p_{t}-p\right)}{\partial t}+\frac{p_{t}-p_{0}}{\rho} \frac{\partial \rho}{\partial t}-\left(1-\frac{\rho}{\rho_{t}}\right) \frac{D p_{t}}{D t}\right]
\end{aligned}
$$

For steady flow:

$$
\begin{aligned}
\nabla \cdot\left(\mathbf{m}_{\mathbf{f}} \rho \boldsymbol{V}\right)-\frac{\nabla \cdot\left(\rho \boldsymbol{V}\left(p_{t}-p_{0}\right)\right)}{\rho} & =\eta_{t h}^{\mathbf{m}}\left(\left[\rho \frac{D h_{t}}{D t}-\frac{D p}{D t}\right]-\frac{\nabla \cdot\left(\rho \boldsymbol{V}\left(p_{t}-p_{0}\right)\right)}{\rho}\right) \\
& -\left(1-\eta_{t h}^{\mathbf{m}}\right)\left[\left(1-\frac{\rho}{\rho_{t}}\right) V \cdot \nabla p_{t}\right]
\end{aligned}
$$

Examining the final term, this disappears at low Mach number:

$$
1-\frac{\rho}{\rho_{t}}=1-\left(1+\frac{\gamma-1}{2} M^{2}\right)^{-\frac{1}{\gamma-1}}=\frac{\mathrm{M}^{2}}{2}+\frac{\gamma \mathrm{M}^{4}}{8}+O\left(\mathrm{M}^{6}\right)
$$

The assumptions are:

1. $\eta_{t h}^{\mathrm{m}} \approx 0 \rightarrow p \approx p_{0}$ or flows with small changes in kinetic energy and no heat/work input.

2. $V \cdot \nabla p_{t} \approx 0$ or low Mach number

3. Steady flow.

To examine a generalizable form of the stagnation pressure loss coefficient, take the incompressible form of stagnation pressure loss:

$$
\begin{aligned}
\frac{p_{t}-p_{0}}{\rho} & =\left[\left(1+\frac{\gamma-1}{2} \mathrm{M}^{2}\right)^{\frac{\gamma}{\gamma-1}}-\frac{p_{0}}{p}\right] \frac{\gamma-1}{\gamma} h \\
& =\left[\left(1+\frac{\gamma-1}{2} \mathrm{M}^{2}\right)^{\frac{\gamma}{\gamma-1}}-\frac{\gamma}{2} \mathrm{M}^{2}-\frac{p_{0}}{p}\right] \frac{\gamma-1}{\gamma} h+\frac{V^{2}}{2}
\end{aligned}
$$

For low Mach number,

$$
\left(1+\frac{\gamma-1}{2} \mathrm{M}^{2}\right)^{\frac{\gamma}{\gamma-1}}=\left[1+\frac{\gamma}{2} \mathrm{M}^{2}+\frac{\gamma}{8} \mathrm{M}^{4}+O\left(\mathrm{M}^{6}\right)\right]
$$


Now assuming that the enthalpy deviations scale with the total enthalpy (assuming adiabatic flow):

$$
\frac{p_{t}-p_{0}}{\rho}=\underbrace{\left[1-\frac{p_{0}}{p}+\frac{\gamma}{8} \mathrm{M}^{4}+O\left(\mathrm{M}^{6}\right)\right] \frac{\gamma-1}{\gamma}}_{\eta_{t h}^{\mathrm{y}}} h+\frac{V^{2}}{2}
$$




\section{Appendix B Trailing edge loss}

\section{B.1 Kinetic energy loss coefficient decomposition}

To examine the downstream control volume, $\mathrm{CV}_{2}$, in more detail, the schematic presented below will be used. This provides the framework, but to simplify the mathematics the angles have been assumed to be small, $\alpha=\beta=0$.

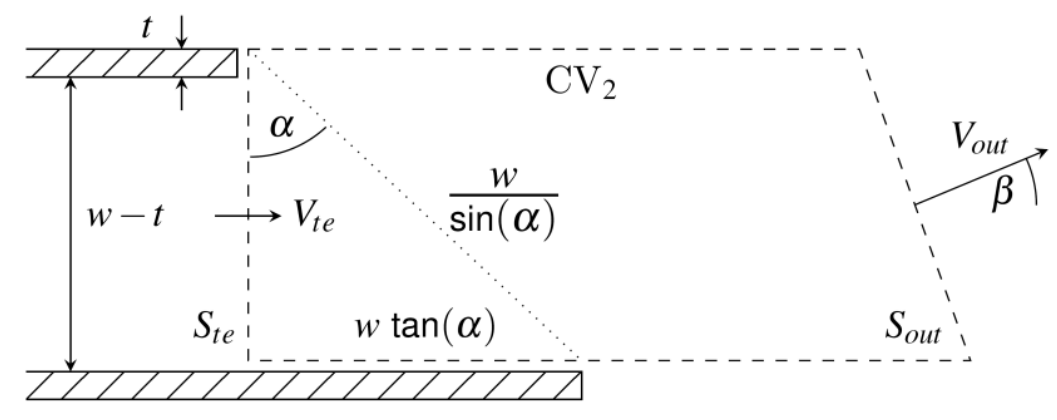

Fig. B.1 - Schematic of the control volume examining the trailing edge flow.

Consider the change in kinetic energy flux per unit depth across control volume $\mathrm{CV}_{2}$ :

$$
\begin{aligned}
& \epsilon=\iint_{S_{t e}} \rho \boldsymbol{V} \frac{V^{2}}{2} d S-\iint_{S_{\text {out }}} \rho \boldsymbol{V} \frac{V^{2}}{2} d S \\
& =\dot{m} \frac{V_{t e}^{2}}{2}-\rho_{t e} V_{t e} \frac{V_{t e}^{2}}{2} \delta_{3, t e}-\dot{m} \frac{V_{o u t}^{2}}{2} \\
& =\rho_{t e} V_{t e}\left[\frac{V_{t e}^{2}}{2}-\frac{V_{o u t}^{2}}{2}\right]\left(w-t-\delta_{1, t e}\right)-\rho_{t e} V_{t e} \frac{V_{t e}^{2}}{2} \delta_{3, t e} \\
& =\frac{\rho_{t e} V_{t e}^{3}}{2}\left\{\left[1-\frac{V_{o u t}^{2}}{V_{t e}^{2}}\right]\left(w-t-\delta_{1, t e}\right)-\delta_{3, t e}\right\}
\end{aligned}
$$

Normalising Equation B.37 by flow at the trailing edge: 


$$
\omega_{T E}^{K E^{\prime}}=\frac{\epsilon}{0.5 \rho_{t e} V_{t e}^{3} w}=\left[1-\frac{V_{o u t}^{2}}{V_{t e}^{2}}\right] \frac{\left(w-t-\delta_{1, t e}\right)}{w}-\frac{\delta_{3, t e}}{w}
$$

This is a simple form but is not most useful form for design. The aim now is to express this loss coefficient in terms of boundary layer and design parameters. Examining the first term in Equation B.38:

$$
\begin{aligned}
{\left[1-\frac{V_{\text {out }}^{2}}{V_{\text {te }}^{2}}\right] } & =\underbrace{\left(1-\frac{V_{\text {out }}}{V_{\text {te }}}\right)}_{A}\left(1+\frac{V_{\text {out }}}{V_{\text {te }}}\right) \\
& =A(2-A) \\
& =2 A-A^{2}
\end{aligned}
$$

The term A shown in Equation B.39 can be expressed in two ways. First, using conversation of momentum in the $\mathrm{x}$-direction:

$$
\begin{aligned}
& \rho_{t e} V_{t e}^{2}\left(w-t-\delta_{1, t e}\right)+p_{t e}(w-t)+p_{b} t-\rho_{t e} V_{t e}^{2} \delta_{2, t e}=\rho_{\text {out }} V_{\text {out }}^{2} w+p_{\text {out }} w \\
& \dot{m} V_{\text {te }}\left(1-\frac{V_{2}}{V_{t e}}\right)=\dot{m} V_{t e} \frac{\delta_{2, t e}}{w-t-\delta_{1, t e}}-\left(p_{t e}-p_{\text {out }}\right)(w-t)-\left(p_{b}-p_{\text {out }}\right) t \\
& \left(1-\frac{V_{\text {out }}}{V_{\text {te }}}\right)=\frac{\delta_{2, t e}}{w-t-\delta_{1 t e}}-\frac{p_{t e}-p_{\text {out }}}{\rho_{\text {te }} V_{\text {te }}^{2}} \frac{w-t}{w-t-\delta_{1 t e}}-\frac{p_{b}-p_{\text {out }}}{\rho_{\text {te }} V_{\text {te }}^{2}} \frac{t}{w-t-\delta_{1 t e}}
\end{aligned}
$$

Secondly, using continuity:

$$
\begin{aligned}
\rho_{\text {te }} V_{\text {te }}\left(w-t-\delta_{1, t e}\right)=\rho_{\text {out }} V_{\text {out }} w \\
1-\frac{V_{\text {out }}}{V_{\text {te }}}=1-\frac{\rho_{\text {te }}}{\rho_{\text {out }}} \frac{w-t-\delta_{1, t e}}{w} \\
=\left(1-\frac{\rho_{\text {te }}}{\rho_{\text {out }}}\right)+\frac{\rho_{\text {te }}}{\rho_{\text {out }}} \frac{t+\delta_{1, t e}}{w}
\end{aligned}
$$

Equation B.41 can be simplified by assuming the flow is approximately incompressible (i.e. $\frac{\rho_{\text {te }}}{\rho_{\text {out }}} \approx 1$ ): 


$$
\left(1-\frac{V_{\text {out }}}{V_{\text {te }}}\right)^{2} \approx\left(\frac{t+\delta_{1, t e}}{w}\right)^{2}
$$

Substituting Equations B.40 and B.42 into B.39, and then substituting this into B.38, the loss coefficient can be expressed using flow parameters. The algebra has been simplified by assuming $\frac{1}{w-t-\delta_{1, t e}} \approx \frac{1}{w}$ :

$$
\begin{aligned}
\omega_{T E}^{K E^{\prime}} & \approx \frac{2 \delta_{2, t e}}{w}-\frac{p_{t e}-p_{\text {out }}}{0.5 \rho_{\text {te }} V_{\text {te }}^{2}} \frac{w-t}{w}-\frac{p_{b}-p_{\text {out }}}{0.5 \rho_{\text {te }} V_{\text {te }}^{2}} \frac{t}{w}-\frac{\delta_{3, t e}}{w}+\left(\frac{t+\delta_{1, t e}}{w}\right)^{2} \\
& =\frac{\delta_{3, t e}}{w}\left[\frac{2}{H_{32, t e}}-1\right]-\frac{p_{t e}-p_{\text {out }}}{0.5 \rho_{\text {te }} V_{\text {te }}^{2}} \frac{w-t}{w}-\frac{p_{b}-p_{\text {out }}}{0.5 \rho_{\text {te }} V_{\text {te }}^{2}} \frac{t}{w}+\left(\frac{t+\delta_{1, t e}}{w}\right)^{2}
\end{aligned}
$$

The trailing edge pressure is typically similar to the downstream pressure $\left(p_{t e} \approx p_{\text {out }}\right)$. This leads to the second term in Equation B.43 being small.

Defining a base pressure coefficient:

$$
c_{p b}=\frac{p_{b}-p_{\text {out }}}{0.5 \rho_{t e} V_{t e}^{2}}
$$

Substituting Equation B.44 into Equation B.43 recovers the trailing edge loss decomposition presented by Denton, except $\delta_{3, t e}$ has replaced $\delta_{1, t e}$ :

$$
\omega_{T E}^{K E^{\prime}} \approx \frac{\delta_{3, t e}}{w}\left[\frac{2}{H_{32, t e}}-1\right]-c_{p b} \frac{t}{w}+\left(\frac{t+\delta_{1, t e}}{w}\right)^{2}
$$

If the dissipation that occurs in the upstream control volume is included, then Denton's mixing loss breakdown is recovered. It is worth emphasising that this expression is only valid for relatively thin trailing edges and incompressible flow.

If the trailing edge thickness is very thin (as in the case of external aerodynamics), then the term for thin trailing edges presented by Drela (2009) is recovered. 


\section{B.2 Transonic trailing edge loss}

For a transonic turbine blade, the mass flow rate fixed by the compressible function. Often the blades are choked at the throat:

$$
\dot{m} \frac{\sqrt{c_{p} T_{t}}}{(w-t) p_{t, \text { throat }}}=F(1)=\text { constant }
$$

Assuming adiabatic flow, the stagnation enthalpy will be constant. Specifying the mixed-out Mach number, $\mathrm{M}_{\text {out }}$, then:

$$
\dot{m} \frac{\sqrt{c_{p} T_{t}}}{w p_{t, \text { out }}}=F\left(\mathrm{M}_{\text {out }}\right)=\text { constant }
$$

Dividing Equation B.46 by Equation B.47 gives:

$$
\frac{p_{t, \text { out }}}{p_{t, \text { throat }}}=\frac{w-t}{w} \frac{F(1)}{F\left(\mathrm{M}_{\text {out }}\right)}
$$

Considering the change in euergy through this system:

$$
\omega_{T E}^{\mathbf{m}}=\frac{\iint_{S_{\text {throat }}} \rho \boldsymbol{V}\left(1-\left(\frac{p_{0}}{p_{t}}\right)^{\frac{\gamma-1}{\gamma}}\right) h_{t} d S}{\iint_{S_{\text {out }}} \rho \boldsymbol{V} \frac{V^{2}}{2} d S}-\frac{\iint_{S_{\text {out }}} \rho \boldsymbol{V}\left(1-\left(\frac{p_{0}}{p_{t}}\right)^{\frac{\gamma-1}{\gamma}}\right) h_{t} d S}{\iint_{S_{\text {out }}} \rho \boldsymbol{V} \frac{V^{2}}{2} d S}
$$

Assuming the total pressure is almost uniform across the channel, Equation B.49 becomes: 


$$
\begin{aligned}
\omega_{T E}^{\mathrm{m}}=\left[1-\left(\frac{p_{0}}{p_{t, \text { throat }}}\right)^{\frac{\gamma-1}{\gamma}}\right] \frac{\iint_{S_{\text {throat }}} \rho \boldsymbol{V} h_{t} d S}{\iint_{S_{\text {out }}} \rho \boldsymbol{V} \frac{V^{2}}{2} d S} \\
-\left[1-\left(\frac{p_{0}}{p_{t, \text { out }}}\right)^{\frac{\gamma-1}{\gamma}}\right] \frac{\iint_{S_{\text {out }}} \rho \boldsymbol{V} h_{t} d S}{\iint_{S_{\text {out }}} \rho \boldsymbol{V} \frac{V^{2}}{2} d S}
\end{aligned}
$$

For adiabatic single-stream flow, the total enthalpy is constant, the two integrals are equal. This assumes that there is no heat transfer leaving the control volume $\left(\mathrm{CV}_{2}\right)$, but there could be in the blade control volume $\left(\mathrm{CV}_{1}\right)$. Since the downstream plane is easier to measure, this will be retained. Equation B.50 becomes:

$$
\begin{aligned}
\omega_{T E}^{\mathbf{m}} & =\left(\left(\frac{p_{0}}{p_{t, \text { out }}}\right)^{\frac{\gamma-1}{\gamma}}-\left(\frac{p_{0}}{p_{t, \text { throat }}}\right)^{\frac{\gamma-1}{\gamma}}\right) \frac{\iint_{S_{\text {out }}} \rho \boldsymbol{V} h_{t} d S}{\iint_{S_{\text {out }}} \rho \boldsymbol{V} \frac{V^{2}}{2} d S} \\
& =\left(\frac{p_{0}}{p_{t, \text { out }}}\right)^{\frac{\gamma-1}{\gamma}}\left(1-\left(\frac{p_{t, \text { out }}}{p_{t, \text { throat }}}\right)^{\frac{\gamma-1}{\gamma}}\right) \frac{\iint_{S_{\text {out }}} \rho \boldsymbol{V} h_{t} d S}{\iint_{S_{\text {out }}} \rho \boldsymbol{V} \frac{V^{2}}{2} d S}
\end{aligned}
$$

If the reference environment pressure is taken to be that of the downstream static pressure, $p_{0}=p_{\text {out }}$, then all the pressure ratios become functions of Mach number:

$$
\omega_{T E}^{\mathrm{m}}=\frac{1}{\left(1+\frac{\gamma-1}{2} \mathrm{M}_{\text {out }}^{2}\right)}\left(1-\left(\frac{w-t}{w} \frac{F(1)}{F\left(\mathrm{M}_{\text {out }}\right)}\right)^{\frac{\gamma-1}{\gamma}}\right) \frac{\iint_{S_{\text {out }}} \rho \boldsymbol{V} h_{t} d S}{\iint_{S_{\text {out }}} \rho \boldsymbol{V} \frac{V^{2}}{2} d S}
$$

The trailing edge loss is now represented by a change in the exit Mach number, $\mathrm{M}_{\text {out }}$.

\section{B.3 Linking the euergy loss coefficient to kinetic energy loss}

Considering the change in euergy across the control volume, $\mathrm{CV}_{2}$, and assuming the static pressure is constant across the flow at both the trailing edge and downstream: 


$$
\begin{gathered}
\omega_{T E}^{\mathbf{m}}=\left(1-\left(\frac{p_{0}}{p_{t e}}\right)^{\frac{\gamma-1}{\gamma}}\right) \frac{\iint_{S_{\text {te }}} \rho \boldsymbol{V} h d S}{\iint_{S_{\text {out }}} \rho \boldsymbol{V} \frac{V^{2}}{2} d S}-\left(1-\left(\frac{p_{0}}{p_{\text {out }}}\right)^{\frac{\gamma-1}{\gamma}}\right) \frac{\iint_{S_{\text {out }}} \rho \boldsymbol{V} h d S}{\iint_{S_{\text {out }}} \rho \boldsymbol{V} \frac{V^{2}}{2} d S} \\
+\frac{\iint_{S_{\text {te }}} \rho \boldsymbol{V} \frac{V^{2}}{2} d S}{\iint_{S_{\text {out }}} \rho \boldsymbol{V} \frac{V^{2}}{2} d S}-\frac{\iint_{S_{\text {out }}} \rho \boldsymbol{V} \frac{V^{2}}{2} d S}{\iint_{S_{\text {out }}} \rho \boldsymbol{V} \frac{V^{2}}{2} d S}
\end{gathered}
$$

If the downstream control volume is adiabatic, Equation B.53 becomes:

$$
\begin{array}{r}
\omega_{T E}^{\mathrm{m}}=\left(\left(\frac{p_{0}}{p_{\text {out }}}\right)^{\frac{\gamma-1}{\gamma}}-\left(\frac{p_{0}}{p_{\text {te }}}\right)^{\frac{\gamma-1}{\gamma}}\right)\left(\frac{\iint_{S_{\text {out }}} \rho \boldsymbol{V} h_{t} d S-\iint_{S_{\text {out }}} \rho \boldsymbol{V} \frac{V^{2}}{2} d S}{\iint_{S_{\text {out }}} \rho \boldsymbol{V} \frac{V^{2}}{2} d S}\right) \\
+\left(\frac{p_{0}}{p_{\text {te }}}\right)^{\frac{\gamma-1}{\gamma}}\left(\frac{\iint_{S_{\text {te }}} \rho \boldsymbol{V} \frac{V^{2}}{2} d S-\iint_{S_{\text {out }}} \rho \boldsymbol{V} \frac{V^{2}}{2} d S}{\iint_{S_{\text {out }}} \rho \boldsymbol{V} \frac{V^{2}}{2} d S}\right)
\end{array}
$$

Representing Equation B.54 using integral boundary layer parameters:

$$
\begin{gathered}
\omega_{T E}^{\mathrm{m}}=\left(\left(\frac{p_{0}}{p_{\text {out }}}\right)^{\frac{\gamma-1}{\gamma}}-\left(\frac{p_{0}}{p_{t e}}\right)^{\frac{\gamma-1}{\gamma}}\right)\left(\frac{w-t-\delta_{3, t e}-\left(1-\frac{h_{w}}{h_{t e}}\right) \delta_{H, t e}}{w}\right) \frac{2 h_{t e}}{V_{\text {te }}^{2}} \frac{V_{t e}^{2}}{V_{\text {out }}^{2}} \\
+\left(\frac{p_{0}}{p_{t e}}\right)^{\frac{\gamma-1}{\gamma}} \omega_{T E}^{K E}
\end{gathered}
$$

where:

$$
\delta_{H}=\int \frac{\rho \boldsymbol{V}}{\rho_{m} V_{m}}\left(1-\frac{h-h_{w}}{h_{m}-h_{w}}\right) d y
$$

If the reference environment pressure is equal to the downstream pressure, $p_{0}=p_{\text {out }}$, then: 


$$
\omega_{T E}^{\mathbf{m}^{\prime}} \approx\left(1-\left(\frac{p_{0}}{p_{t e}}\right)^{\frac{\gamma-1}{\gamma}}\right)\left(1+\left(\frac{h_{w}}{h_{t e}}-1\right) \frac{\delta_{H, t e}}{w}\right) \frac{2 h_{t e}}{V_{t e}^{2}} \frac{V_{t e}^{2}}{V_{o u t}^{2}}+\omega_{T E}^{K E}
$$

If this pressure is also the same as the pressure in the mainstream at the trailing edge, $p_{0}=p_{\text {te }}=p_{\text {out }}$, then:

$$
\omega_{T E}^{\mathbf{m}}=\omega_{T E}^{\mathbf{p}}=\omega_{T E}^{K E}
$$

If, in addition, the density across the downstream control volume is constant, $\rho_{\text {out }}=$ $\rho_{\text {te }}$, then:

$$
\omega_{T E}^{\mathbf{m}}=\omega_{T E}^{\mathbf{p}}=\omega_{T E}^{K E}=\omega_{T E}^{p}
$$







\section{Appendix C Boundary layer analysis}

\section{C.1 Integral boundary layer method}

The aim is to determine a relationship between dissipation coefficient and wall temperature ratio. To do this, a dummy coordinate system will be used based on a modified Stewartson transformation:

$$
d Y=\frac{\rho a_{m}}{\rho_{t} a_{t}} d y, \quad U=\frac{a_{t}}{a_{m}} u
$$

First looking at momentum thickness, $\delta_{2}$ :

$$
\delta_{2}=\int \frac{\rho u}{\rho_{m} u_{m}}\left(1-\frac{u}{u_{m}}\right) d y
$$

Transforming coordinate system:

$$
\begin{aligned}
\delta_{2} & =\frac{\rho_{t} a_{t}}{\rho_{m} a_{m}} \int \frac{U}{U_{m}}\left(1-\frac{U}{U_{m}}\right) d Y \\
& =\left(1+\frac{\gamma-1}{2} M_{m}^{2}\right)^{\frac{\gamma+1}{2(\gamma-1)}} \delta_{2, t r} \\
& =\left(\frac{T_{t}}{T_{e}}\right)^{\frac{\gamma+1}{2(\gamma-1)}} \delta_{2, t r}
\end{aligned}
$$

Now investigating mean kinetic energy thickness, $\delta_{3}$ :

$$
\delta_{3}=\int \frac{\rho u}{\rho_{m} u_{m}}\left(1-\left(\frac{u}{u_{m}}\right)^{2}\right) d y
$$

Transforming coordinate system:

$$
\delta_{3}=\frac{\rho_{t} a_{t}}{\rho_{m} a_{m}} \int \frac{U}{U_{m}}\left(1-\left(\frac{U}{U_{m}}\right)^{2}\right) d Y=\left(\frac{T_{t}}{T_{m}}\right)^{\frac{\gamma+1}{2(\gamma-1)}} \delta_{3, t r}
$$


This means that a variable temperature profile has little effect on $H^{*}$ as it scales the momentum and kinetic energy thicknesses equally.

$$
H_{32}=\frac{\delta_{3}}{\delta_{2}}=\frac{\delta_{3, t r}}{\delta_{2, t r}}=\frac{\delta_{3, i}}{\delta_{2, i}}=H_{32, i}
$$

Now investigating displacement thickness, $\delta_{1}$ :

$$
\delta_{1}=\int\left(1-\frac{\rho u}{\rho_{m} u_{m}}\right) d y=\int \frac{\rho}{\rho_{m}}\left(\frac{\rho_{m}}{\rho}-\frac{u}{u_{m}}\right) d y
$$

Transforming coordinate system:

$$
\delta_{1}=\int\left(1-\frac{\rho u}{\rho_{m} u_{m}}\right) d y=\frac{\rho_{t} a_{t}}{\rho_{m} a_{m}} \int\left(\frac{T}{T_{m}}-\frac{U}{U_{m}}\right) d Y
$$

To make progress, the temperature distribution will be related to the velocity distribution. The static temperature distribution through the boundary layer may be expressed:

$$
\begin{aligned}
\frac{T}{T_{m}} & =\frac{T_{t}}{T_{m}}\left[\frac{T_{w}}{T_{t}}-\frac{u_{m}^{2}}{2 c_{p} T_{t}}\left(\frac{u}{u_{m}}\right)^{2}\right] \\
& =\frac{T_{t}}{T_{m}}\left[\frac{T_{w}}{T_{t}}-\left(1-\frac{T_{m}}{T_{t}}\right)\left(\frac{U}{U_{m}}\right)^{2}\right] \\
& =\frac{T_{t}}{T_{m}} \frac{T_{w}}{T_{t}}-\left(\frac{T_{t}}{T_{m}}-1\right)\left(\frac{U}{U_{m}}\right)^{2}
\end{aligned}
$$

Subtracting the transformed velocity profile from Equation C.68:

$$
\frac{T}{T_{m}}-\frac{U}{U_{m}}=\frac{T_{t}}{T_{m}}\left(\frac{T_{w}}{T_{t}}-\frac{U}{U_{m}}\right)+\left(\frac{T_{t}}{T_{m}}-1\right)\left(\frac{U}{U_{m}}\right)\left(1-\frac{U}{U_{m}}\right)
$$

Substituting Equation C.69 into C.67:

$$
\delta_{1}=\left(\frac{T_{t}}{T_{m}}\right)^{3}\left[\frac{T_{t}}{T_{m}} \delta_{1, t r}+\left(\frac{T_{t}}{T_{m}}-1\right) \delta_{2, t r}\right]
$$

Dividing by $\delta_{2}$ and applying Equation C.62: 


$$
H_{12}=\frac{T_{t}}{T_{m}} \underbrace{\left(\frac{\delta_{1, t r}}{\delta_{2, t r}}\right)}_{H_{12, t r}}+\left(\frac{T_{t}}{T_{m}}-1\right)
$$

This relates the compressible shape factor to the dummy transformed coordinate system. Now the link between the dummy system and an incompressible system is sought. Consider the Van Driest temperature distribution:

$$
\frac{T_{t}(y)}{T_{t, m}}=\underbrace{\frac{T_{w}}{T_{t, m}}}_{a}+\underbrace{\left(\frac{T_{a w}}{T_{t, m}}-\frac{T_{w}}{T_{t, m}}\right)}_{b} \frac{u}{u_{m}}+\underbrace{\left(1-\frac{T_{a w}}{T_{t, m}}\right)}_{c}\left(\frac{u}{u_{m}}\right)^{2}=a+b \frac{U}{U_{m}}+c\left(\frac{U}{U_{m}}\right)^{2}
$$

Applying this to the transformed displacement thickness:

$$
\delta_{1, t r}=\int\left[a\left(1-\frac{U}{U_{m}}\right)-c \frac{U}{U_{m}}\left(1-\frac{U}{U_{m}}\right)\right] d Y=a \delta_{1, i}-c \delta_{2, i}
$$

Dividing by $\delta_{2, i}$ leads to:

$$
H_{12, t r}=a H_{12, i}-c
$$

Substituting Equation C.74 into C.71:

$$
H_{12}=\frac{T_{w}}{T_{m}} H_{12, i}+\frac{T_{a w}}{T_{m}}-1
$$

The density flux thickness, $\delta_{\rho}$, can be shown to equal the enthalpy thickness, $\delta_{H}$, for flat plate boundary layers:

$$
\delta_{\rho}=\int \frac{u}{u_{m}}\left(1-\frac{\rho}{\rho_{m}}\right) d y=\underbrace{\int \frac{u \rho}{u_{m} \rho_{m}}\left(\frac{h}{h_{m}}-1\right) d y}_{\delta_{H}}=\frac{\rho_{0} a_{0}}{\rho_{m} a_{m}} \int \frac{U}{U_{m}}\left(\frac{T}{T_{m}}-1\right) d Y
$$

Using Equation C.68:

$$
\frac{U}{U_{m}}\left(\frac{T}{T_{m}}-1\right)=\frac{T_{t}}{T_{m}} \frac{U}{U_{m}}\left(\frac{T_{w}}{T_{t, m}}-1\right)+\left(\frac{T_{t}}{T_{m}}-1\right)\left(\frac{U}{U_{m}}\right)\left(1-\left(\frac{U}{U_{m}}\right)^{2}\right)
$$

Substituting Equation C.77 into C.76: 


$$
\delta_{\rho}=\left(\frac{T_{t, m}}{T_{m}}\right)^{3}\left[\frac{T_{t}}{T_{m}} \delta_{\rho, t r}+\left(\frac{T_{t}}{T_{m}}-1\right) \delta_{3, t r}\right]
$$

Dividing by $\delta_{2}$ and applying Equations C.62 and C.64:

$$
H_{\rho 2}=\frac{T_{t}}{T_{m}} \underbrace{\left(\frac{\delta_{\rho, t r}}{\delta_{2, t r}}\right)}_{H_{\rho 2, t r}}+\left(\frac{T_{t}}{T_{m}}-1\right) H_{32}
$$

Again, the aim is to relate the transformed coordinate system to the incompressible. Consider $\delta_{\rho, t r}$ and apply Equation C.72:

$$
\begin{aligned}
\delta_{\rho, t r} & =\int\left[\frac{U}{U_{m}}\left(\frac{T_{w}}{T_{t}}-1\right)\right] d Y \\
& =\int\left[(a-c-1) \frac{U}{U_{m}}\left(1-\frac{U}{U_{m}}\right)-c\left(\frac{U}{U_{m}}\right)\left(1-\left(\frac{U}{U_{m}}\right)^{2}\right)\right] d Y \\
& =-b \delta_{2, i}-c \delta_{3, i}
\end{aligned}
$$

Dividing by $\delta_{2, i}$ leads to:

$$
H_{\rho 2, t r}=-b-c H_{32, i}
$$

Substituting Equation C.81 into C.79:

$$
H_{\rho 2}=\left(\frac{T_{w}}{T_{m}}-\frac{T_{a w}}{T_{m}}\right)+\left(\frac{T_{a w}}{T_{m}}-1\right) H_{32, i}
$$

By assuming the Van Driest temperature distribution holds, the compressible shape factors $H_{\rho 2}, H_{32}$ and $H_{12}$ have been related to incompressible shape factors.

Now consider the kinetic energy integral equation. The form presented in Equation C.83 can be obtained by subtracting the Von Karman momentum equation.

$$
c_{d}=\frac{c_{f}}{2} \frac{H_{32}}{2}-\left(H_{\rho 2}-\frac{H_{32}}{2}\left(H_{12}-1\right)\right) \underbrace{\frac{\delta_{2}}{u_{m}} \frac{\partial u_{m}}{\partial x}}_{\Lambda}+\frac{\delta_{2}}{2} \frac{\partial H_{32}}{\partial x}
$$

Substituting Equations C.82, C.65 and C.75 for $H_{\rho 2}, H_{32}$ and $H_{12}$ respectively into Equation C.83: 


$$
c_{d}=\underbrace{\frac{c_{f}}{2} \frac{H_{32, i}}{2}}_{\text {Flat plate }}-\underbrace{\left(\left(\frac{T_{w}}{T_{m}}-\frac{T_{a w}}{T_{m}}\right)-\frac{H_{32, i}}{2}\left(\frac{T_{w}}{T_{m}} H_{12, i}-\frac{T_{a w}}{T_{m}}\right)\right)}_{\text {Pressure-gradient coupling }} \Lambda+\underbrace{\frac{\delta_{2}}{2} \frac{\partial H_{32, i}}{\partial x}}_{\approx 0}
$$

Laminar low-speed flow, $H_{32, i} \approx 1.57$ and $H_{12, i} \approx 2.59$ which leads to the pressuregradient coupling becoming:

$$
\frac{T_{w}}{T_{m}}\left(\frac{H_{32, i}}{2} H_{12, i}-1\right)+\frac{T_{a w}}{T_{m}}\left(1-\frac{H_{32, i}}{2}\right) \approx \frac{T_{w}}{T_{m}}+0.215
$$

Turbulent low-speed flow, $H_{32, i} \approx 1.75$ and $H_{12, i} \approx 1.4$ which leads to the pressuregradient coupling becoming:

$$
\frac{T_{w}}{T_{m}}\left(\frac{H_{32, i}}{2} H_{12, i}-1\right)+\frac{T_{a w}}{T_{m}}\left(1-\frac{H_{32, i}}{2}\right) \approx 0.225 \frac{T_{w}}{T_{m}}+0.125
$$

\section{C.2 Compressible Blasius dissipation coefficient}

The compressible Blasius equations can be derived by transforming the coordinate system. This derivation stems from the analysis presented by Anderson (1989).

$$
\zeta=\mu_{m}^{2} \mathrm{R}_{X}, \quad \eta=Y \frac{u_{m}}{v_{m}} \frac{1}{\sqrt{2 C_{w} \mathrm{R}_{X}}}, \quad f^{\prime}=\frac{1}{\sqrt{C_{w}}} \frac{u}{u_{m}}, \quad g=\frac{h}{h_{m}}=\frac{T}{T_{m}}
$$

where:

$$
C_{w}=\frac{\mu_{w}}{\mu_{m}} \frac{T_{m}}{T_{w}}, \quad x=X, \quad \frac{d Y}{d y}=\frac{\rho}{\rho_{m}}
$$

The Chapman-Rubesin parameter, $C_{w}$, and acts to decouple the momentum and energy equations. This leads to the following Blasius equations:

$$
\begin{aligned}
& f^{\prime \prime \prime}+f f^{\prime \prime}=\beta\left(f^{2}-g\right) \\
& g^{\prime \prime}+\mathrm{P} f g^{\prime}=-\mathrm{P}(\gamma-1) \mathrm{M}_{m}^{2} f^{\prime 2}
\end{aligned}
$$

Consider the velocity gradient:

$$
\frac{\partial u}{\partial y}=\frac{\partial u}{\partial Y} \frac{\rho}{\rho_{m}}=\frac{\partial u}{\partial \eta} \frac{\partial \eta}{\partial Y} \frac{T_{m}}{T}=u_{m} \sqrt{C_{w}} f^{\prime \prime} \frac{u_{m}}{v_{m}} \frac{1}{\sqrt{2 C_{w} \mathrm{R}_{X}}} g^{-1}=u_{m} \frac{u_{m}}{v_{m}} \frac{1}{\sqrt{2 \mathrm{R}_{X}}} \frac{f^{\prime \prime}}{g}
$$


This velocity gradient can be used to find the viscous volumetric entropy production:

$$
\begin{aligned}
s_{v i s c}^{\prime \prime \prime} & \approx \frac{\mu}{T}\left(\frac{\partial u}{\partial y}\right)^{2} \\
& =\left[\frac{\mu_{m} C_{w}}{T_{m}} T\right]\left(\frac{1}{T}\right)\left[u_{m}^{2}\left(\frac{u_{m}}{v_{m}}\right)^{2}\left(\frac{1}{2 \mathrm{R}_{x}}\right)\left(\frac{f^{\prime \prime}}{g}\right)^{2}\right] \\
& =\rho_{m} u_{m}^{3} \frac{1}{T_{m}} \frac{u_{m}}{v_{m}} \frac{C_{w}}{2 \mathrm{R}_{x}}\left(\frac{f^{\prime \prime}}{g}\right)^{2}
\end{aligned}
$$

The transformed coordinate system becomes:

$$
d y=g \frac{v_{m}}{u_{m}} \sqrt{2 C_{w} \mathrm{R}_{x}} d \eta
$$

This allows integral properties to be determined. Consider the Reynolds number based upon momentum thickness:

$$
\begin{aligned}
\mathrm{R}_{\delta_{2}}=\frac{u_{m} \delta_{2}}{v_{m}} & =\frac{u_{m}}{v_{m}} \int_{0}^{\infty} \frac{\rho u}{\rho_{m} u_{m}}\left(1-\frac{u}{u_{m}}\right) d y \\
& =\sqrt{2 C_{w} \mathrm{R}_{x}} \underbrace{\int_{0}^{\infty} \sqrt{C_{w}} f^{\prime}(\eta)\left(1-\sqrt{C_{w}} f^{\prime}(\eta)\right) d \eta}_{\approx 0.4695 c_{w}^{\frac{1}{4}}} \\
& \approx 0.4695 \sqrt{2 \mathrm{R}_{x}} C_{w}^{\frac{3}{4}}
\end{aligned}
$$

The skin friction becomes:

$$
\begin{aligned}
c_{f} & =\frac{\mu_{w} \frac{\partial u}{\partial y_{w}}}{0.5 \rho_{m} u_{m}^{2}} \\
& =\frac{\mu_{w} u_{m}}{\rho_{m} u_{m}^{2}} \frac{u_{m}}{v_{m}} \frac{2}{\sqrt{2 \mathrm{R}_{x}}} \frac{f^{\prime \prime}(0)}{g(0)} \\
& =\frac{\mu_{w} T_{m}}{\mu_{m} T_{w}} \frac{2}{\sqrt{2 \mathrm{R}_{x}}} \underbrace{f^{\prime \prime}(0)}_{0.4695 c_{w}^{-\frac{3}{4}}} \\
& =0.44 \frac{C_{w}}{\mathrm{R}_{\delta_{2}}}
\end{aligned}
$$

The dissipation coefficient becomes: 


$$
\begin{aligned}
c_{d} & =\frac{\int T s_{v i s c}^{\prime \prime \prime} d y}{\rho_{m} u_{m}^{3}} \\
& =\int g^{2} C_{w} \sqrt{\frac{C_{w}}{2 \mathrm{R}_{x}}\left(\frac{f^{\prime \prime}}{g}\right)^{2} d \eta} \\
& =\frac{C_{w} C_{w}^{\frac{5}{4}}}{\sqrt{2 \mathrm{R}_{x}} C_{w}^{\frac{3}{4}}} \underbrace{\int_{0}^{\infty} f^{\prime \prime 2} d \eta} \\
& \approx 0.173 \frac{C_{w}}{\mathrm{R}_{\delta_{2}}}
\end{aligned}
$$

Comparing the dissipation coefficient to skin friction, the relationship presented in the previous section is recovered.

$$
c_{d}=0.173 \frac{C_{w}}{\mathrm{R}_{\delta_{2}}}=\left(\frac{0.44}{2} \frac{C_{w}}{\mathrm{R}_{\delta_{2}}}\right)\left(\frac{1.57}{2}\right)=\frac{c_{f}}{2} \frac{H^{*}}{2}
$$

\section{C.3 Turbulent boundary layer profiles}

This semi-local scaling method described by Trettel \& Larsson (2016) has been applied here to write the dissipation coefficient in inner units. For boundary layers, the semi-local method is:

$$
\begin{aligned}
& Y^{+}=\frac{y}{l}=\frac{\bar{\rho}\left(\frac{\tau_{w}}{\bar{\rho}}\right)^{\frac{1}{2}} y}{\bar{\mu}} \\
& U^{+}=\int_{0}^{u^{+}}\left(\frac{\bar{\rho}}{\rho_{w}}\right)^{\frac{1}{2}}\left[1+\frac{1}{2} \frac{1}{\bar{\rho}} \frac{d \bar{\rho}}{d y} y-\frac{1}{\bar{\mu}} \frac{d \bar{\mu}}{d y} y\right] d u^{+} \\
& R_{u v}^{+}=\frac{\bar{\rho} r_{u v}}{\tau_{w}} \\
& \mathrm{R}_{\tau}^{*}=\frac{\rho_{m}\left(\frac{\tau_{w}}{\rho_{m}}\right)^{\frac{1}{2}} \delta}{\mu_{m}} \\
& u_{\tau}=\sqrt{\frac{\tau_{w}}{\rho_{w}}}, \quad u^{+}=\frac{u}{u_{\tau}}
\end{aligned}
$$

The total dissipation through a boundary layer is: 


$$
\int(\phi+\pi) d y=\int\left(\mu\left(\frac{d u}{d y}\right)^{2}-\overline{\rho u^{\prime \prime} v^{\prime \prime}} \frac{d u}{d y}\right) d y
$$

The aim is to rewrite Equation C.98 in inner units. First, consider the velocity gradient:

$$
\frac{d u}{d y}=\frac{d u}{d U} \frac{d Y}{d y} \frac{d U}{d Y}=\frac{d u}{d U} \frac{d Y}{d y}\left(\frac{d u}{d u^{+}} \frac{d y^{+}}{d y} \frac{d U^{+}}{d Y^{+}}\right)
$$

where:

$$
\begin{aligned}
& \frac{d U}{d u}=\left(\frac{\bar{\rho}}{\rho_{w}}\right)^{\frac{1}{2}}\left[1+\frac{1}{2} \frac{1}{\bar{\rho}} \frac{d \bar{\rho}}{d y} y-\frac{1}{\bar{\mu}} \frac{d \bar{\mu}}{d y} y\right] \\
& \frac{d Y}{d y}=\left(\frac{\mu_{w}}{\bar{\mu}}\right)\left(\frac{\bar{\rho}}{\rho_{w}}\right)^{\frac{1}{2}}\left[1+\frac{1}{2} \frac{1}{\bar{\rho}} \frac{d \bar{\rho}}{d y} y-\frac{1}{\bar{\mu}} \frac{d \bar{\mu}}{d y} y\right] \\
& \frac{d u^{+}}{d u}=\frac{1}{u_{\tau}} \\
& \frac{d y^{+}}{d y}=\left(\frac{\rho_{w} u_{\tau}}{\mu_{w}}\right)
\end{aligned}
$$

Therefore:

$$
\frac{d u}{d y}=\left(\frac{\rho_{w} u_{\tau}^{2}}{\bar{\mu}}\right) \frac{d U^{+}}{d Y^{+}}
$$

Second, consider $\overline{\rho u^{\prime \prime} v^{\prime \prime}}$ :

$$
\overline{\rho u^{\prime \prime} v^{\prime \prime}}=\bar{\rho} r_{u v}=\tau_{w} R_{u v}^{+}=\rho_{w} u_{\tau}^{2} R_{v y}^{+}
$$

Finally, consider the change in integration coordinate:

$$
y=Y^{+} l=Y^{+} \frac{\bar{\mu}}{\sqrt{\bar{\rho} \tau_{w}}}
$$

Differentiating: 


$$
\begin{aligned}
d y & =\left(\frac{d Y^{+}}{Y^{+}}+\frac{d l}{l}\right) l Y^{+} \\
& =\frac{\left(\frac{d Y^{+}}{Y^{+}}+\frac{d l}{l}\right)}{d Y^{+} / Y^{+}} l d Y^{+} \\
& =\underbrace{\left[1+\frac{1}{\bar{\mu}} \frac{d \bar{\mu}}{d Y^{+}} Y^{+}-\frac{1}{2} \frac{1}{\rho} \frac{d \rho}{d Y^{+}} Y^{+}\right]}_{\text {Change in length scale due to compressibility }} l d Y^{+} \\
& =\left(\frac{\rho_{w}}{\bar{\rho}}\right)^{\frac{1}{2}} \frac{\bar{\mu}}{\mu_{w}}\left[1+\frac{1}{\bar{\mu}} \frac{d \bar{\mu}}{d Y^{+}} Y^{+}-\frac{1}{2} \frac{1}{\rho} \frac{d \rho}{d Y^{+}} Y^{+}\right] l_{w} d Y^{+}
\end{aligned}
$$

Combining Equations C.98, C.101, C.102 and C.104 leads to:

$$
\begin{aligned}
\int(\phi+\pi) d y & =\int\left(\left(\frac{\mu_{w}}{\bar{\mu}}\right)\left(\frac{\rho_{w}^{2} u_{\tau}^{4}}{\mu_{w}}\right)\left(\frac{d U^{+}}{d Y^{+}}\right)^{2}-\left(\frac{\mu_{w}}{\bar{\mu}}\right)\left(\frac{\rho_{w}^{2} u_{\tau}^{4}}{\mu_{w}}\right) R_{v y}^{+} \frac{d U^{+}}{d Y^{+}}\right) d y \\
& =\left(\frac{\rho_{w}^{2} u_{\tau}^{4}}{\mu_{w}}\right) \int\left(\frac{\mu_{w}}{\bar{\mu}}\right)\left(\left(\frac{d U^{+}}{d Y^{+}}\right)^{2}-R_{v y}^{+} \frac{d U^{+}}{d Y^{+}}\right) d y \\
& =\rho_{w} u_{\tau}^{3} \int\left(\left(\frac{d U^{+}}{d Y^{+}}\right)^{2}-R_{v y}^{+} \frac{d U^{+}}{d Y^{+}}\right)\left(\frac{\rho_{w}}{\bar{\rho}}\right)^{\frac{1}{2}}\left[1+\frac{1}{\bar{\mu}} \frac{d \bar{\mu}}{d Y^{+}} Y^{+}\right. \\
& \left.-\frac{1}{2} \frac{1}{\rho} \frac{d \rho}{d Y^{+}} Y^{+}\right] d Y^{+}
\end{aligned}
$$







\section{Appendix D Design implications}

\section{D.1 Steady one-dimensional flow euergy}

Consider a single stream of perfect gas, the flow euergy is expressed by Equation D.106.

$$
\mathbf{m}_{\mathbf{f}}=h\left(1-\left(\frac{p_{0}}{p}\right)^{\frac{\gamma-1}{\gamma}}\right)+\frac{1}{2} V^{2}=h_{t}\left(1-\left(\frac{p_{0}}{p_{t}}\right)^{\frac{\gamma-1}{\gamma}}\right)
$$

Assuming the reference environment pressure, $p_{0}$, and the ratio of specific heats, $\gamma$, are constant, then:

$$
d\left[\left(\frac{p_{0}}{p_{t}}\right)^{\frac{\gamma-1}{\gamma}}\right]=-\frac{\gamma-1}{\gamma}\left(\frac{p_{0}}{p_{t}}\right)^{\frac{\gamma-1}{\gamma}} \frac{d p_{t}}{p_{t}}
$$

Differentiating Equation D.106 and substituting Equation D.107:

$$
d \mathbf{m}_{\mathbf{f}}=\left(1-\left(\frac{p_{0}}{p_{t}}\right)^{\frac{\gamma-1}{\gamma}}\right) d h_{t}+h_{t} \frac{\gamma-1}{\gamma}\left(\frac{p_{0}}{p_{t}}\right)^{\frac{\gamma-1}{\gamma}} \frac{d p_{t}}{p_{t}}
$$

Dividing Equation D.108 by the flow euergy illustrates that the fractional change in flow euergy is due to fractional changes in total temperature and total pressure:

$$
\frac{d \mathbf{m}_{\mathbf{f}}}{\mathbf{m}_{\mathbf{f}}}=\frac{d T_{t}}{T_{t}}+\underbrace{\frac{\gamma-1}{\gamma} \frac{\left(\frac{p_{0}}{p_{t}}\right)^{\frac{\gamma-1}{\gamma}}}{1-\left(\frac{p_{0}}{p_{t}}\right)^{\frac{\gamma-1}{\gamma}}} \frac{d p_{t}}{p_{t}}}_{A}
$$


where $A$ will be used in this section to simplify the algebra.

The change in total pressure is due to changes in total temperature and friction. For onedimensional flow, the result obtained by Shapiro can be applied:

$$
\frac{d p_{t}}{p_{t}}=-\frac{\gamma \mathrm{M}^{2}}{2}\left[\frac{d T_{t}}{T_{t}}+4 f \frac{d x}{D}\right]
$$

Substituting Equations D.110 into D.109:

$$
\frac{d \mathbf{m}_{\mathbf{f}}}{\mathbf{m}_{\mathbf{f}}}=\underbrace{\left(1-A \frac{\gamma \mathrm{M}^{2}}{2}\right) \frac{d T_{t}}{T_{t}}}_{\text {Due to heat transfer }}-\underbrace{A \frac{\gamma \mathrm{M}^{2}}{2}\left(4 f \frac{d x}{D}\right)}_{\text {Due to friction }}
$$

This indicates the fraction change in flow euergy is due to heat transfer and viscous dissipation. However, for forced convection these two mechanisms do not act independently. Considering the heat leaving a stream:

$$
d Q=h_{t} \frac{d T_{t}}{T_{t}}=h_{t}\left(\frac{T_{w}}{T_{t}}-\frac{T_{w a}}{T_{t}}\right) 2 f \frac{d x}{D}
$$

Equation D.112 demonstrates that changes in total temperature do not occur independently of friction in forced convection.

Now, consider two streams of perfect gas with heat being transferred between them. By conservation of energy and using Equation D.112:

$$
\begin{aligned}
\dot{m}_{m} d Q_{m} & =-\dot{m}_{c} d Q_{c} \\
\dot{m}_{m} h_{t, m}\left[\left(\frac{T_{w}}{T_{t}}-\frac{T_{w a}}{T_{t}}\right) 2 f \frac{d x}{D}\right]_{m} & =-\dot{m}_{c} h_{t, c}\left[\left(\frac{T_{w}}{T_{t}}-\frac{T_{w a}}{T_{t}}\right) 2 f \frac{d x}{D}\right]_{c}
\end{aligned}
$$

where the subscript $m$ represents the mainstream and the subscript $c$ represents the coolant.

Equation D.113 demonstrates how the friction of the two streams can be related based upon the heat transferred between them. 
The total change in the ability of a turbine to extract work from the system is shown in Equation D.114.

$$
\begin{aligned}
d \mathbf{M}_{\mathbf{f}}= & \dot{m}_{m} \mathbf{m}_{\mathbf{f}, m} \frac{d \mathbf{m}_{\mathbf{f}, m}}{\mathbf{m}_{\mathbf{f}, m}}+\dot{m}_{c} \mathbf{m}_{\mathbf{f}, c} \frac{d \mathbf{m}_{\mathbf{f}, c}}{\mathbf{m}_{\mathbf{f}, c}} \\
= & \underbrace{\left\{\dot{m}_{m} \mathbf{m}_{\mathbf{f}, m}\left[\frac{d T_{t}}{T_{t}}\left(1-\frac{A \gamma \mathbf{M}^{2}}{2}\right)\right]_{m}+\dot{m}_{c} \mathbf{m}_{\mathbf{f}, c}\left[\frac{d T_{t}}{T_{t}}\left(1-\frac{A \gamma \mathrm{M}^{2}}{2}\right)\right]_{c}\right\}}_{\text {Thermal creation, } \Phi_{t h}^{m}} \\
& -\underbrace{\left\{\dot{m}_{m} \mathbf{m}_{\mathbf{f}, m}\left[2 A \gamma M^{2} f \frac{d x}{D}\right]_{m}+\dot{m}_{c} \mathbf{m}_{\mathbf{f}, c}\left[2 A \gamma M^{2} f \frac{d x}{D}\right]_{c}\right\}}_{\text {Viscous dissipation, } \Phi_{v}^{\mathbf{m}}}
\end{aligned}
$$

The work that could be extracted is altered by thermal creation, due to heat transfer between the two streams, and viscous dissipation, due to friction occurring within each stream. As shown in Equations D.112 and D.113, heat transfer and friction are not independent for forced convection. This means the process of thermal creation is not independent from viscous dissipation.

The change in the system is governed by the ratio of the effect of thermal creation to the effect of viscous dissipation.

$$
d \mathbf{M}_{\mathbf{f}}=\Phi_{t h}^{\mathbf{m}}-\Phi_{v}^{\mathbf{m}}=\Phi_{v}^{\mathbf{m}}\left(\frac{\Phi_{t h}^{\mathbf{m}}}{\Phi_{v}^{\mathbf{m}}}-1\right)
$$

Examining the effect of thermal creation on this system:

$$
\begin{aligned}
\Phi_{t h}^{\mathbf{m}} & =\dot{m}_{m} \mathbf{m}_{\mathbf{f}, m} \frac{d T_{t, m}}{T_{t, m}}\left(1-\frac{A \gamma \mathrm{M}^{2}}{2}\right)_{m}-\dot{m}_{m} \mathbf{m}_{\mathbf{f}, m} \frac{d T_{t, m}}{T_{t, m}} \frac{\mathbf{m}_{\mathbf{f}, c}}{\mathbf{m}_{\mathbf{f}, m}} \frac{h_{t, m}}{h_{t, c}}\left(1-\frac{A \gamma \mathrm{M}^{2}}{2}\right)_{c} \\
& =\dot{m}_{m} \mathbf{m}_{\mathbf{f}, m} \frac{d T_{t, m}}{T_{t, m}}\left\{\left(1-\frac{A \gamma \mathrm{M}^{2}}{2}\right)_{m}-\frac{\mathbf{m}_{\mathbf{f}, c}}{\mathbf{m}_{\mathbf{f}, m}} \frac{h_{t, m}}{h_{t, c}}\left(1-\frac{A \gamma \mathrm{M}^{2}}{2}\right)_{c}\right\}_{m}\left[1-\frac{\left(\frac{p_{0}}{p_{t, c}}\right)^{\frac{\gamma-1}{\gamma}}}{\left(1-\frac{A \gamma \mathrm{M}^{2}}{2}\right)_{c}} \frac{p_{0}}{p_{t, m}}\right)^{\frac{\gamma-1}{\gamma}}\left(1-\frac{A \gamma \mathrm{M}^{2}}{2}\right)_{m}
\end{aligned}
$$


Examining the effect of viscous dissipation on this system:

$$
\Phi_{v}^{\mathbf{m}}=\dot{m}_{m} \mathbf{m}_{\mathbf{f}, m}\left[A \gamma \mathrm{M}^{2} 2 f \frac{d x}{D}\right]_{m}\left\{1-\frac{\left(A \gamma \mathrm{M}^{2}\right)_{c}}{\left(A \gamma \mathrm{M}^{2}\right)_{m}} \frac{1-\left(\frac{p_{0}}{p_{t, c}}\right)^{\frac{\gamma-1}{\gamma}}}{1-\left(\frac{p_{0}}{p_{t, m}}\right)^{\frac{\gamma-1}{\gamma}}} \frac{\left(\frac{T_{w}}{T_{t}}-\frac{T_{w a}}{T_{t}}\right)_{m}}{\left(\frac{T_{w}}{T_{t}}-\frac{T_{w a}}{T_{t}}\right)_{c}}\right\}
$$

The ratio of Equations D.116 and D.117 indicate the relative magnitude of the effect of thermal creation to the effect of viscous dissipation. This ratio must be greater than unity for thermal creation to overcome viscous dissipation.

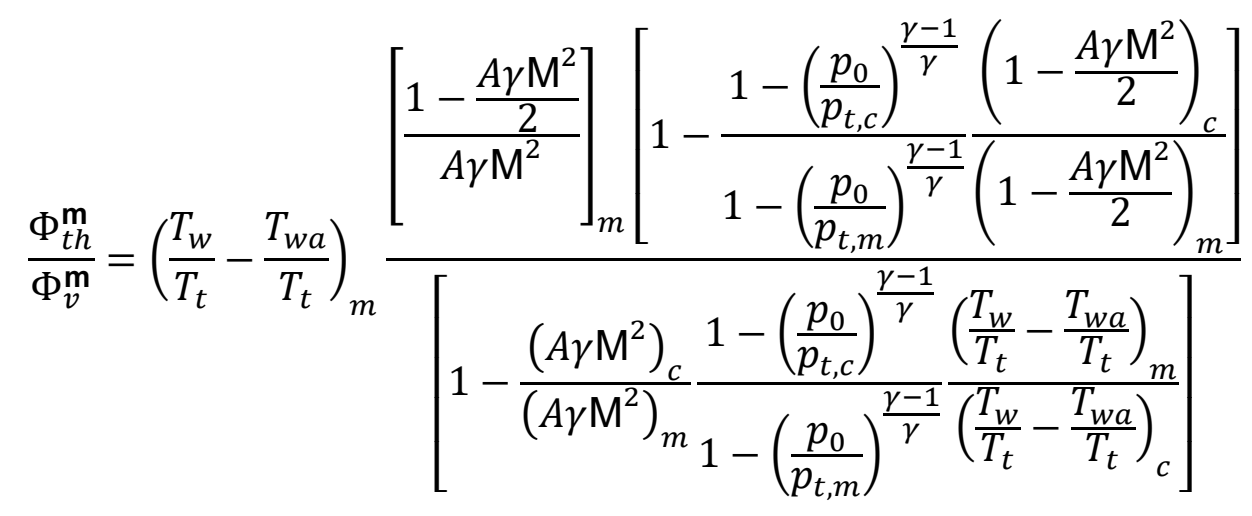

Equation D.118 can be simplified assuming the total pressure and ratio of specific heats are the same for the two streams:

$$
\frac{\Phi_{t h}^{\mathrm{m}}}{\Phi_{v}^{\mathrm{m}}}=\frac{-\frac{1}{2}\left[1-\frac{\mathrm{M}_{c}^{2}}{\mathrm{M}_{m}^{2}}\right]\left(\frac{T_{w}}{T_{t}}-\frac{T_{w a}}{T_{t}}\right)_{m}}{1-\frac{\mathrm{M}_{c}^{2}}{\mathrm{M}_{m}^{2}} \frac{\left(\frac{T_{w}}{T_{t}}-\frac{T_{w a}}{T_{t}}\right)_{m}}{\left(\frac{T_{w}}{T_{t}}-\frac{T_{w a}}{T_{t}}\right)_{c}}}
$$


